All inclusive?!

Aspekte einer inklusiven Musik- und Tanzpädagogik Heike Henning (Hrsg.) 


\section{Innsbrucker Perspektiven zur Musikpädagogik}

herausgegeben vom

Department für Musikpädagogik Innsbruck der Universität Mozarteum Salzburg

Band 2 
Heike Henning (Hrsg.)

All inclusive?!

Aspekte einer inklusiven

Musik- und Tanzpädagogik

Waxmann 2020

Münster · New York 
Mit freundlicher Unterstützung der Abt.

Landesmusikdirektion des Landes Tirol.

Bibliografische Informationen der Deutschen Nationalbibliothek

Die Deutsche Nationalbibliothek verzeichnet diese Publikation in der Deutschen Nationalbibliografie; detaillierte bibliografische

Daten sind im Internet über http://dnb.dnb.de abrufbar.

\section{Innsbrucker Perspektiven zur Musikpädagogik, Band 2}

ISSN 2626-6547

Print-ISBN 978-3-8309-4276-4

E-Book-ISBN 978-3-8309-9276-9 (Open Access)

https://doi.org/10.31244/9783830992769

(c) Waxmann Verlag GmbH, 2020

Steinfurter Straße 555, 48159 Münster

www.waxmann.com

info@waxmann.com

Umschlaggestaltung: Christian Mariacher, Informationsdesign, Innsbruck Satz: Stoddart Satz- und Layoutservice, Münster

Dieses Buch ist verfügbar unter folgender Lizenz: CC-BY-NC-ND 4.0 Namensnennung-Nicht kommerziell-Keine Bearbeitungen 4.0 International

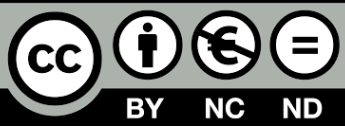




\section{Inhalt}

Kunst und Kultur für alle - All inclusive?!

Ein Vorwort von Martina Fladerer und Heike Henning

All inclusive?! Theoretische Zugänge zur Inklusion

Michael Turinsky

Crip Choreography

Lisa Pfahl

Zur gesellschaftlichen Inklusion kulturell-künstlerischer

Repräsentationen von Behinderung

Persson Perry Baumgartinger \& Anita Moser

Frictions and Fractions?!

Kritische Perspektiven auf Kulturarbeit, Kulturvermittlung und Diversity 25

Renate Reitinger

Chancengleichheit als Entwicklungsaufgabe an Musikhochschulen

Bedingungen - Strukturen - Möglichkeiten

Podiumsdiskussion

Die Qualität des Nicht-Synchron-Seins - Gedanken zur Funktionalität und Widerspenstigkeit.

\section{Inklusion in musik- oder tanzpädagogischen Kontexten}

Erik Esterbauer

Einschätzung von Beziehungsqualität als Grundpfeiler inklusiven Arbeitens

Das EBQ-Instrument in der Praxis.

Julia Lutz

Von der Erfahrung über die Reflexion zur Perspektivenübernahme Impulse zur Planung und Durchführung musikalischer Aktivitäten in heterogenen Gruppen

Julia Eibl

Ziele und Inhalte des Instrumentalunterrichts mit Menschen mit

Behinderung aus der Perspektive österreichischer Musikschullehrkräfte

Shirley Salmon

Die Bedeutung des Orff-Schulwerks für inklusiven Musikunterricht

Nora Schnabl-Andritsch, Virginie Roy \& Michelle Proyer

The Art of Inclusion: Modellierung polyästhetischer Ansätze in der Inklusion 
Mirjam Hoffmann

Capoeira als inklusive Praxis

Pädagogisches Potenzial einer brasilianischen Widerstandsbewegung

Evelyne Walser-Wohlfarter \& Bernhard Richarz

Zeitgenössisch in Vielfalt unterrichten

Aus der Praxis einer Tanzinitiative

Beate Hennenberg \& Hana Zanin

Bewusstseinswandel durch tanzkünstlerische Aktivitäten des inklusiv

arbeitenden Tanzvereins Ich bin O.K.

Stephanie Bangoura

Gemeinschaft tanzen - Annäherungen an

eine rituelle Tanzpädagogik mit Livemusik

Musik- und tanzpädagogische Praxen in inklusiven und musikgeragogischen Kontexten

Mona Heiler

KlaWIR am Bahnhof

Das gemeinschaftsbildende Potenzial eines Community-Music-

Projekts im öffentlichen Raum.

Stefan Greuter

POWERband Tirol: eine inklusive Band im Fokus

Bettina Büttner-Krammer

Young Symphony - ein inklusives Education-Programm

der Wiener Symphoniker

Don Quixote reloaded mit der Partnerschule Neue Mittelschule Gassergasse .....217

Regina Brandhuber

Alles ganz normal!?

Franziska Lottner erhält einen 1. Preis bei Jugend musiziert

Christine Schönherr

„Man spürt, dass man lebt“ Musik-Sprache-Bewegung/Tanz

Künstlerisch-geragogische Angebote für Menschen in hohem Alter

Marc Brand

„Musik ist mein Leben“

Gemeinsam Musiklernen im Seniorenalter - eine filmische Annäherung

Autorinnen und Autoren 


\section{Kunst und Kultur für alle - All inclusive?!}

\section{Ein Vorwort von Martina Fladerer und Heike Henning}

Was muss sich verändern, damit musik- und tanzpädagogische Angebote für alle Menschen Möglichkeiten bieten, sich künstlerisch-musikalisch zu entfalten und auszudrücken? Diese Frage stand im Zentrum des Symposiums All Inclusive?! - Internationales Symposium einer inklusiven Musik- und Tanzpädagogik, das vom 12. bis 13. Oktober 2018 im neu eröffneten Haus der Musik in Innsbruck stattfand. Als Kooperationsprojekt der drei führenden $\mathrm{Mu}$ sikausbildungsstätten Tirols, namentlich der Universität Mozarteum Salzburg, Department für Musikpädagogik Innsbruck, dem Tiroler Landeskonservatorium und dem Tiroler Landesmusikschulwerk, war die Tagung als eine bunte Veranstaltung konzipiert, die wissenschaftliche Beiträge und Praxisworkshops gleichermaßen beinhaltete. Bereits im Vorfeld zeigte sich durch die überwältigende Resonanz auf die Ausschreibung des Symposiums, wie aktuell Fragen nach Teilhabe und Chancengleichheit sind.

Inklusion verharrt nicht auf individueller Ebene. Als ein kontinuierlicher Weg hin zu mehr Vielfalt erfordert sie das Hinterfragen und möglicherweise die Veränderung institutioneller Strukturen, welche an der Konstitution von Ausschlusskriterien beteiligt sind. Es gilt Mechanismen, Machtstrukturen und Traditionen zu überwinden, welche die Teilhabe an Kunst und Kultur verhindern, da diese als staatliche Pflicht ${ }^{1}$ festgeschrieben wurde. Dies kann Reibungen verursachen, denn Inklusion als gelebte und positiv bewertete Vielfalt ist kein statischer Endzustand, sondern ein steter Prozess. Inklusion beinhaltet auch, dass sich Diversität auf allen gesellschaftlichen und institutionellen Ebenen deutlicher widerspiegelt. Erstens auf der Personalebene, auf der Strukturen so verändert werden sollten, dass bislang marginalisierte Gesellschaftsgruppen Mitbestimmungsrechte in höheren Hierarchieebenen erhalten; zweitens auf programmatischer Ebene. Hier ist zu fragen, ob und inwieweit sich die gesellschaftliche Vielfalt konkret im kulturellen Angebot zeigt. Zuletzt auf $\mathrm{Pu}$ blikumsebene: Wer nimmt Angebote wahr, wer bleibt aus welchen Gründen den Angeboten fern?

Ausschließende Mechanismen auf all diesen Ebenen offenzulegen ist notwendig, um die Teilhabe aller zu ermöglichen. Hierzu ist auch ein veränderter Blick nötig, der jeden künstlerischen Ausdruck wie eine eigene Kunstform mit

1 Laut UN-Behindertenrechtskonvention muss es allen Menschen ermöglicht werden, „ihr kreatives, künstlerisches und intellektuelles Potenzial zu entfalten und zu nutzen“" (Artikel 30 Absatz 2 der UN-Behindertenrechtskonvention). 
spezifischer Ästhetik betrachtet. Anstatt etablierte und tradierte Maßstäbe unhinterfragt zu übernehmen, gilt es, Formen des künstlerischen Ausdrucks zu de- und reorganisieren. Umgesetzt werden kann das beispielsweise, indem im Musikschulunterricht alle Schüler*innen als fähige Individuen, die nur bedingt vergleichbar in ihrer Entwicklung und ihren Ausdrucksweisen sind, wahrgenommen werden. Andersheit und Individualität können so künstlerisches Potenzial bedeuten. Eine solche musik- oder tanzpädagogische Grundhaltung ist ressourcenorientiert und fokussiert keine Defizite - was in der zeitgemäßen Pädagogik ohnehin als obsolet gilt. Ein lustvolles und kreatives Spiel zwischen Beat und Off-Beat, zwischen dem In-Takt-Sein und dem Aus-dem-Takt-Geraten entfaltet sich, in dem keine Stereotype reproduziert werden, sondern in dem sich neue Spielräume entdecken und kreieren lassen. Musikschulen könnten zu Orten werden, an denen Menschen unterschiedlicher sozialer Schichten in vorbildlicher Art und Weise vereint werden. Orte, die in besonderem Maße gemeinschaftsstiftend, interkulturell, generations- und kulturübergreifend sind und von denen eine solche Wirkung ausgeht.

Von dieser inklusiven Vision und Grundhaltung sowie von einem Ja zur Vielfalt geleitet, beschreiben die Autor ${ }^{*}$ inn)en in ihren Beiträgen theoretische und praxisorientierte, musik- bzw. tanzpädagogische Aspekte und -projekte dieses Inklusionsverständnisses.

Der Symposiumsband ist in drei Teile gegliedert. Im ersten Teil des Buches finden sich theoretische Zugänge zur Inklusion. So erläutert der Choreograph und Tänzer Michael Turinsky (Gewinner des Nestroy-Preises 2017) in seinem hier verschriftlichten Keynote-Vortrag, dass die zwanghafte Funktionstüchtigkeit des Körpers aus gesellschaftlichen Strukturen resultiert (u. a. aus dem Zeitknappheitsparadigma), die es zu hinterfragen gilt. Er gibt zu bedenken, dass Körper möglicherweise ein ganz eigenes, individuelles Verhältnis zu Zeit hätten. Was ist, wenn die in Musik und Tanz abverlangte Synchronizität für einzelne Individuen unmöglich ist? Hierfür skizziert er in seinem Beitrag Wege, diese fehlende Funktionstüchtigkeit künstlerisch-performativ zu nutzen. In den folgenden Beiträgen von Lisa Pfahl, Persson Perry Baumgartinger und Anita Moser wird die Repräsentation von Personen (mit und ohne Behinderungen), die der vermeintlichen Norm nicht entsprechen, in unterschiedlichen Bereichen und Ebenen der Gesellschaft beschrieben und kritisch hinterfragt. Renate Reitinger definiert Chancengleichheit als eine Entwicklungsaufgabe von Musikhochschulen und gibt Einblicke in das von der Hochschule für Musik Nürnberg entwickelte Konzept zur Chancengleichheit. Schließlich werden verschiedene Aspekte von Inklusion und möglichen exkludierenden Barrieren beleuchtet, welche auf der Podiumsdiskussion debattiert wurden. 
Im zweiten Teil des Buches finden sich musik- und tanzpädagogische Beiträge zu Inklusion. Erik Esterbauer, Julia Lutz und Julia Eibl betrachten in ihren Artikeln über das Gelingen von Inklusion wesentliche pädagogische Aspekte, wie Beziehungsqualität, Reflexion, Perspektivenübernahme oder die Sicht der Musikschullehrkräfte auf ihre inklusiven Unterrichtserfahrungen. Shirley Salmon zeigt die Bedeutung des Orff-Schulwerks für inklusiven Musikunterricht anhand eines Praxisbeispiels auf. Das Autorinnentrio Nora Schnabl-Andritsch, Virginie Roy und Michelle Proyer erläutert einen in der Praxis erprobten und wissenschaftlich begleiteten polyästhetischen Ansatz von Inklusion. Am Ende des zweiten Teiles stehen vier tanzpädagogische Beiträge: Mirjam Hoffmann beschreibt das inklusive Potenzial der Capoeira, einem brasilianischen Kampftanz. Evelyne Walser-Wohlfarter und Bernhard Richarz berichten von ihrer Initiative tanzfähig, bei der Zeitgenössischer Tanz allen zugänglich gemacht wird, die tanzen wollen, unabhängig von Alter, Behinderung, Herkunft und Vorerfahrung. Beate Hennenberg und Hana Zanin geben Einblicke in die Arbeit des Tanzvereins Ich bin O.K. aus Wien. Abschließend schildert Stephanie Bangoura die Bedeutung ritueller Elemente in der zeitgenössischen Tanzpädagogik und erläutert ihre, auf dieser Basis entwickelte, transkulturelle und inklusive Methode.

Im dritten Teil des Buches werden verschiedene konkrete musik- und tanzpädagogische Praxen in Diversitätskontexten vorgestellt. Diese Praxislichter bzw. Best-Practice-Beispiele zeigen, dass und in welcher Art und Weise erfolgreiche musik- oder tanzpädagogische Projekte Teilhabe ermöglichen. Mona Heiler beschreibt in KlaWIR am Bahnhof ein von ihr entwickeltes Community-MusicProjekt, das sie im Rahmen ihrer Dissertation wissenschaftlich begleitet. Stefan Greuter gibt Einblick in die Arbeit der POWERband TIROL, eine Band, welche ausschließlich aus Musiker( ${ }^{*}$ inne)n mit Beeinträchtigungen besteht. Regelmäßige Probenarbeit und öffentliche Auftritte zusammen mit Jazzmusiker( ${ }^{*}$ inne)n aus der Region Innsbruck unter der Leitung von Christoph Heiss kennzeichnen diese Band, welche beim Symposium live zu erleben war. Bettina BüttnerKrammer gibt Einblicke in das inklusiv gestaltete Education-Programm der Wiener Symphoniker. Dass die Teilnahme von Menschen mit Beeinträchtigung an konventionellen Musikwettbewerben wie bspw. Jugend musiziert noch immer die Ausnahme bildet, aber erfolgreich gelingen kann, legt Regina Brandhuber in ihrem Bericht dar. Am Ende des Tagungsbandes stehen zwei Beiträge von Christine Schönherr und Marc Brand, welche das inklusive Potenzial zweier musikgeragogischer Angebote beschreiben. 
All diese hier dargestellten Methoden und Praxislichter möchten Mut machen, verfestigte Strukturen und künstlerische Wertigkeiten zu hinterfragen, um mit Offenheit und Kreativität neue Formate ins Leben zu rufen.

An dieser Stelle gilt der besondere Dank den beiden Mitorganisatorinnen des Symposiums, Christine Knoll-Kaserer (Tiroler Landeskonservatorium) und Andrea Albrecht (Tiroler Musikschulwerk), für die gute Zusammenarbeit bei der Planung und Durchführung von All Inclusive?!

Ebenfalls danke ich, Heike Henning, meinen Studienassistentinnen Sara Dallinger und Andrea Kapeller für ihre intensive Mitarbeit bei der Herausgabe dieses Tagungsbandes sowie Milena Dmoch und Kristina Glücker für ihre Mithilfe beim Korrektorat. 


\section{Crip Choreography ${ }^{1}$}

\section{Zur Problematik des Begriffs Inklusion}

Es freut mich sehr, über Inklusion sprechen zu dürfen. Dieses Thema erscheint uns wichtig. Auch ich habe lange Jahre meine eigene künstlerische Praxis als inklusiven Tanz oder inklusive Choreografie bezeichnet. Nun muss ich allerdings gleich zu Beginn meines Vortrags bekennen, dass mich das Thema Inklusion mittlerweile nicht mehr so brennend interessiert. Ich selber bin in den letzten zwei bis drei Jahren dazu übergegangen, diesen von mir selbst verwendeten Begriff fallen zu lassen. Hierfür möchte ich drei Gründe nennen.

Der erste Grund betrifft meinen eigenen Standpunkt. Ich denke, es ist Ihnen allen bekannt, dass der Begriff Inklusion seinen Ursprung in einem pädagogischen Diskurs hat. Er ist im Prinzip ein pädagogischer Begriff. Vielleicht ist er auch ein politscher Begriff, darüber könnte man streiten. Trotzdem kommt er primär in pädagogischen Zusammenhängen vor. Ich aber war nie ein besonders guter Pädagoge und verstehe mich eher als jemand, der künstlerisch arbeitet und der deshalb auch aus der Position eines behinderten Künstlers spricht. Daher erschien mir der Begriff Inklusion für mich und meine Sichtweise nicht mehr sinnvoll.

Der zweite Grund betrifft gewissermaßen das Verständnis von Ein- und Ausschluss, das er suggeriert. Und ich sage bewusst suggeriert, weil ich nicht behaupten will, dass er ein bestimmtes Verständnis von Ein- oder Ausschluss ausdrücklich formuliert. Ich meine viel eher, dass eine saloppe Verwendung des Begriffs ein bestimmtes Verständnis von Ein- und Ausschluss nahelegt. Der Begriff Inklusion hat uns unbestritten einen Fortschritt in unserem Denken über Ausschluss und Eingliederung gebracht. Es geht nicht mehr darum, die eine homogen konstruierte Gruppe, nämlich die der behinderten Menschen, in eine andere homogen konstruierte Gruppe, nämlich die der nicht behinderten Menschen, zu integrieren. Wenn wir von Inklusion sprechen, gehen wir von einer grundsätzlichen Verschiedenheit und Heterogenität der Individuen aus. Es soll ein Raum geöffnet werden, in dem die Individuen in ihrer Verschiedenheit zusammenkommen können, in welchem sie zusammen lernen und etwas Gemeinsames schaffen können. Dennoch meine ich, dass eine zumindest unbedachte Verwendungsweise des

1 Bei diesem Beitrag handelt es sich um eine von Heike Henning bearbeitete und transkribierte Version des Eröffnungsvortrags von Michael Turinsky. Daher wurde auf die sonst übliche Zitation verzichtet. 
Begriffs Inklusion die Gefahr birgt, die Vorstellung nahezulegen, dass der Ausschluss bestimmter Menschengruppen quasi ein zufälliger wäre oder etwas, das darauf zurückzuführen wäre, dass die Menschen einfach so dumm oder moralisch verwerflich wären, dass sie andere nicht in etwas einbeziehen. Man kann nun hingegen zeigen, dass jedes Feld, jede Institution durch bestimmte Ausschlüsse konstituiert ist - strukturell, wie wir sagen würden. Ich gebe Ihnen ein Beispiel: Es ist die Rede davon, dass so wenige behinderte Menschen am Arbeitsmarkt teilhaben können. Jetzt könnte man auf die Idee kommen: Naja, ist halt leider so, das müssen wir nun ändern. Was aber dabei unterschlagen wird, ist natürlich die Tatsache, dass der Arbeitsmarkt durch seine Einbettung in kapitalistische Produktionsverhältnisse notwendigerweise Ausschlüsse produziert. Mit anderen Worten: Wirklicher Einschluss, wirkliche Inklusion kann nur durch eine radikale Transformation des ausgrenzenden Feldes oder der ausgrenzenden Institution geschehen.

Der dritte Punkt weshalb ich nicht mehr von Inklusion spreche, betrifft die darin implizierte Aufteilung der Wirkungsmacht: Die inklusive Geste, nämlich die des Hereinholens, ist immer eine, die von nicht behinderten Akteur ${ }^{*}$ inn)en ausgeht. Wir behinderte Menschen fungieren dabei weniger als aktiv Handelnde, sondern als Personen, die diese inklusive Geste wohlwollend und dankend entgegennehmen. Auch deshalb bin ich dazu übergegangen, nicht mehr so viel von Inklusion zu sprechen. Vielmehr bezeichne ich meine eigene künstlerische Praxis als Crip Choreography. Eine kleine ironische Wendung, die aber durchaus ernsthaft gemeint ist.

\section{Crip Choreography}

Crip ist ein Begriff, der vor einigen Jahren von Robert McRuer geprägt wurde. McRuer schrieb vor ein paar Jahren ein sehr interessantes $\mathrm{Buch}^{2}$, in dem er im Prinzip versucht, zwei verschiedene Diskursstränge miteinander zu verbinden: Nämlich zum einen die sogenannte Queer Theory und zum anderen die Disability Studies.

Queer war zunächst ein negativ konnotierter Begriff, mit dem schwule und lesbische Menschen bezeichnet wurden. In der Queer Theory wurde diese abschätzige Bezeichnung aufgegriffen und für eine kritische Auseinandersetzung mit dem, was in der Queer Theory als Heteronormativität und als Zweigeschlechtlichkeit bezeichnet wird, fruchtbar gemacht. Queer Theory versucht, die gesellschaftlichen Machtverhältnisse zu hinterfragen, die den Zwang

2 Bei dem hier erwähnten Buch handelt es sich um McRuer, R. (2006). Crip Theory: Cultural Signs of Queerness and Disability. New York: New York University Press. 
zur Heterosexualität und Zweigeschlechtlichkeit (re-)produzieren. McRuer hat analog hierzu den zunächst abschätzigen Begriff crip (engl. für Krüppel) aufgenommen, ihn positiv gewendet und für eine kritische Auseinandersetzung mit dem, was er als Compulsary Able-Bodiness bezeichnet - auf Deutsch könnten wir vielleicht sagen zwanghafte Funktionstüchtigkeit - verwendet.

Der Begriff Crip, wie ich ihn gebrauche, markiert eine spezifische Widerständigkeit gegen ebenjene zwanghafte Funktionstüchtigkeit des Körpers. Zwei Aspekte hierzu erscheinen mir zentral: Auf der einen Seite, wenn ich von Widerständigkeit gegen die Funktionstüchtigkeit spreche, versuche ich, Behinderung relational zu verstehen. Die Widerständigkeit des Körpers ist nur in Bezug darauf zu verstehen, wogegen sie widerständig ist. Und auf der anderen Seite möchte ich die Materialität der Widerständigkeit hervorheben. Es geht nicht um die Absicht, widerständig zu sein, sondern der Körper in seiner Materialität ist so.

Choreografie kann nun zunächst, etwa im Anschluss an die klassische Definition Doris Humphreys, als Kunst, Tänze zu machen, bezeichnet werden. Vor dem Hintergrund aktueller Debatten schlage ich indes eine andere Akzentuierung vor: Choreografie als ein Werkzeugkoffer, in dem lauter verschiedene Werkzeuge sind, mit denen man etwas ganz Bestimmtes machen kann. Man kann bspw. eine raumzeitliche Bewegungsorganisation analysieren und konstruieren. Hier liegt also der Fokus auf der Analyse und Konstruktion von (raumzeitlicher) Bewegungsorganisation.

Und jetzt führe ich diese beiden Aspekte, nämlich Crip als Widerständigkeit gegen zwanghafte Funktionstüchtigkeit und Choreografie als Praxis der Analyse und Konstruktion einer raumzeitlichen Bewegungsorganisation, zusammen. In dem, was ich als Crip Choreography bezeichne, geht es darum, diese Widerständigkeit des Körpers fruchtbar zu machen für eine Des- und Reorganisation des choreografischen Feldes. Es geht darum, körperliche Bewegung, aber eben auch ganz andere Dinge wie Sound, Licht, Kostüm neu zu verwenden, das heißt, deren Bewegung in einer Art und Weise zu organisieren, welche mit der Widerständigkeit des Körpers in Einklang steht. Es geht darum, die Materialität dieser Widerständigkeit des Körpers aufzugreifen und $\mathrm{zu}$ entfalten und in einer anderen Weise sicht- und lesbar werden zu lassen. Ich wende mich damit gegen eine Praxis, die Körper in eine vorgegebene, tradierte Bewegungsform presst, an die man sich anpassen soll. Ich entscheide mich bewusst dagegen, den Körpern eine überkommene Bewegungsorganisation aufzudrängen. Vielmehr gilt es, die eigene inhärente materielle Widerständigkeit der Körper zu entfalten. Eine Praxis, die ich als Crip Choreography bezeichne, besteht darin, solche tradierten Bewegungsorganisationen zu desorganisieren und durch neue Organisation des Bewegungsmaterials zu ersetzen. 
Jetzt kann man sich berechtigterweise fragen, ob es in der Choreografie nur um diese eigenartige Widerständigkeit des Körpers geht, um dieses komische formale Hin- und Herorganisieren? Keineswegs! Choreographische Praxis setzt natürlich immer auch so etwas wie Sinn (gleich in zweifacher Hinsicht) in Szene: Auf der einen Seite geht es immer um einen Sinn, der beispielsweise durch ein Thema, eine künstlerische Intention, Stimmungen, eine Gestimmtheit, ein Begehren oder mehrere Wünsche angezeigt ist. Daneben geht es aber immer auch um den Eigensinn des Körpers. Dieser widersetzt sich vielleicht mal dem anderen Sinn - oder steht zu ihm in Resonanz. Für mich gilt es, in der choreographischen Praxis ein produktives Spannungsverhältnis zu entfalten. Nämlich zwischen dem, was ich mit einem Thema intendiere, und dem Eigensinn, den der Körper mitbringt. Manchmal ist das Verhältnis zwischen den beiden Sinnkomponenten ein Resonanz-, manchmal ein Differenzverhältnis. Es geht jedoch immer darum, diese beiden Sinnmomente miteinander ins Spiel zu bringen.

Neben Eigensinn des Körpers und thematisch intendiertem Sinn möchte ich das Thema der Organisation nochmals aufgreifen.

\section{Organisation des Materials in der Zeit}

Bekanntlich stellt sich das Formproblem in den zeitbasierten Künsten vor allem als ein Problem der Organisation des Materials in der Zeit. Dieses stellt sich natürlich besonders dort, wo sich die Widerständigkeit des Körpers in einem spezifischen Verhältnis zur Zeit zeigt. Eine Reihe von Autorinnen und Autoren haben den Begriff Crip Time geprägt. Manche Körper funktionieren langsamer. Gleichzeitig geht es nicht nur um Langsamkeit, sondern auch um so etwas wie eine vorrangige Tendenz zur Wiederholung gegenüber der Variation. Bestimmte Körper sind eher zur Wiederholung geneigt als zur virtuosen Variation. Denken Sie einmal über den Ausdruck intakte Körper nach. In diesem Begriff steckt das Wort Takt. Und manche Körper widersetzen sich eben genau diesem Intaktsein.

Mich hat in der choreografischen Arbeit immer besonders interessiert, wie ich vor dem Hintergrund dieser Überlegungen mit der Forderung nach Komplexität in der Form und der Organisation des Materials umgehe. Dazu habe ich verschiedene Strategien entwickelt: Eine Strategie könnte ich etwa als crossmediale Variation bezeichnen. In meinem ersten Solo heteronomous male ${ }^{3}$ habe ich mich mit dem Thema Männlichkeit beschäftigt. Ich habe das Prin-

3 Dieses Stück kann unter https://vimeo.com/97516408 angeschaut werden [28.06.2020]. 
zip des Umkippens verwendet. Dieses formale Prinzip eines Wechsels von der Vertikalität in die Horizontalität habe ich durch verschiedene Medien durchdekliniert. Das Ganze beginnt damit, dass mich jemand in meinem Rollstuhl nach hinten umkippt. Dann liege ich umgekippt im Rollstuhl und schäle mich da langsam heraus. Später kehrt dieses Prinzip der Horizontalität und Vertikalität wieder zurück, indem ich mit Wackelpuppen spiele, die, wenn man sie anstößt, eine Zeit lang herumwackeln und dann wieder in der Vertikalen zum Stehen kommen. Dann spreche ich auch über diese Vertikalität und ihre phallischen Implikationen. So dekliniere ich eben dieses Prinzip durch verschiedene Medien hindurch.

Eine andere Strategie, die ich erwähnen möchte und die etwa für meine Arbeit Reverberations ${ }^{4}$ wichtig war, ist das, was ich Textur nenne. Ich gebrauche diesen Begriff als einen Aspekt von Form. Es gibt sozusagen eine Makroebene, das sind die groben kompositorischen Strukturen. Daneben gibt es aber auch eine Mikroebene, die Textur. In Bezug auf die Organisation des Materials in der Zeit geht es mir um alle jene kleinen Verschiebungen in einem Körper, zwischen den Körpern und dem Sound. Durch diese minimalen Verschiebungen, durch dieses nicht vollständige Synchronisiert-Sein ergibt sich eine Qualität, die ich als Textur bezeichne. Der Körper, von dem ich dabei ausgehe, ist, wie man sagen könnte, vor allem ein synkopischer Körper, ein OffBeat-Körper. Die Konsequenzen, die man daraus ziehen kann, sind komplex. Ich meine: Wir brauchen die Lust am Beat nicht zu negieren. Vielmehr geht es darum, das spannungs- und lustvolle Spiel zwischen Beat und Off-Beat beherzt anzugehen!

4 Diese Produktion kann unter https://vimeo.com/255534200 angesehen werden [28.06.2020]. 



\section{Zur gesellschaftlichen Inklusion kulturell-künstlerischer Repräsentationen von Behinderung}

\section{Einleitung}

In diesem Beitrag wird aus sozial- und erziehungswissenschaftlicher Perspektive nach den kulturell-künstlerischen Repräsentationsformen von Behinderung gefragt. Es wird zunächst ein Einblick in Erkenntnisse der Disability Studies zur Darstellung von Menschen mit Behinderungen in Kunst und Kultur gegeben, nachfolgend menschenrechtspolitische und pädagogische Entwicklungen hin zu inklusiver Bildung aufgezeigt woraufhin zum Schluss kritische Gedanken zur Ästhetik sowie der Gestaltung von Musik- und Tanzpädagogik formuliert werden.

Der Beitrag zielt darauf ab, den interdisziplinären Austausch zwischen den Disability Studies und der kulturellen Bildung zu vertiefen und bei der Beschäftigung mit Fragen nach gesellschaftlicher Inklusion künstlerische Ausdrucksformen einzuschließen.

\section{Ausgangsbeobachtungen und kurze Geschichte des ,Blicks auf Behinderung}

Ich beginne mit der Ausgangsbeobachtung und -these, dass Menschen mit Behinderung in Medien, Kultur und Kunst nicht ausreichend repräsentiert sind. Sie treten als Adressat( ${ }^{*}$ inn)en und Produzent $\left({ }^{*}\right.$ inn)en nicht gleichberechtigt auf und werden medial oft als Randerscheinung und künstlerisch, wenn überhaupt, dann nach wie vor meist als Ausnahmeerscheinungen behandelt.

Im interdisziplinären Forschungsfeld der Disability Studies wird ergründet, wie es zu einem solchen Blick auf Behinderung kommt. Zu nennen sind hier insbesondere die grundlegenden Untersuchungen von Tobin Siebers (2009), der die These entwickelte, dass Kunst die Empfindungen von Körpern in Anwesenheit anderer Körper zentriert. Das heißt, künstlerische Darstellungen fordern uns zur Wahrnehmung der eigenen Körperlichkeit und der der anderen auf. Bei der Rezeption von Kunst greifen wir auf gewohnte, für uns typische Deutungsweisen und Wahrnehmungsmuster zurück, die dann durch die künstlerische Darstellung bestätigt oder irritiert werden können. Unser ästhetisches Empfinden spiegelt deshalb - so Siebers - unser Verständnis von der 
Welt und ist von alltäglichen „politischen Haltungen wie Akzeptanz oder Ablehnung kaum zu trennen“ (Siebers, 2009, S. 20).

Dass der Fokus in der Repräsentation von Behinderung in Medien und Kultur auf Extraordinaries, d. h. auf den Ausnahmeerscheinungen, liegt, ist also als ein soziales Phänomen und durch gesellschaftliche Marginalisierung und soziale Abwertung zu erklären (vgl. Garland-Thomson, 2004).

Mein Innsbrucker Kollege Volker Schönwiese hat anhand des im Schloss Ambras (Innsbruck) präsentierten Bildnis eines behinderten Mannes gezeigt, dass Darstellungen von körperlichen Beeinträchtigungen bereits im 16. Jahrhundert die Neugier des Publikums nach dem Fremden, dem Kuriosen stillen sollten (vgl. Schönwiese \& Mürner, 2005). ${ }^{1}$ Auch in den Freak Shows, die sich im 19. Jahrhundert großer Beliebtheit erfreuten, wurden Menschen mit Behinderungen immer wieder zum Objekt der Sensation - und versicherten zugleich allen anderen ihre Zugehörigkeit zu den Normalen und/oder (körperlich) Gesunden. Für das 20. Jahrhundert erarbeitete Rosemarie GarlandThomson im Zuge ihrer feministischen Überlegungen zu den Disability Studies eine Systematik der typischen (visuellen) Darstellung von behinderten Menschen in Medien und Kunst: Sie unterscheidet zwischen wundersamen, gefühlvollen, exotischen und realistischen Darstellungen. Diese führt sie auf die Betrachtungsweisen von Behinderung durch die Mehrheitsgesellschaft zurück, also darauf, was moderne Bürgerinnen in Behinderung sehen wollen. Den Diskurs dominieren der bemitleidende Blick, der bewundernde Blick und der (meist medizinisch interessierte) normalisierende Blick (vgl. GarlandThomson, 2004). Eine aktuelle Studie aus Österreich zur Repräsentation von Menschen mit Behinderungen in den Medien zeigt, dass diese Bilder bis heute in der Mehrzahl sind. Im Jahr 2015/2016 untersuchte die Autorin Maria Pernegger im Auftrag von Media Affairs (Wien) fast 600 Text- und Bildbeiträge über Menschen mit Behinderungen aus österreichischen Massenmedien. Dabei ist anzumerken, dass insgesamt wenig berichtet wird und die Beiträge zumeist im Kontext Charity oder Sport angesiedelt sind. Rund 20\% der Texte sind Held( ${ }^{*}$ inn)enerzählungen ebenso viele Opferdarstellungen. Nur ca. $60 \%$ der Beiträge zählt Pernegger zu den neutralen Darstellungen. Wenn behinderte Personen überhaupt zu Wort kommen, dann als Einzelpersonen. Kulturelle und politische Zusammenhänge oder Entwicklungen zum Thema werden kaum dargestellt (vgl. Pernegger, 2017).

1 Dieses Bild ist sehenswert, denn es trägt noch die Spuren eines ehemals angebrachten Vorhangs, der den Körper des Mannes verdeckte und von Betrachte$\mathrm{r}{ }^{\star}{ }^{*}$ inne) $n$ selbst angehoben werden musste. Zudem wurde das partizipatorisch angelegte Forschungsprojekt verfilmt (Verfügbar unter: https://www.youtube.com/ watch?v=A_bFO7fxIkU\&t=4s [02.05.2020]). 
Aus Sicht der Disability Studies ist an dieser Stelle zu betonen: Weder das in dieser Studie zitierte, bedürftige arme Hascherl noch der seine oder ihre Beeinträchtigung durch übermenschliche Kräfte überkommende Supercrip treffen den Punkt bei der Frage danach, wie Behinderung angemessen repräsentiert werden kann. Es geht vielmehr darum, vielfältige Körper, Ausdrucksweisen und Lebensrealitäten wahrzunehmen und zugänglich zu machen; also zu verstehen, was als Behinderung erfahren wird, um soziale Inklusion und kulturelle Partizipation zu ermöglichen.

\section{Zur menschenrechtlichen Perspektive auf Behinderung}

Wenn Kinder, Jugendliche und Erwachsene mit körperlichen und/oder geistigen Beeinträchtigungen Unterstützung benötigen, um ihren Alltag zu bewältigen oder wenn Unterstützung in der Pflege oder bei Entscheidungsfindungen benötigt wird, dann heißt das nicht, dass die betroffenen Personen nicht gleichermaßen das Recht auf Bildung, Arbeit, Familie, Kunst und Kultur besitzen. Aus menschenrechtlicher Perspektive ist Behinderung als Wechselwirkung zwischen einer Beeinträchtigung und Barrieren in der Umwelt zu verstehen (vgl. UN-Behindertenrechtskonvention ${ }^{2}$ Artikel 3): Soziale Ausgrenzung und fehlende angemessene Vorkehrungen für die Teilhabe aller bringen Behinderungen erst hervor. Dies gilt im Prinzip für alle Bevölkerungsgruppen, die aufgrund ihrer Gruppenzugehörigkeit mit Nachteilen oder Stereotypen zu kämpfen haben.

Dies möchte ich kurz an einem anderen, historischen Beispiel verdeutlichen. Noch vor rund 100 Jahren wurden Frauen und Mädchen von höherer Bildung ausgeschlossen, weil angenommen wurde, Frauen besäßen als Angehörige des schwachen Geschlechts nicht die (biologischen) Fähigkeiten, um bspw. zu studieren. Die damals häufig diagnostizierte Hysterie stellt aus heutiger Perspektive den Versuch dar, die soziale und rechtliche Abhängigkeit von Frauen aufrechtzuerhalten und gesellschaftlich zu legitimieren. Zu einer gleichberechtigten Repräsentation und körperlichen Selbstbestimmung gelangten Frauen erst im Zuge der Anerkennung umfassender Personen- und Freiheitsrechte. Menschen mit Behinderungen befinden sich in einer vergleichbaren Position.

Wie bedeutsam eine wirkliche Gleichstellung für die Lebenssituation behinderter Menschen und unser Selbstverständnis als demokratische Gesellschaft ist, haben die heftigen und kontroversen bildungspolitischen Debatten

2 Die UN-Behindertenrechtskonvention ist verfügbar unter https://www.behinderten rechtskonvention.info/uebereinkommen-ueber-die-rechte-von-menschen-mit-be hinderungen-3101/ [02.05.2020]. 
gezeigt, die mit der Ratifizierung der 2006 verabschiedeten UN-Konvention über die Rechte von Menschen mit Behinderungen ausgelöst wurden. In der UN-BRK wurde erstmals das Recht auf Inklusion definiert und ein diskriminierungsfreier Zugang zu Bildung festgelegt. Seitdem besteht eine breitere öffentliche Aufmerksamkeit gegenüber der benachteiligten Situation, in die Menschen gebracht werden, die in Heimen, Einrichtungen, Sonderschulen oder Werkstätten leben, lernen oder arbeiten. Einrichtungen, die aus erziehungswissenschaftlicher Perspektive die Möglichkeit von Bildungsprozessen, persönlicher Entwicklungen und Selbstbestimmung strukturell begrenzen oder nicht gewährleisten. Solange einem Teil der Kinder und Jugendlichen Teilhabe an Bildung und Kultur verwehrt wird, verwandeln sich ihre ungleichen sozialen Ausgangsbedingungen und körperlichen sowie geistigen Voraussetzungen in Benachteiligungen. Ich spreche hier explizit nicht nur von körperlichen Beeinträchtigungen, wie beispielsweise schwer hören zu können oder kognitiven Problemen, sondern schließe Beeinträchtigungen, die mit sozialen und ökonomischen Nachteilen einhergehen, mit ein.

In den Bildungswissenschaften belegen Untersuchungen, dass Separation und Segregation in Sonderschulen oder Spezialklassen das Phänomen Behinderung erst hervorbringen (vgl. Powell, 2016). Kinder und Jugendliche, die aufgrund ihrer Herkunft oder einer Beeinträchtigung nicht an allgemeinen Bildungsangeboten partizipieren, werden auf ein spezielles Merkmal festgelegt; sie werden pädagogisch dazu aufgefordert, sich zu schonen oder schützen zu lassen, sich in abhängige Subjektpositionen zu begeben und möglichst keine Erwartungen auf gesellschaftliche Partizipation oder körperliche Selbstbestimmung zu artikulieren (vgl. Pfahl 2010, 2011).

\section{Inklusive Bildung}

Ein zentrales Ziel und Prinzip der inklusiven Pädagogik ist deshalb der volle und gleichberechtigte Zugang zu hochwertiger Bildung - hier insbesondere zur Teilhabe an einem gemeinsamen Unterricht, der in schulischen sowie außerschulischen Bildungsangeboten zu fördern ist. Im gemeinsamen Unterricht haben alle Kinder und Jugendliche die Möglichkeit, sich einzubringen, Interessen $\mathrm{zu}$ entwickeln und ihnen nachzugehen. Sie lernen, ihre (körperlichen) Gemeinsamkeiten und Unterschiede wahrzunehmen und zu thematisieren und werden durch pädagogisches Fachpersonal darin unterstützt, mit geeigneten Lernmaterialien ihre Fähigkeiten zu erweitern. In der inklusiven Bildung stellen dabei sowohl die Fragen nach der Teilhabe (participation) als auch nach dem Lernerfolg (achievement) wichtige Leitprinzipien dar, die bereits 1994 durch die UNESCO formuliert wurden (vgl. UNESCO, 1994). Ge- 
meinsamer Unterricht bedeutet also nicht den Verzicht auf Lernerfolg oder Leistung. Vielmehr werden gemeinsam mit dem Kind und seinen Erziehungsberechtigten die Interessen erkundet und Lernziele festgelegt. Dass Kinder unterschiedliche Lernziele verfolgen, bereichert die Gruppe ebenso wie die Diversität ihrer Herkunft und Fähigkeiten. Die Teilhabe, d.h. das Interesse und die Möglichkeit sich einzubringen, wird dabei berücksichtigt.

Ohne spezifische musik- oder tanzdidaktische Fragen zu vertiefen, möchte ich an dieser Stelle einen häufig geäußerten Einwand vorwegnehmen nämlich, dass sich Pädagog( ${ }^{*}$ inn)en oft nicht zuständig oder kompetent fühlen, inklusiven Unterricht durchzuführen, weil sie keine sonderpädagogische Ausbildung besitzen und zudem annehmen, Bildungsangebote für behinderte Menschen müssten einen therapeutischen Mehrwert besitzen. Dies verwundert mich immer wieder aufs Neue. Jede Pädagogin und jeder Pädagoge ist qua Ausbildung befähigt, Kinder, Jugendliche oder Erwachsene zu unterrichten. Meist sind Ausbildungsgänge auf Fächer (oder spezifischer: auf musikoder tanzpädagogische Angebote wie ein bestimmtes Instrument, einen Tanzstil etc.) und das Alter der Edukant( ${ }^{*}$ inn)en spezifiziert. Diese Befähigung wird dann im Unterricht immer wieder irritiert oder verwandelt und muss erneuert werden. So auch in der Musik- und Tanzpädagogik. Kinder und Jugendliche mit Behinderungen sind in erster Linie Kinder und Jugendliche, sie sind Schüler*innen, und fallen damit in das Aufgabengebiet von (Musik-)Pädagog $\left({ }^{*}\right.$ inn)en. Meist spielen Fragen der Motivation, des Interesses oder nach den Lebensumständen eine weitaus wichtigere Rolle bezüglich der Überlegung, wie Unterricht zu gestalten ist, als die Frage danach, ob jemand eine Beeinträchtigung hat. In den meisten Ländern existieren zudem gar keine sonderpädagogischen Einrichtungen - Pädagog $\left({ }^{*}\right.$ inn)en entwickeln dort gemeinsame Lernangebote an allgemeinbildenden Schulen (vgl. Powell, 2016). Auch sind zuständige öffentlichen Institutionen der Kunst- und Kulturvermittlung aufgefordert, sich zu öffnen, den Zugang zu Angeboten möglichst barrierefrei $\mathrm{zu}$ gestalten und durch ein annehmbares, vielfältiges und attraktives Angebot Personen mit unterschiedlichsten Bedürfnissen zu erreichen, um gesellschaftliche Inklusion voranzutreiben. Dabei sind Menschen mit Behinderungen nicht nur als Adressat( ${ }^{*}$ inn)en von Bildungsangeboten mitzubedenken, sondern als Künstler*innen und Dozent( ${ }^{*}$ inn)en in die Gestaltung der Angebotsstrukturen und die Kulturvermittlung einzubeziehen. Ein solches Vorgehen entspricht der, mit dem menschenrechtlichen Anspruch auf Partizipation entwickelten 4-A-Scheme für (öffentliche) Institutionen. Demzufolge sollen Bildungsangebote available (verfügbar), accessible (zugänglich), acceptable (annehmbar) und adaptable (adaptierbar, flexibel) sein (vgl. Motakef, 2006, S. 27). 


\section{Fragen an die Musik- und Kunstpädagogik}

Anregungen für ein Mehr an Teilhabe in der Musik- und Tanzpädagogik möchte ich in Anlehnung an meinen Kollegen Alan Courtis, Musikpädagoge aus Buenos Aires, geben. Er hat im Januar 2016 in Innsbruck einen Vortrag gehalten und die Wichtigkeit dieses Themas verdeutlicht. Courtis stellte damals einige Fragen, die ich hier aufgreifen möchte: Wie beeinflusst der ästhetische und technische Wandel in Musik und Kunst, der sich im 20. Jahrhundert vollzogen hat, die Musik- und Kunstpädagogik? Inwiefern tragen neue Konzepte zur Überwindung falscher Mythen über Behinderung bei? Sind künstlerische Leistungen von behinderten Künstler(*inne)n zu erwarten oder handelt es sich bei Kunst/Musik und Inklusion nur um Therapieformen? Gibt es nur einen korrekten Weg, ein Instrument zu spielen? Was bedeutet es, out of tune zu sein? Geht es nur um Spieltechnikvermittlung? Können wir etwas lernen von behinderten Künstler( ${ }^{*}$ inne)n? Welche pädagogischen Paradigmen haben sich in der Musik entwickelt? Welche Rolle nimmt das Publikum ein? Welche Verantwortung hat die Gesellschaft? Wie kann Inklusion in Musik und Kunst gelingen?

Ich denke, wir sollten uns nicht die Chance nehmen lassen, Fragen an die Ästhetik, an die Kunst und an die Organisation von Kunst zu stellen - und zwar aus inklusiver Perspektive. Denn gerade Musik und Tanz sind Bereiche, die klassisch praktiziert, extrem hierarchisch organisiert sind. Man darf die Frage stellen, warum das so ist und welche Folgen das für die Vorstellung des künstlerisch Wertvollen und des Genusses von Kunst hat.

Eine Konsequenz des Hochleistungsparadigmas, welches nur bestimmte richtige Bewegungen oder richtige Töne kennt, ist sicherlich, dass eine große Distanz zwischen Kunst und Publikum geschaffen wird. Die Sphäre des Künstlerischen bleibt so einigen wenigen vorenthalten und es wird suggeriert, nur sie wüssten um die richtige Ausübung von Musik und Tanz. Bei der Beschäftigung mit Inklusion und Behinderung geht es also nicht nur um die Erweiterung des Kreises der Adressat(*inn)en von Musik und Kunst, sondern um eine Erweiterung des künstlerisch Relevanten, d.h. um eine Erneuerung unserer Ästhetik. In der Popularmusik gibt es solche Entwicklungen bereits (vgl. Becker, Knöppchen \& Pfahl, 2016). In der Klassik und der traditionellen Musik ist das meines Erachtens (noch) nicht öffentlich wahrnehmbar. Inklusion für einen Wandel in der Ästhetik zu nutzen und Kunst als Inklusion zu praktizieren, das ist ein äußerst spannendes Vorhaben, für das Beispiele von Performance-Künstler( ${ }^{*}$ inne $) n$ und Aktivist $\left({ }^{*}\right.$ inn)en vorliegen. Dazu sollten wir „[e]in auf Harmonie, körperliche Ganzheit und nicht zuletzt Gesundheit gegründetes Kunstverständnis“ verwerfen und den „ästhetischen Wert, der Be- 
hinderung [...] zugewiesen wird, positivieren“ (Siebers, 2009, S. 15, Hervorh. L.P.).

\section{Literatur}

Becker, C., Knöppchen, F. \& Pfahl, L. (2016). The world's first amputee pop artist. Darstellung von Behinderung in Viktoria Modestas ,Prototype. PopScriptum [Sound, Sex und Sexismus], 12. Verfügbar unter: https://edoc.hu-berlin.de/ bitstream/handle/18452/21075/pst12_becker_knoeppchen_pfahl.pdf?sequen ce $=1$ \&isAllowed $=y[02.05 .2020]$.

Garland-Thomson, R. (2004). Integrating Disability - Transforming Feminist Theory. In B. G. Smiths \& B. Hutchison (Hrsg.), Gendering Disability (S. 73-103). New Brunswick, NJ: Rutgers University Press.

Motakef, M. (2006). Das Menschenrecht auf Bildung und der Schutz vor Diskriminierung. Exklusionsrisiken und Inklusionschancen. Berlin: Deutsches Institut für Menschenrechte.

Pernegger, M. (2017). Menschen mit Behinderungen in österreichischen Massenmedien. Jahresstudie 2015/16. Wien: Media Affairs.

Pfahl, L. (2010). Organisierte Armut. Soziale Ausgrenzung im gegliederten Schulsystem. WZB-Mitteilungen, 128, 11-14.

Pfahl, L. (2011). Techniken der Behinderung. Der deutsche Lernbehinderungsdiskurs, die Sonderschule und ihre Auswirkungen auf Bildungsbiografien. Bielefeld: transcript Verlag. https://doi.org/10.14361/transcript.9783839415320

Powell, J. J. W. (2016). Barriers to Inclusion. London: Paradigm Publisher. https:// doi.org/10.4324/9781315635880

Schönwiese, V. \& Mürner, C. (2005). Das Bildnis eines behinderten Mannes. Kulturgeschichtliche Studie zu Behinderung und ihre Aktualität. Psychologie und Gesellschaftskritik, 113 (1), 95-125. Verfügbar unter: http://bidok.uibk.ac.at/li brary/schoenwiese-bildnis.html [02.05.2020]. Verfilmung des Forschungsprojektes abrufbar unter: https://www.youtube.com/watch?v=A_bFO7fxIkU\&t=4s [02.05.2020].

Siebers, T. (2009). Zerbrochene Schönheit. Essays über Kunst, Ästhetik und Behinderung. Bielefeld: transcript Verlag. https://doi.org/10.14361/9783839411322

UNESCO (1994). Salamanca-Erklärung und der Aktionsrahmen zur Pädagogik für besondere Bedürfnisse. Verfügbar unter: https://www.unesco.de/sites/default/ files/2018-03/1994_salamanca-erklaerung.pdf [02.05.2020]. 



\section{Frictions and Fractions?!}

\section{Kritische Perspektiven auf Kulturarbeit, Kulturvermittlung und Diversity ${ }^{1}$}

Kulturelle Teilhabe ist in der Allgemeinen Erklärung der Menschenrechte (Artikel 27) ${ }^{2}$ als Menschenrecht verankert. Eine Vielzahl von Menschen wird jedoch nicht von öffentlich geförderten kulturellen Angeboten erreicht und hat auch nicht die Möglichkeit, selbst künstlerisch oder kulturell produzierend tätig zu sein bzw. wahrgenommen zu werden. Ausschlüsse oder Barrieren in sowie durch Kunst und Kultur sind weit verbreitet und wirkmächtig. Doch welche Strukturen oder Mechanismen fördern Ausschlüsse? Wie können sie systematisch eruiert, benannt und analysiert werden? Wie lassen sich Forderungen nach Teilhabe, nicht diskriminierenden Sprachpolitiken und Öffnung von Institutionen tatsächlich einlösen? Und wodurch können Veränderungen in Richtung einer transformativen, emanzipatorischen und solidarischen Arbeit in Kunst und Kultur erzielt werden? Ausgehend von Kritischer Kulturvermittlung ${ }^{3}$ und Kritischem Diversity ${ }^{4}$ überlegen wir in diesem Gespräch, wie Kulturarbeit als kritische Praxis konzeptualisiert und umgesetzt werden kann.

1 Dieser Beitrag ist der überarbeitete Vortrag bei der Tagung All inclusive?!, der auf unserem gleichnamigen Artikel im eJournal p/art/icipate \#9 Open Up! Ein- und Ausschlüsse in Kunst und Kultur (verfügbar unter: www.p-art-icipate.net/ejournalhome [17.07.2020]) aufbaute. Beim Vortrag ließen wir im Hintergrund eine PowerPoint-Schleife mit aktivistischen Statements zum Thema laufen, die leider aus editorischen Gründen keinen Eingang in diesen Text finden konnten.

2 Vgl. dazu https://www.menschenrechtserklaerung.de/kultur-3689/ [17.07.2020].

3 Kritische Kulturvermittlung, wie sie u. a. von Carmen Mörsch (vgl. Mörsch, Schade \& Vögele, 2018; Mörsch \& Forschungsteam, 2009) theoretisiert wurde, baut auf einem emanzipatorischen Verständnis von Pädagogik auf und ist als Vermittlungspraxis zu verstehen, die affirmative Praktiken der Kunst- und Kulturvermittlung sowie Normen und hegemoniale Ordnungen im Kunst- und Kulturfeld hinterfragt und intervenierend in Kunst- und Kultureinrichtungen eingreift. Ziel ist es, (selbst-)kritische Reflexionsräume zu schaffen und letztlich eine Veränderung der Institutionen zu erwirken.

4 Der Begriff Kritisches Diversity bezieht sich auf einen spezifischen Diversity-Ansatz des Wiener Vereins ][diskursiv. Er basiert auf Erfahrungswissen sowie aktivistischen Wissensständen und Praktiken. Im Mittelpunkt des Ansatzes stehen machtund diskriminierungskritische sowie aktivistische Zugänge (vgl. Frketić, 2014; Baumgartinger \& Frketić, 2019). 
Dieser Auseinandersetzung ist eine gemeinsame Veranstaltungsreihe $\mathrm{zu}$ Kulturarbeit und Diversity ${ }^{5}$ am Programmbereich Zeitgenössische Kunst und Kulturproduktion des Kooperationsschwerpunktes Wissenschaft und Kunst (Paris-Lodron-Universität und Universität Mozarteum Salzburg) vorausgegangen. Dabei haben wir festgestellt, dass eine konkrete Verbindung von Kritischer Kulturvermittlung - als zentralem Teil von Kulturarbeit - und Kritischem Diversity wichtig ist, um eine allgemeinere Perspektive für eine Kulturarbeit als kritische Praxis zu entwickeln.

\section{Diversity und Kulturvermittlung}

Anita Moser (AM): Kulturarbeit lässt sich als ein Feld von Tätigkeiten bezeichnen, die im Zusammenhang mit der Programmgestaltung, Organisation und Kommunikation von Kunst und Kultur anfallen. Sie umfasst also theoretischinhaltliche Arbeiten gleichermaßen wie Umsetzungsprozesse in Institutionen, Profit- und Non-Profit-Einrichtungen und findet in großen Häusern ebenso wie bei der Umsetzung von kleineren Projekten statt. Kritische Kulturarbeit vollzieht sich vielfach im Bereich der sogenannten freien Kulturarbeit, welche im Kontext gesellschaftlicher und politischer Umbrüche der 1970er Jahre entstand und im Vergleich zu etablierter Kunst und Kultur weitgehend unterfinanziert ist. Aus Perspektive der Kulturbetriebslehre wird sie auch unter dem Begriff des Non-Profit-Sektors subsumiert. Beweggründe für die Gründung freier Initiativen waren insbesondere das Bedürfnis nach künstlerisch-kultureller Selbstorganisation, kreativem Selbstausdruck im Sinne einer Soziokultur, inhaltlicher Unabhängigkeit sowie nach gesellschaftlichem und politischem Engagement (vgl. Moser, 2015).

Ein wesentlicher Teil von Kulturarbeit ist die Kulturvermittlung, bei der es auf einer sehr allgemeinen Ebene darum geht, zwischen Kunst/Kultur und unterschiedlichen Publika zu vermitteln. Kulturvermittlung wird als Verständnishilfe zwischen Kunst und Publikum bezeichnet, wobei es laut Birgit Mandel auch darum geht, die besonderen Stärken der Künste für das Zusammenleben im Alltag zu nutzen. Diese liegen ihr zufolge darin, dass Künste kommunikative Prozesse in Gang setzen, die Wahrnehmung auf das Gewohnte verrücken und zeigen, dass alles ganz anders sein könnte (vgl. Mandel, 2005, S. 16). Kulturvermittlung fokussiert also auch auf die Veränderung von Sichtweisen und Einstellungen.

5 Diese fand im Zuge der Lehrveranstaltungen $P R$ in Kunst und Kultur von Anita Moser und Diversity in Kunst und Kultur von Persson Perry Baumgartinger im Wintersemester 2018/19 statt. 
Besonders in der Kritischen Kulturvermittlung (vgl. Mörsch, Schade \& Vögele, 2018; Mörsch \& Forschungsteam, 2009) werden Transformationspotenziale thematisiert, wobei diese letztlich auf die Veränderung von Strukturen und Institutionen abzielen Für Carmen Mörsch ist Kunstvermittlung eine Praxis, bei der Dritte eingeladen werden, Kunst und ihre Institutionen für Bildungsprozesse zu nutzen. Konkret heißt das auch, die Institutionen zu befragen, analysieren und dekonstruieren und, falls möglich, zu verändern, um sie dadurch „auf die eine oder andere Art fortzusetzen“ (Mörsch, 2009, S. 9). Hierbei ist der Aspekt von Transformation wichtig. Was verstehst du unter Diversity?

Persson Perry Baumgartinger (PPB): Diversity bzw. Diversität sind Begriffe, die einerseits häufig verwendet werden, andererseits als Konzepte meist inhaltsleer sind, also reine Papierarbeit bedeuten (vgl. Ahmed, 2011, 2012) und/oder zu einer Imagesache geworden sind (vgl. Schönefeld, 2017). Vlatka Frketić entwickelt bereits seit mehr als 15 Jahren eine Form des Kritischen Diversity, das im Rahmen des Vereins ][diskursiv ${ }^{6}$ und darüber hinaus ständig weiterentwickelt wird (vgl. Frketić, 2014; Baumgartinger \& Frketić, 2019). Diese Form des Kritischen Diversity bedeutet eine differenzierte und intersektionale Herangehensweise.

Der Ankerpunkt unseres Zugangs ist eine prinzipielle kritische Haltung im Sinne einer skeptischen Neugierde ${ }^{7}$, wobei Sprache und sprachliches Handeln sowie Diskriminierung ${ }^{8}$ und der Fokus auf Strukturen sehr wichtig sind, nicht nur die individuellen Handlungen. Diversity ist in diesem Verständnis ein Prozess, eine ständige Weiterentwicklung und Veränderung hin zu sozialer Gerechtigkeit, kein statisches Produkt, keine oberflächliche Imagepflege, sondern eine intensive Auseinandersetzung, die den jeweils Privilegierten auch mal wehtun kann. Es bedeutet eine parteiische Haltung, die Minderheiten und ihre Forderungen in den Mittelpunkt rückt und die (aktivistisches) Erfahrungswissen ernst nimmt. Ziel ist eine sozial gerechte(re) Gesellschaft mit Teilnahmeund Teilhabemöglichkeiten für all diejenigen, die im jeweiligen Moment Teil der Gesellschaft sind. Um dieses Ziel zu erreichen, braucht es einen Wechsel

6 Verein ][diskursiv - Verein zur Verqueerung gesellschaftlicher Zusammenhänge (Verfügbar unter: www.diskursiv.diebin.at [17.07.2020]).

7 Kritik bzw. kritische Haltung wird mit Ruth Wodak (in Kendall, 2007, Abs. 17) verstanden als: „[...] not taking things for granted, opening up complexity, challenging reductionism, dogmatism and dichotomies, being self-reflective in my research, and through these processes, making opaque structures of power relations and ideologies manifest. 'Critical', thus, does not imply the common sense meaning of 'being negative' - rather 'skeptical'. Proposing alternatives is also part of being 'critical.'.

8 Diskriminierung wird „als systematische Unterdrückung und Ausdruck einer gesellschaftlichen Ungleichheitsstruktur verstanden“" (Baumgartinger \& Frketić, 2019, S. 199). 
bezüglich der Diversity- und oft auch Antidiskriminierungsansätze - weg von Identitäten hin zu den dahinterliegenden Werten, Normen und Mächten. Sehr wichtig sind in diesem Konzept die vier Prinzipien Langsamkeit, Kritik, Respekt und Verantwortung, um kritische Denk- und Handlungsräume so zugänglich wie möglich zu machen. Ich denke, dass dieser Ansatz sehr fruchtbar sein kann für eine kritische Kulturarbeit (vgl. Baumgartinger \& Frketić, 2019).

Etwa das Modell der verschiedenen Ebenen als Analyse-, Reflexions- und Interventionstool, das hilft, Stereotypen, Diskriminierungen und unsichtbare (Gesellschafts-)Normen aufzudecken und an konkreten Situationen abzuarbeiten. Dabei wird meist von folgenden drei bis fünf Ebenen ausgegangen: der individuellen, sozialen und institutionellen Ebene (vgl. Frketić, 2014, S. 3233) bzw. der individuellen, interaktionalen, organisationalen, gesellschaftlichen und globalen Ebene (vgl. Bargehr \& Johnston-Arthur, 2018).

AM: Bei dem Modell der verschiedenen Ebenen geht es also darum zu analysieren, wie Diskriminierung auf welcher Ebene funktioniert?

PPB: Genauer gesagt, wie auf diesen Ebenen Diskriminierung hergestellt wird. Es soll also herausgearbeitet werden, welche Bedingungen etwa eine konkrete rassistische Situation in der Kulturvermittlung ermöglicht haben: Was war zwischen den einzelnen Personen, wer hat was gesagt oder getan? Wer war von Diskriminierung betroffen und wer hat davon profitiert? Wer hat geschwiegen und weggeschaut? Auf der institutionellen Ebene kann untersucht werden, inwiefern die Organisationsstruktur eines Museums z. B. dazu beigetragen hat, dass Diskriminierung möglich war und welche ungleichen Machtverhältnisse durch die Ausstellungsstücke und deren Vermittlung entstehen, aber auch, welche Gesetze im Hintergrund wirksam sind und Ähnliches. Auf der globalen Ebene kann herausgearbeitet werden, wie etwa kolonial gewachsene Strukturen und globale Ungleichheiten den Rahmen für diese rassistische Situation geboten haben.

AM: Wenn Diversity auf Kultureinrichtungen bezogen wird, geht es darum, das Konzept auf mindestens drei Ebenen zu verfolgen: Personal, Programm und Publikum (vgl. Aikins \& Gyamerah, 2016; Moser, 2019). Kritische Kulturvermittlung kann ein Ansatz sein, um beispielsweise die Programmebene von Kulturinstitutionen diskriminierungssensibel zu untersuchen und damit zusammenhängende Machtfragen zu diskutieren. Also etwa die Tatsache, dass Einrichtungen primär ausgehend von einem weißen Kulturbegriff und westlichen Ästhetiken programmieren, was wiederum die Ausblendung oder Exotisierung des Anderen nach sich zieht und eng mit der Frage zusammenhängt, wer in einer Institution Definitions- und Entscheidungsmacht hat.

PPB: Beim Kritischen Diversity würde der Verein ][diskursiv in deinem Beispiel über die Programmebene die Struktur in den Blick nehmen. Denn - mit Rückbezug auf Erkenntnisse der Trans und Queer Studies - kann der 
Blick verändert werden: Anstatt sich fixen Identitäten und (Fremd-)Zuschreibungen zuzuwenden, um sie im gleichen Zuge zu reproduzieren, gilt es, die dahinterliegenden Werte, Normen und Mächte in den Blick zu nehmen. Diese formen unsere Realität, eine Gesellschaft und ihre Institutionen unaufhörlich (neu) und setzen damit den Rahmen für strukturelle Ungleichheiten und Diskriminierung, einer weiteren wichtigen Kategorie des Kritischen Diversity. Also: Wie kann es möglich sein, dass bestimmte Menschen sichtbare Tätigkeiten haben und andere unsichtbare? Dass etwa weiße cis-Personen ${ }^{9}$ aus akademischen Familien in Organisationen sichtbarer und weiter oben in der Hierarchie sind und Schwarze, People of Color, trans Personen oder Menschen, die als behindert bezeichnet werden, weiter unten in der Hierarchie arbeiten und meistens nicht mit direktem Kontakt nach außen? Damit sind wir aber auch bei einem Dilemma, mit dem Diversity und Kulturarbeit zu tun haben: Wie kann mit dem Dilemma zwischen Selbstbestimmung und Fremdzuschreibungen umgegangen werden?

AM: Da es ohne Identitäten auch wieder nicht geht ...

PPB: Ja, besonders, weil wir ja meistens fremdbestimmen, also andere Leute in Kategorien einordnen. Dieses Einordnen hat Konsequenzen, weil wir in einem historisch gewachsenen, hierarchischen Gesellschaftssystem leben. Ich denke, dass sich dieses Dilemma nicht lösen lässt. Sogenannte Merkmale wie Hautfarbe, Kleidungsstil, Sprachverwendung etc. sind ja nicht per se gut oder schlecht, aber sie führen zu Diskriminierungen oder Privilegierungen, weil der gesellschaftliche Rahmen das möglich macht. Und das wäre dann quasi der Blick des Kritischen Diversity. Wenn ich eine Tour durch ein Museum gebe, die klassische Form der Kulturvermittlung, dann schätze ich die Leute vom ersten Moment an ein - das verändert mein ganzes Verhalten. Im Grunde könnte man da das Modell der verschiedenen Ebenen als Reflexionstool verwenden und eine Tour oder Performance reflektieren. Also untersuchen, wie und womit ich Stereotypen auflade und wie diese wiederum mein Verhalten verändern: Erwähne ich die Person oder nicht, lasse ich das Bild aus und gehe zu einem anderen oder nicht und welche Ausstellungsstücke muss ich in jeder Tour ansprechen, weil die Institution sie als Kanon definiert etc.? Gleiches gilt für die Musikpädagogik: Welches Stück nehme ich in mein Programm auf? Welches Lied spiele ich mit den Schüler $\left({ }^{*}\right.$ inne)n?

9 Cis bezeichnet eine Person, die sich mit dem bei der Geburt zugeschriebenen Geschlecht Mann oder Frau identifiziert. Cis ist - wie trans oder inter, aber auch nichtbinär - eine Form der Geschlechtlichkeit. Der Begriff wird auf Volkmar Sigusch' Neologismus Zissexualität zurückgeführt, den er 1991 als Gegenbegriff zu Transsexualität eingeführt hat, um zusätzlich zum pathologisierenden Begriff Transsexualität die als gesund geltende Norm Mann bzw. Frau sichtbar zu machen (vgl. Sigusch, 2013; vgl. Baumgartinger 2017). 
AM: In der Kritischen Kulturvermittlung geht es darum, solche Mechanismen sichtbar zu machen und anzusprechen, wie zum Beispiel ein Vermittlungsprojekt von Hansel Sato auf der documenta $12^{10}$ (vgl. Sato, 2009) zeigt: Er machte Führungen, wobei er sich mit jeweils unterschiedlichen Identitäten vorstellte, die Exponate aber immer mit gleichbleibenden Informationen erklärte. Anhand der Reaktionen und Fragen des Publikums wurde sichtbar, welche Erwartungshaltungen an ihn, an die Kunst und an die Kunstler*innen durch seine jeweilige (vorgegebene) Identität generiert wurden und welche Vorannahmen und Stereotypen dabei wirksam wurden. Im Anschluss an die Führungen diskutierte Hansel Sato sein Vorgehen und seine Beobachtungen mit dem teilnehmenden Publikum. Mit diesem Projekt dekonstruierte er auch das Vermittlungsformat Publikumsführung, da er nicht Expert(*inn)enwissen über die Werke vermittelte, sondern die gemeinsame Reflexion der Vermittlungssituation darüber ins Zentrum rückte.

Vermittlungsformate wie die traditionelle Publikumsführung, bei der Expertinnen und Experten von oben herab Wissen weitergeben, rechnet Carmen Mörsch (2009) in ihrer Untersuchung über Kunstvermittlung in Institutionen dem affirmativen Diskurs zu. Als einen weiteren nennt sie den reproduktiven Diskurs, bei dem u. a. in speziellen Workshops bestimmte Zielgruppen (Schulklassen, Lehrpersonen, Familien etc.) als Publikum von morgen herangebildet werden. Beide Diskurse kritisieren das System nicht, sondern wiederholen und stabilisieren es. Darüber hinaus führt Mörsch den dekonstruktiven und den transformativen Diskurs an, die beide institutionskritisch und im Kontext einer Kritischen Kulturvermittlung zentral sind. Im dekonstruktiven Diskurs werden gemeinsam mit dem Publikum Kanonisierungsprozesse sowie Einund Ausschlüsse hinterfragt, die in und durch Institutionen wirksam sind. Beim transformativen Diskurs übernimmt Kunstvermittlung die Aufgabe, die Funktionen von Ausstellungshäusern zu erweitern und als Orte gesellschaftlicher Veränderung zu begreifen. Hier gibt es auch Anknüpfungspunkte an eine kritische und politische Kulturarbeit, die sich als Einmischung in gesellschaftliche, politische und kulturelle Debatten versteht und auch konkrete Schritte setzen möchte, die in die Strukturen hineinwirken (vgl. Moser \& Gülcü, 2018).

PPB: Die kritischen und politischen Diversity-Ansätze sehen sich als antidiskriminatorisch und machtkritisch. Bei ihnen steht gesellschaftlicher Wandel hin zu sozialer Gerechtigkeit im Mittelpunkt (vgl. Adams et al., 2018; Eggers, 2011; Rosenstreich, 2011; Castro Varela \& Dhawan, 2011; Micossé-Aikins \& Sharifi, 2019). Das Kritische Diversity korreliert also mit Mörschs transforma-

10 Die documenta (https://www.documenta.de, [17.07.2020]) ist ein alle fünf Jahre stattfindendes, jeweils 100 Tage dauerndes Ausstellungsformat für zeitgenössische Kunst. Standort der documenta ist Kassel und erstmals wurde sie dort 1955 veranstaltet. Im Jahr 2007 fand die 12. Ausgabe der documenta statt. 
tivem Ansatz, dass eben, um ein Beispiel zu nennen, keine unhinterfragte weiße Hegemonie mehr möglich ist - mit all ihren Inhalten, Ein- und Ausschlussmechanismen -, dass Weiße nicht automatisch bessere Chancen haben. Da bezieht sich der Verein ][diskursiv etwa auf Ansätze des Kritischen Weißseins und der postkolonialen Theorien ${ }^{11}$.

AM: Auch die Kritische Kulturvermittlung, die sich u.a. auf Postcolonial und Queer Studies bezieht, verfolgt das Ziel, dass sich das ganze gesellschaftliche System verändert. Damit sich Institutionen und Kulturlandschaft ändern, braucht es jedoch konkrete Maßnahmen, die über Kulturvermittlung hinausgehen oder aus dieser heraus entstehen und sämtliche Aspekte der Kulturarbeit betreffen - insbesondere auch die organisatorische Ebene und die der Kulturpolitik. Mark Terkessidis (2017) spricht von sogenannten Vielheitsplänen, die in Bezug auf unsere Gesellschaft der Vielheit zu entwickeln seien, um einen Perspektivenwechsel und eine Neujustierung der Organisationen zu bewerkstelligen. Migration sieht er dabei als „eine Art Passepartout“ (Terkessidis, 2017, S. 9), um zahlreiche grundsätzliche Aspekte des Wandels zu diskutieren. Viele seiner allgemeinen Überlegungen lassen sich auf den Kunst- und Kulturbereich umlegen, etwa die Forderung nach umfassender Organisationsentwicklung, Anonymisierung von Bewerbungen, Erweiterung der Netzwerke, Änderung der Kommunikationskanäle, Thematisierung rassistischer Wissensbestände etc. Kulturarbeit als kritische Praxis ist als Prozess einer radikalen Neuausrichtung des gesamten Kulturbetriebs zu sehen (vgl. u. a. Aikins \& Gyamerah, 2016; Moser, 2019).

\section{Selbstbestimmung und Fremdbestimmung}

AM: Ich bin gedanklich noch bei dem Dilemma, das wir vorhin diskutierten, also dass man von Identitäten wegkommen müsse. Paul Mecheril (2012) spricht von der „Unmöglichkeit der Anerkennung“ und meint damit, dass im Grunde jede Anerkennung von Identitätsentwürfen immer auch mit einer Begrenzung einhergeht, also, dass nur einige Aspekte oder jemand aus einer spezifischen Perspektive er- und anerkannt wird. Dies hängt mit der grundsätzlichen Unmöglichkeit umfassender Anerkennung zusammen - oder wie Mecheril sagt: „Der Andere ist nicht anerkennbar, da der Andere nicht erkennbar ist." (Mecheril, 2012, S. 31) Daher braucht es eine Reflexion über dieses Phänomen sowie die Anerkennung der Nicht-Erkennbarkeit bzw. der Unbestimmtheit des oder der Anderen. Dieses paradoxe Moment müsste daher

11 Einen Einblick in die beiden Forschungsansätze geben u.v.a. die einführenden Texte von DiAngelo (2018), Eggers, Kilomba, Piesche \& Arndt (2005) sowie Castro Varela \& Dhawan (2005). 
immer auch ein Moment allgemeiner Bildung sein, was heißt, es immer mit zu bedenken. Beispielsweise wenn mit der Kategorie Menschen mit Behinderung gearbeitet wird, um festzustellen, von welchen Ausschlüssen die Personen betroffen sind. Natürlich werden mit der Verwendung solcher Kategorien diese auch wieder mit hergestellt und verfestigt. Aber geht es ganz ohne sie?

PPB: Es geht schon auch ohne Kategorisierung, zumindest ohne Fremdkategorisierung. Diese findet meist von Seiten der Organisationen und Personen in Kunstvermittlung und Kulturarbeit statt, wenn sie nicht selber Teil der LGBTIQA2 $+{ }^{12}$ - oder Be_Hinderten ${ }^{13}$ - oder BIPoC ${ }^{14}$-Communities sind und Menschen stereotypisieren. Es geht darum, danach $\mathrm{zu}$ fragen, wie z.B. Be_Hinderung hergestellt wird als etwas, das weniger wert sei als eine bestimme Vorstellung von Gesundheit (vgl. Magdlener, 2018): in unseren tagtäglichen Handlungen, über Gesetze, durch bauliche Stufen und Lautsprache, durch Stereotypen etc. In vielen Diversity-Ansätzen gilt die Gleichung: „In unserem Team haben wir eine Behinderte + einen Schwulen + einen Migranten + eine Frau - deshalb sind wir ein diverses Team." Kritische Diversity-Ansätze kritisieren diesen Zugang und fordern die Beschäftigung mit Diskriminierungsstrukturen und ihren intersektionalen Verwobenheiten.

AM: ... da bei vielen anderen Diversity-Ansätzen die Strukturen unangetastet bleiben.

PPB: Genau. Kunst- und Kultureinrichtungen sollten sich daher Fragen stellen wie: Was für Diskriminierungs-, Privilegierungs- und Normierungsprozesse finden statt? Dabei haben Sprache und Kommunikation einen zentralen Stellenwert. Diversity entsteht über sprachliche Handlungen - Vlatka Frketić nennt das in ihren Trainings Communicating Diversity. Die sprachliche Ebe-

12 Die Abkürzung LGBTIQA2+ steht für Lesbian, Gay, Bisexual, Trans, Inter, Queer, Asexual bzw. Agender, Two-Spirit und versucht, in kurzer Form verschiedene Aspekte und Identitäten sexueller und geschlechtlicher Vielfalt abzubilden. TwoSpirit (2) soll die nicht-westlichen, oftmals durch Kolonialismus aber auch durch Kirche/n verfolgten und teilweise zerstörten Aspekte und Identitäten sichtbar machen. Das Plus (+) am Ende zeigt an, dass die sexuelle und geschlechtliche Vielfalt weit über diese wenigen Begriffe und Konzepte hinausgeht.

13 Enthinderungsaktivist_innen nutzen immer häufiger den selbstbestimmten Begriff Be_Hinderung bzw. be_Hindert mit Unterstrich und großem H. Mit der queeren Sprachalternative des Unterstrichs _ soll symbolisch ein Raum aufgemacht werden für eine Vielzahl unterschiedlichster Menschen und Lebensformen, die meist pauschal und oft fremdbestimmt als die Behinderten bezeichnet oder beschimpft werden. Mit dem groß geschriebenen $\mathrm{H}$ wird deutlich gemacht, dass Menschen nicht behindert sind, sondern über äußere Umstände behindert werden. (Zum Unterstrich vgl. s_he 2003; zur Verwendung des Begriffes Be_Hinderung vgl. u.a. Payk, 2019)

14 BIPoC steht für Black People, Indigenous People and People of Color. Dies sind selbstbestimmte, aktivistische Begriffe, die einerseits rassistische Benennungen ersetzen und andererseits für Empowerment stehen. 
ne wird in Reflexionsprozessen oft übersehen, dabei sind Fragen wie: „Was mache ich, wenn ich spreche, höre, gebärde, lese?", „Welche unausgesprochenen Regeln gibt es in der jeweiligen Organisation (Gesten, Kleidung, Ein- oder Mehrsprachigkeit, Fachsprachen etc.)?“ oder „Welchen Stellenwert haben die verschiedenen Nationalsprachen in der Gesellschaft?" Dies sind wichtige und zentrale Fragen, um Ein- und Ausschlussprozesse zu entlarven.

AM: Die Selbstreflexion der eigenen Institution müsste also im Kontext einer kritischen Kulturarbeit ganz oben stehen. Auf Basis dieser wären dann in einer Art freiwilligen Selbstverpflichtung konkrete weitere Schritte zu setzen. Terkessidis plädiert in Bezug auf Institutionen allgemein für ein der jeweiligen Organisation angemessenes, internes Benchmarking, das die Setzung strategischer Ziele mit der Entwicklung geeigneter Evaluationsmethoden verbindet (vgl. Terkessidis, 2017, S. 51). Wichtig wäre meiner Meinung nach, dass es für (selbst-)kritische Nachdenk- und Neuausrichtungsprozesse von Kultureinrichtungen auch entsprechende kulturpolitische Rahmenbedingungen und Anreize gibt, etwa spezifische Förderungen oder anderweitige Unterstützung.

Ich überlege gerade in Bezug auf die vier oben erwähnten Diskurse der Kulturvermittlung, woran sich Transformationen festmachen lassen. Dabei fällt mir ein: Im Schloss Ambras in Innsbruck ist Das Bildnis eines behinderten Mannes aus dem 16. Jahrhundert zu sehen. Lange war darüber nichts bekannt bis Volker Schönwiese dazu ein interdisziplinäres Forschungsprojekt durchgeführt und damit auch die verdrängte Geschichte publik gemacht hat (vgl. Schönwiese \& Mürner, 2005). Das Bild hat in der offiziellen Sammlung einen Platz bekommen und kann nun besichtigt werden, zwar im Rahmen der Kunst- und Wunderkammer, aber zumindest kontextualisiert. Das Bild ist damit Teil der offiziellen Geschichte geworden, jener Geschichte, die in den Führungen erzählt wird, das heißt, es ist mittlerweile ein Teil des reproduktiven Diskurses.

PPB: Für mich wäre das reproduzierend auf der individuellen und der interaktionalen Ebene, aber nicht auf der gesellschaftlichen, weil Be_Hinderung sonst nicht in der hohen Kunst ausgestellt wird, außer als etwas Negatives (vgl. Akarçeşme, Baumgartinger \& Egermann, 2018).

AM: Stimmt. Es wäre spannend, die verschiedenen Ebenen von Diversity mit den unterschiedlichen Diskursen der Kulturvermittlung in Verbindung zu bringen, also diese Diskurse in Hinblick auf die dabei tangierten Diversity-Ebenen zu analysieren. Das ermöglicht eine differenziertere Auseinandersetzung.

PPB: Ja, und um der Differenziertheit besser gerecht zu werden, arbeitet das Kritische Diversity zusätzlich mit verschiedenen Dimensionen, etwa der sprachlichen, historischen, emotionalen, und achtet insbesondere auf deren Verwobenheiten. Weiters werden Diskriminierungsfelder unterschieden, wie 
etwa Rassismus, Antisemitismus, Ableismus, Transfeindlichkeit, Homophobie, und normative Strukturen, wie etwa Gesundheitsnormen, Heteronormativität, christliche Hegemonie. In der Reflexion werden alle Ebenen, Dimensionen und Felder genau angeschaut. ${ }^{15}$ Vielleicht funktioniert dann - mit diesem multidimensionalen Blick - auch das Dilemma der Anerkennung anders. Mit dieser Differenzierung werden pauschale Aussagen vermieden und es wird klar, dass wir innerhalb einer historisch gewachsenen, hierarchisch strukturierten Gesellschaftsordnung unterschiedlich positioniert sind, was manchen Menschen mehr und anderen weniger Teilhabe erlaubt - gegenüber manchen Diversity-Ansätzen sind wir eben nicht alle gleich und haben auch nicht die gleichen Chancen.

Damit beschäftigen sich die Trans Studies auch stark: Einerseits verstehen viele Menschen Trans als Identität; gleichzeitig werden die Diskriminierungsstrukturen rund um das, was als Trans gilt, dekonstruiert. Du hast einerseits die Materialität des Körpers, dem Identität zugeschrieben wird. Aber es gibt eben auch die Selbstidentität und im Grunde ist das vielleicht auch gar kein Dilemma, es ist einfach nur eine Gleichzeitigkeit. Es ist eine Gleichzeitigkeit von Selbstdefinition und den gesellschaftlichen Normen bzw. Strukturen (z. B. Gesetze), anhand dessen wiederum das Kritische Diversity seinen Diskriminierungsbegriff hauptsächlich festmacht. Bei den Trans Studies gibt es diese Gleichzeitigkeit der Anerkennung selbstbestimmter Identitäten - z. B. transsexuell, transgender, trans ${ }^{\star}$, trans_, nonbinary ..., aber auch (trans) Mann und (trans) Frau - und der Dekonstruktion von Geschlecht und Geschlechterbinarität, das Herausarbeiten der Verbindungen von Geschlecht und Posthumanismus (dass der Mensch sich historisch über die anderen Wesen erhöht hat), Dekolonisierung etc.

AM: Meinst du damit eine Selbstverortung im Sinne von Gayatri Spivaks Konzept des Strategischen Essenzialismus ${ }^{16}$ ? Der Verein maiz beispielsweise arbeitet mit der Selbstbezeichnung Migrant( $\left.{ }^{\star} i n n\right) e n$. Diese wird als Gegenentwurf verwendet, also als Benennung eines oppositionellen Standorts und als Bestimmung der eigenen politischen Identität (vgl. Salgado \& maiz, 2015,

15 Dazu haben die Studierenden meiner Lehrveranstaltung Diversity in Kunst und Kultur am Kooperationsschwerpunkt Wissenschaft und Kunst ein Modell erstellt, das alle diese Dimensionen mitdenkt und von Kunst- und Kultureinrichtungen angewendet werden kann (vgl. Bründl, Jacobs, Schatz \& Simair, 2018).

16 Ausgehend davon, dass Kategorien für Zugehörigkeiten und deren Repräsentationen Konstruktionen sind, die immer auch Ausschlüsse produzieren, plädiert die postkoloniale Theoretikerin Gayatri Chakravorty Spivak dafür, Zugehörigkeiten trotzdem nicht aufzugeben, sondern sich strategisch bestimmten Gruppen zuzuordnen. Dadurch können Unterdrückungsverhältnisse benannt, der Vereinzelung entgegengewirkt und politisches Gewicht erlangt werden (vgl. u. a. Spivak, 2008). 
S. 37-38). Durch die Setzung einer strategischen Identität bezieht man sich auf eine Kategorie und dekonstruiert diese gleichzeitig.

PPB: Ja, so wie der Begriff Schwarz, groß geschrieben, und der Begriff weiß, klein und kursiv geschrieben, von Schwarzen und People of Color als politische Begriffe und nicht als Identitätsbegriffe eingeführt wurden (vgl. Eggers et al., 2005). Es geht dabei vor allem darum, einerseits einen positiven, selbstbestimmten Begriff zu finden (Schwarz) und andererseits auch die implizite Norm (weiße Vorherrschaft) überhaupt mal zu benennen. So wie der Begriff Cis- entwickelt wurde, um die Norm der nicht-trans Personen zu benennen (vgl. Baumgartinger, 2017, Kapitel 2).

AM: Es gibt diese Gleichzeitigkeit von Selbstverortung und Fremdbestimmung. Die Selbstdefinition bzw. -verortung als Strategie ist wichtig, um politisch handlungsfähig zu sein. Problematisch ist die Festschreibung von außen, wie sie auch in der Kulturvermittlung häufig vorkommt, wenn zum Beispiel Programme für Personen mit Migrationshintergrund angeboten werden. Die politisch-strategische Dimension von Selbstbezeichnungen lässt sich in der Kommunikation nach außen oft schwer mittransportieren. Dann wird ein Begriff sehr verkürzt aufgenommen und man wird auf Kategorien reduziert, gegen die man sich eigentlich wendet. Das hängt aber auch damit zusammen, dass die Expertise der Angehörigen von Selbstorganisationen und Netzwerken, in denen selbstermächtigende Begriffe und Konzepte entwickelt und theoretisiert werden, bei der Umsetzung von Kunst- und Kulturprojekten oftmals keine Berücksichtigung findet.

PPB: Würden wir zum Beispiel die vielen Kunst- und Kulturprojekte mit Geflüchteten mit Modellen des Kritischen Diversity analysieren, würden mehrere Diskriminierungsformen sichtbar: Rassismus, oft Klassismus - aber auch Adultismus, also wenn Jugendliche temporär in eine Produktion einbezogen werden, aber keine oder keine angemessene Bezahlung für ihre Kulturarbeit bekommen und, weil sie zum Beispiel nicht genannt werden oder keine weitere Einbindung in die Kultureinrichtung oder andere Projekte stattfindet, keinen längerfristigen Nutzen davon haben. Wenn sie jedoch z. B. ein Zertifikat bekämen, das sie bei einer Bewerbung vorzeigen könnten, oder wenn die Kultureinrichtung sie als Kulturvermittelnde anstellt - das wäre der Beginn einer Transformation. Es geht bei transformativen Kulturprojekten also auch um Umverteilung und darum, den sozialen Status, den so eine Einrichtung hat, zu nutzen, damit andere mehr Chancen haben auf gesellschaftliche Teilhabe und Teilnahme als bisher.

AM: Genau. Man kommt immer wieder auf ähnliche Punkte und Fragen. Auch im universitären Kontext sollten diese kritisch beleuchtet werden. Es ist zu fragen, wo - in den Personalstrukturen, Lehr- und Forschungsinhalten etc. - Diskriminierungen stattfinden und Privilegien unhinterfragt fortgesetzt wer- 
den. Beispielsweise sind die Leitungsebenen nach wie vor mehrheitlich von weißen Männern besetzt.

\section{Kulturarbeit als (un-)kritische Praxis?}

PPB: Ein Diversity-Prozess ist keine Wohlfühlveranstaltung, auch wenn es oft so verstanden wird, sondern kann anstrengend sein, er bedeutet frictions and fractions (vgl. Stryker, Currah \& Moore, 2008): Da reibt es sich, da sind Auseinandersetzungen, das ist Diversity. In diesem Sinne geht es auch beim transformativen Diskurs - wenn er wirklich ernst genommen und umgesetzt wird - ans Eingemachte. Denn wenn eine Person eingestellt wird, die z. B. aufgrund bestimmter körperlicher Gegebenheiten oder sogenannter Lernschwierigkeiten langsamer ist, hat das Auswirkungen auf einen Betrieb, beispielsweise veränderte Arbeitsabläufe, anderen Rhythmen und Zeitlichkeiten als gewohnt. Das sind Themen, über die ungern gesprochen wird. Dabei hat das überhaupt nichts mit der Person zu tun, sondern mit der organisationalen und gesellschaftlichen Ebene. Was bedeutet Leistung? Wie schnell müssen Menschen funktionieren? Welche Vorstellung von Zeit wird als Norm vorausgesetzt? Da kommen Ableismus ${ }^{17}$ und Kapitalismuskritik dazu. Deshalb ist beim Kritischen Diversity eine intersektionale Herangehensweise, die einzelne Normen, Werte und Mächte von z.B. Kultureinrichtungen zusammendenkt, wichtig, denn Diskriminierungs- und Ausschlussstrukturen funktionieren insbesondere, weil mehrere Machtebenen miteinander verwoben sind.

AM: Kapitalistische Interessen und Logiken sind auch in Kultureinrichtungen kritisch zu sehen. Gerade bei Kultur- und Kunstvermittlungsangeboten stehen oft wirtschaftliche Interessen dahinter: Beispielsweise stellen Verantwortliche von Museen fest, dass ihr Publikum durchschnittlich 60+ Jahre alt ist und dass sie, wenn sie sich nichts Grundlegendes überlegen, in den nächsten Jahren zusperren müssen. Es geht also auch um Existenzsicherung und die Legitimation der eigenen Einrichtungen. Audience-Development-Konzepte sind

17 Der Begriff Ableismus bezeichnet „jene Form der Diskriminierung, von der Personen mit Behinderung* aufgrund dieser Zuschreibung betroffen sind. Der Begriff wird vom englischen ,to be able (Deutsch: ,fähig sein') abgeleitet. Dieser Begriff meint, dass unser menschlicher Körper sowie unser Denken, Fühlen und Handeln auf eine vom gesellschaftlichen System vorgegebene Weise funktionieren müssen, und falls sie dies nicht tun, zumindest kontrollierbar sein müssen. Diese Vorstellungen vom Fähig-Sein tragen jene Gewalt in sich, die als Ableismus bezeichnet wird. Nicht-Behinderung*, also angebliche Fähigkeit, wird dabei als Standard vorausgesetzt. Das ,Funktionieren' wird in einer Art Ideologie einer physischen, psychischen oder mentalen Behinderung* gegenüber gestellt und als ,etwas Besseres' gewertet (vgl. Hutson 2010: 61 f.)." (Magdlener, 2018, o. S.) 
u. a. auch aus der Frage entstanden „Wie kann ich, als Kulturinstitution, ein größeres, vielfältigeres, zahlendes Publikum gewinnen?“ - und weniger aufgrund der Überlegung „Wie kann eine breite kulturelle Teilhabe gewährleistet werden?"

Ein gutes Beispiel für die Veränderung von Institutionen ist das Berliner Gorki-Theater. Dort spiegelt sich gesellschaftliche „Vielheit“"18 auf unterschiedlichen Ebenen wider, ebenso Aspekte einer kritischen und politischen Kulturarbeit. Etwa sind auf der Personalebene Personen verschiedener Herkünfte, sozialer Schichten, sexueller Orientierung etc. anzutreffen, was Auswirkungen auf alle anderen Ebenen hat: Die Aufführungen und Projekte sind mehrsprachig, das Publikum ist heterogener, das Programm beinhaltet Stücke von Autor $\left({ }^{*}\right.$ inn)en aus verschiedenen Kulturen und Zusammenhängen, nicht kanonisierte und kanonisierte Texte - wobei zum Beispiel der deutsche Kanon kontinuierlich dekonstruiert und aus neuen Perspektiven angeeignet wird. Das Gorki-Theater ist eine Art Vorzeigeprojekt, auf das oft verwiesen wird. Es gibt auch noch andere Einrichtungen und Projekte, in Wien zum Beispiel die Brunnenpassage (vgl. Pilić \& Wiederhold, 2015).

PPB: Ja, die Brunnenpassage ist auf alle Fälle ein offener Raum. Zum Beispiel wollte sich der Verein ][diskursiv dort vor einigen Jahren für den International Transgender Day of Remembrance ${ }^{19}$ einmieten. Die Brunnenpassage war begeistert, sie hatten leider keinen Platz an dem Tag, aber dass die Verantwortlichen so offen reagiert haben, war damals etwas Besonderes.

AM: Im Gegensatz zu vielen Theaterstücken und Projekten mit Geflüchteten merkt man beim Gorki-Theater, wie wichtig dort der Anspruch an Professionalität ist, das heißt, Schauspielende treten als solche auf die Bühne, sie verkörpern Rollen und nicht eigene Schicksale. Es gibt im Kunst- und Kulturbereich viele paternalistische Projekte, wo gemeinsam etwas gemacht wird, um die Zeit bis zur Entscheidung über den Aufenthaltsstatus zu überbrücken, wodurch Menschen letztlich aber auch in einem prekären Status gehalten werden. Gerade im Zusammenhang von Kulturvermittlungsprojekten mit Geflohenen

18 In der Verwendung des Begriffs beziehe ich mich auf Mark Terkessidis, der mit dieser Bezeichnung unterstreichen möchte, „dass es keine Einheit mehr geben wird, nicht mehr geben kann" (Terkessidis, 2017, S. 17), auch wenn die Vorstellung von Einheit in einem Nationalstaat immer eine große Bedeutung hatte. Für das $\mathrm{Zu}$ sammenleben in einer Gesellschaft ist allerdings eine gewisse Basis von Einigkeit notwendig. Grundlage für die Gestaltung von Einigkeit ist die Akzeptanz von Vielheit (vgl. Terkessidis, 2017, S. 17-18).

19 Am 20. November jeden Jahres wird in weltweiten Aktionen der ermordeten trans Personen und ihren Verbündeten gedacht (verfügbar unter: https://tdor.info [17.07.2020]). Der Verein ][diskursiv organisierte diesen Gedenktag ab 2006 für mehrere Jahre in Wien. 
ist Politisierungsarbeit ${ }^{20}$ enorm wichtig, um die Handlungsmacht, Expertise und rechtliche Situation Geflüchteter zu stärken, aber auch um bei der so genannten Mehrheitsgesellschaft kritische Reflexionen - u.a. dahingehend, wer von welcher Hilfe wie profitiert - in Gang zu setzen und gemeinsam Perspektiven zu entwickeln. ${ }^{21}$

Häufig finden in Museumskontexten Vermittlungsprojekte - zum Beispiel im Kontext von Flucht - statt, deren Ergebnisse (z. B. Ausstellungen etc.) durchaus interessant sind. Wenn man sich dann aber den Entstehungsprozess genauer ansieht, stellt man fest, dass mit sehr traditionellen Kulturbegriffen gearbeitet wurde, die Zusammenarbeiten hierarchisch waren, den Mitwirkenden keine Honorare bezahlt wurden und die Menschen wieder nur auf ihre Fluchtgeschichte oder Identität aufgrund ihrer Herkunft reduziert wurden und also asymmetrische Verhältnisse reproduziert wurden. Wichtig ist daher, dass das Wissen, das in diskriminierungskritischen Projekten wie denen der Brunnenpassage oder auch der WIENWOCHE ${ }^{22}$ generiert wird, in Institutionen einfließt. Eine ernst gemeinte Diversitätspolitik hieße, „dass alle diese Menschen in anderen Institutionen landen, weil man sie da händeringend sucht" (Moser \& Gülcü, 2018).

PPB: Das erinnert mich an die Forderung der Enthinderungsbewegung aus den 1990er-Jahren: „Nothing about us without us!“ (Charlton, 1998, S. 3) Sie ist eine aktivistische Reaktion auf Pathologisierung, Fremdbestimmung und auch solche Ausnutzungssysteme im Kunst- und Kulturbereich sowie in der Wissenschaft. Der Slogan wird zurückgeführt auf Michael Masutha und William Rowland, zwei Personen des Enthinderungsaktivismus Südafrikas, die sich ihrerseits auf eine unbekannte Person des Enthinderungsaktivismus in Osteuropa bezogen (vgl. Charlton, 1998, S. 3).

AM: Diese Forderung ist auch bei der australischen Selbstorganisation RISE - Refugees, Survivors and Ex-Detainees zentral. 2015 formulierten die Mitwirkenden zehn Punkte, die in der künstlerischen Zusammenarbeit Nicht-

20 Diese kann dadurch geleistet werden, dass Geflüchtete z. B. Zugang zu rechtlichen Informationen, politischen Vertretungen, Netzwerken, Infrastrukturen etc. erhalten und ihre Handlungsmacht in Bezug auf die prekäre Lage stärken können.

21 Wie beispielsweise auf dem Berliner Kongress Zivilgesellschaft 4.0 - Geflüchtete und digitale Selbstorganisation, wo Expert( ${ }^{*}$ inn)en, Aktivist(*inn)en und Unterstützer*innen gemeinsam Handlungsempfehlungen als Petition an den Bundestag entwickelten. Verfügbar unter: https://www.hkw.de/de/programm/projekte/2016/ civil_society_4_0/deklaration_civil_society/zivilgesellschaft_11_handlungsempfeh lungen.php [17.07.2020].

22 WIENWOCHE (http://www.wienwoche.org [17.07.2020]) ist ein seit 2012 jährlich im September zu verschiedenen Ausschreibungsthemen stattfindendes Kulturfestival in Wien. Es experimentiert mit der Verschmelzung von kreativen Praktiken und Aktivismus und versteht Kulturarbeit als ein Einmischen in gesellschaftliche, politische und kulturelle Debatten. 
Geflohener/Nicht-Asylsuchender mit Geflohenen und Asylsuchenden zu beachten seien (vgl. Canas, 2015). Diese wurden im Zuge einer kritischen Auseinandersetzung mit Projekten, die im Kontext des Sommers der Migration 2015 entstanden sind, oft zitiert. Es wird gefordert, dass Kunstschaffende die eigene Intention kritisch hinterfragen, eigene Privilegien realisieren, im Vorfeld über die Community und bereits durchgeführte Projekte recherchieren, sich bewusst sind, dass Kunst nie neutral ist und dass in der Arbeit der Fokus auf den Prozess anstatt auf das Ziel gelegt wird.

PPB: Für kritische Kulturarbeit ist es vor allem wichtig, einen Schritt zur Seite zu gehen und zu erkennen, dass es bereits sehr viel selbstbestimmtes Wissen, Strategien und Forderungen zu sogenannten Minderheitenthemen gibt. Sie sind schon lange da, bevor die Wissenschafts-, Kunst- und Kulturbereiche auf sie aufmerksam werden. Denn marginalisierte Gruppen müssen viel und hart arbeiten, bevor sie überhaupt vom Mainstream gehört werden. Und Wissenschaft, Kunst und Kultur sind elitäre Bereiche, die lange brauchen, bis sie aufmerksam werden. Es gibt sehr viel Forschung und Kulturproduktion von und mit trans_, inter*, queeren Personen mit sogenanntem Migrationshintergrund, Geflüchteten, Be_Hinderten, Roma und Sinti etc., die von kritischen Diversity- und Kulturarbeitsansätzen, aber auch machtvollen Institutionen wie Universitäten und (Staats-)Museen ernst genommen werden müssten. Auch an konkreten Handlungsanleitungen mangelt es nicht - es hält sich nur selten jemand dran.

\section{Literatur}

Adams, M., Blumenfeld, W. J., Catalano, D. C. J., Dejong, K., Hackman, H. W., Hopkins, L. E., Love, B., Peters, M. L., Shlasko, D. \& Zuniga, X. (Hrsg.). (2018). Readings for Diversity and Social Justice. New York: Routledge.

Ahmed, S. (2011). "You end up doing the document rather than doing the doing“. Diversity, Race Equality und Dokumentationspolitiken. In M. Castro Varela \& N. Dhawan (Hrsg.), Soziale (Un)Gerechtigkeit. Kritische Perspektiven auf Diversity, Intersektionalität und Antidiskriminierung (S. 118-137). Berlin: LIT Verlag. Ahmed, S. (2012). On Being Included: Racism and Diversity in Institutional Life. Durham: Duke University Press. https://doi.org/10.1215/9780822395324

Aikins, J. K. \& Gyamerah, D. (2016). Handlungsoptionen zur Diversifizierung des Berliner Kultursektors. Verfügbar unter: https://vielfaltentscheidet.de/wp-con tent/uploads/2017/04/Final-für-Webseite_klein.pdf [17.07.2020].

Akarçeşme, D., Baumgartinger, P. P. \& Egermann, E. (2018). Wer hat die Deutungshoheit, die Herrschaftsmacht und die Sprech*position, um Kultur zu schaffen oder sie zu demokratisieren? Eva Egermann im Interview mit Dilara Akarçeşme und Persson Perry Baumgartinger. p/art/icipate - Kultur aktiv 
gestalten \#9. Verfügbar unter: https://www.p-art-icipate.net/wer-hat-die-deu tungshoheit-die-herrschaftsmacht-und-die-sprechposition-um-kultur-zu-schaf fen-oder-sie-zu-demokratisieren/ [17.07.2020].

Bargehr, G. \& Johnston-Arthur, A. E. (2018). Multiperspektivischer Reflexionsansatz. Handout des Workshops „Machtkritisches Handeln und Weiß-sein“, 15. -16. Juni 2018. Wien: Universität Wien.

Baumgartinger, P. P. (2017). Trans Studies. Historische, begriffliche und aktivistische Aspekte. Wien: Zaglossus.

Baumgartinger, P. P. \& Frketić, V. (2019). Kritisches Diversity und Kulturarbeit: Wenn Aktivismus und Erfahrungswissen in den Mittelpunkt gerückt werden. In E. Zobl, E. Klaus, A. Moser \& P. P. Baumgartinger, Kultur produzieren. Kritische kulturelle Produktion und künstlerische Praxen (S. 195-211). Bielefeld: transcript. https://doi.org/10.14361/9783839447376-013

Bründl, A., Jacobs, S., Schatz, R. \& Simair, C. (2018). Diversity Balloons - Ein Diversity-Modell für Kunst- und Kulturinstitutionen. p/art/icipate - Kultur aktiv gestalten \#9. Verfügbar unter: https://www.p-art-icipate.net/diversity-balloonsein-diversity-modell-fuer-kunst-und-kulturinstitutionen/ [17.07.2020].

Canas, T. (2015). 10 things you need to consider if you are an artist - not of the refugee and asylum seeker community - looking to work with our community. RISE. Verfügbar unter: http://riserefugee.org/10-things-you-need-to-considerif-you-are-an-artist-not-of-the-refugee-and-asylum-seeker-community-look ing-to-work-with-our-community/ [17.07.2020].

Castro Varela, M. \& Dhawan, N. (2005). Postkoloniale Theorie. Eine kritische Einführung. Bielefeld: transcript. https://doi.org/10.14361/9783839403372

Castro Varela, M. \& Dhawan, N. (Hrsg.). (2011). Soziale (Un)Gerechtigkeit. Kritische Perspektiven auf Diversity, Intersektionalität und Antidiskriminierung. Berlin: LIT-Verlag.

Charlton, J. I. (1998). Nothing About us Without Us. Disability Oppression and Empowerment. Berkeley: University of California Press. https://doi.org/10. $1525 / 9780520925441$

DiAngelo, R. (2018). White Fragility. Why It's So Hard for White People to Talk About Racism. Random House LCC US.

Eggers, M. M., (2011). Diversity / Diversität. In S. Arndt \& N. Ofuatey-Alazard (Hrsg.), Wie Rassismus aus Wörtern spricht (S. 256-263). Münster: Unrast.

Eggers, M. M., Kilomba, G., Piesche, P. \& Arndt, S. (2005). Mythen, Masken und Subjekte. Kritische Weißseinsforschung in Deutschland. Münster: Unrast.

Frketić, V. (2014). Mehrsprachigkeit und Diversität. Ein Handbuch aus der Arbeitspraxis von Migrantinnenorganisationen. (Hg. v. LEFÖ Wien.) Verfügbar unter: https://mehrsprachen.wordpress.com/mehrsprachigkeit-diversitat-ein-hand buch/ [17.07.2020].

Kendall, G. (2007). What Is Critical Discourse Analysis? Ruth Wodak in Conversation with Gavin Kendall [38 Absätze]. Forum Qualitative Sozialforschung/ 
Forum Qualitative Research, 8 (2). Verfügbar unter: http://www.qualitativeresearch.net/index.php/fqs/article/view/255/561 [17.07.2020].

Magdlener, E. (2018). Über Körper, kulturelle Normierung und die Anforderung einer „Kultur für alle“ im Kontext von Dis_ability. p/art/icipate - Kultur aktiv gestalten \#9. Verfügbar unter: https://www.p-art-icipate.net/ueber-koerperkulturelle-normierung-und-die-anforderung-einer-kultur-fuer-alle-im-kontextvon-dis_ability/ [17.07.2020].

Mandel, B. (Hrsg.). (2005). Kulturvermittlung zwischen kultureller Bildung und Kulturmarketing. Eine Profession mit Zukunft. Bielefeld: transcript. https://doi.org/ $10.14361 / 9783839403990$

Mecheril, P. (2012). Ästhetische Bildung. Migrationspädagogische Anmerkungen. In Institut für Auslandsbeziehungen (ifa), Institute for Art Education (IAE), Zürcher Hochschule der Künste ZHdK \& Institut für Kunst im Kontext der Universität der Künste Berlin (Hrsg.), Kunstvermittlung in der Migrationsgesellschaft/Reflexionen einer Arbeitstagung - 2011 (S. 26-35). Verfügbar unter: https://nbn-resolving.org/urn:nbn:de:0168-ssoar-54733-2 [17.07.2020].

Micossé-Aikins, S. \& Sharifi, B. (2019). Kulturinstitutionen ohne Grenzen? Annäherung an einen diskriminierungskritischen Kulturbereich. KULTURELLE BILDUNG ONLINE. Verfügbar unter: https://www.kubi-online.de/artikel/ kulturinstitutionen-ohne-grenzen-annaeherung-einen-diskriminierungskriti schen-kulturbereich [17.07.2020].

Moser, A. (2015). Freie Kunst- und Kulturproduktion. Ein Überblick. In S. Lang (Hrsg.), Kunst, Kultur - und Unternehmertum?! Aspekte, Widersprüche, Perspektiven. Eine österreichische Studie (S. 32-44). Wien: mandelbaum wissenschaft.

Moser, A. (2019). Kulturarbeit in der ,Migrationsgesellschaft'. Ungleichheiten im Kulturbetrieb und Ansatzpunkte für eine kritische Neuausrichtung. In E. Zobl, E. Klaus, A. Moser \& P. P. Baumgartinger, Kultur produzieren. Kritische kulturelle Produktion und künstlerische Praxen (S. 117-133). Bielefeld: transcript.

Moser, A. \& Gülcü, C. (2018). Radikalität findet dort statt, wo ich meine eigenen Regeln breche. Can Gülcü im Gespräch über politische Kulturarbeit und Grenzüberschreitungen in einer von Ungleichheit geprägten Gesellschaft. p/art/icipate - Kultur aktiv gestalten \#9. Verfügbar unter: https://www.p-articipate.net/radikalitaet-findet-dort-statt-wo-ich-meine-eigenen-regeln-breche [17.07.2020]. https://doi.org/10.14361/9783839441268-010

Mörsch, C. (2009). Am Kreuzungspunkt von vier Diskursen: Die documenta 12 Vermittlung zwischen Affirmation, Reproduktion, Dekonstruktion und Transformation. In C. Mörsch \& Forschungsteam der documenta 12 Vermittlung (Hrsg.), Kunstvermittlung II. Zwischen kritischer Praxis und Dienstleistung auf der documenta 12. Ergebnisse eines Forschungsprojekts (S. 9-33). Zürich: diaphanes.

Mörsch, C. \& Forschungsteam der documenta 12 Vermittlung (Hrsg.). (2009). Kunstvermittlung II. Zwischen kritischer Praxis und Dienstleistung auf der documenta 12. Ergebnisse eines Forschungsprojekts. Zürich: diaphanes. 
Mörsch, C., Schade, S. \& Vögele, S. (Hrsg.). (2018). Kunstvermittlung zeigen. Über die Repräsentation pädagogischer Arbeit im Kunstfeld. Representing Art Education. On the Representation of Pedagocigal Work in the Art Field. Wien: Zaglossus.

Payk, Katharina (2019). Hä? Was bedeutet be_hindert? Unser Glossar gegen die Panik von Wörtern. Diesmal: be_hindert. Missy Magazin, 12.03.2019. Verfügbar unter https://missy-magazine.de/blog/2019/03/12/hae-was-bedeutet-be_hin dert/ [17.07.2020].

Pilić, I. \& Wiederhold, A. (2015). Kunstpraxis in der Migrationsgesellschaft - Transkulturelle Handlungsstrategien am Beispiel der Brunnenpassage Wien. Art Practices in the Migrant Society -Transcultural Strategies in Action at Brunnenpassage in Vienna. Bielefeld: transcript. https://doi.org/10.14361/9783839431917

Rosenstreich, G. (2011). Antidiskriminierung und/als/trotz .... Diversity Training. In M. Castro Varela \& N. Dhawan (Hrsg.), Soziale (Un)Gerechtigkeit. Kritische Perspektiven auf Diversity, Intersektionalität und Antidiskriminierung (S. 230244). Berlin: LIT-Verlag.

s_he [Herrmann, S. K.] (2003). Performing the Gap: Anmerkungen zu einer radikalen Theorie der sexuellen Politik. arranca! linke Zeitschrift, 28/2003, 22-26. Verfügbar unter https://web.archive.org/web/20100117203646/http://arranca. nadir.org/arranca/article.do?id=245 [17.07.2020].

Salgado, R. \& maiz (2015). Aus der Praxis im Dissens. Wien, Linz, Berlin, London, Zürich: transversal texts.

Sato, H. (2009). Performing Essentialismus auf der documenta 12. In C. Mörsch \& Forschungsteam der documenta 12 Vermittlung (Hrsg.), Kunstvermittlung II. Zwischen kritischer Praxis und Dienstleistung auf der documenta 12. Ergebnisse eines Forschungsprojekts (S. 67-78). Zürich: diaphanes.

Schönefeld, D. (2017). Arbeiten und Unterscheiden. Zur Praxis des Diversity-Managements. Weinheim: Beltz Juventa.

Schönwiese, V. \& Mürner, C. (2005). Das Bildnis eines behinderten Mannes. Kulturgeschichtliche Studie zu Behinderung und ihrer Aktualität. Mit Beiträgen von Margot Rauch und Andreas Zieger. Psychologie und Gesellschaftskritik, 113, 95-125.

Sigusch, V. (2013). Sexualitäten: Eine kritische Theorie in 99 Fragmenten. Frankfurt am Main, New York: Campus Verlag.

Spivak, G. C. (2008). Can the Subaltern Speak? Postkolonialität und subalterne Artikulation. Mit einer Einleitung von Hito Steyerl. Wien: Turia + Kant.

Stryker, S., Currah, P. \& Moore, L. J. (2008). Introduction. Trans-, Women Studies Quarterly, 36 (3-4), 11-22. https://doi.org/10.1353/wsq.0.0112

Terkessidis, M. (2017). Nach der Flucht. Neue Ideen für die Einwanderungsgesellschaft. Ditzingen, Stuttgart: Reclam. 
Renate Reitinger

\section{Chancengleichheit als Entwicklungsaufgabe an Musikhochschulen}

\section{Bedingungen - Strukturen - Möglichkeiten}

\section{Einführung}

An Musikhochschulen als Ausbildungsinstitutionen für Musikberufe steht traditionell die hochspezialisierte Spitzenförderung im Mittelpunkt des Interesses, sodass dort die typischen Inklusionsthemen allgemeinbildender Schulen und Musikschulen (etwa ein äußerst heterogenes Leistungs- und Motivationsspektrum) bisweilen in den Hintergrund treten. Gleichzeitig sind gerade auch die Hochschulen für Musik u.a. aufgrund ihrer internationalen Ausrichtung Orte höchster Diversität: Kulturkreisspezifische Themen mischen sich hier wie überall in der Gesellschaft beispielsweise mit Unterschieden hinsichtlich genderspezifischer Identität, physischer und psychischer Verfassung sowie Begabungsdispositionen und sozioökonomischen Faktoren und betreffen sowohl die an den Hochschulen tätigen Studierenden und Lehrenden selbst als auch die Ausbildungsinhalte. Dies gilt insbesondere im Bereich der künstlerischpädagogischen Studiengänge. An Musikhochschulen besteht also die doppelte Herausforderung, zum einen als Institution mit Diversität angemessen umzugehen und zum anderen, durch die Ausbildung junger Menschen selbst gesellschaftsrelevante Haltungen und Veränderungen anzustoßen. Ein Ansatzpunkt hierfür ist der vorbildhafte Umgang mit Diversität seitens der Institution und der sie mit Leben füllenden Menschen. Im Folgenden sollen deshalb mögliche Ansatzpunkte und Bedingungen vorgestellt sowie die Chancen erläutert werden, die Musikhochschulen als Orte der Vielfalt innerhalb der Gesellschaft nutzen können. Besondere Bedeutung kommt dabei der jeweiligen Führungskultur zu. Von ihr bzw. den handelnden Funktionsträger $\left({ }^{*}\right.$ inne)n hängt das Gelingen von Diversity-Management und Inklusion in hohem Maße ab. Nur wenn hier das Thema Inklusion als strategische Entwicklungsaufgabe begriffen wird, kann ein Umdenken der gesamten Institution gelingen. 


\section{2. (Rahmen-)Bedingungen}

Basis und Rahmen aller Überlegungen bilden die politischen Gegebenheiten, im Folgenden wird beispielhaft auf die Bedingungen in der Bundesrepublik Deutschland Bezug genommen. Gesetzliche Grundlagen stellen sowohl das Grundgesetz (GG) selbst mit dem Diskriminierungsverbot (vgl. Art. 3 GG), als auch das Allgemeine Gleichbehandlungsgesetz (AGG), die Behindertengleichstellungsgesetze des Bundes und der Länder sowie die Ratifizierung der UN-Behindertenrechtskonvention im Jahr 2009 dar. Hierin verpflichten sich alle Vertragsstaaten, u. a. den diskriminierungsfreien und chancengleichen Zugang zur allgemeinen Hochschulbildung und zu lebenslangem Lernen zu sichern (vgl. Art. 24 Abs. 5 Behindertenrechtskonvention). Vor diesem Hintergrund wurden das Hochschulrahmengesetz (HRG) sowie die Hochschulgesetze der Länder entsprechend weiterentwickelt und Hochschulen müssen etwa gewährleisten, „dass behinderte Studierende in ihrem Studium nicht benachteiligt werden und die Angebote der Hochschule möglichst ohne fremde Hilfe in Anspruch nehmen können" ( $\$ 2$ Abs. 4 HRG). Ebenso wird darauf verwiesen, dass Prüfungsordnungen „die besonderen Belange behinderter Studierender zur Wahrung der Chancengleichheit berücksichtigen“" müssen ( $\$ 16$ Satz 4 HRG). Mit der Empfehlung Eine Hochschule für alle reagierte die Hochschulrektorenkonferenz (HRK) 2009 mit Maßnahmen zum Studium und stellt darin fest, dass nach Ergebnissen der Sozialerhebungen des Deutschen Studentenwerks „die besonderen Belange von Studierenden mit Behinderung/ chronischer Krankheit [...] in den Hochschulen häufig nicht ausreichend berücksichtigt [werden]“ (Hochschulrektorenkonferenz, 2009, S. 2).

Für die Qualitätssicherung und Akkreditierung der Studiengänge in den modularisierten Bachelor- und Mastersystemen muss seit 2009 ebenfalls der Nachweis von Barrierefreiheit und Berücksichtigung von Diversität in der Beratung, in den hochschuleigenen Auswahlverfahren sowie im Studium allgemein und in Prüfungssituationen im Besonderen erfolgen (vgl. Akkreditierungsrat, 2013). Gleichzeitig stellen die neuen Studiengänge mit ihren teils wenig flexiblen Prüfungs- und Leistungsverpflichtungen ein höheres Risiko für die Teilhabegerechtigkeit dar: So werden einerseits höhere Anforderungen an das Diversity Management gestellt, die die Teilhabegerechtigkeit erhöhen (sollen), gleichzeitig sind aber durch das neue Studiensystem z. B. mehr Studierende auf Nachteilsausgleiche o. Ä. angewiesen, wo vorher durch individuelle Verschiebung oder direkte Absprachen manches kompensiert werden konnte.

Die Bonner Erklärung zur inklusiven Bildung in Deutschland fordert ebenfalls den Zugang zu qualitativ hochwertiger Bildung „unabhängig von Geschlecht, Herkunft, sozialen und ökonomischen Voraussetzungen, Behinde- 
rung oder besonderen Lernbedürfnissen“ (Unesco-Kommission, 2014, S. 1). Hier wird das Hochschulwesen explizit als Bereich genannt, in dem „strukturelle und inhaltliche Anpassungen" (Unesco-Kommission, 2014, S. 1) erforderlich seien. Der Bonner Erklärung zufolge liegen innerhalb Deutschlands die Entwicklungen in den einzelnen Bundesländern weit auseinander, im Vergleich zu den europäischen Nachbarn bestehe sogar ein „erheblicher Nachholbedarf" (Unesco-Kommission, 2014, S. 1). Auch im Musikhochschulsektor sind die Entwicklungsstände recht heterogen, ein institutionalisierter Wissenstransfer findet noch kaum statt, obwohl einzelne Hochschulen das Thema nun offensiver behandeln (vgl. Musikhochschule Lübeck, 2019 oder Hochschule für Musik und Theater Rostock, 2019). Eine Ausnahme bilden die Gender Studies $^{1}$, die an einigen Hochschulen durch Professuren abgebildet sind.

\section{Instrumente und Strukturen an Musikhochschulen}

„[...] Wir handeln im gegenseitigen Respekt und mit Achtung für das Individuum und für das Miteinander aller. Wertschätzende Kommunikation und Transparenz bestimmen unser Handeln. Kritik stehen wir offen gegenüber. Gelebte Chancengleichheit und ein hohes Maß an Diversität zeichnen uns aus. Weltoffenheit spiegelt sich im internationalen Charakter unserer Hochschule. Wir stehen ein für die Freiheit von Kunst und Wissenschaft, Forschung, Lehre und Studium. Alle Formen von Diskriminierung und Machtmissbrauch lehnen wir strikt ab und treten ihnen aktiv entgegen." (Hochschule für Musik Nürnberg, 2018)

Im Zuge der Akkreditierung der Bachelor- und Masterstudiengänge sind von den Hochschulen allgemeine und lehrspezifische Leitbilder gefordert. Die Entwicklung oder Überarbeitung eines Leitbildes kann als Impuls für die breite Thematisierung von Gleichstellungs- und Diversitätsthemen genutzt werden. Gelingt es, das Leitbild in einem Bottom-up-Prozess zu entwickeln, also alle Hochschulangehörigen bei der Entstehung einzubeziehen, ermöglicht dies einen breiteren Konsens bezüglich der Inhalte und ihrer konkreten Operationalisierung. Da das Format von Leitbildern recht kompakt ist (und Papier geduldig ist), besteht die Herausforderung darin, die schön geschliffenen Formulierungen (siehe Zitat oben) im Alltag konkret mit Leben zu füllen und regelmäßig zu kommunizieren und zu evaluieren. Hierfür bieten sich die üblichen Studierbarkeitsbefragungen und Lehrevaluationsformate an, bei deren

1 Gender Studies (dt. Geschlechterforschung): eine inzwischen auch an einigen Musikhochschulen verortete interdisziplinäre Forschungsrichtung, die sich mit Fragen der Bedeutung von Geschlecht bzw. Geschlechterrolle in Kunst, Kultur, Gesellschaft und Wissenschaften befasst. 
Ausarbeitung man die Chancengleichheitsthemen konkret formulieren und abfragen sollte. Zum Beispiel kann in solchen Evaluationen nach der Zugänglichkeit von Informationen zu Gleichstellungsthemen oder der Qualität der Beratungsangebote gefragt werden. In den Lehrevaluationen können Themenwünsche aus dem Feld der Genderforschung, der inklusiven Pädagogik oder Transkulturalität abgefragt werden. Ganz allgemein wäre es interessant zu wissen, wie Studierende zum Beispiel angesprochen werden möchten, ob die Art der Kommunikation und Interaktion für sie in den Seminargruppen oder in der Verwaltung als passend empfunden wird etc.

Ein weiteres zentrales Instrument mit einem höheren Grad an Operationalisierung bieten üblicherweise die hochschuleigenen Satzungen und (Studien-) Ordnungen. Vor allem jene, die den Zugang zum Studium, zu Lehrveranstaltungen oder Projekten regeln oder die Prüfungen betreffen, sind hinsichtlich Transparenz und Teilhabegerechtigkeit kontinuierlich zu prüfen, da sie die Entscheidungsgrundlage für Gremien wie Prüfungs- und Promotionsausschüsse, Prüfungskommissionen sowie sämtliche mit dem Studium zusammenhängende Verwaltungsakte bilden. Des Weiteren sind in ihnen die sogenannten Nachteilsausgleiche zu regeln, sodass es möglich ist, Prozesse beim Zugang zum Studium oder im Studium individuell anzupassen und nicht in die alte Falle der vermeintlichen Chancengleichheit zu tappen, die Hans Traxler bereits 1975 in seiner berühmt gewordenen Tierkarikatur angeprangert hat:

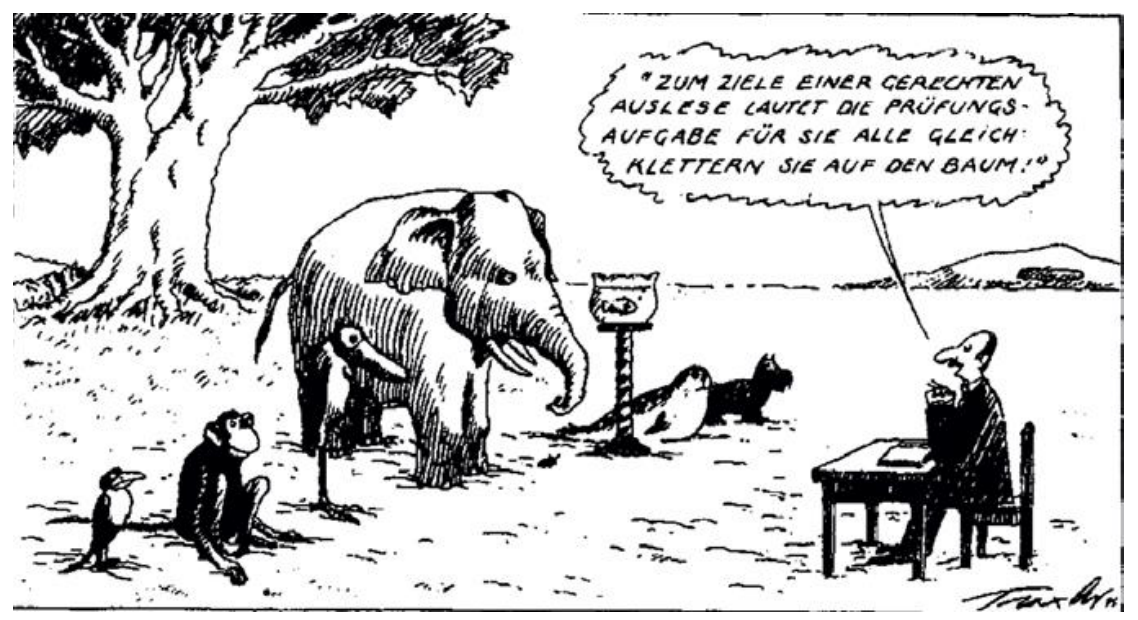

Abbildung 1: Karikatur von H. Traxler (aus Klant, 1983, S. 25) 
Aus Rainer Treptows aktueller Variante geht beispielhaft hervor, welches Umdenken diesbezüglich stattfinden muss:

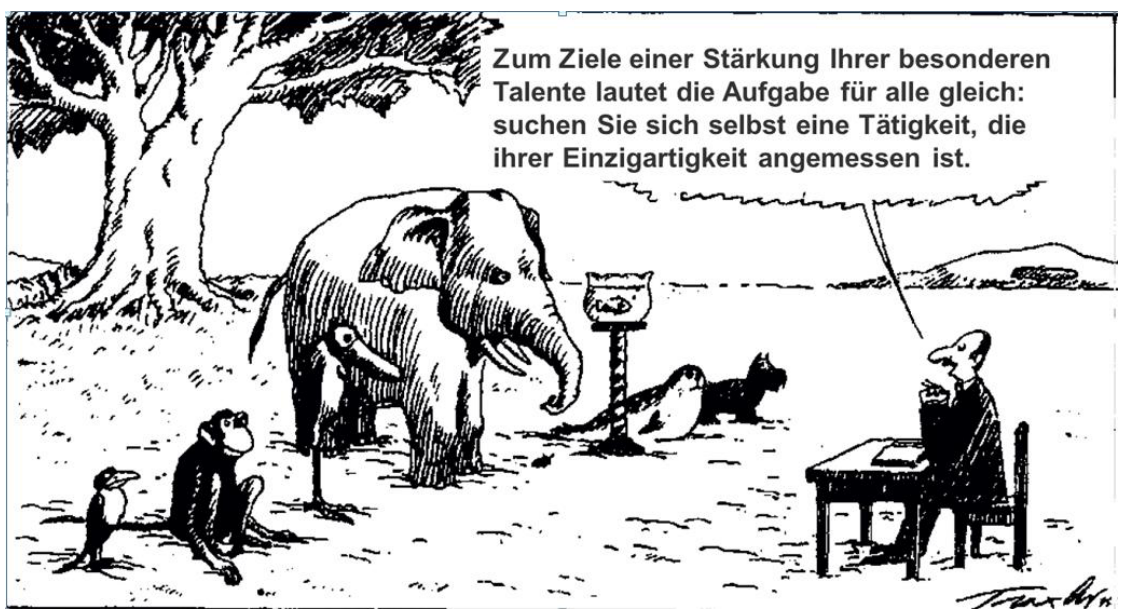

Abbildung 2: Version von Rainer Treptow (Treptow, 2015, S. 8)

Eine weitere Maßnahme der Qualitätssicherung - die den Hochschulen nicht selten Vorteile bei der Einwerbung von Drittmitteln (z. B. im sogenannten Professorinnenprogramm) eröffnet oder Zugangsvoraussetzung für Hochschulentwicklungsmaßnahmen in Form von Audits und Zertifizierungen darstellt - ist die Erarbeitung und Festschreibung von hochschulbezogenen Konzepten zum Diversitätsmanagement. Diese können etwa die Gleichstellung der Geschlechter und Familienfreundlichkeit, die (gesetzlich vorgeschriebene) Barrierefreiheit und Chancengleichheit von Studierenden mit Behinderung/chronischer Krankheit oder die Internationalisierung und den Umgang mit Geflüchteten betreffen (vgl. Hochschule für Musik Nürnberg, 2009, 2012, 2015). Solche Konzepte enthalten üblicherweise eine Bestandsaufnahme des Entwicklungsstandes (Status quo), einen Ziel- und Maßnahmenkatalog sowie entsprechende Zuständigkeiten, Überprüfungskriterien für die Zielerreichung und einen zeitlichen Horizont (z. B. von fünf Jahren), innerhalb dessen die Ziele erreicht und geprüft werden sollen. Die Texte werden in der Regel erarbeitet von einer Arbeitsgruppe oder einer bestehenden Kommission, durchlaufen anschließend die Gremien der akademischen Selbstverwaltung und können so bereits an dieser Stelle zur Bewusstseinsbildung bei möglichst vielen Hochschulangehörigen beitragen. Die Beschlussfassung obliegt meist dem Akademischen Senat. Die Herausforderung besteht anschließend darin, für eine erfolgreiche Zielerreichung die gesamte Breite der Hochschule zu mobilisieren. Dies kann 
beispielweise durch interne Fortbildungsveranstaltungen oder themenbezogene Klausurtagungen sowie die Gründung von Arbeitskreisen oder die Vergabe sogenannter Beauftragungen an Lehrende geschehen. Hierfür müssen ebenso wie für den Aufbau einer entsprechenden Infrastruktur von den Hochschulleitungen finanzielle und personelle Ressourcen (etwa Deputatsermäßigungen für Lehrende, Büros und Sachmittel, Stellen für externe Expert $\left({ }^{*}\right.$ inn)en) zur Verfügung gestellt werden.

Die Etats werden vor allem benötigt für die bestehenden bzw. neu eingerichteten Referate oder Beauftragungen, welche die sogenannten marginalisierten Gruppen vertreten. Allen voran sind hier in der Regel die Frauen- und Gleichstellungsbeauftragten zu nennen, die schwerpunktmäßig die genderspezifischen Bedarfe in Studierendenschaft, bei Lehrenden und in der Hochschulverwaltung managen. Ferner handelt es sich üblicherweise um Beauftragte für Studierende mit Behinderung, Beauftragte für Internationalisierung (sofern nicht die Zuständigkeit bei Präsidium bzw. Hochschulleitung verortet ist) oder auch Beauftragte für geflüchtete Studierende. Für diese Funktionsträgerinnen sowie für weitere Hochschulangehörige, allen voran die studienberatend Tätigen, sollte ein entsprechendes Fortbildungskonzept bestehen und ebenfalls Mittel bereitgestellt sowie ein Anreizsystem für die Teilnahme (z. B. Lehrermäßigungen, ECTS-Punkte, leistungsbezogene Gehaltsbestandteile) geschaffen werden. Die Studierenden benötigen ferner ein transparentes und bedarfsorientiertes Stipendiensystem. Hier gilt es, gegebenenfalls Stipendiengeber*innen zu akquirieren, die ein Förderinteresse an speziellen Zielgruppen, z.B. blinden oder geflüchteten Studierenden, haben sowie bei den Vergabemechanismen der Stipendien generell auf eine diversitätsadäquate Vergabe zu achten. Diesbezüglich sind die üblichen Vergabekriterien (besondere Leistungsfähigkeit bzw. besondere Bedürftigkeit) wo möglich zu überarbeiten oder entsprechend auszulegen, etwa mittels einer Quotierung hinsichtlich Geschlechtergerechtigkeit oder weiterer Merkmale.

Als Ergänzung zu den Stipendien, die meist mit finanzieller Förderung verbunden sind, können Mentoringsysteme eingerichtet werden, bei denen der persönliche Erfahrungsaustausch sowie die gemeinsame Bearbeitung von inhaltlichen Themen im Mittelpunkt stehen. Passende Mentor $\left({ }^{*}\right.$ inn)en aus dem Umfeld der Hochschule (Absolvent(*inn)en, Berufstätige in Musikberufen) unterstützen in diesem Fall aktuelle Studierende, etwa bei konkreten studienbezogenen Anforderungen oder aber in der Übergangsphase in den Beruf. Die Suche nach geeigneten - weil evtl. in ähnlicher Weise wie ihre Mentees herausgeforderten - Mentor( ${ }^{*}$ inn)en gelingt leichter, wenn sich die Hochschule generell mit ihrem Umfeld gut vernetzt und Kooperationsprojekte sowie die Einbindung externer Kompetenz in den jeweils spezifischen Fragen der Hochschulentwicklung selbstverständlich betreibt. Die hochschuleigenen Qualitäts- 
sicherungsmaßnahmen sehen in der Regel sowieso die Einbindung von externer Expertise bei der Akkreditierung und Überarbeitung der Studiengänge vor, sodass hier Synergien entsprechend genutzt werden können.

Diese Ausführungen zeigen, dass Chancengerechtigkeit und Diversität Querschnittsthemen der Hochschule darstellen, die sowohl in den bestehenden Strukturen der akademischen Selbstverwaltung, der allgemeinen Hochschulverwaltung als auch auf der Ebene der Forschung und Lehre ihren Niederschlag finden müssen. Insofern gilt es, in möglichst vielen Studienrichtungen, insbesondere aber in allen pädagogischen Modulen oder Studiengängen, sowohl ein spezielles Lehrveranstaltungs- und Praktikumsangebot zu implementieren als auch bei den hier tätigen Lehrenden das Bewusstsein dafür zu schärfen, dass Diversität als Querschnittsthema in allen Lehrveranstaltungen zu berücksichtigen ist. Dies fängt bei (gender-)sensibler Sprache an und kann über die Durchführung von Projekten mit speziellen Zielgruppen, etwa im Rahmen der Fachdidaktiken, bis hin zum interdisziplinären Lehr-/Lernexport mit spezialisierten Studiengängen anderer Hochschulen (z. B. Soziale Arbeit, Frühkindliche Bildung und Erziehung, Musiktherapie, Community Music) gehen. Im Zuge der Einrichtung von pädagogischen Masterstudiengängen sind weitere Schwerpunktsetzungen denkbar, wie etwa im Masterstudiengang Musizieren mit heterogenen Gruppen an der Hochschule für Musik Nürnberg. In die eher forschungsorientierten musikpädagogischen Masterstudiengänge können Projekte zur Implementierungs- und Evaluationsforschung integriert werden oder Praxisprojekte beforscht werden.

Eine generelle Herausforderung bleibt es, die wechselnden Leitungen und Präsidien der Musikhochschulen dafür zu sensibilisieren, dass hier eine strategische Entwicklungsaufgabe vorliegt, die nur durch Konsequenz und eine Vielzahl an Ansatzpunkten gemeistert werden kann. Nur so kann garantiert werden, dass die Weiterentwicklung des Diversitätsmanagements an Hochschulen nicht von einzelnen engagierten Personen abhängt, sondern auf breiter Basis wirksam werden kann. Problematisch und hinderlich sind die beständig knappen finanziellen und personellen Ressourcen, in den meisten Fällen besteht ohne Drittmittelakquise kaum Handlungsspielraum. Ein weiteres Spezifikum des Feldes ist, dass die sehr individuellen Bedürfnisse der Hochschulangehörigen häufig nicht sichtbar sind. Während etwa durch Blindheit, Gehörlosigkeit oder Besonderheiten im Bewegungsverhalten auch für Außenstehende schnell Hinweise auf möglichen Anpassungsbedarf gegeben werden, bleiben viele Bedürfnisse, die z. B. aufgrund psychischer Dispositionen (Ängste, Zwangserkrankungen, Depression) oder chronischer Erkrankungen entstehen, häufig unsichtbar. Auch hinsichtlich anderer Unterscheidungsmerkmale sind Teilhabekriterien häufig nicht klar erkennbar oder werden ungern kom- 
muniziert. Noch immer verzichten viele auf die Inanspruchnahme von Unterstützung oder Individualisierung aus Angst vor (Wettbewerbs-)Nachteilen oder Stigmatisierung. Hier besteht weiterhin die Notwendigkeit konsequenter Aufklärung und Enttabuisierung.

\section{Möglichkeiten}

Begreift man die unterschiedlichen Bedürfnisse der Musikhochschulangehörigen als Chance und Kreativitätspotenzial sowie Diversität als intelligentes System, durch das Fehler schneller erkennbar und ausgeglichen werden als in eher homogenen Zusammensetzungen, so besteht die Chance, dass aus vermeintlichen Einschränkungen oder Besonderheiten neue alternative Lösungswege und Impulse entstehen können. Insbesondere im Bereich der Hochschuldidaktik ergibt sich eine Fülle an Ansatzpunkten für die Lehre, die schließlich allen Studierenden zugutekommen können. Im Zuge der Digitalisierung von Lehre und Hochschule besteht ohnehin die Herausforderung, neue Formate $\mathrm{zu}$ erproben, diese zu bewerten und tradierte Studienformate zu flexibilisieren. Dies bietet ein enormes Entwicklungspotenzial hin zu mehr Chancen- und Teilhabegerechtigkeit: So ist es im Rahmen von blended learning leichter möglich, unabhängig von Präsenz am Hochschulort zu studieren, den Studienverlauf zeitlich zu flexibilisieren oder Materialen noch barrierefreier und anpassbarer aufzubereiten.

Häufig vernachlässigt und im Zuge von Diversitätsfragen oft unterschätzt ist das Thema Musiker( ${ }^{*}$ innen) gesundheit, das sich in der Vorbereitung auf den Beruf inzwischen als essenziell erwiesen hat und daher als Schlüsselqualifikation in allen musikbezogenen Studiengängen verankert ist. Hier bietet sich ebenfalls die Chance, Synergien und Schnittmengen zu identifizieren und zu kommunizieren.

Insgesamt hängt die Profilierung einer Musikhochschule bezüglich Chancengleichheit und Teilhabe von der offensiven Kommunikation der Thematik nach außen ab, etwa indem auf der (barrierefreien) Webseite das Thema prominent platziert wird (vgl. hierzu etwa die Webauftritte der Musikhochschulen Lübeck und Rostock). So können geeignete Bewerber*innen und Lehrpersonal rekrutiert werden, die eine qualitätsgesicherte und teilhabegerechte Hochschule gemeinsam voranbringen.

Ziel bleibt es, an Musikhochschulen ein Lern- und Arbeitsumfeld zu generieren und zu sichern, das es Studierenden, Lehrenden und Mitarbeitenden in der Verwaltung unabhängig von Geschlecht, Alter, Herkunft, Hautfarbe, Religion, Behinderung/chronischer Krankheit, individuellen Begabungen und se- 
xueller Identität ermöglicht, Zugang zu finden, zusammen zu arbeiten und das jeweilige Potenzial vollumfänglich zu entfalten.

\section{Literatur}

Akkreditierungsrat (2013). Regeln für die Akkreditierung von Studiengängen und für die Systemakkreditierung. Verfügbar unter: http://www.akkreditierungsrat.de/file admin/Seiteninhalte/AR/Beschluesse/AR_Regeln_Studiengaenge_aktuell.pdf [14.05.2020].

Bundesrepublik Deutschland. Allgemeines Gleichbehandlungsgesetz. Verfügbar unter: https://www.gesetze-im-internet.de/agg/ [14.05.2020].

Bundesrepublik Deutschland. Grundgesetz für die Bundesrepublik Deutschland. Verfügbar unter: https://www.gesetze-im-internet.de/gg/BJNR000010949.html [14.05.2020].

Bundesrepublik Deutschland. Hochschulrahmengesetz. Verfügbar unter: https:// www.gesetze-im-internet.de/hrg/ [14.05.2020].

Hochschule für Musik Nürnberg (2009). Gleichstellungskonzept der Hochschule für Musik Nürnberg für die Jahre 2009-2014. Verfügbar unter: http://www.hfm-nu ernberg.de/fileadmin/user_upload/Text-Pool/Download_Formulare/Gleichstel lungskonzept_HfM_Nuernberg.pdf [14.05.2020].

Hochschule für Musik Nürnberg (2012). Konzept der Hochschule für Musik Nürnberg zur Sicherung der chancengleichen Teilhabe von Studierenden mit Behinderung/chronischer Krankheit an der Hochschulbildung. Verfügbar unter: http:// www.hfm-nuernberg.de/fileadmin/user_upload/Text-Pool/Download_Formu lare/Konzept_zur_Sicherung_der_chancengleichen_Teilhabe_von_Studieren den_mit_Behinderung_chronischer_Krankheit_an_der_Hochschulbildung.pdf [14.05.2020].

Hochschule für Musik Nürnberg (2015). Konzept zur Internationalisierung der Hochschule für Musik Nürnberg 2015-2025. Verfügbar unter: http://www.hfmnuernberg.de/fileadmin/user_upload/Text-Pool/Sonstiges/Konzept_zur_Inter nationalisierung_nach_Senat.pdf [14.05.2020].

Hochschule für Musik Nürnberg (2018). Leitbild. Verfügbar unter: http://www.hfmnuernberg.de/fileadmin/user_upload/Text-Pool/Sonstiges/Leitbild_HfM_Nu ernberg.pdf [14.05.2020].

Hochschule für Musik und Theater Rostock (2019). hmt inklusiv. Verfügbar unter: https://www.hmt-rostock.de/hochschule/hmt-inklusiv/ [14.05.2020].

Hochschulrektorenkonferenz (2009). Eine Hochschule für Alle. Empfehlung der 6. Mitgliederversammlung am 21.4.2009 zum Studium mit Behinderung/chronischer Krankheit. Verfügbar unter: https://www.hrk.de/uploads/tx_szconvention/ Entschliessung_HS_Alle.pdf [14.05.2020].

Klant, M. (1983). Schul-Spott. Karikaturen aus 2500 Jahren Pädagogik. Hannover: Fackelträger. 
Musikhochschule Lübeck (2019). Inklusion als Querschnittsthema. Verfügbar unter: https://www.mh-luebeck.de/de/studium/inklusion/inklusion-als-querschnitts thema/ [14.05.2020].

Treptow, R. (2015). Chancengleichheit und kulturelle Bildung. Vortrag. Verfügbar unter: http://kulturteil-nms.de/cms/files/vortrag_chancengleichheit_und_kultu relle_bildung_prof._treptow.pdf [14.05.2020].

Unesco-Kommission (2014). Bonner Erklärung zur inklusiven Bildung in Deutschland. Verfügbar unter: https://www.hamburg.de/contentblob/4301000/29e84c 3f9da3f0729fec347a5ad7ce2b/data/unesco-erklaerung.pdf [14.05.2020].

Vereinte Nationen (2006). Behindertenrechtskonvention. Übereinkommen über die Rechte von Menschen mit Behinderungen. Verfügbar unter: https://www.behin dertenrechtskonvention.info/vertragstexte/ [14.05.2020]. 
Podiumsdiskussion

\section{Die Qualität des Nicht-Synchron-Seins - Gedanken zur Funktionalität und Widerspenstigkeit}

In einer Podiumsdiskussion wurden einige der Autorinnen und Autoren dieses Tagungsbandes sowie weitere ausgewählte Personen (Günther Klausner, Leiter der städtischen Musikschule in Hall in Tirol (Ö), Robert Wagner, Leiter der Musikschule Fürth (D) sowie Dieter Bucher, Lehrer für Elementare Musikpädagogik am Landeskonservatorium Klagenfurt) von Heike Henning zu in den Vorträgen angeklungenen Aspekten und Phänomenen befragt. In dieser verschriftlichten Podiumsdiskussion geht es neben dem weiten Thema Inklusion im Besonderen um die Bedeutung des Synchron-Seins in der Kunst, sowie um Ausschlüsse, welche durch solche Paradigmen entstehen.

Heike Henning: Laut Michael Turinsky ergibt sich Qualität daraus, nicht synchron zu sein. Starten wir mit einer Blitzlichtrunde: Woraus ergibt sich Ihrer Meinung nach die Qualität künstlerischer Praxis? Was sind Qualitätsmerkmale künstlerischer (Musizier-)Prozesse?

Shirley Salmon: Für mich sind Kontakt, Begegnung und Berührtwerden wichtige Elemente.

Renate Reitinger: Der Aufbau und das Abebben von Spannung sind für mich wesentliche Elemente. Es braucht Variation und Unterschiedlichkeit. Deswegen ist es ganz klar, dass Kunst nur durch die Abkehr vom Synchronen funktionieren kann.

Julia Lutz: Wesentlich ist, sich einzubringen, sich selbst zu erleben, sich selbst in und mit der Gruppe bzw. die Gruppe selbst zu erleben. Das kann synchron genauso wie nicht synchron erfolgen.

Robert Wagner: Ohne die Qualität des Synchronen wäre es nicht möglich, nicht synchron zu sein. Also braucht das eine das andere.

Günther Klausner: Mir fällt da ganz spontan ein Wort ein, nämlich Zulassen. Ob synchron oder nicht synchron ist dann eigentlich nicht mehr relevant.

Dieter Bucher: In vielen Gruppen, in denen ich arbeite, ist Synchronität gar nicht möglich, da geht es um Ausdruck einer Stimmung oder Emotionalität. Und wenn möglich um ein aufeinander Hören und darum, in Dialog treten zu können. 
Michael Turinsky: Für mich kann Qualität unter anderem durchaus daraus bestehen, sich immer wieder aufeinander einzustimmen, sich immer wieder aufeinander einzutunen - aber eben auf eine nicht strikt marschartige, glatte Art und Weise, sondern auf eine Art und Weise mit kleinen Verschiebungen, kleinen Differenzen und Disynchronisierungen, die Synchronisierung erlaubt.

Heike Henning: Die zwanghafte Funktionstüchtigkeit unserer Gesellschaft begegnet uns an vielerlei Orten und auf unterschiedliche Arten. Welche Funktion erfüllt sie? Welche Paradigmen sind hierfür leitend? Wie wesentlich sind diese für künstlerisch-pädagogische Prozesse?

Renate Reitinger: Es wird billiger, wenn alle funktionieren. Leider spielen diese finanziellen Vorgaben eine Rolle. So wird eine Effizienz vorgetäuscht, die leider andere Dinge verhindert.

Robert Wagner: Funktionstüchtigkeit ist mir jetzt etwas zu negativ dargestellt. Ein Mensch, der beatmet werden muss, ist ganz dankbar, dass die Maschine funktioniert.

Günther Klausner: Ohne Funktionstüchtigkeit würden ganz viele Abläufe ja gar nicht funktionieren. Zum Beispiel haben wir an unseren Musikschulen den großen Vorteil, Methodenfreiheit zu haben. Wir müssen schon funktionieren und haben Zielvorgaben, aber in einem sehr weiten Rahmen. Wen oder was wir unterrichten ist für mich keine theoretische Frage. Inklusion ist für mich tägliche Praxis! An Musikschulen arbeiten wir automatisch sehr inklusiv. Ich habe den österreichischen Ligabericht 2018 im Kopf - ca. 20 Prozent der Jugendlichen in Österreich haben psychische Probleme. Wenn ich das bedenke, praktiziere ich eigentlich täglich Inklusion und das muss funktionieren. Ohne Funktionstüchtigkeit wäre es nicht möglich.

Dieter Bucher: Mit dem Zwang, funktionieren zu müssen, können wir alle mehr oder weniger gut umgehen. Oft können gerade hochintelligente oder -gebildete Menschen - häufig sind das Frauen, die vor der Matura stehen oder studieren - dem Druck nicht standhalten. Das sind die Leute, die wir dann in der Jugendpsychiatrie antreffen. Sie sind aus der Bahn geworfen worden, weil sie das Gefühl haben, nicht ausreichend funktionieren zu können. Physische Folgen sind oft bspw. Magersucht oder Bulimie. Jugendliche Menschen, die es aufgrund ihrer Bildung, ihrer intellektuellen Ausstattung oder aufgrund ihres spezifischen Umfeldes nicht schaffen, diese verlangte Funktionstüchtigkeit zu erfüllen, werden mitunter straffällig. Um dem Druck zu entfliehen, nehmen sie Drogen und werden teilweise sehr unangenehm. Und einige Menschen mit Behinderungen haben gar nicht die Chance, funktionstüchtig zu sein. Weil uns aber heute bswp. durch Facebook und Instagram vorgegeben wird, man müsse 
sich inszenieren und schön, sexy sowie klug sein und alles können - am besten alles zugleich - zerbrechen sehr viele an dieser inszenierten Funktionstüchtigkeit. Viele können diese Art des Funktionierens nie erreichen. Manche hatten sie einst erreicht und dann wieder verloren, weil sie jetzt alt oder in einer Demenzsituation sind oder was auch immer. Mich gruselt vor dem Wort Funktionstüchtigkeit.

Shirley Salmon: Funktionstüchtigkeit ist abhängig davon, in welchem System wir uns befinden. Ich denke, wenn wir über Funktionieren diskutieren, ist es immer auch sehr wichtig, dass man das einzelne Individuum nicht vergisst, denn auch in Gruppen muss man den einzelnen Menschen sehen - und da liegt die Gefahr, wenn es um Funktionalität oder Funktionstüchtigkeit geht.

Michael Turinsky: Und darin liegt der Unterschied zwischen dem, was ich von einer Maschine verlange und dem, was ich von einem Menschen möchte: Die Maschine als Beatmungsgerät hat keine Individualität.

Robert Wagner: Der Mensch ist keine Maschine und sollte auch gar nicht damit verglichen werden. Ich wollte nur ein bisschen darauf hinweisen, dass wir alle davon profitieren, dass irgendwelche Kolleginnen und Kollegen um uns herum funktionieren. Wir alle profitieren davon, dass die Vorbereitung auf diese Tagung so gut war und dass das Zeitmanagement funktioniert. Das Gesamte ist im Blick zu behalten und muss funktionieren. Funktionalität gehört meines Erachtens nach zum Leben. Nur der Leistungswahn, der in unserer Gesellschaft existiert, ist zu kritisieren und macht tatsächlich krank - nicht die bloße Funktionstüchtigkeit und die Notwendigkeit, in der Früh aufzustehen und teilzugeben.

Julia Lutz: Was heißt eigentlich funktionieren? Gibt es einen Normbereich, in dem zum Beispiel eine musizierende Gruppe funktioniert? Es ist doch denkbar, dass ich ein Instrument spiele, das nicht mehr funktioniert und dadurch eine neue Spielweise entwickle. Da ist immer die Frage: Was ist mit Funktionieren gemeint? Und: Kann ich die Bedeutung von Funktionieren ausdehnen und erweitern?

Heike Henning: Wie lässt sich dieser Normbereich beim Musizieren definieren? Besteht ein Widerspruch zwischen Leistung und Inklusion? Sie, Herr Turinsky, nehmen in dieser Runde eine Sonderrolle ein - diese ergibt sich nicht dadurch, dass Sie im Rollstuhl sitzen, sondern dass Sie ein Tänzer sind. Wir anderen sind Musikpädagog( ${ }^{*}$ inn)en. Sie schreiben, dass Ihr Schwerpunkt darin liegt, $z u$ untersuchen, worin das spezifische In-der-Welt-Sein von Menschen mit Behinderung liegt. Können Sie beschreiben, was dieses spezifische In-der-Welt-Sein ist? 
Michael Turinsky: Ich gehe davon aus - Stichwort Individualität -, dass sich das mit unserem Körper In-der-Welt-Sein für uns alle ganz unterschiedlich anfühlt. Auf der einen Seite stimmen wir alle darin überein, dass unsere Gesellschaft Barrieren aufrichtet und dass diese manche Menschen behindern. Unterschiedliche Behinderungsformen produzieren ganz unterschiedliche Effekte und fühlen sich auch deshalb unterschiedlich an. Das auf eine Weise zusammenzudenken heißt für mich, Behinderung als eine jeweils spezifische Art von Widerständigkeit zu sehen. Für eine blinde Person fühlt es sich mehr an wie ein Widersetzen gegen gesellschaftliche Normen der Sichtbarkeit und des Sehen-Könnens - für mich hat es wiederum mit Geschmeidigkeitsnormen oder Normen von Bewegungsfluss zu tun. Für uns alle fühlt es sich anders an, mit unserem Körper in unserer Welt zu sein.

Heike Henning: Gibt es etwas, bei dem Sie sagen, dass es Menschen mit Behinderung eint? Von dem bisher Gesagten könnte es das Erleben von Barrieren sein. Gibt es noch mehr?

Michael Turinsky: Also ich glaube, es ist schwierig, ein sozusagen essentialistisches Element aus diesem Gemeinsamen zu konstruieren und davon auszugehen, dass tief in unseren Wesen drinnen etwas Gemeinsames steckt. Daran habe ich Zweifel. Aber natürlich machen wir durch den Umstand, dass wir in der gleichen Gesellschaft leben, auch gleiche Erfahrungen. Und eine Weise zu denken ist eben, dass wir zum Beispiel - sofern wir zufälligerweise heterosexuell sind - eben keine Erfahrung mit der heterosexuellen Norm machen, sondern als behinderte Menschen uns vor allem an der Norm der körperlichen oder geistigen Funktionstüchtigkeit reiben. Diese Norm ist eigentlich eben genau der Widerstand. Sie ist nicht unbedingt voluntaristisch gedacht, sondern als materialistische Eigenschaft. Diese Widerständigkeit ist in einem QueerKontext vielleicht der Widerstand gegen die Norm der Zweigeschlechtlichkeit. Uns Menschen mit Behinderung eint eben die Widerständigkeit gegen die Funktionstüchtigkeit.

Heike Henning: Ich habe eine Doppelidentität als Musikerin und als Wissenschaftlerin. Mich würde abschließend interessieren, wo Sie auf mehr Barrieren treffen, in der Kunst oder in der Wissenschaft?

Michael Turinsky: Eindeutig in der Wissenschaft.

Heike Henning: Sie, Herr Turinsky, mögen den Begriff der Inklusion nicht, trotzdem muss ich ihn noch einmal verwenden. Inwieweit ist die Präsenz von Menschen mit Behinderung im Bereich der Lehrkörper wesentlich für das Gelingen inklusiver Prozesse? 
Michael Turinsky: Sicherlich ist das ein wichtiger Punkt, wobei man natürlich anerkennen muss, dass wir uns erst mal selbst das Know-how des Lehrens aneignen müssen. Nicht jeder kann lehren! Auch ich finde es herausfordernd zu unterrichten, obwohl ich es sehr gerne tue.

Heike Henning: Herr Wagner, sie haben gesagt, dass der inklusive Begriff keine korrekte Anwendung im Sprachgebrauch hat, solange sich der Begriff ausschließlich auf Menschen mit Behinderung bezieht.

Robert Wagner: Ich glaube solange wir nicht verstehen, dass mit dem Begriff Inklusion jede $\left.{ }^{*} \mathrm{r}\right)$ von uns gemeint ist, werden immer neue Schubladen geöffnet statt welche geschlossen. Deswegen möchte ich vom Teil-Sein sprechen: Jede $\left.{ }^{*} r\right)$ ist Teil aller Menschen, weil sie/er da ist. Niemand muss inkludiert werden, sondern wir müssen lernen, diesen Begriff Inklusion als Leitbild der Gesellschaft zu sehen - nicht starr, sondern immer in Bewegung. Und insofern ist eine inklusive Musikschule eine bewegte Schule. Und es gibt inklusive Prozesse, inklusives Handeln und Denken, das eben diese Entwicklung vorantreibt. Daher brauchen wir letztendlich diesen Inklusionsbegriff (noch). Und auch wenn ich dem etwas abgewinnen kann, dass Herr Turinsky gesagt hat, er möchte den Begriff gar nicht mehr verwenden, vergeben wir uns meiner Meinung nach eine Chance, wenn wir das nicht tun, weil wir zurzeit immer noch diese UN-Ratifizierung haben. Jetzt ist es keine Frage mehr, ob wir inklusiv sein wollen, sondern nur noch, wie Inklusion gestaltet wird. Jetzt gibt es ein Rechtssystem, das Menschen mit Behinderungen garantiert, bestimmte Dinge zu bekommen. Jetzt ist klar, das ist ein Menschenrecht. Deswegen würde ich mit Vehemenz darum bitten, den Begriff Inklusion zu behalten und ihn richtig verstehen zu lernen.

Heike Henning: Der Artikel 30 Absatz 2 der UN-BRK beschreibt die staatliche Pflicht, geeignete Maßnahmen zu treffen, Menschen mit Behinderungen die Möglichkeit zu geben, ihr künstlerisches und kreatives Potenzial zu entfalten. Wie können musikbezogene Maßnahmen aussehen und welchen Beitrag leisten Musikschulen zur inklusiven Gesellschaft?

Robert Wagner: Die gesamte Gesellschaft hat eine Mitverantwortung für das Gelingen einer inklusiven Gesellschaft. Wir haben gemeinsam die Aufgabe, tätig zu werden. Wir Musikschulleitende und -lehrkräfte tragen für den Bereich der Musikschulen diese Verantwortung und müssen uns fragen: Bauen wir Barrieren auf oder ab? Machen wir annehmbare Angebote? Es beginnt mit der Werbung. Wenn man in die Zeitung schreibt, die Musikschule Fürth öffnet sich für Menschen mit Behinderung, kann man davon ausgehen, dass das niemand liest, dass zumindest die Menschen, für die das gedacht ist, das nicht lesen. Wir müssen aktiv auf Menschen mit Behinderungen zugehen, sie gezielt 
aufsuchen. Wir müssen in Förderschulen und Altenheime gehen. Wir setzen uns dabei dem Vorwurf aus, dass wir zu diesen randständigen Zielgruppen gehen, anstatt diese Menschen in die Musikschulen reinzuholen. Aber das ist ein vorübergehender erster Schritt. Wir müssen die Menschen zunächst abholen, weil sie nicht von allein kommen würden. Das Ziel ist natürlich, dass die Leute, die wir dort finden, reguläre Musikschüler*innen werden.

Heike Henning: Herr Klausner, gab es an der Landesmusikschule Kitzbühel entsprechende Maßnahmen? Welche Interventionen sind erforderlich, damit eine Musikschule noch breiter mit der Gesellschaft in Kontakt treten kann?

Günther Klausner: Ich könnte das von der systemischen Seite beantworten, z. B. die verantwortliche Politik muss den Musikschulen A und B zur Verfügung stellen. Das wäre aber erstens zu kurz gegriffen und zweitens dennoch nicht machbar für einzelne Lehrkräfte. Ansetzen müssen wir an den eigenen Gedankengängen, müssen selbst aktiv werden, bereit sein, uns zu öffnen, weg vom Instruktor $\left({ }^{*}\right.$ inn $)$ endenken. Wenn wir zuerst dieses ganzheitliche Denken schaffen, ist der nächste Schritt in Richtung Öffnen-für-alle-Schichten-derGesellschaft ein leichterer.

Heike Henning: Herr Bucher, Sie arbeiten u.a. in Justizanstalten etc. Basis ihrer Arbeit ist vermutlich eine sehr unvoreingenommene Haltung. Gibt es weitere Geheimnisse, die bei der Arbeit mit heterogenen Gruppen helfen?

Dieter Bucher: Eigentlich keine Geheimnisse. Liebe zu den Menschen, zur Musik und Unvoreingenommenheit. Ich brauche keine sonderpädagogische Ausbildung, um in heterogenen Gruppen auch mit Menschen mit geistiger Behinderung arbeiten zu können. Es sind Menschen und die sind in erster Linie jung oder alt. Das Lebensalter spielt eine große Rolle, weit dahinter folgt irgendwann der Aspekt der Behinderung.

Heike Henning: Sie haben auch federführend den Lehrplan für Elementare Musikpädagogik mitgestaltet. Inwieweit spiegelt sich dort der Beschluss der UN-Behindertenkonvention? Gibt es diesbezüglich nennenswerte Änderungen?

Dieter Bucher: Also wir haben den aktuellen, zehnjährigen Lehrplan überarbeitet und modernisiert. Wir haben in unserer praxisbezogenen Verblendung diesen menschenrechtsbezogenen Paragraphen nicht im Fokus gehabt. Der Lehrplan gilt für Kinder und Erwachsene - unabhängig vom Alter. Damit schließen wir alle ein, ohne darauf explizit hinzuweisen, dass wir Inklusion damit meinen. Im fachspezifischen Teil Elementares Musizieren mit Menschen mit Behinderung haben wir Verschiedenes erwähnt: Inklusion, Normalitätsgebot, Respekt der Lehrpersonen im zwischenmenschlichen Umgang (keine Hilfestellungen aufdrängen etc.). Ich meinte vorhin praxisbezogene Verblendung, weil 
in der Praxis finden wir kaum stark heterogene Gruppen (bspw. mit kognitiven Behinderungen, Migrationshintergrund usw.). Wobei ich mir nicht sicher bin, ob das mit Inklusion gemeint ist. Inklusion bedeutet ja allgemeine kulturelle Teilhabe für alle - es müssen ja nicht immer heterogene Gruppen sein, obwohl ich mir das sehr wünschen würde. Was aber jetzt natürlich durch dieses Symposium angestoßen wurde, dass wir schon ganz weit vorne den Begriff Inklusion für eine Bewusstseinsbildung verankern müssen.

Heike Henning: Frau Reitinger, Inklusion ist für Sie eine innere Haltung. Wie schafft man es, diese innere Haltung bei künftigen Musikschullehrenden aufzubauen?

Renate Reitinger: Das ist eine große Herausforderung, vor allem wenn man sich vorstellt, wer alles an eine Hochschule kommt. Da alle durchs Nadelöhr der Eignungsprüfung müssen, könnte man doch davon ausgehen, die können und wollen alle das Gleiche - da ist schon der erste Wandelpunkt, der bei uns Lehrenden einsetzen muss. Dann sind da noch die Studierenden mit ihren ganz eigenen Zukunftsplänen. Da besteht zuerst mal die Herausforderung, dieses Offensein für diese Haltung näherzubringen. Wir bieten verschiedene Ansatzpunkte: Wir versuchen, den frühen Kontakt mit der Praxis in Institutionen in unseren Musikschulkooperationen anzustoßen. Wir sind bemüht, im ersten und zweiten Semester schon die Erfahrung zu ermöglichen, sich selbst als anders wahrzunehmen, bspw. in Lehrveranstaltungen, in denen sehr viele unterschiedliche Studierende sind (aus den Bereichen Jazz, Klassik und offizielle Studierende mit Behinderung). Die Erfahrung zu machen, miteinander $\mathrm{zu}$ musizieren und zu schauen, wie sich das anfühlt. Und das Anleiten von heterogenen Gruppen, um zu betonen, dass jede Gruppe heterogen ist. Das widerspricht theoretisch dem, was Herr Bucher gerade sagte: Einfach sehen, wer ist da, wer will was, wer kann was? Und dann mit dem umzugehen, was da ist. Aus der Elementaren Musikpädagogik stammt unser Methodenkoffer. In dieser Disziplin findet sich auch eine große Bereitschaft, diese Sichtweise auf alle Menschen zu etablieren.

Heike Henning: Sie haben im Rahmen der Funktion als Vorsitzende für die Kommission für Gleichberechtigung ein Konzept für chancengleiche Teilhabe an der Musikhochschule von Studierenden mit Behinderung und chronischer Krankheit erarbeitet. Was beinhaltet und was bewirkt das Konzept?

Renate Reitinger: Ich muss zugeben, dass wir uns den Titel nicht selber ausgesucht haben. Von der Rektorenkonferenz der deutschen Musikhochschulen gab es eine Rahmenvorgabe, die hieß Eine Hochschule für alle. Da waren wir also aufgefordert, als Mitgliedshochschulen in einen solchen Hochschulentwicklungsprozess einzusteigen und ein Konzept zu entwickeln. Das ha- 
ben wir als Impuls aufgenommen, da bis dahin dieses Thema nicht in unserem Bewusstsein war, weil auch bei uns eine Orientierung an den Mehrheiten praktischer und bequemer ist, als nach den Einzelfällen zu schauen. Die Kommission hat sich darauf verständigt, Leitbildformulierungen zu schreiben, die eine Vision für die Zukunft sind. Der zweite Teil war sehr konkret und verwaltungsorientiert gedacht: über das Wort Nachteilsausgleich nachzudenken und es zu definieren, mit dem Ziel, eine Haltung in den Eignungsverfahren, Prüfungskommissionen etc. zu erreichen, bei der es eben normal ist, dass es Sonderfälle geben kann, dass besondere Bedingungen bei Prüfungen erforderlich sind. Das Konzept ist von 2012 und die Erfahrungen zeigen, dass sich schon etwas verändert hat, dass es mittlerweile für die Prüfungsausschüsse selbstverständlich ist, dass es solche Sonderregelungen geben kann, dass das nichts mit Bevorteilung zu tun hat. Die größere Herausforderung ist, dass die Studierenden das in Anspruch nehmen. Aber auch das entwickelt sich, so dass einzelne kommen und sagen, sie mögen diese Prüfung in einer anderen Form machen. Wir haben wenige Fälle von Behinderung: Es gibt Menschen mit Sehbehinderung; das ist vielleicht der typischste Fall. Und wir haben einen hohen Anteil an psychischen Erkrankungen, die eigentlich den Hauptteil ausmachen. Das sind natürlich Themen, die nicht so gerne offensiv angesprochen werden. Zum Glück haben wir gemeinsam mit der Verwaltung erreicht, dass es für sie auch schon einfacher wird, aber der Weg ist noch lang.

Heike Henning: Frau Lutz, Sie bieten eine Lehrveranstaltung mit der Bezeichnung Basisqualifikation Musik und Inklusion an. Wie lassen sich diese Basisqualifikationen beschreiben?

Julia Lutz: Ich würde eigentlich gar nicht vom Begriff Basisqualifikation oder damit verbundenen Kompetenzen sprechen wollen. Es geht vielmehr um die eigene innere Haltung, eine Haltung der Offenheit dem gegenüber, was zum Beispiel Kinder in einer Grundschulklasse an völlig verschiedenen Voraussetzungen mitbringen. Also Bewusstsein schaffen für diese vielfältigen Voraussetzungen, die in einer solchen Klasse vorhanden sind. Uns ist sehr wichtig, dass die Studierenden in diesen Grundkursen möglichst viele Erfahrungen sammeln können. Diese Studierendengruppen sind selbst sehr heterogen und bringen gerade in Bezug auf Musik sehr unterschiedliche Voraussetzungen mit (kaum oder viel Musiziererfahrungen). So sammeln sie in dieser heterogenen Gruppe Erfahrungen beim Musizieren, welche gemeinsam reflektiert werden. Diese Reflexion ist uns wichtig. Mit dem darin gewonnenen Blick wird dann überlegt, wie man in heterogenen Grundschulklassen musikalische Erfahrungen schaffen und reflektieren kann. Ein wesentlicher Punkt ist es, die Vielfalt in der Gruppe der Kinder wahrzunehmen und damit umgehen zu lernen. 
Heike Henning: Es gilt, zwei Perspektiven abzugrenzen, zum einen ist praxisbezogen zu fragen, wie man Musikunterricht inklusiv gestaltet, zum anderen ist aus gesellschafts- und schulpolitischer Perspektive zu fragen, welchen Beitrag Musikunterricht leisten könnte, um Gesellschaft inklusiv zu gestalten.

Julia Lutz: Ich sehe für die Musikpädagogik, die sich mit schulischem Unterricht beschäftigt, die praktische Frage als vorherrschend an. Mittlerweile gibt es für die Gestaltung eines inklusiven Musikunterrichts zahlreiche Veröffentlichungen, die methodische Anhaltspunkte geben. Mir ist es ganz wichtig, den zweiten Punkt zu betonen, der viel zu wenig berücksichtigt wird. Wie kann ich Schulleben und -alltag inklusiv gestalten und wie kann ich dies mit Musik tun? Wie kann ich durch Begrüßungslieder, Bodypercussion etc. in allen Fächern über gemeinsames musikalisches Erleben Inklusion schaffen. Das scheint insbesondere für viele Grundschullehrende ein ganz wichtiger Ansatzpunkt zu sein. In dem genannten Kurs stellen wir fest, dass es ein toller Ansatzpunkt ist, die Studierenden über die Inklusion offen für Musik zu machen und sie dazu anzuregen, viel mehr Musik in ihren Schulalltag einzubauen.

Heike Henning: Frau Salmon, Sie geben Menschen in verschiedenen Lehrgängen bspw. Musik und Tanz in sozialer Arbeit und inklusiver Pädagogik Handwerkszeug für dieses inklusive Lehren und Lernen an die Hand. Was leisten Musik und Tanz in der inklusiven Pädagogik?

Shirley Salmon: Ich würde das gar nicht trennen. Eine gute Pädagogik müsste vom Denken her sowieso inklusiv sein. Das sind genauso musikalische oder tänzerische Ziele je nach Potenzial der Jugendlichen, Kinder oder Erwachsenen und natürlich gibt es auch diese sozialen Aspekte. Ich denke in der Pädagogik ist es gut, wenn man nicht sagt Musik und Tanz mit Menschen mit Behinderung hat gleich therapeutische Zwecke. Es gibt vielleicht therapeutische Nebeneffekte, aber es ist nicht die Aufgabe der Pädagogik zu therapieren. Diese Fehleinschätzung haben wir früher öfter bei Leuten gesehen, die bei uns diesen Studienschwerpunkt belegten. Aber dazu braucht man eine zusätzliche Ausbildung und Pädagogik ist nicht gleich Therapie. Studierende, egal ob an den pädagogischen Hochschulen oder bei uns am Orff-Institut, müssen zuerst mal diese Haltung haben, diese Ressourcen, dieses Potenzial von Vielfalt zu sehen. Wenn ich diese Haltung nicht habe, werde ich nie gut mit heterogenen Gruppen arbeiten können. Und ein zweiter Aspekt ist die Beziehungsebene. Ich muss in der Lage sein, auf verschiedenen Ebenen in Beziehung gehen zu wollen. Das ist fundamental. Wenn ich nur etwas Musikalisches oder Tänzerisches erreichen will, erreiche ich nicht die Menschen.

Heike Henning: Wieviel Therapeutisches steckt in Musikpädagogik? Sind persönlichkeitsbildende Ziele, die wir durchaus legitim im Unterricht verfolgen, nicht 
immer auch therapeutisch, wenn sie uns näher zu uns selbst, zu unseren Mitmenschen, Potenzialen und Idealen bringen?

Shirley Salmon: Doch, diese Anteile sind in der Praxis da. Dennoch bilden wir keine Therapeut(*inn)en aus. Therapie und Pädagogik zu vermischen ist gefährlich. In der Ausbildung ist es ganz wichtig zu klären, was unter Pädagogik mit Menschen mit unterschiedlichen Fähigkeiten zu verstehen ist. Persönlichkeitsbildende Ziele sind grundsätzliche, humanistische Aspekte, die Teil jeder Pädagogik sein müssten. Das ist für mich nicht Therapie, wenn ich Musik und Tanz in einem inklusiven Kontext anbiete.

Heike Henning: Die Abgrenzung ist jedoch nicht immer leicht, weil Musik auch therapeutische Wirkungen hat - egal ob intendiert oder nicht.

Robert Wagner: Wir müssen einen deutlichen Unterschied herausarbeiten: Es gibt in Deutschland einen berufsbegleitenden Lehrgang Instrumentalmusik mit Menschen mit Behinderung. Hier liegt der Schwerpunkt ganz klar auf dem pädagogischen und nicht auf dem therapeutischen Aspekt. Wir dürfen uns nicht anmaßen therapeutisch zu handeln, denn wir haben die medizinische Ausbildung nicht. Der große Unterschied zwischen Therapie und Pädagogik ist: Beides ist absichtsvolles Handeln, aber bei der Therapie geht's um ein bekanntes Defizit, das behoben oder gemildert werden muss. Es gibt ein klares Setting, eine klare Zeitvorgabe und man vereinbart mit der zu therapierenden Person einen zeitlichen Umfang. Nach der gemeinsamen Arbeit schaut man, ob sich etwas gebessert hat etc. Pädagogik ist etwas ganz anderes. Früher war es ganz klar, dass es um einen Aufbau von Kompetenz geht. Heute wird immer klarer, dass es auch um einen ganz normalen Abbau an Fähigkeiten und Kompetenzen gehen kann. Musikpädagog( ${ }^{*}$ inn)en begleiten würdevoll nach oben und nach unten, denn Fertigkeiten des Menschen nehmen eben nicht nur zu, sondern auch ab.

Renate Reitinger: In der Pädagogik gibt es leider manchmal eine gefährliche Haltung, die zu stark auf Defizite gerichtet ist. Diese Sicht gilt es zu verändern. Natürlich ist es leichter zu sehen, was jemand nicht kann als was er kann. Aber Letzteres sollte in den Blick genommen werden, nicht die Defizite, was dann therapeutisch wäre.

Günther Klausner: Ich glaube, dass dies auch ein Punkt ist, der im Zusammenhang mit Inklusion viele Ängste in unserem Kollegium schürt. Deshalb sagen manche, dass sie mit einem Menschen mit Förderbedarf nicht arbeiten können, weil sie hierfür nicht ausgebildet sind. Und dabei haben sie dann nicht mehr das Pädagogische, sondern das Therapeutische im Blick. Das können wir nicht und das sollen wir auch nicht. 
Julia Lutz: Ich möchte die Abgrenzung von Therapie und Pädagogik noch einmal unterstützen mit einem Blick in die Förderschulen, die es in Deutschland noch gibt. Dort wird ganz klar unterschieden zwischen Musikunterricht und Musiktherapie.

Michael Turinsky: Ich wollte noch einmal einhaken, bei dem, was schon mehrmals gesagt wurde, wie wichtig es ist, Potenzial zu erkennen und würde gerne einen Bogen spannen zum Anfang unserer Diskussion. Da haben Sie, Frau Henning, uns gefragt, was wir denn unter Qualität verstehen. Ich denke, dass es sehr wichtig ist, über Strukturen usw. zu reden, aber eben auch über Haltungen. Ein wesentlicher Aspekt besteht meines Erachtens darin, eine kritische Haltung zu jedweder Qualität zu entwickeln. Das heißt nun sicherlich nicht, den Begriff Qualität zu negieren. Wir müssen fähig werden, zu begründen, warum wir ein (inklusives) Projekt für wichtig halten und nicht irgendwelche etablierten Qualitätsstandards übernehmen. Und eben nicht alle Künstler*innen über einen Kamm scheren und sie an dieses Qualitätsverständnis anzupassen. Vielmehr müssen wir lernen, sehr breit und innovativ über Qualität nachzudenken. Ich denke, gute Kunst definiert immer wieder selbst ihre Qualität neu, beispielsweise Zeitgenössische Kunst, und genau das macht sie interessant. Und ich würde mir wünschen, dass dieses kritische Denken auch beim Unterricht mit behinderten Menschen gefördert wird - das kritische Nachdenken darüber, welche Qualität angestrebt wird. Diese kritische Auseinandersetzung mit dem Qualitätsbegriff vermisse ich.

Heike Henning: Wir könnten noch über so vieles sprechen, Gesagtes vertiefen. Leider ist die Zeit um. Ich danke Ihnen für diesen spannenden Diskurs. 



\section{Einschätzung von Beziehungsqualität als Grundpfeiler inklusiven Arbeitens}

\section{Das EBQ-Instrument in der Praxis}

"Jeder Mensch braucht einen Menschen, um sich seinen Anlagen entsprechend zu entwickeln [...] Denn ohne die Erfahrung einer zwischenmenschlichen Begegnung kann die Fähigkeit, sich auf jemanden und etwas zu beziehen, nicht entwickelt werden." (Schumacher, 2017, S. 13)

Der Begriff Inklusion und die damit verbundenen Modelle einer pädagogischen Herangehensweise sind auf dem Weg zu einer Zentralthematik für die kommenden Jahre zu werden. Als Querschnittsmaterie - entwickelt aus der menschenrechtlich basierten Idee - ist Inklusion in den unterschiedlichsten Lebensbereichen bedeutsam geworden (vgl. Hedderich, Biewer, Hollenweger \& Markowetz, 2016; Heimlich, 2012; Lütje-Klose, Langer, Serke \& Urban, 2011). Auch für den Bereich der Musik- und Tanzpädagogik entstanden in den letzten Jahren verschiedene Ansätze und Publikationen, die sich im Besonderen der Thematik der Inklusion widmen (vgl. Jank \& Bossen, 2017; Keller, 1996; Salmon, 1999, 2006, 2016; Verband deutscher Musikschulen, 2017; Wagner, 2002).

Neben der Bedeutung, die didaktische Modelle und praxisbezogene Empfehlungen hier einnehmen (vgl. Eberhard, Hirte \& Höfer, 2017; Neikes, 1998; Peer \& Reid, 2012; Tscheke, 2015; Wagner, 2016), soll in diesem Beitrag auf Vorbedingungen für inklusives pädagogisches Arbeiten eingegangen werden.

Beziehungen sind die Grundlage für die menschliche Entwicklung und sollten vor allem in pädagogischen Kontexten einer genaueren Betrachtung unterzogen werden, da sie als Grundpfeiler dienen, an dem die Ausgestaltung der Lehr- und Lernprozesse orientiert wird. Musik- und Bewegungserziehung bietet eine ideale Basis für einen ganzheitlichen Ansatz, der auf Beziehung beruht, indem sie kognitive, emotionale, soziale und kreative Bereiche des Menschen anspricht. 


\section{Beziehungsfähigkeit}

Die Bedeutung des Beziehungsaspektes im Kontext pädagogischer Angebote wurde in den letzten Jahren ausführlich dargestellt (vgl. Cornelius-White, 2007; Hattie, 2015; Knierim, Raufelder \& Wettstein, 2016; Winnerling, 2005). In der inklusiven Pädagogik ist jedoch noch ein vertiefender Blick notwendig, der die grundlegende Dimension der Beziehungsfähigkeit detaillierter betrachtet.

Joachim Bauer beschreibt den Begriff der Beziehungskompetenz und charakterisiert diesen mittels fünf Komponenten:

1. Sehen und gesehen werden. Menschen möchten in erster Linie als Personen wahrgenommen werden.

2. Gemeinsame Aufmerksamkeit gegenüber etwas Drittem. Dies wird auch als ,Joint Attention' bezeichnet.

3. Emotionale Resonanz. Die Fähigkeit, sich auf die emotionale Befindlichkeit eines anderen einzustellen.

4. Gemeinsames Handeln. Das gemeinsame Tun hat beziehungserzeugende Effekte.

5. Wechselseitiges Verstehen von Motiven und Absichten. Wenn die vier vorangehenden Elemente bereits vorhanden sind, lassen sich Motive, Absichten und Ähnliches von anderen Menschen erkennen und neue Möglichkeiten für Beziehung entfalten.

(vgl. Bauer, 2014, S. 193-195)

Die Beziehungskompetenz wird grundgelegt und entscheidend beeinflusst durch die Entwicklung der Bindungsfähigkeit in einem typischen Verlauf in den ersten beiden Lebensjahren. Ist diese Entwicklung durch unterschiedlichste Faktoren beeinträchtigt, können Komponenten der Beziehungskompetenz entweder unvollständig oder gar nicht ausgebildet werden (vgl. Fuhrer, 2009; Stephan, 2015; vgl. auch Zitat Schumacher, 2017 am Beginn des Beitrags). Für ein inklusives Unterrichtsmodell sind diese jedoch von großer Bedeutung, da die Beziehungsmöglichkeiten Grundlage jeglichen Lernens bilden.

Umso mehr überrascht es, dass eine systematische Auseinandersetzung mit den unterschiedlichen Beziehungskompetenzen bzw. Beziehungsfähigkeiten in pädagogischen Kontexten selten vorzufinden ist. Daher erscheint die genaue Beobachtung und Analyse der Beziehungsmöglichkeiten der beteiligten Personen wichtig, denn erst wenn die Beziehungsmöglichkeiten und somit auch die Aufnahmebereitschaft der Kinder erfasst wurden, ist ein entsprechendes Angebot möglich. Inklusive Lernkontexte in heterogenen Gruppen gelingen vor allem dann, wenn die unterschiedlichen Fähigkeiten und Bedürfnisse der Kin- 
der ${ }^{1}$ und ihre Beziehungsfähigkeit richtig eingeschätzt werden und Lehrende ihre Angebote darauf aufbauend möglichst individuell an die Kinder anpassen.

Eine derartige Herangehensweise zur Beobachtung und Analyse der Beziehungsqualität wurde mit dem EBQ-Instrument von Schumacher, Calvet und Reimer (2013) vorgestellt, welches ursprünglich für den Bereich der Musiktherapie entwickelt wurde. Eine Adaption dieses Verfahrens für die Nutzung im pädagogischen Feld wird derzeit am Orff-Institut der Universität Mozarteum Salzburg erarbeitet (vgl. Esterbauer, 2018, 2019; Esterbauer, Salmon \& Schumacher, 2018) $)^{2}$, da aufgrund unterschiedlicher Zielsetzungen, Methoden und Settings, aber auch aufgrund andersgearteter Voraussetzungen eine Anpassung des Verfahrens notwendig erscheint.

\section{Das EBQ-Instrument}

Das von Karin Schumacher und Claudine Calvet seit 1990 entwickelte EBQInstrument (kurz: EBQ) ist ein qualitatives Beobachtungsverfahren und dient der Einschätzung der Beziehungsqualität. „Das EBQ-Instrument fokussiert die Art und Weise, wie Beziehung zu sich (Körper und Stimme), zu Objekten (Musikinstrumente) und zum Anderen (Musiktherapeut) aufgenommen wird. Mit Hilfe bestimmter Merkmale wird die Qualität dieser Beziehungsfähigkeit eingeschätzt und damit möglichst nachvollziehbar bestimmt." (Schumacher, Calvet \& Reimer, 2013, S. 36)

Vor allem das Selbstkonzept des Säuglingsforschers Daniel N. Stern (1985) war Ausgangspunkt für die Entwicklung dieses Verfahrens, welches sich in seiner Struktur am Schichtenmodell Sterns orientiert. Ergänzende theoretische Impulse wurden der Bindungsforschung (vgl. Bowlby, 1988; Grossmann \& Grossmann, 2012) sowie der Hirnforschung (vgl. Hüther, 2004) entnommen. Daraus entwickelten Schumacher und Calvet eine entwicklungspsychologische Sicht vor allem auf das Störungsbild Autismus.

1 Im weiteren Text wird meist vom Kind als Adressat*in der therapeutischen bzw. auch pädagogischen Interventionen gesprochen. Diese können sich aber natürlich auch auf erwachsene Personen beziehen.

2 Siehe dazu: Esterbauer (2019) bzw. https://www.uni-mozarteum.at/apps/app_ck/ ckuserfiles/67428/files/Information\%20EBQ-Forschungsprojekt\%20(Stand\%20 Juni\%202017).pdf [05.10.2020]. 


\section{Beobachtungsschwerpunkte}

Das EBQ-Instrument umfasst vier Skalen, die mit Beobachtungsschwerpunkten arbeiten.

Diese Beobachtungsschwerpunkte beschreiben Merkmalskomplexe, anhand derer die Qualität von Beziehungsfähigkeit eingeschätzt werden kann. So werden bei der Analyse des körperlich-emotionalen Ausdrucks (KEBQ) die intra- und interpersonelle Beziehung, der Körperkontakt, der Affekt und die Qualität des Blickkontaktes beobachtet. Die Skala des stimmlich-vorsprachlichen Ausdrucks (VBQ) bezieht sich auf die Ausdrucksqualität der Stimme, ihre Beziehung zum eigenen Körper und zumzur TherapeutenTherapeutin sowie die stimmlich-vorsprachlichen Mittel im Hinblick auf das entsprechende Entwicklungsalter. Bei der Einschätzung des instrumentalen Ausdrucks (IBQ) werden die Wahl des Musikinstrumentes, der Objektbezug, die verwendeten musikalischen Mittel und der Spielraum betrachtet, der auf und mit dem Musikinstrument entfaltet wird (vgl. Schumacher et al., 2013). Als Grundlage der Einschätzung der Beziehungsqualität fungieren entwicklungspsychologisch orientierte Merkmallisten zu jeder dieser vier Skalen. Von zentraler Bedeutung aber ist die Skala zur Einschätzung der $^{\star}$ des Therapeutin ${ }^{\star} T h e r a p e u t e n$ (TBQ) und ihrer*seiner Interventionen, welche die Effektivität der musiktherapeutischen Arbeit zu überprüfen hilft: „Aus entwicklungspsychologischer Sicht ist es wichtig, ganz vom kindlichen Entwicklungsstand ausgehend und entsprechend seiner Ausdrucksmöglichkeiten zu intervenieren. Durch die Einschätzung mit Hilfe der TBQ-Skala können mögliche Interventionsfehler, sowie Über- und Unterstimulation aufgedeckt werden." (Esterbauer et al., 2018, S. 20)

\section{Die sieben Modi}

Für jede der Skalen wurden sieben Beziehungsqualitäten beschrieben, die als Modi bezeichnet wurden (vgl. Schumacher et al., 2013). ${ }^{3}$ Die Reihenfolge der Modi 0 bis 6, die im Allgemeinen eine Zunahme von Beziehungsqualität abbildet $^{4}$, ist in jeder der vier Skalen gleich und basiert auf den sozioemotionalen Entwicklungskonzepten von Stern $(2000,2007)$ und Sroufe (1997).

\section{Modus 0: Kontaktlosigkeit/Kontaktabwehr}

Das Hauptmerkmal ist Kontaktlosigkeit. Die Angebote des ${ }^{\star}$ der Therapierenden werden vom Kind scheinbar nicht wahrgenommen oder abgelehnt. Das Kind

3 Modus bedeutet in diesem Zusammenhang die Art und Weise, in der die Beziehung ausgedrückt wird.

4 Die Zahlen der Modi von 0 bis 6 beziehen sich auf den Umfang der Beziehungsfähigkeit, beginnend bei 0 mit keinem Kontakt bis zu 6 mit interaffektivem Austausch. 


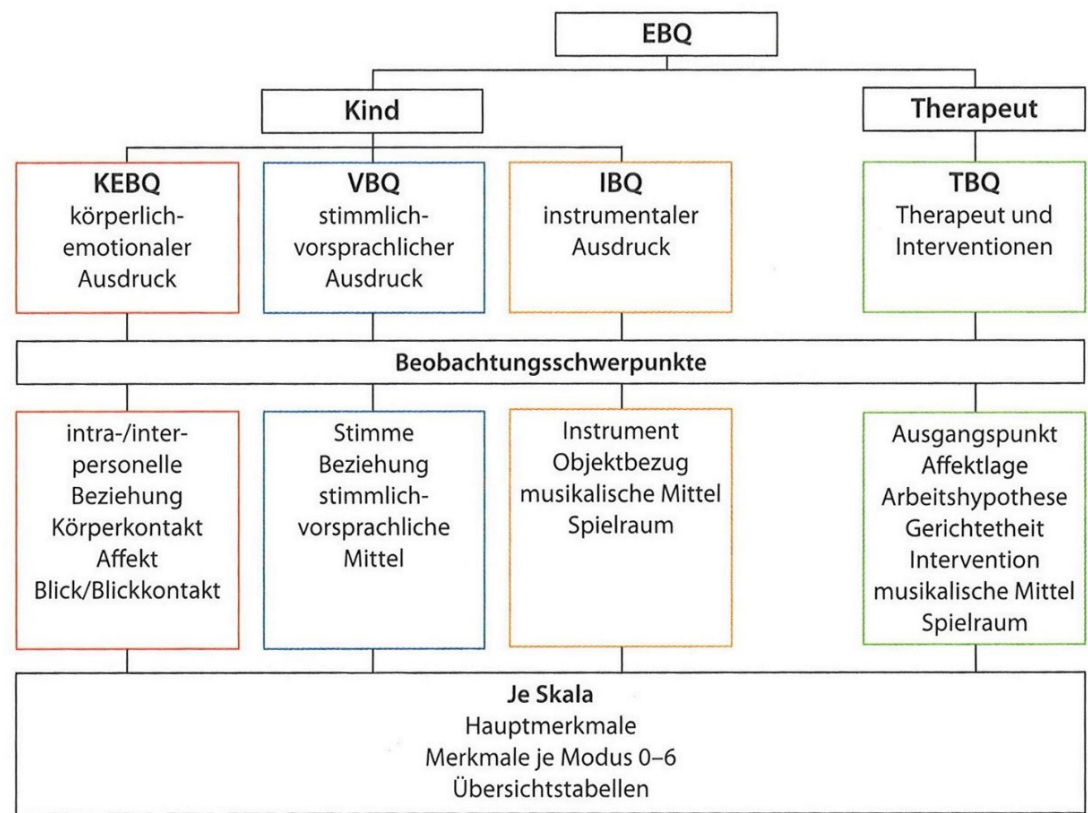

Abbildung 1: Struktur des EBQ-Instruments (aus Schumacher et al., 2013, S. 36)

ist in seinem stereotypen Verhalten gefangen. Die musikalische Intervention soll eine Atmosphäre schaffen, die eine Beziehung ermöglicht, diese aber nicht fordert.

\section{Modus 1: Sensorischer Kontakt/Kontakt-Reaktion}

Der ${ }^{\star}$ Die Therapeut ${ }^{\star}$ in konzentriert sich auf die sensorischen Bedürfnisse und/ oder die stereotypen Bewegungen des Kindes. Gelingt die Verknüpfung der durch die Therapie angebotenen koordinierten Reize, führt das Erleben synchroner Momente zu kurzen positiven Kontaktreaktionen.

\section{Modus 2: Funktionalisierender Kontakt}

Der körperlich-emotionale Ausdruck des Kindes zeigt eine hohe Anspannung und der affektive Ausdruck steht im Vordergrund. Instrumente werden oft in destruktiver Weise verwendet. Der ${ }^{\star}$ Die Therapierende reguliert hohe Affekte durch Affektabstimmung und musikalische Affektgestaltung. Das Kind soll erleben, dass Affekte durch die Hilfe einer*eines Anderen reguliert werden können. 


\section{Modus 3: Kontakt zu sich selbst/Selbsterleben}

Das Kind ist aufmerksam und ruhig während es seinen eigenen Körper, Musikinstrumente bzw. seine Stimme erkundet. Es erlebt sich als selbstwirksam und als Urheber*in einer Handlung. Die ${ }^{\star}$ Der Therapierende unterstützt diese Exploration indem die Äußerungen und Handlungen des Kindes imitiert, begleitet, umspielt und musikalisch eingebettet werden.

\section{Modus 4: Kontakt zurł zum Anderen/Intersubjektivität}

Das Kind zeigt Interesse an der gemeinsamen Aktivität und an der*dem Therapierenden. Die Fähigkeit zur gemeinsamen Aufmerksamkeit (Joint Attention) führt oft zu einem Blickaustausch. Die*Der Therapierende entspricht dem Bedürfnis des Kindes nach sozialer Rückversicherung und kann eigene musikalische Ideen einbringen, ohne einen Dialog mit dem Kind zu erwarten.

\section{Modus 5: Beziehung zurł zum Anderen/Interaktivität}

Hauptmerkmal ist die Fähigkeit zu imitieren. Im dialogischen Spiel werden der wechselseitige Austausch von Motiven, das aufeinander Bezugnehmen sowie das Einhalten von Wechselpausen deutlich.

\section{Modus 6: Begegnung/Interaffektivität}

Die Beziehung zwischen $\operatorname{der}^{*}$ dem Therapierenden und dem Kind ist etabliert und das gemeinsame ausdrucksvolle Spiel geht mit einer positiven emotionalen Befindlichkeit beider Spieler*innen einher. Kind und Therapeut*in erleben gemeinsame Freude am Spiel.

\section{Durchführung}

Bei der Arbeit mit dem EBQ-Instrument wird eine Therapiestunde per Video aufgenommen. Je nach Fragestellung wird daraus eine Sequenz von maximal drei Minuten ausgewählt. Zu Beginn steht die Frage, welche Intervention die ${ }^{\star}$ der Therapierende anwendet. Dies kann mit Hilfe der TBQ-Skala beurteilt werden. Je nachdem, ob sich das Kind körperlich-emotional, vokal oder instrumental ausdrückt, wird die Szene mit einer oder mehreren der drei Skalen analysiert. Nur wenn der Modus der des Therapierenden mit dem des Kindes in Übereinstimmung ist, kann ein konstruktiver Therapieverlauf erwartet werden (vgl. Mössler et al., 2017). Wenn die Modi nicht kongruieren, werden das Vorgehen der $^{*}$ des Therapierenden die Arbeitshypothesen und Zielsetzungen sowie die musikalischen Mittel diskutiert. Dabei ist es von besonderer Wichtigkeit, Interventionsfehler erkennen und beheben zu können. ${ }^{5}$

5 Das EBQ-Instrument kommt im Regelfall in einem therapeutischen Prozess zum Einsatz. Die Einschätzung wird dabei von speziell ausgebildeten Personen durchgeführt. Dies kann ein ${ }^{\star}$ Supervisor ${ }^{\star}$ in, ein ${ }^{\star}$ S Forscher ${ }^{\star}$ in etc. aber auch $\operatorname{die}^{\star}$ der Therapierende selbst sein. 


\section{Anwendung des EBQ-Instruments in der Musik- und Tanzpädagogik}

Wie bereits erwähnt wurde das EBQ-Instrument für den musiktherapeutischen Bereich und hier vor allem für die Arbeit mit tiefgreifend entwicklungsgestörten Kindern - speziell mit Autismus - entwickelt. Daher besteht die Notwendigkeit, dieses für jeden anderen Anwendungsbereich zu adaptieren. ${ }^{6}$ Im Bereich der Pädagogik sind ebenso bereits Anwendungen durchgeführt worden, wie z. B. speziell für inklusive Kontexte von Fragkouli (2012) und Salmon (2012). Auch eine Sprachskala, durch welche die Beziehungsqualität verbaler Äußerungen eingeschätzt werden soll und die für den pädagogischen Bereich besondere Bedeutung hat, wurde entwickelt (vgl. Jordan, 2016, 2017). Insbesondere durch das Forschungsprojekt Einschätzung von Beziehungsqualität im Musikunterricht in inklusivem Setting der Universität Mozarteum Salzburg (Orff-Institut) wird eine Adaption des EBQ für den musik- und tanzpädagogischen Gebrauch erarbeitet (vgl. Esterbauer, 2018, 2019; Esterbauer et al., 2018). Diesbezügliche Änderungen bzw. Erweiterungen des EBQ betreffen zum einen die Einschätzung des Entwicklungsniveaus und der Beziehungsfähigkeit der Kinder und zum anderen auch die pädagogischen Interventionen. Dabei sollen im Speziellen folgende drei Fragen behandelt werden:

1) Welche der eingesetzten Interventionen bzw. Arbeitsmethoden zeigen welche Wirkung auf die Beziehungsqualität und das Verhalten von Kindern im inklusiven Setting?

2) Welche der beschriebenen Interventionen und Methoden führen zu einer Zunahme von Beziehungsfähigkeit und Beziehungsbereitschaft?

3) Welche spezifischen Vorgehensweisen und pädagogischen Interventionen erweisen sich als vorteilhaft bzw. förderlich?

Im Fokus der Anwendung des EBQ-Instruments für den inklusiven Musikund Tanzunterricht stehen vor allem jene Kinder, die weniger gut erreichbar, motivierbar oder gruppenfähig sind, also jene, die eine spezielle Zeitgestaltung oder didaktische Herangehensweise benötigen. Ziel ist es, für diese Kinder geeignete Interventionen $\mathrm{zu}$ finden, um ihnen (und auch den anderen) eine positive Teilnahme am Gesamtgeschehen zu ermöglichen. Durch das EBQ könnte in Folge ein Handlungsleitfaden für die pädagogische Praxis entwickelt werden, der aufzeigt, welche Beziehungsqualitäten wie gestärkt bzw. verändert werden können und für welches Entwicklungsniveau der Kinder welche Ange-

6 Für u.a. folgende Anwendungsbereiche wurden EBQ-Analysen publiziert: Neurologie, Psychosomatik, Psychiatrie, Demenz, Erwachsene mit schwerster Mehrfachbehinderung. 
bote am geeignetsten sind. Vor allem die Fähigkeit zu Joint Attention, also das gemeinsame Fokussieren auf etwas Drittes (vgl. Moore \& Dunham, 1995; Seemann, 2011) ist im pädagogischen Alltag eine wichtige Grundvoraussetzung, die mit Hilfe des adaptierten EBQ beobachtet werden kann und auf die in der pädagogischen Arbeit Augenmerk gelegt werden sollte (vgl. Esterbauer et al., 2018).

Ausgehend von Ergebnissen aus der Musiktherapie, wonach eine wirksame Beziehung dann eintritt, wenn musikalisch und emotional auf die kindlichen Äußerungen eingegangen wird und vom aktuellen Entwicklungsstand des Kindes ausgehend weiterführende Spielideen entwickelt werden (vgl. Mössler et al., 2017), ist in ähnlicher Weise anzunehmen, dass auch für eine pädagogische Beziehung im inklusiven Setting diese Übereinstimmung der Beziehungsqualitäten ${ }^{7}$, wie sie im EBQ beobachtbar sind, von immenser Bedeutung ist (vgl. Esterbauer et al., 2018).

\section{Vorteile des EBQ-Instruments für die Elementare Musik- und Tanzpädagogik}

Welche Vorteile bietet also die Verwendung des EBQ in der inklusiv-pädagogischen Praxis und insbesondere in der Elementaren Musik- und Tanzpädagogik?

In erster Linie ermöglicht das EBQ eine Einschätzung der Beziehungsfähigkeit des Kindes und der darauf abzustimmenden pädagogischen Beziehungsqualität, also der Kontaktaufnahme sowie der beziehungsmäßigen Gestaltung der sozialen, emotionalen und kognitiven Inhalte der Lern-, Erfahrungs- oder Erlebniseinheiten. Durch diese Einschätzung wird in Folge die Auswahl adäquater Inhalte musik- bzw. tanzpädagogischer Aktivitäten für die unterschiedlichen Fähigkeiten und (Lern-)Bedürfnisse wesentlich erleichtert, da das Erkennen von Über- und Unterforderung ermöglicht wird und somit Frustrationen im pädagogischen Prozess vermieden werden können. Fordert die Lehrperson zu schnell zum Mit- oder Nachmachen oder zum Zusammenspiel auf, so kann dies für einige Kinder eine Überforderung darstellen und die Partizipationsbereitschaft verringern. Ein weiterer wichtiger Bestandteil der Arbeit mit dem EBQ-Instrument ist eine kritische Reflexion der Arbeit der jeweiligen Lehrperson auf den unterschiedlichsten Ebenen wie Inhalt, Didaktik, Emotionen, Sozialverhalten, Wahrnehmung, Interpretation kindlichen Verhaltens etc.

7 Eine Übereinstimmung der Beziehungsqualitäten lässt sich im EBQ dadurch nachweisen, dass sowohl Therapeut*in als auch Kind bzw. Lehrperson und Schüler*in ein beobachtbares Verhalten im gleichen Beziehungsmodus zeigen. 
Das EBQ-Instrument erweist sich als ein geeignetes Verfahren zur Beobachtung und Einschätzung der Beziehungsqualität und kann im Bereich der inklusiven Musik- und Tanzpädagogik einen ergänzenden Beitrag leisten, um einen den Möglichkeiten und Fähigkeiten aller Kinder entsprechenden Unterricht gestalten zu können, damit ihre Entwicklung auf verschiedensten Ebenen ganzheitlich unterstützt werden kann.

\section{Literatur}

Bauer, J. (2014). Prinzip Menschlichkeit. Warum wir von Natur aus kooperieren (7. Aufl.). München: Wilhelm Heyne Verlag.

Bowlby, J. (1988). A secure base. Clinical applications of attachement theory. London: Routledge.

Cornelius-White, J. (2007). Learner-Centered Teacher-Student Relationships Are Effective. A Meta-Analysis. Review of Educational Research, 77 (1), 113-143. Verfügbar unter: https://doi.org/10.3102/003465430298563 [30.06.2020]. https:// doi.org/10.3102/003465430298563

Eberhard, D. M., Hirte, G. \& Höfer, U. (2017). Inklusions-Material Musik, Klasse 1-4. Berlin: Cornelsen.

Esterbauer, E. (2019). Assessment of the quality of relationship in music lessons - a new approach. Joint (ad)venture of music therapy and music pedagogy. In M. Oebelsberger, A. Bernhofer \& G. Sammer (Hrsg.), Joint (Ad)Venture (European Perspectives on Music Education, Bd. 8, S. 123-134). Innsbruck: Helbling.

Esterbauer, E. (2018). Relationship in special music education - a new form of assessment. The AQR-Tool in inclusive music lessons. In G. Tsiris \& K. VanWeelden (Hrsg.), Music Across the Lifespan: The Role of Special Music Education and Music Therapy. Proceedings of the 22nd International Seminar of the ISME Commission on Special Music Education and Music Therapy (S. 52-64). o.O.: ISME.

Esterbauer, E., Salmon, S. \& Schumacher, K. (2018). Einschätzung der Beziehungsfähigkeit mit Hilfe des „EBQ-Instruments“ im inklusiven Musikunterricht. Diskussion Musikpädagogik, 20 (79), 18-23.

Fragkouli, A. (2012). Music therapy for children with psychological disorders in special education. Dissertation, Nationale und Kapodistrias-Universität Athen.

Fuhrer, U. (2009). Lehrbuch Erziehungspsychologie (2. überarb. Aufl.). Göttingen: Verlag Hans Huber.

Grossmann, K. \& Grossmann, K. E. (2012). Bindungen. Das Gefüge psychischer Sicherheit (5. vollst. überarb. Aufl.). Stuttgart: Klett-Cotta.

Hattie, J. (2015). Lernen sichtbar machen (3. erweiterte Aufl.). Baltmannsweiler: Schneider-Verlag Hohengehren.

Hedderich, I., Biewer, G., Hollenweger, J. \& Markowetz, R. (Hrsg.). (2016). Handbuch Inklusion und Sonderpädagogik (utb Erziehungswissenschaft, Sonderpädagogik). Bad Heilbrunn: Klinkhardt. 
Heimlich, U. (2012). Inklusion in Schule und Unterricht. Wege zur Bildung für alle (Praxis Heilpädagogik: Handlungsfelder). Stuttgart: Kohlhammer.

Hüther, G. (2004). Ebenen salutogenetischer Wirkungen von Musik auf das Gehirn. Musiktherapeutische Umschau, 25 (1), 16-26.

Jank, B. \& Bossen, A. (Hrsg.). (2017). Musikarbeit im Kontext von Inklusion und Integration. Potsdamer Schriftenreihe zur Musikpädagogik. Potsdam: Universitätsverlag Potsdam.

Jordan, A.-K. (2016). Weiterentwicklung der Skalen zur Einschätzung der Beziehungsqualität im musiktherapeutischen Setting mit Fokus auf den sprachlichen Ausdruck. Analyse von Audio- und Videosequenzen einer musiktherapeutischen Langzeitbehandlung eines Jungen mit Autismus-Spektrums-Störung. Masterarbeit, Universität der Künste Berlin.

Jordan, A.-K. (2017). Assessing the Quality of Relationship in Music Therapy Sessions with the Focus on Language. Spoken paper at the ESCOM conference, Gent, Belgien.

Keller, W. (1996). Musikalische Lebenshilfe. Ausgewählte Berichte über sozial- und heilpädagogische Versuche mit dem Orff-Schulwerk. Herausgegeben von Hermann Regner und Klaus W. Oberborbeck. Mainz [u.a.]: Schott.

Knierim, B., Raufelder, D. \& Wettstein, A. (2016). Die Lehrer-Schüler-Beziehung im Spannungsfeld verschiedener Theorieansätze. Psychologie in Erziehung und Unterricht, 84 (1), 35-48. https://doi.org/10.2378/peu2017.art04d

Lütje-Klose, B., Langer, M.-T., Serke, B. \& Urban, M. (Hrsg.). (2011). Inklusion in Bildungsinstitutionen. Eine Herausforderung an die Heil-und Sonderpädagogik. Bad Heilbrunn: Klinkhardt.

Moore, C. \& Dunham, P. J. (Hrsg.). (1995). Joint attention. Its origins and role in development. Hillsdale, NJ: Lawrence Erlbaum Associates.

Mössler, K., Gold, C., Aßmus, J., Schumacher, K., Calvet, C., Reimer, S., Iversen, G. \& Schmid, W. (2017). The Therapeutic Relationship as Predictor of Change in Music Therapy with Young Children with Autism Spectrum Disorder. Journal of autism and developmental disorders, 47 (Sep 21), 1-15.

Neikes, J. L. (1998). Scheiblauer-Rhythmik. Überarbeitet und neu herausgegeben von Eliosabeth Danuser-Zogg (5. Aufl.). Sankt Augustin: Academia-Verl.

Peer, L. \& Reid, G. (Hrsg.). (2012). Special educational needs. A guide for inclusive practice. London: Sage.

Salmon, S. (1999). Behinderung als Herausforderung. Orff-Schulwerk Informationen (62), 11-19. Verfügbar unter http://bidok.uibk.ac.at/library/salmon-herausfor derung.html [05.10.2020].

Salmon, S. (Hrsg.). (2006). Hören, Spüren, Spielen. Musik und Bewegung mit schwerhörigen und gehörlosen Kindern (Zeitpunkt Musik). Wiesbaden: Reichert.

Salmon, S. (2012). Die Einschätzung der Beziehungsqualität bei Menschen mit Schwerstbehinderung in einem musikpädagogischen Setting. In G. Enser \& M. Oebelsberger (Hrsg.), Vernetzung als Chance für die Musikpädagogik (Musikpä- 
dagogische Forschung Österreich: Reihe 1: Tagungsberichte, Bd. 4, S. 133-148). Wien: Universal Edition.

Salmon, S. (2016). How the Orff Approach Can Support Inclusive Teaching. In D. VanderLinde Blair \& K. A. McCord (Hrsg.), Exceptional Pedagogy for Children with Exceptionalities: International Perspectives (S. 39-60). Oxford: Oxford University Press. https://doi.org/10.1093/acprof:oso/9780190234560.003.0003

Schumacher, K. (2017). Musiktherapie bei Kindern mit Autismus. Musik-, Bewegungs- und Sprachspiele zur Behandlung gestörter Sinnes- und Körperwahrnehmung (mit DVD zum EBQ-Instrument). Wiesbaden: Reichert.

Schumacher, K., Calvet, C. \& Reimer, S. (2013). Das EBQ-Instrument und seine entwicklungspsychologischen Grundlagen (2. Aufl.). Mit 10 Abb., 5 Tab. und einer DVD mit 28 Praxisbeispielen. Göttingen: Vandenhoeck \& Ruprecht.

Seemann, A. (Hrsg.). (2011). Joint attention. New developments in psychology, philosophy of mind, and social neuroscience. Cambridge, MA: MIT Press. https://doi. org/10.7551/mitpress/8841.001.0001

Sroufe, L. A. (1997). Emotional development. Cambridge: Cambridge University Press.

Stephan, C. (2015). Bindungsbeziehung - Spielbeziehung - Kompetenzentwicklung. In G. Spangler \& P. Zimmermann (Hrsg.), Die Bindungstheorie. Grundlagen, Forschung und Anwendung (7. Auflage, S. 265-280). Stuttgart: Klett-Cotta.

Stern, D. N. (1985). The interpersonal world of the infant. A view from psychoanalysis and developmental psychology. New York: Basic Books.

Stern, D. N. (2000). The interpersonal world of the infant. A view from psychoanalysis and developmental psychology (1st paperback ed.). New introduction by the author. New York: Basic Books.

Stern, D. N. (2007). Die Lebenserfahrung des Säuglings (9. erweiterte Aufl.). Mit einer neuen Einleitung des Autors. Stuttgart: Klett-Cotta.

Tscheke, J. (2015). Inklusive Didaktik - konstruktivistisch, entwicklungslogisch, themenzentriert-interaktionell. Zeitschrift für Inklusion-online, 10 (4). Verfügbar unter https://www.inklusion-online.net/index.php/inklusion-online/article/ view/319/271 [05.10.2020].

Verband deutscher Musikschulen (Hrsg.). (2017). Spektrum Inklusion - wir sind dabei! Wege zur Entwicklung inklusiver Musikschulen. Grundlagen und Arbeitshilfen. Bonn: Verband deutscher Musikschulen.

Wagner, R. (2002). Bausteine „selbst-verständlichen Musizierens“. In Verband bayrischer Sing- und Musikschulen e.V. (Hrsg.), Musik mit Behinderten an Musikschulen. Grundlagen und Arbeitshilfen, Berichte aus der Praxis, Informationen und Adressen (2. erweiterte Aufl., S. 45-56). Nürnberg: Athmann.

Wagner, R. (2016). Max Einfach - Musik gemeinsam von Anfang an. Regensburg: ConBrio.

Winnerling, S. (2005). Lehrer-Schüler-Verhältnis in pädagogischen Konzeptionen. Anforderungen an eine pädagogische Beziehung aus der Perspektive konstruktivistischer Theoriebildung. Dissertation, FernUniversität Hagen. 

Julia Lutz

\section{Von der Erfahrung über die Reflexion zur Perspektivenübernahme}

\section{Impulse zur Planung und Durchführung musikalischer Aktivitäten in heterogenen Gruppen}

\section{Zur Einführung: Vielfalt als Ausgangspunkt}

Eine Grundschulklasse, 3. Jahrgangsstufe, 25 Kinder. Als Muntermacher nach der Mathematikstunde sprechen die Schüler*innen ein ihnen bekanntes rhythmisches Sprechstück und begleiten es klatschend und stampfend mit Rhythmusbausteinen in verschiedenen Varianten. Unterschiedliche Begleitpatterns erklingen in Folge oder gleichzeitig. Alle Kinder sind dabei. Im Musikunterricht entwickeln sie in Gruppen Ideen zur Gestaltung eines Liedes, das sie vor einiger Zeit kennengelernt haben und begeistert singen. Eine Gruppe arbeitet an einer Bewegungsgestaltung, eine andere stellt die Strophen szenisch dar, eine dritte überlegt sich ein Vor- und Nachspiel und eine Gruppe nutzt die Rhythmusbausteine für eine Begleitung mit Percussion-Instrumenten. Auch bei dieser Aufgabe sind alle dabei und die Freude am gemeinsamen Musikmachen ist erlebbar. Für Beobachtende dagegen kaum sichtbar ist, dass es sich um eine sehr heterogene Lerngruppe handelt, in der Kinder mit unterschiedlichsten Voraussetzungen in Bereichen wie Verhalten, emotionale Entwicklung und Sprache gemeinsam lernen.

Ganz gleich, ob es sich um musikalische Lehr-Lern-Situationen in einer Schulklasse wie der hier beschriebenen handelt, um Unterricht an einer Musikschule oder im Rahmen anderer musikalischer Bildungsangebote: Ein zentrales Moment bei der Planung und Durchführung musikalischer Aktivitäten in einer heterogenen Gruppe ist eine möglichst genaue Kenntnis der Fähigkeiten und Bedürfnisse der beteiligten Personen - auch über ihre musikbezogenen Vorerfahrungen hinaus. Wie drücken sich die Mitwirkenden als Individuen aus und wie bringen sie sich mit ihrem Körper, mit Instrumenten, Sprache, Gestik und Mimik in eine Gruppe ein? Wie interagieren sie mit anderen? Was lässt sich über ihr Konzentrationsvermögen, ihr Sprachverständnis sowie über die geistige und emotionale Entwicklung sagen? Insbesondere im Kontext von Inklusion ist die Frage nach den Voraussetzungen, die jede einzelne Person mitbringt und einbringen kann, ein ganz entscheidender Schritt auf dem Weg zu einer gelingenden musikalischen Interaktion (vgl. Dehler, 2016, S. 23f. und 26f.; Hüttmann, 2018, S. 33; Tischler, 2013, S. 20; Vogel, 2016, S. 6f.). Einen 
Beitrag zur Beantwortung dieser Fragen und damit einen Beitrag, um Unterrichtssituationen entsprechend der individuellen Voraussetzungen der Lernenden zu planen und um deren Verhaltensweisen in konkreten Situationen zu verstehen, kann die Übernahme von Perspektiven der beteiligten Personen leisten (vgl. Dimitrova \& Lüdmann, 2014, S. 3-7).

Dieser Text möchte Impulse geben, bei der Planung und Durchführung musikalischer Lehr-Lern-Situationen immer wieder die Blickrichtung zu ändern und das Unterrichtsgeschehen aus unterschiedlichen Sichtweisen von Beteiligten zu betrachten - besonders, aber nicht nur dann, wenn Inklusion eine Rolle spielt. Dazu werden anhand exemplarischer Beispiele zunächst verschiedene Voraussetzungen von Lernenden thematisiert und Erfahrungs- und Entwicklungsfelder als Impulse zur Reflexion von Lehr-Lern-Situationen vorgestellt (siehe Kapitel 2). Sowohl die individuellen Perspektiven von Lernenden als auch die Erfahrungs- und Entwicklungsfelder regen dazu an, die in Kapitel 3 beschriebenen Praxisbeispiele für musikalische Aktivitäten mit Gruppen in diversen Varianten zu durchdenken, in der Praxis auszuprobieren sowie eigene Erfahrungen zu hinterfragen und zu reflektieren. Dieser Ansatz zur Perspektivenübernahme wurde im Rahmen des Projekts Inklusionsdidaktische Lehrbausteine entwickelt, in das anschließend ein Einblick erfolgt und an das anknüpfend weiterführende Gedanken formuliert werden.

\section{Individuelle Voraussetzungen der Lernenden und Erfahrungsfelder als Reflexionsimpulse}

$J e^{\star}{ }^{\star}$ Lernende hat persönliche Voraussetzungen: bestimmte Fähigkeiten und Fertigkeiten, Kenntnisse, Erfahrungen, Interessen, Verhaltensweisen und individuelle Bedürfnisse. Mit diesen nimmt sie bzw. er am Unterricht teil und bringt sich ein. Eine Lehr-Lern-Situation wiederum hat Einfluss auf die Weiterentwicklung der individuellen Voraussetzungen der Lernenden. Wenn es um das Lernen in inklusiven Gruppen geht, wird häufig nach Defiziten der Lernenden und daran anknüpfend nach Förderschwerpunkten gefragt. Im Folgenden wird der Blick nicht auf bestimmte Förderschwerpunkte gerichtet, sondern auf konkrete Voraussetzungen und damit verbundene individuelle Perspektiven, die Lernende in eine Lehr-Lern-Situation einbringen können. Exemplarisch formulierte und in sechs Kategorien gegliederte individuelle Perspektiven möchten dazu anregen, bei der Planung von Unterricht gezielt Chancen und Herausforderungen zu betrachten, die sich im Zusammenspiel von bestimmten persönlichen Voraussetzungen der Lernenden, Unterrichtsinhalten, Methoden und Medien sowie Sozialformen ergeben. 
Individuelle Voraussetzungen und Perspektiven von Lernenden: Exemplarische Beispiele ${ }^{1}$

Ich bin gerne schnell und vorne dran.

1) Ich möchte am liebsten gleich wissen, wie sich die Sachen anhören - also Instrumente, Gegenstände, eigentlich alles, was es um mich herum so gibt. Darum probiere ich auch immer gleich aus, wie etwas klingt.

2) Manchmal weiß ich nicht, was jetzt passieren soll. Ich weiß nicht, was andere von mir wollen und was ich machen soll.

3) Woher soll ich wissen, wie lange ich in diesem ungewissen Zustand des Wartens verharren soll? Solche Situationen sind mir total unangenehm und fühlen sich wie eine Ewigkeit an.

Es fällt mir schwer, mich zu konzentrieren.

1) Um mich herum sind so viele Geräusche und ich weiß nicht, welche wichtig sind. Gleich die neben mir von meinem Nachbarn oder die anderen, die von draußen hereinkommen?

2) Ich kann immer nur eine Sache auf einmal machen, also entweder zuhören oder zusehen oder spielen oder singen oder schreiben oder ..., aber immer nur eines davon.

3) Welches von all dem um mich herum ist jetzt für mich gedacht - die ganzen Instrumente, die vielen anderen Kinder, die bunten Bilder mit den Notenzeichen hier im Raum?

Ich habe Schwierigkeiten, Sprache zu verstehen.

1) Ich kenne manche Wörter nicht, die meine Lehrperson benutzt.

2) Wenn jemand etwas spricht, erkenne ich nur einzelne Wörter. Die setze ich wie ein Puzzle zusammen, sodass sie für mich einen Sinn ergeben. Irgendetwas wird schon dabei herauskommen.

3) Ich will auf gar keinen Fall, dass jemand merkt, dass ich etwas nicht verstanden habe. Darum sage ich vorsichtshalber mal ,ja“ und bin ansonsten ganz still und mache das nach, was die anderen tun. Damit komme ich irgendwie durch und habe so auch schon einiges gelernt.

1 In jedem Kasten werden mehrere Perspektiven von Lernenden beschrieben. Auf diese Weise soll zum Ausdruck kommen, dass sich die als Überschrift formulierten Voraussetzungen individuell in unterschiedlicher Weise äußern können. 
Ich kann meinen Körper nicht richtig wahrnehmen.

1) Ich bin empfindlich, wenn mir jemand sehr nahekommt.

2) Ich kann mich nur selbst spüren, wenn ich etwas anderes anfasse, also nehme ich Sachen in die Hand. Besonders wohl fühle ich mich, wenn ich andere berühre oder wenn ich berührt werde.

Es tut mir gut, die Aufmerksamkeit anderer zu bekommen.

1) Wenn ich mich ungeschickt anstelle, helfen mir andere. Das finde ich angenehm.

2) Wenn ich nur ein bisschen störe, wird das ignoriert. Das mag ich nicht. Deshalb störe ich mehr und werde lauter. Ich bin da, hört ihr das nicht?

3) Ich kann das! Schau her zu mir!

In einer Gruppe kann ich mich unterschiedlich fühlen und verhalten.

1) Ich fühle mich wohl, wenn die ganze Gruppe aktiv ist.

2) In der Gruppe kann ich mehr leisten als alleine.

3) Ich habe Schwierigkeiten, mich wahrzunehmen, wenn die Gruppe aktiv ist.

4) Es fällt mir schwer, mich vor der Gruppe zu zeigen.

\section{Erfahrungs- und Entwicklungsfelder als Reflexionsimpulse}

Um in musikalischen Lehr-Lern-Prozessen unterschiedliche Voraussetzungen der Lernenden wie die hier exemplarisch beschriebenen zu berücksichtigen, können Erfahrungs- und Entwicklungsfelder wie Selbsterleben und Gruppenerleben, Selbstausdruck und Gruppenausdruck sowie Konzentration und Aufmerksamkeit Orientierung bieten. ${ }^{2}$ Es handelt sich dabei um Aspekte, die im Kontext der persönlichen Entwicklung eines Menschen und der Interaktion mit anderen eine zentrale Rolle spielen. Sie sind auch als grundlegende Momente in der rhythmisch-musikalischen Arbeit präsent (vgl. Peter-Führe, 1997; Hirler, 2003) und finden auch im Bereich der Musiktherapie Berücksichtigung (vgl. z. B. Frohne, 1981). Bezogen auf Unterricht können Erfahrungs-

2 Zum Begriff der Erfahrungsfelder, der im Bereich der musiktherapeutischen und der rhythmisch-musikalischen Arbeit verwendet wird, um das Angebot an Erfahrungen, die einzelne Handlungsfelder und Übungen ermöglichen, zu kategorisieren, vgl. Frohne, 1981, S. 124-129. An die von I. Frohne beschriebenen und von S. Peter-Führe (1997, S. 13-19) aufgenommenen Erfahrungsfelder knüpft der vorliegende Text an, indem ausgewählte Felder als Reflexionsimpulse aufgegriffen, etwas anders akzentuiert, in den Aspekt des Selbst und der Gruppe ausdifferenziert sowie durch das Feld Konzentration/Aufmerksamkeit erweitert werden. Der Begriff der Entwicklungsfelder wird im vorliegenden Reflexionsansatz ergänzt, um die Bedeutung von Erfahrungen für die persönliche Entwicklung zu unterstreichen. 
und Entwicklungsfelder die Funktion einer Brücke zwischen dem Potenzial, das eine bestimmte musikalische Aktivität oder ein Inhalt bietet, und den individuellen Voraussetzungen der Lernenden einnehmen. So ist beispielsweise das Gruppenerleben ein zentrales Feld bei der Einführung und Gestaltung eines mehrstimmigen Sprechstücks. Je nach Voraussetzungen können Lernende dabei auf unterschiedliche Weise unterstützt werden, sich in diesem Feld individuell weiterzuentwickeln.

Im Rahmen musikalischer Handlungsformen bieten Erfahrungs- und Entwicklungsfelder Raum, um sich selbst, die Musik und die Interaktion mit anderen zu erfahren:

Selbsterleben: Musikalische Aktivitäten ermöglichen den Ausführenden, sich selbst zu erleben und ihre Wahrnehmungsfähigkeit zu differenzieren (vgl. Peter-Führe, 1997, S. 13f.): beim Singen und Sprechen oder beim Spiel eines Instruments, indem Klänge, Töne und Worte erzeugt und hörbar werden, oder beim Umsetzen von Musik in Bewegung.

Gruppenerleben: Im Mittelpunkt steht hier die musikalische Interaktion mit anderen. Jedes Individuum erlebt sich in Gruppensituationen nicht nur selbst, sondern auch als Teil der Gruppe, in die es sich sprechend oder singend, musizierend oder sich bewegend einbringt. Die Interaktion auf verschiedenen Ebenen - motorisch, visuell, auditiv, Klänge und Geräusche produzierend bedeutet immer ein Agieren und Reagieren bezogen auf das Handeln der anderen in der Gruppe, und für dieses Zusammenspiel gibt es viele unterschiedliche Wege und Darstellungsformen. Verbunden mit der (Weiter-)Entwicklung der Selbst- und Fremdwahrnehmung kann dies zu einer Erweiterung des Verhaltensrepertoires und zur Stärkung von Toleranz beitragen (vgl. Klöppel \& Vliex, 2004, S. 84).

Selbstausdruck: Sich selbst zu erleben ist eng verbunden mit der Möglichkeit, sich selbst auszudrücken. Dies kann auf verschiedenen Ebenen und in unterschiedlichen Formen geschehen. Zentral sind in musikalischen Situationen die bereits erwähnten Aktivitäten Singen und Sprechen, das Spiel auf Instrumenten und mit Klangerzeugern (z. B. Körper, Alltagsgegenstände) sowie die Verknüpfung von Musik und Bewegung. Emotionen können ebenso wie Gedanken, Stimmungen und Ideen zum Ausdruck gebracht werden, wobei das Verändern von und der spielerische Umgang mit Parametern wie Dynamik, Tempo, Rhythmus und Melodie die Basis für ein variantenreiches und breites Repertoire an Ausdrucksmöglichkeiten darstellt. Sich mit und durch Musik mitzuteilen eröffnet Möglichkeiten, das eigene Ausdruckpotenzial zu entdecken und zu erweitern. Dazu gehören auch Formen, die im Alltag nicht üblich sind und dort keine oder kaum Berücksichtigung finden (vgl. Peter-Führe, 1997, S. 15f.) - etwa die pantomimische Darstellung eines Liedtextes zum Gesang oder die Verklanglichung eines Gedichts mit Instrumenten und Stimme. 
Gruppenausdruck: Sich in der Gruppe auszudrücken bedeutet, in Kontakt zueinander zu treten und gemeinsam etwas darzustellen oder mitzuteilen (vgl. Klöppel \& Vliex, 2004, S. 87). Damit sich jeder ins Gruppengeschehen einbringen, dieses mitgestalten und gemeinsam etwas zum Ausdruck gebracht werden kann, sind Spielregeln bzw. Absprachen notwendig (vgl. Klöppel \& Vliex, 2004, S. 86f.). Deren Bedeutung für das Gruppengeschehen und für den musikalischen Prozess erfahren die Beteiligten besonders dann, wenn die Regeln in Absprache variiert und die daraus resultierenden Unterschiede reflektiert werden.

Konzentration und Aufmerksamkeit: Musik schafft Raum, sich in unterschiedlicher Weise einer Sache zu widmen, seine Aufmerksamkeit ganz auf etwas zu richten - allein ebenso wie in Interaktion mit anderen. Das kann die Konzentration beim Ausführen einer Begleitstimme auf einem Stabspiel ebenso sein wie beim Erkennen des Klangs von Instrumenten mit geschlossenen Augen. Konzentration und Aufmerksamkeit lassen sich sowohl durch eingeführte Rituale wie ein Begrüßungslied zu Beginn der Unterrichtsstunde oder eine Stille-Übung zwischendurch fördern als auch durch Neues, Überraschendes, Unerwartetes - ein Lied mit geschlossenen Augen singen, ein neues, bisher noch nicht verwendetes Instrument ins Repertoire an Klangerzeugern für eine Improvisationsaufgabe aufnehmen.

Als sogenannte Lupen-Aspekte (vgl. Abb. 1, S. 83) werden ausgewählte Erfahrungs- und Entwicklungsfelder im folgenden Kapitel in Verbindung mit konkreten Beispielen aus der Praxis thematisiert. ${ }^{3}$ Ziel ist es, Lehrende beim Nachdenken über Unterricht dazu anzuregen, den geplanten Ablauf bzw. das vergangene Unterrichtsgeschehen hinsichtlich ausgewählter Erfahrungs- und Entwicklungsfelder unter die Lupe zu nehmen. Dieser Blick durch die Lupe kann dazu beitragen, Potenziale ebenso wie mögliche Stolpersteine im Unterrichtsverlauf in Hinsicht auf individuelle Voraussetzungen von Lernenden zu erkennen und in konkreten Unterrichtssituationen zu berücksichtigen.

3 Der Begriff der Lupen-Aspekte wurde im Rahmen eines Kooperationsprojekts zwischen Musik- und Sonderpädagogik an der Ludwig-Maximilians-Universität München geprägt. Mittelpunkt dieser Kooperation war die Entwicklung und Erprobung eines Konzepts für eine Musikdidaktik-Lehrveranstaltung für Lehramtsstudierende. Für nähere Informationen dazu siehe Kapitel 4. Detaillierte Ausführungen zu den individuellen Perspektiven von Lernenden und $\mathrm{zu}$ den Lupen-Aspekten bietet Lutz, 2020 (Kapitel 2). 


\section{Selbsterleben}

\section{Selbstausdruck}

\section{Konzentration /} Aufmerksamkeit

\section{Gruppenerleben}

Abbildung 1: Lupen-Aspekte

\section{Praxisbeispiele unter die Lupe nehmen}

Die Arbeit mit Erfahrungs- und Entwicklungsfeldern als Lupen-Aspekten (Selbsterleben, Gruppenerleben, Selbstausdruck, Gruppenausdruck, Konzentration und Aufmerksamkeit) in Kombination mit ausgewählten individuellen Perspektiven von Lernenden ermöglicht es, bei der Vorbereitung ebenso wie bei der Reflexion von Unterricht persönliche Voraussetzungen der Beteiligten aus verschiedenen Blickwinkeln zu betrachten und Überlegungen zur musikalischen Arbeit im Kontext von Heterogenität auf diese Basis zu stellen. Die beispielhaft beschriebenen Übungen verstehen sich gleichermaßen als Anregung, um Erfahrungen aus der eigenen Praxis zu reflektieren, und als Impuls zum Gedankenspiel bezüglich zukünftiger Unterrichtssituationen.

\subsection{Rhythmusschulung: Rhythmusvariationen mit Körperinstrumenten}

Durch Vor- und Nachmachen werden kurze rhythmische Patterns eingeübt und ausgeführt. Die Grundlage bilden Rhythmusbausteine im Zweivierteltakt: 

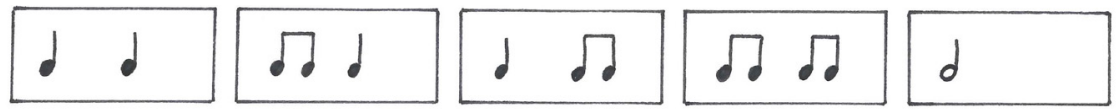

Abbildung 2: Rhythmusbausteine im Zweivierteltakt

Verschiedene Kombinationen dieser Rhythmusbausteine (z. B. Ausführen verschiedener Bausteine nacheinander oder gleichzeitig) in Verbindung mit unterschiedlichen Methoden, Medien und Sozialformen ermöglichen ein variantenreiches Üben. Beispiele:

a) Es werden unterschiedliche Klanggesten verwendet: Klatschen (mit der flachen Hand, mit zwei Fingern, ...), Patschen auf verschiedene Körperteile, Stampfen usw.

b) Die Rolle der vormachenden Person kann die Pädagogin bzw. der Pädagoge ebenso übernehmen wie die Lernenden.

c) Die Bausteine werden mit Wörtern, deren Silbenzahl und Betonung zum Rhythmus passen, unterlegt, z. B. Lö-we, Ka-ka-du, Schild-krö-te, Per-serkat-ze, Bär (Reihenfolge der Wörter entsprechend der Rhythmusbausteine in Abbildung 2).

- Zum Klatschen, Stampfen usw. der Bausteine werden die passenden Wörter gesprochen.

- Ein Wort wird vorgesprochen und die Gruppe führt direkt anschließend das rhythmische Pattern mit Körperklängen aus.

- Eine Person spielt mit einer Klanggeste einen Rhythmusbaustein oder mehrere Rhythmusbausteine in Folge, die anderen nennen die zugeordneten Wörter oder finden neue passende Wörter.

d) Zur Visualisierung können die Bausteine in traditioneller Notenschrift, graphischer Notation (z. B. Balken-Notation, vgl. Abbildung 3) oder in Form von Abbildungen passender Wörter (z. B. Abbildungen der Tiere) angeboten werden. Damit lassen sich auch Folgen aus verschiedenen Bausteinen veranschaulichen oder beim mehrstimmigen Musizieren mehreren Gruppen je ein Baustein zuordnen.

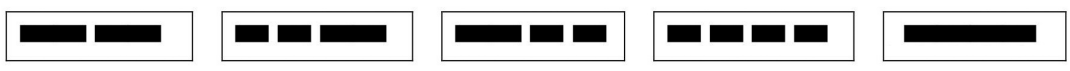

Abbildung 3: Rhythmusbausteine im Zweivierteltakt in graphischer Notation (BalkenNotation)

e) Jeder Baustein wird mit einer vereinbarten Klanggeste (Klatschen, Patschen usw.) ausgeführt.

f) Geübt werden kann in der Gesamtgruppe oder in mehreren kleinen Gruppen. 
g) Jede Kleingruppe legt mit Karten eine Folge aus fünf Rhythmusbausteinen und übt diese. Anschließend präsentiert sie das Ergebnis und die Rhythmusfolgen von mehreren Gruppen werden gleichzeitig musiziert.

h) Ausführen einer Folge aus mehreren Bausteinen als Kanon: Eine Gruppe beginnt, nach dem ersten Baustein setzt die zweite Gruppe von Beginn an ein usw.

i) Für Abwechslung sorgt das Üben in verschiedenen Tempi oder in dynamischen Varianten.

Welche Chancen und welche Herausforderungen bieten diese Übungsformen? Werden sie anhand ausgewählter Erfahrungs- und Entwicklungsfelder als $\mathrm{Lu}$ pen-Aspekte betrachtet, lässt sich feststellen, dass dem Selbsterleben ein breiter Raum eröffnet wird, teilweise verbunden mit der Möglichkeit zum Selbstausdruck: Alle Personen in der Gruppe können mitmachen - mit Körperinstrumenten und Sprache oder mit einer der beiden Musizierformen - und dabei ihren Körper als Medium zum Ausführen von Rhythmen erfahren. Den geklatschten oder gepatschten Rhythmus am Körper zu spüren bietet besonders für Lernende mit eingeschränkter Hörfähigkeit gute Chancen, Musik zu erfahren und zu erleben. In der Rolle des bzw. der Vormachenden haben die Lernenden innerhalb des vorgegebenen Rahmens der Rhythmusbausteine die Gelegenheit, durch eine Auswahl an Bausteinen und Klanggesten, durch ein von ihnen gewähltes Tempo und eine Dynamik, etwas zum Ausdruck zu bringen. Der Aspekt des Gruppenausdrucks und des Gruppenerlebens kommt etwa dann zum Tragen, wenn eine Gruppe selbst eine Folge an Rhythmusbausteinen mit verschiedenen Körperklängen gestaltet, rhythmisch präzise ausführt und bei der Präsentation die Wirkung des exakten Zusammenspiels erfährt. Zur Förderung von Konzentration und Aufmerksamkeit können Übungsformen mit Rhythmusbausteinen in verschiedener Weise einen Beitrag leisten: beim Ausführen kurzer und längerer Folgen von Rhythmusbausteinen nach dem Call-and-Response-Prinzip, beim Umsetzen eines in graphischer Notation dargestellten Rhythmus in eine Folge an Körperklängen oder beim mehrstimmigen Musizieren mit unterschiedlichen Rhythmuspatterns.

Anhand ausgewählter individueller Perspektiven (vgl. S. 79f.) lassen sich Potenziale und Grenzen der Arbeit mit Rhythmusbausteinen mit Fokus auf persönliche Voraussetzungen von Lernenden in den Blick nehmen:

Perspektive: "Ich bin gerne schnell und vorne dran."

Diesen Lernenden kommen die beschriebenen Übungsformen entgegen: Durch die Imitation der rhythmischen Patterns entstehen nur kurze Wartemomente und das exakte Einsetzen kann als motivierende Herausforderung 
betrachtet und geübt werden. Auch der mehrfache Wechsel zwischen verschiedenen Übevarianten kann förderlich sein.

Perspektive: „Es fällt mir schwer, mich zu konzentrieren.“

Für diese Lernenden sind klare Hinweise zur Lenkung der Aufmerksamkeit wichtig, etwa das Mitlesen des auszuführenden Rhythmus anhand entsprechender Rhythmuskärtchen, die visuelle Orientierung an der vormachenden Person oder das Hören auf die gesprochenen Wörter im Rhythmus der entsprechenden Bausteine. Beim mehrstimmigen Zusammenspiel kann es hilfreich sein, die verschiedenen Gruppen an unterschiedlichen Positionen im Raum zu platzieren, so dass die eigene Gruppe einen klaren akustischen und visuellen Anhaltspunkt bietet.

Perspektive: „Ich habe Schwierigkeiten, Sprache zu verstehen.“

Da das aktive Musizieren im Mittelpunkt steht und Lernen durch Imitation dabei eine zentrale Rolle spielt, können diese Lernenden ebenfalls gut in den Übeprozess eingebunden werden. Das Unterlegen der Rhythmusbausteine mit Sprache kann einen Beitrag zur Sprachförderung leisten - insbesondere dann, wenn die Patterns häufig wiederholt werden, die verwendeten Wörter durch Bild- und Wortkarten visuell präsent sind und die Übungen auch mit Begriffen aus anderen Themenbereichen bzw. Wortfeldern durchgeführt werden (außer Tiere z. B. Pflanzen, Jahreszeiten, Essen und Trinken, Kleidung usw.).

Perspektive: „Ich kann meinen Körper nicht richtig wahrnehmen."

Das Ausführen der Rhythmen mit Körperklängen hilft diesen Lernenden, ihren eigenen Körper wahrzunehmen. Zur Förderung einer differenzierten Wahrnehmung können verschiedene Körperinstrumente verwendet werden. Das Erspüren von Rhythmen, die von einer anderen Person auf den eigenen Körper gepatscht oder geklopft werden, kann unter anderem zur Entwicklung von Toleranz gegenüber körperlicher Nähe beitragen.

Perspektive: „Es tut mir gut, die Aufmerksamkeit anderer zu bekommen.“ Wer versehentlich oder bewusst andere Rhythmen spielt als vereinbart, fällt beim gemeinsamen Musizieren kaum auf und findet allein oder mit Hilfe wieder zurück in den gemeinsamen Prozess. In der Rolle der Vormachenden erhalten die Lernenden die Aufmerksamkeit der Gruppe. Voraussetzung, um positive Aufmerksamkeit auf sich zu lenken, ist, dass sie die Rhythmusbausteine sicher ausführen können; ggf. bieten die Vorabauswahl eines oder mehrerer Bausteine und das Mitspielen bzw. Mitsprechen der Lehrperson Unterstützung. 
Perspektive: „In einer Gruppe kann ich mich unterschiedlich fühlen und verhalten."

Je nach gewählter Sozialform können sowohl jene Lernenden, die sich in der Interaktion in einer Gruppe wohl fühlen, als auch jene, die sich in Gruppen schwer selbst wahrnehmen können oder die Schwierigkeiten haben, sich vor der Gruppe zu zeigen, angesprochen werden. Wenn das Musizieren in der Großgruppe problematisch erscheint, kann es mitunter hilfreich sein, in Kleingruppen oder in Partnerarbeit mit den Rhythmusbausteinen zu üben. Das Beobachten anderer in der Rolle der vorzeigenden Person bietet Orientierung, um diese Rolle danach selbst zu übernehmen.

\subsection{Orff-Instrumente: Übungen zum Kennenlernen und zur Wahrnehmungsförderung}

Die folgenden Übungen möchten dazu anregen, sie unter verschiedenen $\mathrm{Lu}$ pen-Aspekten (vgl. S. 80-82) und aus individuellen Perspektiven von Lernenden (vgl. S. 79f.) zu reflektieren. Denkimpulse können folgende Fragen bieten:

- Welche Erfahrungs- und Entwicklungsfelder stehen im Vordergrund und welche Potenziale bieten die Übungen?

- Für welche individuellen Voraussetzungen der Lernenden sind die Übungen förderlich?

- In Verbindung mit welchen individuellen Voraussetzungen der Lernenden sind Herausforderungen festzustellen?

- Wie könnten die Übungen variiert werden, um bestimmten individuellen Voraussetzungen bestmöglich gerecht zu werden?

Erkunden verschiedener Orff-Instrumente (z.B. Handtrommel, Maracas, Klanghölzer, Triangel, Schellenkranz, Becken) mit mehreren Sinnen

- Die unter einem Tuch liegenden Instrumente ertasten (sukzessive, pro Instrument eine andere Person), Form und Material beschreiben; die anderen Personen in der Gruppe vermuten, um welches Instrument es sich handelt; anschließend Instrument zeigen, anspielen und den Klang beschreiben, Instrument benennen und entsprechende Bild-Wort-Karte zuordnen $\rightarrow$ Im Fokus: haptische, auditive und visuelle Wahrnehmung; Sprache

- „Ich höre was, was du nicht hörst, und das klingt so: ...“ - den Klang eines Instruments beschreiben, dann Instrument (verdeckt) anspielen und benennen lassen

$\rightarrow$ Im Fokus: auditive Wahrnehmung 
- „Ich sehe was, was du nicht siehst, und das sieht so aus: ..." - Form, Farbe und Material eines Instruments beschreiben, Instrumente benennen lassen und anschließend zeigen

$\rightarrow$ Im Fokus: visuelle Wahrnehmung; Sprache

- Spielweise eines Instruments pantomimisch darstellen, Instrument benennen lassen und anspielen

$\rightarrow$ Im Fokus: Motorik, visuelle Wahrnehmung

\section{Klänge wandern im Kreis}

Alle sitzen im Kreis, jeder hat ein Rhythmusinstrument zur Verfügung (möglichst viele verschiedene Instrumente verwenden). Reihum spielt jede Person ihr Instrument einmal an. Es werden mehrere Runden durchgeführt und anschließend reflektiert, wobei jede Runde mit einer neuen Aufgabe verbunden ist: Alle spielen ihr Instrument der Reihe nach ...

- ohne weitere Anweisungen;

- erst, wenn das zuvor angespielte Instrument ganz verklungen ist (Herausforderung: Abwarten nach lang klingenden Instrumenten);

- im Grundschlag: Die Pädagogin bzw. der Pädagoge spielt den Grundschlag auf einem gut hörbaren Instrument, der Reihe nach spielt jeweils eine Person mit;

- mit geschlossenen Augen.

\section{Führen und Folgen}

- Die teilnehmenden Personen gehen paarweise zusammen, pro Paar steht ein Instrument zur Verfügung (insgesamt möglichst unterschiedliche Instrumente verwenden).

- Eine der beiden Personen schließt die Augen (evtl. Augenbinde verwenden) und wird von der sehenden mit dem Instrument durch den Raum geleitet.

Von der eigenen Erfahrung in der musikpädagogischen Praxis - bezogen auf die hier beschriebenen oder auf andere Übungen - und deren Reflexion kann der Weg zur Perspektivenübernahme führen. Die Fähigkeit zur Perspektivenübernahme ist zu verstehen „als eine grundlegende Voraussetzung zum Verständnis von Verhaltensweisen, verschiedenen Standpunkten, Gedanken und Emotionen anderer" (Dimitrova \& Lüdmann, 2014, S. 3). Im Hinblick auf die pädagogische Praxis kommt dieser Fähigkeit eine wichtige Bedeutung zu (vgl. Dimitrova \& Lüdmann, 2014, S. 7) - sowohl bei der Planung von Unterricht als auch im Unterrichtsgeschehen, wenn es bestimmte Verhaltensweisen zu erklären und angemessen darauf zu reagieren gilt. Der dargestellte Ansatz zur Perspektivenübernahme anhand von Beispielen individueller Voraussetzun- 
gen der Lernenden und der Lupen-Aspekte wurde im Rahmen eines Kooperationsprojekts zwischen Musik- und Sonderpädagoginnen und Sonderpädagogen entwickelt. Der folgende Einblick in dieses Projekt verdeutlicht den Hintergrund dieses Ansatzes und führt abschließend weiter zu Überlegungen bezüglich Vielfalt als Ausgangspunkt und als Ziel von musikalischer Interaktion in heterogenen Gruppen.

\section{Erfahrungen aus der Lehrer(*innen)bildung und weiterführende Überlegungen}

Um zukünftige Lehrer*innen bereits während des Studiums auf den Unterricht in inklusiven Klassen vorzubereiten, bietet das Projekt Inklusionsdidaktische Lehrbausteine an der Ludwig-Maximilians-Universität München zahlreiche Angebote, an denen verschiedene Fachdidaktiken und unterschiedliche Förderschwerpunkte beteiligt sind. ${ }^{4}$ Im Rahmen des musikpädagogischen Teilprojekts wurde in Kooperation von Musik- und Sonderpädagog $\left({ }^{*}\right.$ inn)en die Basisqualifikation Musik + Inklusion entwickelt. ${ }^{5}$ Es handelt sich um eine Lehrveranstaltung für Studierende des Lehramts Grundschule und Förderschule, die Musik nicht als Studienfach gewählt haben und daher als verpflichtenden Kurs die Basisqualifikation Musik besuchen. ${ }^{6}$ Hier erwerben sie grundlegende Kompetenzen zum Erteilen von Musikunterricht. Die musikalischen Vorkenntnisse und Interessen dieser Studierenden sind sehr unterschiedlich; im neu konzipierten Kurs mit inklusiven Elementen (Basisqualifikation Musik + Inklusion) wird diese Heterogenität bei der Durchführung und Reflexion zahlreicher praktischer Beispiele, die von Liedern und deren Begleitung mit OrffInstrumenten über das Erfinden von Musik mit verschiedenen Klangerzeugern, vielfältige Formen des Hörens von Musik bis hin zur Verbindung von Musik mit Bewegung und Szene reichen, explizit thematisiert. Der Austausch über eigene Erfahrungen führt zum Blick auf unterschiedliche Voraussetzungen von Schüler ${ }^{*}$ inne)n. Über musikbezogene Voraussetzungen hinaus werden auch unterschiedliche Voraussetzungen von Lernenden in anderen Bereichen betrachtet, aus verschiedenen Blickrichtungen reflektiert sowie mögliche

4 Das Projekt startete im Jahr 2015 und wird im Rahmen der Qualitätsoffensive Lehrerbildung des deutschen Bundesministeriums für Bildung und Forschung (BMBF) gefördert.

5 Mitwirkende an der Konzeption der Basisqualifikation Musik + Inklusion: Prof. Dr. Julia Lutz (Musikpädagogik), Dr. Simone Born (Gehörlosen- und Schwerhörigenpädagogik), Ingrid Karlitschek (Pädagogik bei geistiger Behinderung), Mario Riesch (Lernbehindertenpädagogik) sowie Carla Weber (Sprachheilpädagogik und Pädagogik bei Verhaltensstörungen).

6 Pflichtlehrveranstaltung laut LPO I Bayern vom 13.03.2008, § 36 . 
Konsequenzen hinsichtlich der exemplarisch durchgeführten Beispiele diskutiert. Dies geschieht anhand von beispielhaft formulierten Voraussetzungen von Lernenden (vgl. S. 79f.), von denen alle Studierenden jeweils eine individuelle Perspektive schriftlicher Form auf einer Karteikarte erhalten. Im Anschluss an ein Praxisbeispiel, das entweder von den Dozierenden oder von Studierenden angeleitet wird, erfolgt in einer Reflexionsphase der Austausch über eigene Erfahrungen aus der jeweils eingenommenen Perspektive eines bzw. einer Lernenden. Die Praxisbeispiele werden auch anhand der Erfahrungsund Entwicklungsfelder (vgl. S. 80-82) reflektiert.

Am Ende jedes Kurses der Basisqualifikation Musik + Inklusion werden die Studierenden im Rahmen einer projektbegleitenden formativen Evaluation schriftlich anhand eines teilstandardisierten Fragebogens und mündlich im Rahmen eines Abschlussgruppengesprächs zur Lehrveranstaltung auf der Basis eines Leitfadens befragt. ${ }^{7}$ Ziel der Evaluation ist eine kontinuierliche Weiterentwicklung des Kurses auf der Grundlage der Rückmeldungen der Teilnehmenden. Die bisher vorliegenden Evaluationsergebnisse verdeutlichen, dass die Studierenden den Austausch über die eigenen persönlichen Erfahrungen im Rahmen der Praxisbeispiele sowie die gemeinsame Reflexion aus der Perspektive von Lernenden mit äußerst unterschiedlichen Voraussetzungen als sehr gewinnbringend betrachten (Zwischenergebnisse Sommersemester 2016 bis Sommersemester 2018 vgl. Lutz, 2018, S. 30). Die schriftlichen Antworten der Studierenden auf eine offene Frage nach Elementen, die ihnen persönlich besonders relevant erscheinen, sowie Aussagen in den Gesprächen zeigen, dass das eigene Erleben und Erfahrungen im Rahmen des Kurses besondere Bedeutung für die Teilnehmenden haben. Auffallend oft verwenden sie Begriffe wie erfahren und wahrnehmen, Bewusstsein schaffen und sich bewusst werden etwa bezogen auf ihre zukünftige Rolle als Lehrperson und die Gestaltung von Musikunterricht in inklusiven Klassen.

Nicht allein in Bezug auf schulischen Musikunterricht, sondern auch in anderen Bereichen musikpädagogischer Praxis - etwa (Gruppen-)Unterricht an Musikschulen, Chor- und Orchesterproben oder Angebote zur Verbindung von Musik und Tanz für Kinder ebenso wie für Erwachsene - eröffnet der Blick auf das geplante musikalische Geschehen und die sich aktuell vollziehende Interaktion aus der Perspektive der Teilnehmenden Möglichkeiten, die Vielfalt an Potenzial, das sie mitbringen, ebenso zu sehen wie Herausforderungen und Grenzen. Die von diesen Voraussetzungen ausgehende Vielfalt gilt es zu verbinden mit der Vielfalt an Möglichkeiten gemeinsamen Musik-Machens und Musik-Erlebens (vgl. Lutz, 2020, Kapitel 1.1; Anregungen für die musik-

7 Bisher liegen die Evaluationsergebnisse aus sieben Kursen (Sommersemester 2016 bis Sommersemester 2019) vor. Deren Basis sind die ausgewerteten Fragebögen (N $=123$ ) und die Unterlagen aus sieben Gruppenabschlussgesprächen. 
pädagogische Praxis bietet Kapitel 3). Potenziale und Möglichkeiten zu sehen bedeutet dabei nicht, dass in jeder Situation die individuellen Voraussetzungen aller Beteiligten gleichermaßen berücksichtigt werden können. Immer wieder gibt es Situationen, in denen manche Lernende sich nur am Rande einbringen können und von den Lehrenden nicht die volle Aufmerksamkeit erhalten. Entscheidend ist, dies als Pädagogin oder Pädagoge zu wissen bzw. wahrzunehmen und bald darauf eine Situation so zu arrangieren und anzuleiten, dass diese Lernenden die Möglichkeit haben, sich intensiver als zuvor ins Geschehen einzubringen bzw. daran teilzuhaben. Voraussetzung dafür wiederum ist, über ein Repertoire an entsprechenden Handlungsmöglichkeiten - etwa zur inhaltlichen und methodischen Differenzierung - zu verfügen. Zur Entwicklung eines solchen Repertoires kann ein Blick über den jeweils eigenen Bereich hinaus in andere Disziplinen, die eng mit einer inklusiven Musikpraxis verbunden sind, beitragen: Musikpädagogik, Sonderpädagogik und Musiktherapie. Nicht nur gewinnbringend, sondern unverzichtbar erscheint, im Rahmen von Kooperationen sowie von Fort- bzw. Weiterbildungsangeboten Erfahrungen, Eindrücke und Materialien für die pädagogische Praxis mit Expert $\left({ }^{*}\right.$ in$\mathrm{n}$ )en aus anderen Bereichen zu teilen und gemeinsam Wege zu entdecken und auszuprobieren, um die im Kontext von Musik, Heterogenität und Inklusion gegebene Vielfalt zu nutzen und den hier zusammentreffenden Personen vielfältige Begegnungen mit Musik und durch Musik zu ermöglichen.

\section{Literatur}

Dehler, T. (2016). Dabei sein alleine ist noch nicht alles - zum Zusammenspiel von Fachdidaktik und Förderpädagogik im inklusiven Musikunterricht. Diskussion Musikpädagogik, 70, 23-30.

Dimitrova, V. \& Lüdmann, M. (2014). Sozial-emotionale Kompetenzentwicklung. Leitlinien der Entfaltung der emotionalen Welt. Wiesbaden: Springer. https://doi. org/10.1007/978-3-658-04759-7

Frohne, I. (1981). Das Rhythmische Prinzip. Grundlagen, Formen und Realisationsbeispiele in Therapie und Pädagogik. Lilienthal: Eres.

Hirler, S. (2003). Wahrnehmungsförderung durch Rhythmik und Musik (5. Auflage). Freiburg im Breisgau: Herder.

Hüttmann, R. (2018). Ich. Du. Musik. Inklusion. Über die Herausforderungen einer inklusiven Musikpädagogik. Diskussion Musikpädagogik, 79, 31-36.

Klöppel, R. \& Vliex, S. (2004). Helfen durch Rhythmik. Verhaltensauffällige Kinder erkennen, verstehen, richtig behandeln. Kassel: Gustav Bosse Verlag.

Lutz, J. (2018). Gemeinsam Potenziale entdecken. Musik und Inklusion: Ein Einblick in eine interdisziplinäre Kooperation in der Lehrerbildung. neue musikzeitung (nmz), 11, 30. 
Lutz, J. (2020). Musik erleben - Vielfalt gestalten - Inklusion ermöglichen. Anregungen für den Musikunterricht und den Grundschulalltag. Hannover: Kallmeyer in Verbindung mit Klett.

Peter-Führe, S. (1997). Rhythmik für alle Sinne. Ein Weg musisch-ästhetischer Erziehung (4. Auflage). Freiburg im Breisgau: Herder.

Tischler, B. (2013). Musik spielend erleben. Grundlagen und Praxismaterialien für Schule und Therapie. Mainz: Schott.

Vogel, C. (2016). Inklusion und Partizipation. Ansprüche an eine zeitgemäße Musikpädagogik. Diskussion Musikpädagogik, 70, 4-9. 


\section{Ziele und Inhalte des Instrumentalunterrichts mit Menschen mit Behinderung aus der Perspektive österreichischer Musikschullehrkräfte}

Die Perspektive von Musikschullehrkräften auf Ziele und Inhalte des Instrumentalunterrichts mit Menschen mit Behinderung an österreichischen $\mathrm{Mu}-$ sikschulen wird in diesem Beitrag erläutert. Der Artikel basiert auf Inhalten meiner Dissertation (Eibl, 2020) über gesellschaftliche, institutionelle und praktische Aspekte des Instrumentalunterrichts mit Menschen mit Behinderung an österreichischen Musikschulen aus der Perspektive der Lehrkräfte. Nach der Darstellung des methodischen Vorgehens erfolgt die Präsentation der Forschungsergebnisse. Abschließend werden die Ergebnisse zusammengefasst.

\section{Methodisches Vorgehen}

Für das Forschungsprojekt werden insgesamt 22 Lehrkräfte an österreichischen Musikschulen mithilfe von qualitativen Expert( ${ }^{*}$ inn $)$ eninterviews befragt. Dabei wird ein teilstrukturierter Leitfaden verwendet. Die Lehrkräfte unterrichten eine ${ }^{\star} n$ Schüler ${ }^{\star}$ in oder mehrere Schüler*innen mit Behinderung an einem Instrument oder haben bis vor maximal zwei Jahren eine $e^{\star} n$ unterrichtet. Die Befragten haben eine akademische Ausbildung in Instrumental- und Gesangspädagogik absolviert. ${ }^{1}$ Die Schüler ${ }^{\star}$ innen werden im Rahmen des regulären Musikschulunterrichts unterrichtet, also vorrangig im wöchentlichen Einzelunterricht zu je 25 oder 50 Minuten. Privatunterricht, private Musikschulen und inklusive Bandprojekte werden im Rahmen dieses Forschungsprojektes nicht berücksichtigt. Die erhobenen Daten werden mittels der qualitativen Inhaltsanalyse nach Mayring ausgewertet. Dabei wird vorwiegend das allgemeine inhaltsanalytische Ablaufmodell als Orientierung herangezogen (vgl. Mayring, 2015, S. 62; Flick, 2010, S. 411).

1 Eine Ausnahme bilden zwei Lehrer*innen, die ihre künstlerische Ausbildung an einer ausländischen Institution absolviert haben, an der diese Form des Studiums nicht existiert. 
Zur einfacheren Lesbarkeit wird das Sample tabellarisch dargestellt:

\begin{tabular}{|l|l|l|}
\hline Lehrpersonen & \multicolumn{2}{|l|}{} \\
\hline Geschlecht & W & 14 \\
& M & 8 \\
\hline Alter & $30-39$ & 1 \\
& $40-49$ & 9 \\
& $50-59$ & 9 \\
& $>60$ & 3 \\
\hline
\end{tabular}

\begin{tabular}{|l|l|l|}
\hline Schüler*innen & W & 13 \\
\hline Geschlecht & M & 18 \\
& Nicht zugeordnet & 18 \\
\hline Alter & $5-9$ & 4 \\
& $10-19$ & 16 \\
& $20-29$ & 5 \\
& $30-39$ & 1 \\
& $40-49$ & 1 \\
& $>50$ & 2 \\
& Nicht zugeordnet & 20 \\
\hline Instrument & Tasteninstrument (inkl. Akkordeon) & 16 \\
& Blasinstrument & 12 \\
& Schlaginstrument & 8 \\
& Saiteninstrument & 5 \\
& Gesang & 1 \\
& Nicht zugeordnet & 7 \\
\hline Art der & Geistige und Mehrfachbehinderung & 25 \\
Behinderung & Autismus & 4 \\
& Sinnesbehinderung & 3 \\
& Körperliche Behinderung & 2 \\
& Verhaltensbehinderung & 2 \\
& Nicht zugeordnet & 13 \\
\hline
\end{tabular}

Die interviewten 22 Lehrkräfte unterrichten insgesamt 49 Schüler*innen mit verschiedenen Behinderungsarten. Befragt werden acht männliche und vierzehn weibliche Lehrkräfte an österreichischen Musikschulen. Ein zufälliger Schwerpunkt liegt auf der Altersgruppe zwischen 40 und 59 Jahren. Deren Schüler*innen sind vorrangig zwischen zehn und neunzehn Jahren alt. Die Lehrkräfte unterrichten sechzehn Schüler*innen mit Behinderung an einem Tasteninstrument (inkl. Akkordeon), zwölf an einem Blasinstrument. Acht Schüler*innen erlernen ein Schlaginstrument, fünf ein Saiteninstrument und 
eine Schülerin Gesang. Großteils unterrichten die Lehrkräfte Schüler*innen mit einer geistigen oder Mehrfachbehinderung (25 Schüler*innen). Darunter werden auch Schüler*innen mit Down-Syndrom gefasst, obwohl diese Behinderung nach dem ICD 10 (vgl. WHO, 2020) eigentlich als Chromosomenanomalie klassifiziert wird. Die Art der Behinderung der Schüler*innen wird von den Lehrkräften fremdklassifiziert, muss also nicht zwingenderweise korrekt im Sinne des ICD 10 (vgl. WHO, 2020) sein. Sie wird bewusst nicht eingegrenzt, da im Rahmen des Forschungsprojekts auch das Behinderungsverständnis der Lehrkräfte untersucht wird. Informationen über die Behinderungsart entstammen vorrangig der Kommunikation mit den Eltern der Schüler*innen.

\section{Ergebnisse}

Zielsetzungen sowie Unterrichtsinhalte und deren Umsetzung spielen für die Gestaltung der instrumentalpädagogischen Praxis eine wichtige Rolle. Nach einem Überblick über Ergebnisse anderer empirischer Studien zu dieser Thematik wird die Sichtweise der Lehrkräfte darauf präsentiert.

\subsection{Unterrichtsinhalte und deren Umsetzung}

In instrumentalpädagogischer Literatur und empirischen Studien verschwimmen die Begrifflichkeiten Inhalt, Methode und Ziel teils ineinander. Auch in den Interviews mit den Lehrkräften findet keine klare Abgrenzung dieser Begriffe statt. Die hier verwendete Bezeichnung der Unterrichtsinhalte und deren Umsetzung muss deshalb relativ weit gefasst verstanden werden. Unterrichtsinhalte wie Üben, Körper- und Rhythmusschulung, Musiktheorie, Gehörbildung und Instrumentenkunde werden in der instrumentalpädagogischen Literatur auch als Lernfelder bezeichnet (vgl. Busch, 2016, S. 233; Ernst, 1991, S. 41). In empirischen Studien werden breit gefächerte Unterrichtsinhalte und Umsetzungsstrategien beschrieben. Sie reichen von konkreten praktischen Empfehlungen für praktizierende Lehrkräfte bis hin zu bestimmten wünschenswerten Einstellungen. Zum Teil werden auch institutionelle Aspekte, wie z. B. ruhige und störungsfreie Räumlichkeiten (vgl. Hammel, 2004; Probst, 1991) oder genügend Zeitressourcen (vgl. Gerrity, Horton \& Hourigan, 2013) benannt. Konkrete praktische Empfehlungen sind z.B. das Vergrößern und Vereinfachen des Notentextes, Verwenden von Audioaufnahmen und Haltehilfen oder Farben und Symbolen, um Schüler( ${ }^{*}$ inne)n mit Behinderung das 
Erlernen eines Stückes möglich zu machen (vgl. Fitzgerald \& McCord, 2006; Hammel, 2004). Eine klare Struktur soll im Unterricht etabliert werden (vgl. Gerrity et al., 2013; Müller, 2016). Musiker*innen mit einer Hörbehinderung beschreiben das Erlernen von Stücken mithilfe von Vorzeigen und Nachmachen, Fühlen oder Erinnern an ein bestimmtes Körpergefühl als hilfreich (vgl. Fulford, Ginsborg \& Goldbart, 2011). Bei Sehbehinderungen sind Gestik, Sprache, Licht und Fühlen entsprechend zu berücksichtigen (vgl. Baker \& Green, 2016) Schüler( ${ }^{*}$ innen)zentrierung wird als Gelingensbedingung für den erfolgreichen Unterricht betont.

\subsubsection{Unterrichtsinhalte und Umsetzungsstrategien für Schüler*innen mit geistiger oder Mehrfachbehinderung, Autismus oder Verhaltensbehinderung}

Lehrkräfte von Schüler( ${ }^{*}$ inne)n mit geistiger oder Mehrfachbehinderung und Autismus beschreiben insbesondere das Erlernen von Rhythmus und Notenschrift als herausfordernde Inhalte. Deshalb haben sie dafür vielfältige und kreative Umsetzungsstrategien entwickelt. Zusätzlich führen sie als Unterrichtsinhalte gemeinsames Musizieren, Singen, Üben, Komponieren, Nach-Gehör-Spielen und technische Übungen an. Die einzelnen Punkte werden im Anschluss im Detail erläutert.

\section{Rhythmus}

Die befragten Lehrkräfte beschreiben das Erlernen von Rhythmus als Herausforderung im Instrumentalunterricht mit manchen Schüler $\left({ }^{*}\right.$ inne)n mit geistiger und Mehrfachbehinderung oder Autismus. Um deren Rhythmik im Instrumentalspiel zu verbessern, werden von ihnen kreative Strategien entwickelt. So wird fehlerhafter Rhythmus der Schüler*innen durch Begleitung am eigenen oder auf einem Begleitinstrument ausgeglichen. Gleichzeitig wird auf diese Weise zusammen musiziert. Auch das Klatschen des Rhythmus oder die Bewegung im Raum werden als Strategie genannt, um rhythmischen Schwierigkeiten zu begegnen. Die Lehrkraft Teresa beschreibt: ${ }^{2}$ „[Wir haben den] Rhythmus geklatscht, das hat auch Spaß gemacht. Oder dass ich gespielt habe und sie sind im Raum gegangen und haben geklatscht.“

Um das Rhythmusgefühl zu schulen, kann zusätzlich zum Einsatz des Körpers Sprache und Sprachmelodik hinzugezogen werden, zum Beispiel, indem die Stimme bei längeren Noten aufwärtsbewegt wird und dadurch der Noten-

2 Um die Zitate besser lesbar zu machen, werden sie in einer stark geglätteten Form wiedergegeben. 
wert lange genug ausgehalten werden kann. Auch das Spüren des Rhythmus durch die Anwendung von Elementen der Bodypercussion wird als Strategie eingesetzt, zum Beispiel durch Trommeln des Rhythmus am eigenen Körper. Die Reduktion von Komplexität stellt eine weitere Vorgehensweise dar. Vereinfachungen werden zum Beispiel durch sprachliche Simplifizierung vorgenommen: Viertelnoten werden als kurze Noten, Achtelnoten als schnelle Noten definiert und der Rhythmus auf diese Weise zwar nicht akkurat, aber annähernd richtig wiedergegeben.

\section{Notenschrift}

Die Beherrschung der Notenschrift ist manchen befragten Lehrkräften ein grundsätzliches Anliegen in ihrem Instrumentalunterricht. Das Erlernen der Notenschrift stellt sie jedoch teils vor große Herausforderungen im Unterricht mit Schüler( ${ }^{*}$ inne)n mit Behinderung. Trotz intensiver Bemühungen kann sie nicht immer bewältigt werden. Vereinfachen des Notentextes wird als wichtige Umsetzungsstrategie angeführt. Der Notentext wird zum Beispiel einfacher lesbar, wenn er vergrößert, umgeschrieben oder anders arrangiert wird. Die Lehrkraft Monika erzählt: „Und ich habe ihr am Anfang auch die Noten alle größer geschrieben. Also umgeschrieben, weil ich immer das Gefühl gehabt habe, sie sieht es einfach nicht." Auch die Lehrkraft Teresa vereinfacht für einen Schüler mit Behinderung den Notentext. Sie schreibt eigene Arrangements von Klavierstücken, in denen die rechte Hand den Originaltext spielt und die linke Hand nur akkordisch notiert ist. Auf diese Weise sind die Stücke für ihren Schüler spielbar.

Die Verwendung von Hilfssymbolen stellt eine weitere Möglichkeit dar, den Notentext einfacher lesbar zu gestalten. Die Lehrkräfte verwenden dabei die Notennamen, grafische Zeichen, bestimmte Symbole oder Farben. Die Lehrkraft Christine beschreibt, dass im Unterricht mit den Schüler $\left({ }^{*}\right.$ inne)n mit Behinderung Vorzeichen farblich markiert werden. Die Schüler ${ }^{\star}$ innen können zwar Notenlesen, dieses Vorgehen haben sie aber dennoch beibehalten:

„Naja, und da kommt halt dann die glorreiche Idee, dass man das $b$ rot und das fis grün einringelt. Und das haben wir auch beibehalten über die Jahre hinweg. Ich arbeite sonst nie mit Farben. Sie können auch Notenlesen, aber in dem speziellen Fall habe ich das gemacht und es bewährt sich. Also das ist eine neue Methode, die sich aber dann aus der Unterrichtssituation heraus einfach ergeben hat."

Manche Lehrkräfte entwickeln mit ihren Schüler( ${ }^{*}$ inne)n eine eigene, alternative Notenschrift. Die Lehrkraft Franziska beschreibt einen Schüler, dem sie das Notenlesen beibringen wollte. Der Versuch war jedoch nicht erfolgreich. Stattdessen musizieren sie nun im Unterricht nicht nach Noten, sondern nach 
Buchstaben. Außerdem verwenden sie zusätzliche Hilfssymbole, wie zum Beispiel einen Pfeil nach oben, wenn eine hohe Note zu spielen ist. Franziska sagt: „Der ist übrigens einer, der nicht nach Noten spielen kann. Ich habe aber die Noten immer dort liegen und er hat gelernt, er kann nach Buchstaben spielen. Da haben wir uns so eine eigene Notenschrift einfallen lassen, weil das hohe $c$, da mach ich dann einen Pfeil nach oben. Das funktioniert. Ich habe lange versucht, ihm wirklich Noten beizubringen, diese Abstraktion ist nicht gelungen. So schreib ich halt dann immer über die Noten die Notennamen drüber und mit Pfeilen und so weiter."

\section{Zusammenspiel}

Das gemeinsame Musizieren mit der Lehrkraft während der Unterrichtsstunde stellt einen weiteren Unterrichtsinhalt dar. Dabei ist zu unterscheiden, ob die Lehrkraft auf dem gleichen, unterrichteten Instrument oder auf einem Begleitinstrument musiziert. Auch vierhändiges Spiel wird beschrieben, wenn es das Instrument ermöglicht. Gemeinsames Musizieren wird auch in Form von gemeinsamem Singen und Tanzen praktiziert. Die Schülerinnen spielen am Instrument, während die Lehrperson dazu singt oder tanzt, oder umgekehrt. Ebenso wird zu Audioaufnahmen musiziert, zum Beispiel zu mitgebrachten CDs oder über diverse Internetkanäle. Das erfreut sich laut den Lehrkräften großer Beliebtheit, da die Schüler*innen auf diese Weise zu ihren Lieblingsliedern bzw. mit ihren Lieblingsmusiker( ${ }^{\star}$ inne)n musizieren können.

\section{Singen}

Singen stellt nicht nur eine Form des gemeinsamen Musizierens dar, sondern wird auch als eigener Unterrichtsinhalt angeführt. Für manche Lehrkräfte ist Singen im Unterricht ein wichtiger Bestandteil. Die Lehrkraft Angelika erzählt, dass sie in ihrem Instrumentalunterricht mehr singt, seit sie Schülerinnen mit Behinderung unterrichtet. Bevor sie neue Lieder am Instrument erarbeiten, singt sie diese mit ihnen. Falsche Töne spielen dabei keine Rolle. Das Singen bereitet auch anderen Schüler $\left({ }^{*}\right.$ inne)n große Freude, weshalb sie dies auch in den Instrumentalunterricht mit nichtbehinderten Schüler ${ }^{*}{ }^{*}$ inne)n aufgenommen hat. Angelika erzählt:

„Ich mach vielleicht mehr mit Singen als ich vorher gemacht hab. Indem ich die Lieder singen lasse, bevor ich sie spiele. Oder dazu singe. Also viel mehr mit Singen mache. Auch wenn die Kinder nicht sauber singen, das ist völlig wurscht." 
Üben

Häufiges Wiederholen und Üben im Instrumentalunterricht wird von manchen Lehrkräften als Unterrichtsinhalt genannt. Dabei beschreiben sie unterschiedliche Wahrnehmungen hinsichtlich dessen, ob das den Schüler $\left({ }^{*}\right.$ inne) $n$ Freude bereitet. Auf der einen Seite wird häufiges Wiederholen von Schüler ${ }^{*}$ inne)n verweigert, da es als langweilig empfunden wird, auf der anderen Seite nehmen die Schüler*innen es mit Freude an. Die Lehrkraft Gertrude beschreibt ersteres: „Wenn ich sie manchmal überreden kann, probieren wir es halt ein zweites Mal. Aber das Wiederholen, das ist eher fad.“

Wiederholen im Sinne eines gemeinsamen Übens ist auch der Lehrkraft Monika wichtig. Sie übt mit ihrem Schüler viel während der Stunde, auch um ihm zu zeigen, wie richtiges Üben funktioniert. Monika beschreibt das als sehr anstrengend und fordernd für ihren Schüler.

Im Gegensatz dazu geht die Lehrkraft Angelika davon aus, dass oftmaliges Wiederholen von Stücken für die Schüler*innen freudvoll ist. Sie geht davon aus, dass den Schüler ${ }^{*}$ inne)n nicht immer etwas Neues präsentiert werden muss und häufige Wiederholung das bereits vorhandene Wissen festigt und ermöglicht, bereits bekannte Lieder noch besser zu beherrschen.

Die Lehrkräfte führen zusätzlich das Erlernen von Musikstücken nach Gehör, Technikübungen am Instrument und Komponieren mit den Schüler $\left({ }^{*}\right.$ inne)n mit geistiger oder Mehrfachbehinderung, Autismus und Verhaltensbehinderung als Unterrichtsinhalte an. Die Verwendung eines Hausaufgabenheftes, ritualisierte Abläufe und Schüler(*innen)zentrierung stellen weitere inhaltliche Bestandteile ihres Unterrichts dar.

\subsubsection{Unterrichtsinhalte und Umsetzungsstrategien für Schüler ${ }^{\star}$ innen mit Sinnesbehinderung und körperlicher Behinderung}

Von interviewten Lehrkräften blinder Schülerinnen werden keine gesonderten Unterrichtsinhalte beschrieben. Das Erlernen von neuen Stücken scheint vordergründiger Unterrichtsinhalt zu sein. Allerdings werden gesonderte Umsetzungsstrategien zum Erlernen neuer Stücke erwähnt. Tasten, Fühlen und Hören sind dabei die zentralen Parameter. Neue Stücke werden vorrangig über das Gehör gelernt, weshalb die Verwendung von Audioaufnahmen im Instrumentalunterricht und beim Üben eine bedeutende Position einnimmt.

Für Schüler*innen mit Cochlea-Implantat werden ebenso keine bestimmten Unterrichtsinhalte genannt. Auch werden keine speziellen Vorgehensweisen im Unterricht beschrieben. Der Unterricht unterscheidet sich nicht von 
jenem mit nichtbehinderten Schüler( ${ }^{*}$ inne)n. Eine Lehrkraft beschreibt lediglich, dass sie ein besonderes Augenmerk auf ihre eigene, deutliche Aussprache legt. Dies sei aber eigentlich gar nicht notwendig.

Lehrpersonen von Schüler( ${ }^{*}$ inne)n mit einer körperlichen Behinderung beschreiben Unterrichtsinhalte und deren Umsetzung entsprechend denen von nichtbehinderten Schüler $\left({ }^{*}\right.$ inne)n. Der Instrumentalunterricht wird als normal wahrgenommen, wobei nicht näher definiert wird, was darunter verstanden wird. Möglicherweise ist damit der instrumentale Leistungsfortschritt gemeint, der jenem von nichtbehinderten Schüler $\left({ }^{*}\right.$ inne)n gleicht. Unter Umständen hängt diese Wahrnehmung auch damit zusammen, dass die Behinderung der Schüler*innen kaum ist und im Musikschulalltag keine Einschränkungen für Lehrer*innen oder Schüler*innen verursacht.

\subsection{Zielsetzungen}

In der instrumentalpädagogischen Literatur werden Ziele unterschieden nach ihrer Ausdifferenzierung, zum Beispiel Leit-, Grob- oder Feinziele, oder auch nach verschiedenen Dimensionen, zum Beispiel motorische, emotionale oder kognitive Ziele (vgl. Ernst, 1991; Losert, 2015). Die Zielsetzungen der befragten Lehrkräfte unterscheiden sich jedoch vorrangig darin, ob sie musik- und instrumentenbezogene oder außermusikalische Ziele sind, weshalb sie dementsprechend gegliedert werden.

\section{Musik- und instrumentenbezogene Ziele}

Bei musik- und instrumentenbezogenen Zielsetzungen lassen sich aus den Erzählungen der Lehrkräfte fünf verschiedene Schwerpunkte erkennen. Das sind die Freude am Musizieren, die Freude am Musizieren in Verbindung mit einer Steigerung des Könnens am Instrument, die Ausdifferenzierung des instrumentalen Könnens, die individuelle Förderung der Schülerin oder des Schülers und die Auftrittsorientierung. ${ }^{3}$ Diese Schwerpunkte in den Zielsetzungen der befragten Lehrpersonen gehen teils fließend ineinander über und sind nicht immer klar voneinander abgrenzbar.

\section{Freude am Musizieren}

Freude am Musizieren ist manchen Lehrkräften im Instrumentalunterricht mit den Schüler ( ${ }^{*}$ inne)n mit Behinderung ein wichtiges Anliegen. Diese Zielsetzung kann auch in Verbindung mit einem verminderten Leistungsanspruch

3 Die Auftrittsorientierung als Zielsetzung ist darauf ausgerichtet, Schüler*innen auf das nächste Konzert oder anstehende Prüfungen vorzubereiten. 
an Schülerinnen mit Behinderung stehen. Die Lehrkraft Xaver sagt: „Und beim [Schülername], da habe ich jetzt nicht diese Erwartungsansprüche und da muss jetzt unbedingt was weitergehen. Da reichts mir schon, wenn er gern kommt."

Teils müssen anfängliche Erwartungen im Laufe des Unterrichts zurückgeschraubt und eine weniger hohe Leistungsorientierung akzeptiert werden. Die Lehrkraft Otto beschreibt diese Veränderung seiner anfangs leistungsorientierten Ziele als eine Art der Weiterentwicklung als Lehrkraft, in der alte Zielsetzungen und Erwartungen verworfen wurden. Das Resultat daraus ist das Ziel der Freude am Musizieren:

„Meine Ziele waren am Anfang sehr hoch und so nach fünf, sechs oder sieben Stunden hat es mir dann so den Schalter umgelegt und denk ich mir: ,Naja, das geht ja gar nicht so wie ich glaub.' Also das mit den Noten, ich hab ihm weißt eh, Noten aufgeschrieben und einfach probiert und Viertelnoten und irgendeinen Blödsinn ((lacht)) - vergiss es. Kannst alles wegräumen. Also jetzt im Nachhinein denk ich mir ja klar, das gehört einfach weggeräumt - das braucht man nicht. Das Ziel ist einfach, dass man miteinander musiziert. Dass man zum Teil einfach auch frei wirklich frei improvisiert. Also, das was ihm ja völlig Spaß macht. Also das Ziel ist einfach miteinander musizieren. Punkt."

Nicht immer ist das Zurückschrauben von bestimmten Zielsetzungen für die Lehrkraft ein positives Erlebnis. Es kann auch mit Resignation einhergehen. Das beschreibt Gertrude, die sich mit dem Heruntersetzen bestimmter Ansprüche im Unterricht mit der Schülerin mit Behinderung eher schwergetan hat. Sie beschreibt dieses Zurücksteigen von ursprünglichen Zielsetzungen als eine Art Abstieg. Sie sagt:

„Dann bin ich halt von meinem Anspruch, den man hat als Musikschullehrer, einfach sukzessive immer weiter runtergestiegen. Weil ich gedacht hab, aha das geht nicht. Da steigen wir noch ein Stückerl runter. Man muss sich natürlich da irgendwie innerlich arrangieren damit, gell? Im Lauf der Jahre bin ich halt einfach immer wieder, immer noch gelassener worden und völlig vom gewohnten Anspruch weggekommen."

\section{Freude am Musizieren und Steigerung des Könnens}

Freude am Musizieren kann auch in Verbindung mit einem bestimmten Leistungsanspruch an die Schüler*innen einhergehen. Der Spaß am Musizieren steht zwar noch im Vordergrund, gleichzeitig führen die befragten Lehrkräfte aber auch eine bestimmte Erwartungshaltung an die Schüler*innen an. Ein nicht genauer benannter Lerneffekt soll erzielt werden. Der Leistungsanspruch gestaltet sich dabei unterschiedlich hoch. Die Lehrkraft Doris wünscht 
sich, dass die Schüler*innen neben der Freude auch etwas mitnehmen und der Unterricht musikalische Inhalte aufweist. Sie sagt:

„Eigentlich nur, dass es den Menschen Freude macht. Aber schon, dass sie auch was mitnehmen. Also nicht, dass wir dann nur irgendwie was tun, was gar nichts mit Musik zu tun hat oder nur Spielen oder so. Also es soll schon Musik dabei sein.“

Höhere Erwartungen hat dagegen die Lehrkraft Angelika, die neben Freude am Unterricht bereits von einer Ausdifferenzierung des musikalisch-instrumentalen Könnens spricht. Angelika beschreibt:

„Es geht in erster Linie um die Freude des Musik-Empfindens. Also Musik machen und dabei Freude zu haben. Und dann immer differenzierter immer fortgeschrittener."

Der Übergang zur Zielsetzung der Ausdifferenzierung des instrumentalen Könnens ist fließend. Die Freude am Musizieren tritt für Lehrkräfte, die darauf ihren Schwerpunkt legen, jedoch ein wenig mehr in den Hintergrund. Steht die Ausdifferenzierung des instrumentalen Könnens im Vordergrund, wollen die Lehrkräfte beständig das instrumentale Können sowie musikalische Kenntnisse und Fähigkeiten der Schüler*innen verbessern. Die Lehrkraft Monika beschreibt zum Beispiel das Ziel der Verbesserung der rhythmischen Fähigkeiten der Schülerin mit Behinderung:

„Bei ihr habe ich halt das Ziel, dass sie rhythmisch einfach besser wird, das selber in den Griff kriegt. Weil von den Noten und so weiter das kann sie. Das ist überhaupt kein Problem. Aber dass sie sich selber einen Rhythmus erarbeiten kann."

\section{Individuelle Förderung}

Da individuell verschieden ist, welche Bereiche bei welcher Schülerin und welchem Schüler verbesserungswürdig erscheinen, geht diese Zielsetzung fließend über in die der individuellen Förderung von Schüler $\left({ }^{*}\right.$ inne)n. Schüler*innen sollen je nach Persönlichkeit und Leistungsvermögen bestmöglich gefördert werden. Das Bemühen um eine ganzheitliche Sichtweise auf den Instrumentalunterricht zeigt sich. Aus dieser Zielsetzung ergeben sich teils große Spannungen zu den standardisierten Anforderungen des Lehrplans, die von den Lehrkräften umgesetzt werden sollen. Die bestmögliche individuelle Förderung lässt die Erfüllung dieser Anforderungen laut den befragten Lehrkräften nicht immer zu. Der Lehrkraft Hans ist wichtig, dass eine Entwicklung der Schülerin oder des Schülers erkennbar ist. Individuell vereinbarte Ziele zwischen Eltern, Schüler*in und Lehrerperson haben dabei wenig mit Lehrplanzielen zu tun. Hans erzählt: 
„Die Ziele sind insofern da, dass ich sag es muss unterm Strich nachher schon auch merkbar sein, dass man was musikalisch mit dem Kind gemacht hat. Und es muss auch klipp und klar definiert sein, dass ich wirklich sagen kann: ,Ok eine gewisse Entwicklung ist da.' Jetzt nicht mit einem Lernziel in ein, zwei, drei Jahren laut KOMU-Lehrplan, dem wir unterstellt sind, sondern wirklich den individuellen Zielen die wir, hier in dem Fall Eltern und meine Wenigkeit und Schüler vereinbaren."

\section{Außermusikalische Ziele}

Als außermusikalische Ziele werden jene Zielsetzungen bezeichnet, die in die Lebenswelt der Schüler*innen außerhalb des Instrumentalunterrichts eingreifen und nicht in direkter Verbindung mit dem Erlernen des Instruments stehen. Sie werden hier auch als Transfereffekte bezeichnet. Die Lehrkräfte beschreiben Ziele wie vermehrten gesellschaftlichen Anschluss der Schüler*innen, die Förderung ihrer Selbstständigkeit oder eine Verbesserung von behinderungsbedingten Schwierigkeiten.

Außermusikalische Zielsetzungen wie vermehrte Autonomie oder gesellschaftlicher Anschluss werden dabei in der Verantwortung der Lehrkraft gesehen, obwohl sie zeitlich und räumlich außerhalb des Instrumentalunterrichts liegen. Die Förderung der Selbstständigkeit von Schüler $\left({ }^{*}\right.$ inne $) n$ mit Behinderung wird zum Beispiel durch das Ziel des selbstständigen Zurücklegens des Wegs zur Musikschule realisiert. Der Instrumentalunterricht wird außerdem als Mittel zur verbesserten sozialen Integration der Schülerinnen mit Behinderung gesehen. Das Erlernen des Musikinstruments rückt in den Hintergrund und wird Mittel zum Zweck. Die Lehrkraft Jan beschreibt das so:

„Sie sind wirklich mehr sozial integriert über das Instrument, über die $\mathrm{Mu}$ sikschule. Also das ist schon eine super Sache. Das [Instrument] ist nur ein Mittel. Nur so wie irgendein Katalysator."

Der Ausgleich von behinderungsbedingten Schwierigkeiten kann sich laut der Lehrkraft Jan in der Zielsetzung einer verbesserten Koordination oder Körperbeherrschung ausdrücken. Auch empirische Studien, die im Zusammenhang mit Musikpädagogik und Menschen mit Behinderung stehen, verweisen häufig auf diese Transfereffekte (vgl. Colwell, 2002; Darrow, 2011; de l'Etoile, 2005; Levy, Jindal-Snape \& Robb, 2017; Müller, 2016; Probst, 1991). Diese dienen zum Teil auch als Rechtfertigung und Legitimation für den Unterricht von Menschen mit Behinderung.

Andere Lehrkräfte reagieren auf außermusikalische Zielsetzungen mit scharfer Kritik. Sie werden als Überforderung des eigenen Aufgaben- und Verantwortlichkeitsbereiches gesehen. Zudem wird Musik nicht als Mittel zu einem bestimmten Zweck verstanden, sondern als etwas Absolutes. Die Lehrkraft Angelika beschreibt das wie folgt: 
„Ich finde Musik ist etwas Absolutes und ist nicht Mittel zum Zweck. Ich bin nicht dafür da, die Behinderungen zu erleichtern oder zu verändern. Das kann ich nicht und das ist nicht mein Job. Wir sind Musiklehrer und keine Lebensberater. Ich glaube, das ist wichtig, dass man sich da selber zurücknimmt. Also so sehr man sich bemüht und sicher dem Menschen auch hilft, aber ich helfe demjenigen genauso wie den anderen Kindern, Zugang zur Musik zu finden und dadurch vielleicht irgendwas Schönes zu haben. Nicht mehr und nicht weniger."

\section{Zusammenfassung}

In den Zielsetzungen und Unterrichtsinhalten sowie deren Umsetzung ist eine hohe Schüler( ${ }^{*}$ innen)zentrierung erkennbar. Vielfältige und kreative Wege werden beschrieben, wie Lehrkräfte in ihrer Praxisgestaltung auf die individuellen Voraussetzungen der Schülerinnen mit Behinderung Bezug nehmen. Die Art der Behinderung scheint sowohl in den Zielsetzungen wie auch für Unterrichtsinhalte und deren Umsetzung eine wichtige Rolle einzunehmen.

Bezüglich der Unterrichtsinhalte und deren Umsetzung beschreiben die befragten Lehrkräfte das Erlernen von Rhythmus und Notenschrift als besondere Herausforderung für manche Schülerinnen mit einer geistigen oder Mehrfachbehinderung und Autismus. Dieser begegnen sie durch an die Möglichkeiten der Schüler*innen angepasste Vorgehensweisen. Außerdem werden diverse weitere Unterrichtsinhalte wie gemeinsames Musizieren, Singen und Üben angeführt. Die Unterrichtsinhalte werden sehr abwechslungsreich dargestellt. Bei Schüler(*inne)n mit Sinnesbehinderung und körperlicher Behinderung scheint das Erlernen neuer Stücke vorrangig zu sein. Zwar werden Strategien für blinde Schüler*innen genannt, die Tasten, Fühlen und Hören als Zentrum haben, es werden aber keine gesonderten Unterrichtsinhalte erwähnt.

Die Zielsetzungen der Lehrkräfte unterteilen sich in musik- und instrumentenbezogene und außermusikalische Ziele. Musikbezogene Ziele enthalten die Schwerpunkte Auftrittsorientierung, Freude am Unterricht, die Ausdifferenzierung des instrumentalen Könnens und individuelle Förderung. Die Zielsetzung Freude am Musizieren geht zum Teil mit einem verminderten Leistungsanspruch an die Schüler*innen einher. Außermusikalische Zielsetzungen (vermehrte Selbstständigkeit der Schüler*innen, gesellschaftlicher Anschluss sowie Ausgleich der Behinderung) werden im Besonderen für behinderte Schüler*innen genannt. Sie erfahren von anderen befragten Lehrkräften scharfe Kritik. Sie werden als außerhalb des Verantwortungsbereiches von Musikschullehrkräften verortet. Zudem wird kritisiert, dass Musik bzw. der Instrumentalunterricht dann nur mehr Mittel zum (außermusikalischen) Zweck sei. 


\section{Literatur}

Baker, D. \& Green, L. (2016). Perceptions of schooling, pedagogy and notation in the lives of visually-impaired musicians. Research Studies in Music Education, 38 (2), 193-219. https://doi.org/10.1177/1321103X16656990

Busch, B. (Hrsg.). (2016). Grundwissen Instrumentalpädagogik. Ein Wegweiser für Studium und Beruf. Wiesbaden: Breitkopf \& Härtel.

Colwell, C. (2002). Learning Disabilities in the Music Classroom: Implications for the Music Educator. Applications of Research in Music Education, 21 (2), 9-16. https://doi.org/10.1177/87551233020210020401

Darrow, A. (2011). Adaptive Instruments for Students with Physical Disablities. General Music Today, 25 (2), 44-46. https://doi.org/10.1177/1048371311423287

De I'Etoile, S. (2005). Teaching Music to Special Learners: Children with Disruptive Behavior Disorders. Music Educators Journal, 91 (5), 37-43. https://doi.org/ $10.2307 / 3400141$

Eibl, J. (2020). Instrumentalunterricht mit Menschen mit Behinderung. Gesellschaftliche, institutionelle und praktische Aspekte aus der Perspektive der Lehrkräfte an österreichischen Musikschulen. Unveröffentlichte Dissertation. Universität für Musik und darstellende Kunst Wien.

Ernst, A. (1991). Lehren und Lernen im Instrumentalunterricht. Ein pädagogisches Handbuch für die Praxis. Mainz: Schott.

Fitzgerald, M. \& McCord, K. (2006). Children with Disabilities Playing Musical Instruments. Music Educators Journal, 92 (4), 47-52. https://doi.org/10.2307/ 3401112

Flick, U. (2010). Qualitative Sozialforschung. Eine Einführung. Hamburg: Rowohlt.

Fulford, R., Ginsborg, J. \& Goldbart, J. (2011). Learning not to listen: the experiences of musicians with hearing impairments. Music Education Research, 13 (4), 447-464. https://doi.org/10.1080/14613808.2011.632086

Gerrity, K., Horton, P. \& Hourigan, R. (2013). Conditions That Facilitate Music Learning Among Students with Special Needs: A Mixed-Methods Inquiry. Journal of Research in Music Education, 61 (2), 144-159. https://doi.org/ $10.1177 / 0022429413485428$

Hammel, A. (2004). Inclusion Strategies That Work. Music Educators Journal, 90 (5), 33-37. https://doi.org/10.2307/3400021

Levy, S., Jindal-Snape, D. \& Robb, A. J. (2017). Disability, personalization and community arts: exploring the spatial dynamics of children with disabilities participating in inclusive music classes. Disability \& Society, 32 (2), 254-268. https:// doi.org/10.1080/09687599.2016.1276433

Losert, M. (2015). Die Kunst zu unterrichten. Grundlagen der Instrumental- und Gesangspädagogik. Mainz: Schott.

Mayring, P. (2015). Qualitative Inhaltsanalyse. Grundlagen und Techniken. Weinheim, Basel: Beltz. 
Müller, S. (2016). Musizieren mit Menschen mit Behinderung. In B. Busch (Hrsg.), Grundwissen Instrumentalpädagogik. Ein Wegweiser für Studium und Beruf (S. 178-186). Wiesbaden: Breitkopf \& Härtel.

Probst, W. (1991). Instrumentalspiel mit Behinderten. Ein Modellversuch und seine Folgen. Mainz: Schotts Söhne.

\section{Online-Quellen}

WHO (2020). Internationale statistische Klassifikation der Krankheiten und verwandter Gesundheitsprobleme (10. Revision), German Modification. Verfügbar unter: https://www.dimdi.de/static/de/klassifikationen/icd/icd-10-gm/kode-suche/ htmlgm2020/ [27.07.2020]. 


\section{Die Bedeutung des Orff-Schulwerks für inklusiven Musikunterricht}

Der Ansatz des Orff-Schulwerks kann Modelle für differenzierten Unterricht anbieten und inklusives Lehren in vielfältiger Weise unterstützen. Am Beispiel des Uhu-Lieds von Gerda Bächli wird gezeigt, wie dieser Ansatz, bzw. der der Elementaren Musik- und Tanzpädagogik, es ermöglicht, ein Thema durch viele Aktivitäten und auf verschiedenen Ebenen zu entwickeln und dabei die für inklusives Lehren wesentlichen Aspekte innere Differenzierung und Zusammenarbeit an einem gemeinsamen Thema zu berücksichtigen.

Acht Kinder zwischen vier und zehn Jahren - manche haben eine Behinderung bzw. eine Entwicklungsstörung - bilden eine Personenschlange, einen imaginären Zug, der mit der Lehrerin als Lokomotive in den Unterrichtsraum im Orff-Institut der Universität Mozarteum Salzburg zur wöchentlichen Musik- und Bewegungsstunde einfährt. Jedes Kind ist ein Individuum mit seinem persönlichen Hintergrund, seinen Erfahrungen, Interessen, Bedürfnissen und Fähigkeiten. Die Zusammensetzung der Gruppe ändert sich von Jahr zu Jahr, manche Kinder bleiben nur ein Jahr während andere über mehrere Jahre hinweg teilnehmen.

Die Herausforderung beim Unterrichten dieser und sonstiger Gruppen liegt stets darin, verschiedene Interessen, Erfahrungen, Fähigkeiten und Begabungen zu unterstützen, ferner geeignete Inhalte und Methoden so zu wählen, dass jedes Kind nicht nur auf seinem eigenen Niveau teilnehmen, die Aufgaben verstehen und somit lernen kann, sondern auch emotional beteiligt ist, Fantasie und Kreativität einsetzen, die Aktivitäten genießen und das Selbstvertrauen stärken kann. Die Stunden und die einzelnen Tätigkeiten müssen den Bedürfnissen, Interessen und dem Entwicklungsstand aller Kinder entsprechen.

\section{Das Konzept des Orff-Schulwerks}

Elementare Musik- und Bewegungserziehung wurde von Carl Orff und Gunild Keetman begründet und wird auch als Orff-Schulwerk bezeichnet. ${ }^{1}$ Das Wort Schulwerk bezeichnet ein Lehrwerk und war ursprünglich für die Arbeit mit Kindern in Grundschulen gedacht.

1 Nicht zu verwechseln mit den gedruckten Bänden Orff-Schulwerk/Musik für Kinder, die in den Jahren 1950-1954 als Modelle für Lehrende veröffentlicht wurden. 
Seine Verwendbarkeit darüber hinaus für Bereiche wie Vor-, Sonderschule und inklusive Pädagogik, Therapie, Community-Arbeit und Senior( ${ }^{*}$ inn)en wurde schon früh erkannt (wie z.B. von Wilhelm Keller, 1971; Karl Hofmarksrichter, 1962; Hans Wolfgart, 1971, 1975.) Seit den 1960er Jahren wurden die Ansätze des Schulwerks in den genannten Bereichen von Lehrerkolleg( ${ }^{*}$ inn)en entwickelt und dokumentiert sowie die fundamentalen Ideen aus dem Schulwerk an ihre speziellen Zielgruppen angepasst. Diese Entwicklungen waren und sind auch heute noch wegen ihrer humanistischen Orientierung eine logische Fortsetzung, weil der Gedanke, mit allen Alters- und Begabungsgruppen zu arbeiten, dem Orff-Schulwerk inhärent ist. ${ }^{2}$ Wilhelm Keller, Pionier der inklusiven Musik- und Bewegungspädagogik im deutschsprachigen Raum, erkannte, dass für Orff die Entwicklung der ganzen Persönlichkeit zentral war und nannte diese Zielsetzung musica humana (vgl. Salmon, 2012).

Im Kontext des Orff-Schulwerkes steht jedes Individuum, sei es ein Baby, ein kleines Kind, ein Jugendlicher oder Erwachsener mit seiner jeweils eigenen Entwicklung, seinem Hintergrund, seiner Kultur und seinen Interessen, seinen Fähigkeiten und Bedürfnissen im Zentrum der Aufmerksamkeit. Die humanistische Orientierung erkennt das kreative Potenzial, das jedem menschlichen Wesen innewohnt, an und betrachtet es als eine der Aufgaben der Lehrperson, diese Anlagen zu fördern.

Das Orff-Schulwerk ist ein Konzept aktiver und kreativer Musikpraxis für alle - „die Verwirklichung einer ursprünglichen musikalischen Potenz, die in jedem Individuum angelegt ist" (Keller, 1984, S. 801). Es besteht unabhängig von einem bestimmten Alter, besonderen Talenten oder Behinderungen. Es beschreibt vielmehr das musikalische Interagieren zwischen Personen mit ihren jeweiligen individuellen Fähigkeiten. Da jede Spielerin, jeder Spieler eines Ensembles eine individuell angepasste Rolle oder Aufgabe erhält, kann sie oder er als vollwertiges Gruppenmitglied teilnehmen. Es erlaubt Menschen aller Begabungen, in einer Gruppe zusammen zu spielen, ohne dass ein Mitglied über- oder unterfordert wäre. Aufgaben und Rollen werden an die Fähigkeiten der Individuen angepasst, statt dass sich die Gruppe an eine fixierte Form anpassen muss (vgl. Keller, 1996).

„Es gilt, die Fantasie und Erlebniskraft zu wecken in einer Frühzeit, die dafür einzigartig prädestiniert ist. Alles, was das Kind in dieser Frühzeit erlebt, was in ihm geweckt und gepflegt wird, ist maßgeblich für das ganze Leben. Nie wieder Einholbares kann in diesen Jahren verschüttet werden." (Orff, 1964, zitiert nach Haselbach, 2011, S. 157)

2 Siehe dazu Orff-Schulwerk Heute 99, 2018. 


\section{Prinzipien des Orff-Schulwerks}

Das Orff-Schulwerk kann als ein offenes System beschrieben werden, zum einen in Bezug auf den Umgang mit den gedruckten veröffentlichten Modellen (Orff \& Keetmann, 1950-1954), zum anderen auf die Zielgruppe und auf neue Klangquellen sowie im Hinblick auf die Beziehung von Musik und Tanz, Interdisziplinarität und zeitgenössische Musik und Künste (vgl. Haselbach, 2013). Das Orff-Schulwerk umfasst eine Reihe von Prinzipien, die eine wichtige Rolle in seinem pädagogischen Konzept einnehmen (vgl. Haselbach, 2011; Haselbach \& Hartmann, 2013; Hartmann, 2017; Jungmair, 1992; Keller, 1974, 1996; Widmer, 2011):

- Der Mensch steht im Mittelpunkt: Das Orff-Schulwerk bietet in erster Linie kein spezialisiertes Training für Musik und Tanz, sondern eine Bereicherung der ganzen Persönlichkeit durch Erfahrungen und Ausdrucksmöglichkeiten in diesen Bereichen.

- Die soziale Dimension: Die Prozesse des Lernens, Arbeitens und Gestaltens werden vor allem in der Gruppe erfahren und fordern und fördern entsprechendes soziales Verhalten.

- Musik als integrales Konzept: In der griechischen Antike bedeutete das Wort musiké singen, tanzen, das Spielen von Instrumenten sowie die Verwendung von Sprache. Der Begriff musiké beinhaltet aber auch die verbindende Nähe zu anderen Kunstformen. Orff verstand dies als die Grundlage des Schulwerks.

- Die Anwendung von Instrumenten: Da körpergerecht gebaute Instrumente (sogenannte Orff-Instrumente) zur Verfügung gestellt werden, die ohne technische Hindernisse spielerisch erfahren werden können, ist die Möglichkeit gegeben, die Spielweise und deren Schwierigkeitsgrad an die Fähigkeiten der Spielenden anzupassen. Diese Instrumente sind sowohl für solistisches Spiel als auch für Gruppen geeignet, und zwar nicht nur zur Reproduktion von Stücken, sondern auch für Experimente, Improvisation und Komposition. Orff ermutigte zur ständigen Suche nach neuen Klangmöglichkeiten und geeignetem Material für elementares, körpernahes Musizieren.

- Prozessorientiertes Lernen: Die Schüler*innen werden auf kreative Weise in den Prozess eingebunden und bestimmen so Richtung und Resultat mit. Arbeitsprozess und künstlerisches Resultat haben die gleiche Wertigkeit.

- Kreativität in Improvisation und Komposition, in Musik und Tanz/Bewegung: Die Art des Unterrichtes gibt den Schüler( ${ }^{*}$ inne)n Gelegenheit, sich selbst als Gestaltende und Mitgestaltende zu erfahren. 
- Anwendbarkeit des Orff-Schulwerks: Das Orff-Schulwerk versteht sich selbst als ein offenes pädagogisches Konzept, das aufgrund seiner Prinzipien in allen Erziehungsbereichen anwendbar und auch auf verschiedene Kulturen übertragbar ist.

Aus meiner Sicht ist exzellente Pädagogik von Natur aus inklusiv: Grundlegende, kindzentrierte, inklusive Erziehung beinhaltet das Unterrichten von Kindern und Erwachsenen, die sich auf verschiedenen Entwicklungsebenen befinden und unterschiedliche Kompetenzen in Wahrnehmung, Kognition und Verhalten aufweisen. Sie erkennt die Individualität jedes Menschen (im Sinne ihrer oder seiner einzigartigen früheren Erfahrungen) und damit die Heterogenität jeder menschlichen Gruppe an (vgl. Feuser, 2001).

Eine Gruppe zu unterrichten beinhaltet, dass man sich über Faktoren wie individuelle Lernstile und Lernmodi, Lerntempi, Stand des Lernens wie auch Motivation und Konzentration (vgl. IQSH, 2011) der einzelnen Mitglieder bewusst ist. Die Lehrperson sollte sich darum bemühen, die Lernschritte für jedes Kind individuell zu planen und dessen Fortschritte festzustellen. Der Unterricht sollte alle Lernenden berücksichtigen und geeignete Strategien beinhalten, die das Lernen und Lehren unterstützen (vgl. Feuser, 1996; Goodkin, 2012). Durch eine Erweiterung und Verbesserung des Lernumfeldes - zu dem auch die anderen Schüler*innen mit ihren jeweiligen Kompetenzen beitragen können - kann eine positive Entwicklung eher erreicht werden als mit Unterrichtsmethoden, die darauf zielen, den Lernprozess auf Basis einer Defizitdiagnose zu beschleunigen (vgl. Athey, 1990, S. 76).

In der eingangs erwähnten Kindergruppe war es notwendig, Sicherheit und Vertrauen wie auch Selbst-Bewusstsein und Selbst-Vertrauen aufzubauen. Dies wurde vor allem durch einen ritualisierten Stundenablauf, die positive Atmosphärengestaltung, das Einschätzen und Eingehen auf die aktuelle Beziehungsqualität sowie die Unterstützung kleinster Lernschritte ermöglicht. „Wer kreativ sein will, braucht [...] Vertrauen in sich selbst, in seine eigenen Fähigkeiten und Fertigkeiten, in die eigenen Erfahrungen und das eigene Wissen." (Hüther, 2008, S. 122). Der Neurobiologe Gerald Hüther (2008) unterstreicht die Wichtigkeit von Sicherheit und Vertrauen - auch für kreative Prozesse. Weiters führt er aus, dass jede Person neue Wahrnehmungen und Erfahrungen mit bereits Bekanntem zu verbinden versucht. Die Bereitschaft etwas Neues auszuprobieren hängt davon ab, wie sicher man sich seiner selbst ist, wieviel Vertrauen entstanden ist, sich mit der Welt zu konfrontieren und wie bereits vorhandene Repräsentationen im Gehirn Konnexe mit aktuellen Erfahrungen bilden können. Sofern Unsicherheit, Angst oder Druck auf die Person einwirken, können die durch die Sinne aufgenommenen Wahrnehmungsmuster 
nicht mehr mit abgespeicherten Erinnerungen (Repräsentationen) verglichen und verknüpft werden und somit keine neuen Lernerfahrungen gemacht werden.

Für die Lehrperson bedeutet dies zuallererst, Aufgaben und Methoden zu finden, bei denen die Kinder sich sicher fühlen und bei denen ihre Mitarbeit geschätzt wird, damit sie das Selbstvertrauen entwickeln können, etwas Neues zu versuchen. Das Wiederholen von Aufgaben, das Finden von Erweiterungen und Varianten ist ebenso wichtig wie die Fähigkeit der Lehrperson, spontane und ungeplante Reaktionen (mit Stimme, Instrument oder Bewegung) zu beobachten und aufzugreifen.

In jeder Gruppe kann soziales Lernen auf zweierlei Weise gesehen werden: Erstens geht es darum, ein besseres soziales Verhalten zu erlernen und zweitens darum, auf eine soziale Weise zu lernen, indem das Erlernen beispielsweise eines Liedes, Tanzes oder Textes auf eine gemeinschaftliche Weise mit der Hilfe anderer geschieht. Das Spektrum der individuellen Fähigkeiten in einer Gruppe sollte nicht als Schwierigkeit, sondern als wichtiges Mittel für Motivation, Stimulation und Unterstützung für jedes Individuum gesehen werden.

Einer der wichtigsten Beiträge des Orff-Schulwerks kann darin gesehen werden, soziales Lernen in verschiedenen sozialen Konstellationen zu ermöglichen. Hier können Begegnungen auf unterschiedlichen Ebenen und vielfältige Art geschehen. Durch geschicktes Vorgehen können soziale Resonanz und Feingefühl bei allen Teilnehmer $\left({ }^{*}\right.$ inne)n gefördert werden, unabhängig von Begabungen oder Bedürfnissen. Unterschiedliche soziale Gruppierungen bilden einen wichtigen Aspekt in der Arbeit mit dem Orff-Schulwerk. Das Singen, Tanzen, Spielen von Instrumenten und Sprechen von Texten in der ganzen Gruppe wird normalerweise in jeder Stunde ergänzt durch Einzelaufgaben, Partner- und/oder Kleingruppenarbeit. Gruppenarbeit - sei es beim Üben vorgegebener Aufgaben oder bei der Findung eigener Lösungen - bezieht jede und jeden innerhalb der Gruppe mit ein, beugt Isolierung, Trennung und Ausschluss vor und stärkt die Gemeinschaft. Je größer die Vielfalt der Möglichkeiten in der Gruppe ist, was natürlich ein differenziertes Unterrichten voraussetzt, umso mehr Möglichkeiten sozialen Lernens entstehen.

Unterricht im inklusiven Sinn bedeutet nicht, jede Unzulänglichkeit als Behinderung zu analysieren. Es geht vorrangig darum, eine geeignete Didaktik $\mathrm{zu}$ verwenden und nicht um Fähigkeiten und Schwierigkeiten eines besonderen Kindes (vgl. Feuser, 2006). Nach Feusers Prinzipien einer inklusiven Erziehung sollte Unterricht alle Kinder einer Klasse oder Gruppe einbeziehen und dabei ihre individuellen Fähigkeiten, Interessen und Bedürfnisse berücksichtigen. 
„Inklusion verweist im Feld von Erziehung, Bildung und Unterricht über alle Lebensalter hinweg auf eine Pädagogik, die es ermöglicht, dass alle [...] in Kooperation miteinander, auf ihrem jeweiligen Entwicklungsniveau, nach Maßgabe ihrer momentanen Wahrnehmungs-, Denk- und Handlungskompetenzen, in Orientierung auf die nächste Zone ihrer Entwicklung, an und mit einem Gemeinsamen Gegenstand, spielen, lernen, studieren und arbeiten." (Feuser, 2015, S. 45)

In der Verwirklichung inklusiven Unterrichts sind die zwei Faktoren Individualisierung und Miteinander wesentlich. Elementare Musik- und Tanzerziehung, wie sie in dieser Kindergruppe angeboten wird, bietet einen Unterricht, der jedes einzelne Kind in den Fokus rückt. Innere Differenzierung wird möglich, wenn die Lehrperson jedem Kind erlaubt, das Thema auf seine eigene Weise $\mathrm{zu}$ verstehen und zu erproben und wenn individuelle Aufgaben gestellt werden, die gleichzeitig im Gesamtthema oder Projekt eine Bedeutung haben.

\section{Spiellieder}

Spiellieder bieten viele Möglichkeiten für Vorschul- und Grundschulkinder und sind besonders geeignet für inklusive Gruppen. In einem multisensorischen Ansatz wie dem Orff-Schulwerk können Spiellieder motivieren, ermutigen und zu einer Vielfalt von Tätigkeiten anregen, die Musik, Bewegung, Sprache, Spielmaterial und Objekte einbeziehen. Mehrere Zielsetzungen können mit diesen Tätigkeiten geplant und durchgeführt werden, um sich auf unterschiedliche Bedürfnisse, Interessen und Fähigkeiten zu konzentrieren. Spielen, sich bewegen, sprechen, singen, tanzen und etwas gestalten kann in einer Vielfalt von Möglichkeiten erfahren werden. Dieser multisensorische Ansatz bietet ein breites Spektrum an Erfahrungen und Ausdrucksmöglichkeiten, welche die Gesamtentwicklung der Kinder fördern (vgl. Salmon \& Stelzhammer-Reichhardt, 2008)

Spiellieder können zum Ausgangspunkt für Aktivitäten werden, die dem Entwicklungsstand und den Bedürfnissen jedes Kindes entsprechen (vgl. Salmon, 2014). Das Anregen und Inspirieren durch Lieder, Bewegung, Tanz, Rollenspielen, Sprachspielen, emotionalem Ausdruck und Fantasie sind wichtige Teile des Unterrichts. Die Lehrperson bietet eine Vielfalt von zentralen Bereichen an (siehe Abb. 1), wie bspw. Bewegungsgestaltung, sensorische Sensibilisierung, Umgang mit Körperinstrumenten, die im jeweiligen Spiellied enthalten und auch einzeln wirksam sind, umso mehr aber, wenn sie miteinander verbunden werden - besonders in Gruppen mit unterschiedlichen Voraussetzungen. 
Zusätzlich kann die Betonung einzelner oder mehrerer Elemente sowohl in der Planung von Einzelstunden wie auch von längerfristigen interdisziplinären Projekten dienlich sein. Das Lied selbst kann motivieren, anregen und in den einzelnen Bereichen (siehe Abb. 1) ausgebaut werden. Die endgültige Auswahl von Aktivitäten steht im Zusammenhang mit dem Inhalt des Liedes sowie mit den momentanen Fähigkeiten und Bedürfnissen der Kinder und der Gruppe (vgl. Salmon, 2006).

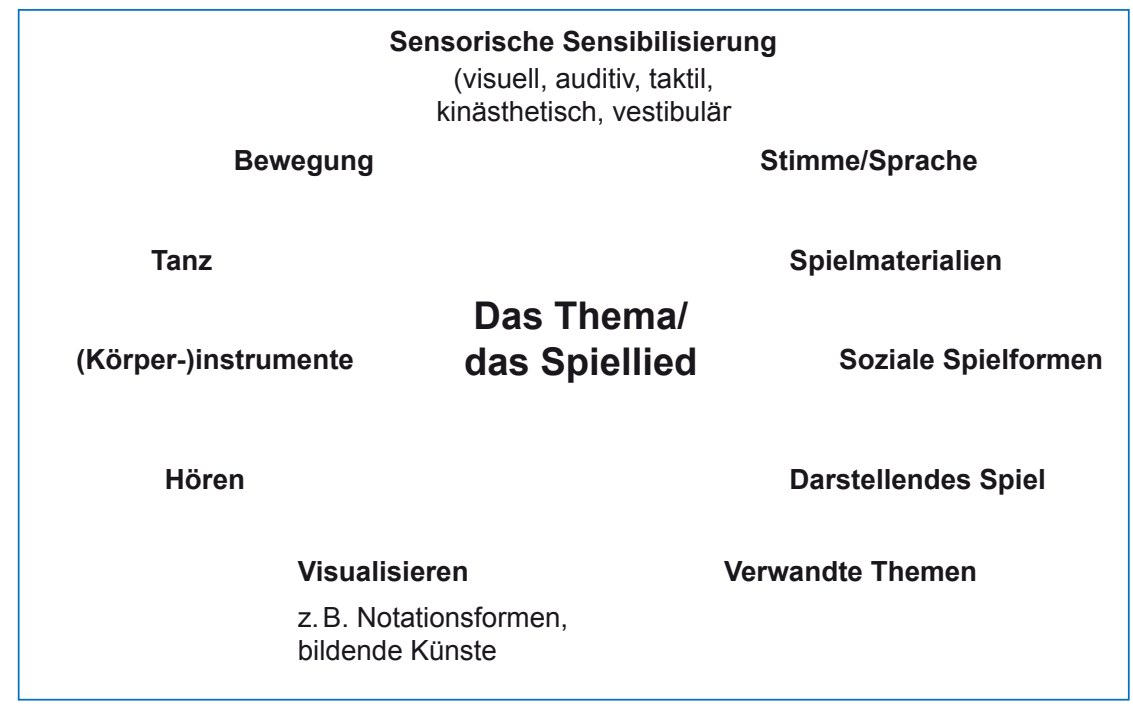

Abbildung 1: Mögliche Gestaltungsschwerpunkte eines Themas oder Spielliedes

Zeit und Raum zu spüren, damit zu spielen, zu experimentieren und zu gestalten ist unerlässlich in der Elementaren Musik- und Tanzerziehung, ganz besonders, wenn man mit Spielliedern arbeitet. Ein gewisses Quantum an Freiheit ist nötig, damit die Teilnehmenden ihre Freude und Leidenschaft für spontanes Spiel (wieder-)entdecken und vertiefen können. Spielraum und Stunde sollen eine spielerische Atmosphäre haben, vertraut und einladend sein. Raum zum Spielen anzubieten bedeutet aber nicht totale Freiheit. Das Akzeptieren allgemeiner Regeln ist unerlässlich für das Erkunden und Spielen in einer Gruppe (vgl. Jungmair, 1992). Das Zeitgefühl ist individuell sehr unterschiedlich, besonders in heterogenen Gruppen. Kinder brauchen Zeit, um ihre Tätigkeit zu entwickeln und sich darin zu vertiefen, Zeit, in der sie allein, mit einem Partner oder einer kleinen Gruppe experimentieren können und vor allem Zeit für Flow-Erlebnisse (vgl. Csikszentmihalyi, 1990). 
Divergentes Denken (vgl. Siegenthaler \& Zihlmann, 1988) ist besonders bedeutungsvoll in Gruppen mit unterschiedlichen Begabungen. So kann der ganzen Gruppe eine Aufgabe gestellt werden, die viele unterschiedliche Lösungen zulässt. Obwohl Kinder vielleicht Hilfe benötigen, um die Aufgabe zu verstehen, sagt ihnen die Lehrperson dennoch nicht, welche Lösung sie finden sollen. Jedes Kind findet seine Lösung mit den Fähigkeiten, die es zurzeit zur Verfügung hat, die verschiedenen Lösungen werden nicht miteinander verglichen und jeder Beitrag wird als wertvoll anerkannt.

Für Wilhelm Keller (1996) ist das Aufwerten von Nebenrollen in Gruppen unterschiedlicher Begabungen wichtig: Einer einfachen Handlung wird zentrale Bedeutung gegeben. Kinder, die nicht rhythmisch begleiten oder spielen, aber auf ihren Instrumenten den Eindruck des durch die Bäume pfeifenden Windes wiedergeben können, bekommen diese improvisatorische Aufgabe in einem Rondo, während ihre Freunde singen oder Begleitungen spielen. In der Improvisation bringt jede Person das ein, was im gegenwärtigen Zeitpunkt ihrer Entwicklung möglich ist. Während einige Schüler*innen vielleicht fähig sind, eine Melodie in einer Fünfton-Skala zu erfinden, zu erinnern und aufzuschreiben, mögen andere ihre Melodie auf den vorgegebenen Tönen spielen und wieder andere vielleicht einen Bordun dazu spielen (vgl. Salmon, 2014).

\section{Exemplarische Ideensammlung anhand des Uhu-Liedes von Gerda Bächli}

Gerda Bächli (1921-2013) war eine Schweizer Musiklehrerin und Komponistin von Liedern für Kinder jeden Alters und aller Begabungen. Bächlis Vorstellung vom Eulenlied (siehe Abb. 2) ist, dass jede Nacht, wenn es dunkel ist und alle Menschen schlafen, eine Eule mit ihren großen Flügeln durch die Stadt fliegt. Wenn sie einen Kirchturm berührt, beginnen die Glocken zu klingen (vgl. Bächli, 1977). Bächli definiert folgende Ziele für den Umgang mit diesem Spiellied: Die geheimnisvolle nächtliche Atmosphäre beruhigt viele Kinder, gefällt ihnen und ermutigt sie, dem improvisierenden Kind zu lauschen. Durch die Stille, in der sie mit geschlossenen Augen auf die Eule warten, sind die Kinder oft aufnahmefähiger (vgl. Bächli, 1977). 


\section{Das Uhu-Lied}

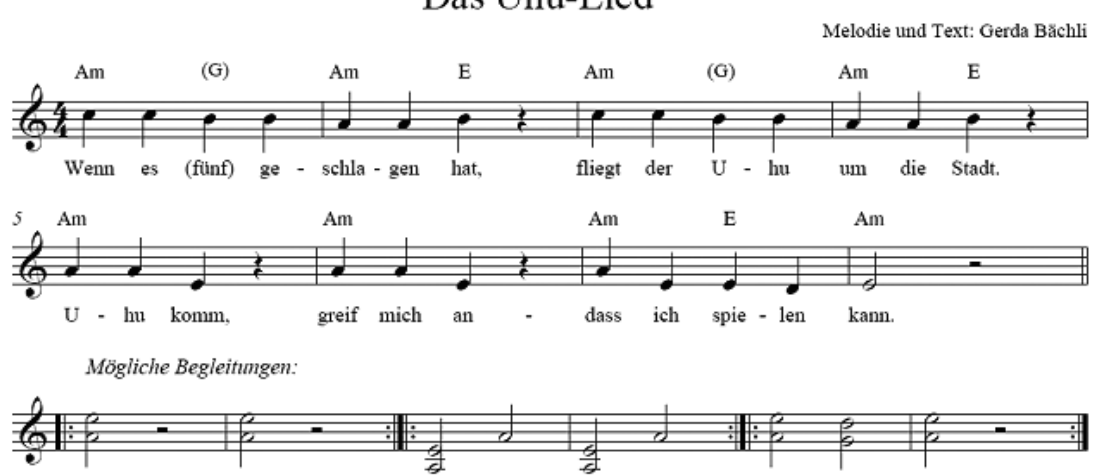

Abbildung 2: Uhu-Lied (Bächli, 1977)

\section{Unterrichtsplanung}

Die Planung für die eingangs beschriebene Gruppe umfasst eine Sequenz von Stunden mit mehreren Gestaltungsschwerpunkten (siehe Abb. 1) und beinhaltet Überlegungen zu:

- musikalischem Material (Melodie, Rhythmus, Harmonie, Form, Tonart)

- Text

- außermusikalischen Themen wie bspw. Handlung, Inhalt, Geschichte, Schauplatz, Atmosphäre, Bilder, Requisiten

- den anzusprechenden Intelligenzbereichen nach Howard Gardner, wie z. B. dem linguistischen, logisch-mathematischen, räumlichen, musikalischen, kinästhetischen, interpersonalen, intrapersonalen oder naturalistischen Intelligenzbereich (vgl. Gardner, 1993)

- unterschiedlichen Medien (Bewegung, Tanz, Stimme, Instrumente, bildnerisches Gestalten)

- unterschiedlichen Aktivitätsformen, wie Wahrnehmen, Entdecken, Experimentieren, Spielen, Erkennen, Erinnern, Imitieren, Variieren, Beschreiben, Differenzieren, Erfinden, Entscheiden, Üben, Mitteilen, Gestalten

- sozialen Aspekten und Möglichkeiten für Zusammenarbeit und Interaktion

- Vorerfahrungen und Entwicklungsstand der Kinder und individuellen Lernzielen 


\section{Aktivitäten}

Die im Folgenden beschriebenen Tätigkeiten sind als eine Ideensammlung gedacht und können in sämtlichen Gruppen eingesetzt werden. Sie sind nicht hierarchisch geordnet und bieten einen vielseitigen musikalischen Umgang mit diesem Lied über mehrere Stunden. Bei vielen dieser Beispiele muss das Lied mehr als einmal gesungen werden - manchmal nur die Melodie auf Vokalise ohne Text. ${ }^{3}$

In der Praxis werden oft mehrere dieser zentralen Gestaltungsschwerpunkte miteinander verbunden und entsprechen einem oder mehreren der Intelligenzbereiche. Innerhalb eines Bereichs können individuelle Aufgaben auf unterschiedlichem Niveau gestellt werden. Ein Kind, das schon ein Instrument beherrscht, bekommt entsprechend seiner Fähigkeit die Aufgabe, die Melodie zu spielen, zu improvisieren oder einen zweiten Teil zu erfinden. Wieder andere Kinder spielen verschiedene Begleitungen auf kleinem Schlagwerk oder Stabspielen. Einem Kind macht es z. B. Freude, Instrumente zu spielen. Es kann aber noch keine Ostinati imitieren. So darf es als Einleitung oder Zwischenspiel bestimmte Effekte erzeugen (z. B. den Wind oder das Geräusch, das die Flügel des Uhus machen).

Es gibt viele Möglichkeiten, Lieder einzuführen - hier begannen wir mit einer Uhu-Handpuppe, welche die Kinder musikalisch begrüßte, die man streicheln durfte und die den Kindern bestimmte Bewegungen vormachte.

\section{Sinnesschulung}

- Mit geschlossenen Augen dem Uhu lauschen (die Lehrerperson bewegt sich im Raum) und zeigen, woher das Geräusch kommt.

- Anderen nächtlichen Geräuschen im Wald, die von der Lehrperson oder auch einigen Kindern gespielt werden, lauschen (Rascheln, Wind, Tiere).

- Ein Kind darf den Uhu-Flug mit einem Schal imitieren. Die anderen folgen diesem zunächst mit den Augen.

- Die Linie des Uhu-Flugs mit dem Finger auf den Boden zeichnen.

- Die Linie auf den Rücken eines Partners zeichnen.

- Die Arme zum imaginären Fliegen benutzen. Mit geschlossenen Augen am Platz fliegen.

- Einen Baum darstellen (sitzend, kniend oder stehend). Leichtes Schwanken im Wind, dabei die Balance halten.

- Die Linie der Melodie mit einer Hand (oder beiden Händen) in die Luft zeichnen.

3 Wenn die Melodie für manche Kinder zu hoch liegt, kann sie auch in einer anderen Tonart gesungen werden. 
Bewegung

- Während die Melodie einmal mit Wiederholung gespielt oder gesungen wird von seinem Nest wegfliegen und zum Ende der Melodie zurückgekehrt sein.

- Die Bewegung verschiedener Vögel mit Händen und Armen zu dieser oder einer anderen Musik imitieren.

- Verschiedene Raumwege fliegen (gerade Linien, Kurven, Kreise, Spiralen, Zickzack, Dreiecke).

- Kurze oder lange Strecken fliegen.

- Einem Partner auf seiner Fluglinie nachfliegen, Partnerwechsel.

- Gegenstände oder Kinder stellen Bäume dar. Der Uhu fliegt herum, ohne dabei anzustoßen (eventuell auch mehrere Uhus).

Tanz

- Bewegungen für verschiedene Vögel erfinden.

- Verschiedene Schritte vorwärts, rückwärts, seitwärts lernen und sie zur Melodie anwenden.

- Armhaltungen ausprobieren und zu den Schritten anwenden.

- Die Kinder erfinden in Paaren einen Tanz nur mit Händen und Armen.

- Die Kinder erfinden und üben in Paaren oder Kleingruppen eine einfache Tanzform zur Melodie.

- Einen Volkstanz zum Thema Vögel, Nacht oder Wald erlernen.

Stimme und Sprache

- Uhu-Geräusche erfinden, anderen zuhören und Ideen imitieren.

- Durch Verbindung von zwei verschiedenen Geräuschen eine kleine Geräuschfolge produzieren und einem anderen Kind beibringen.

- Die Melodie mit verschiedenen Tonsilben singen.

- Einen Text zu den Waldgeräuschen erfinden (oder den Liedtext variieren).

- Mit einem Uhu-Gedicht arbeiten.

Instrumente

- Mit verschiedenen Instrumenten unterschiedliche Effekte improvisieren (Wind, Flügelgeräusch, Glocken, Waldgeräusche etc.).

- Kirchenglocken mit einem selbstgewählten Instrument präsentieren und verschiedene Uhrzeiten (5 Uhr, 8 Uhr usw.) spielen (Triangel, Becken, Glocke usw.).

- Einfache Blasinstrumente vorstellen und damit experimentieren (Okarina, Flötenkopf, Lotusflöte etc.).

- Das Lied mit einem a-Moll-Bordun (A-E) auf Stabspielen begleiten.

- Mit schweifendem Bordun (A-E, G-D) begleiten. 
- Verschiedene rhythmische Ostinati erfinden oder imitieren: Uhu •- •- Mit-ter-nacht $\bullet \bullet \bullet-$, viele Bäume

- Die ersten zwei Takte des Liedes auf Stabspielen spielen lernen.

- Zweitaktige Melodieteile als melodische Ostinati wiederholen lernen.

- Das Lied auf der Gitarre begleiten (a-Moll abwechselnd mit G-Dur und/ oder $\mathrm{E}^{7}$ )
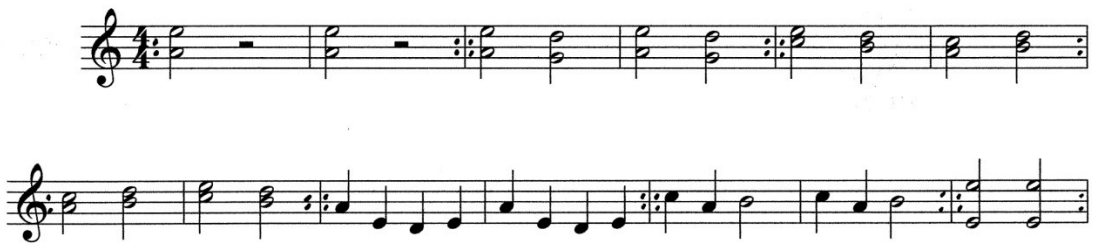

Abbildung 3: Beispiele für Ostinati

\section{Hören}

- Mit geschlossenen Augen erkennen, wo der Uhu sich befindet (die Lehrperson oder ein Kind spielt auf der Lotusflöte oder einem Blockflötenkopf). Zeigen, woher der Ton kommt.

- Mit geschlossenen Augen den Kirchenglocken lauschen. Wie viele Schläge sind zu hören?

- Die Tonhöhe einer Melodie mit der Hand nachzeichnen. Wann fliegt der Uhu hoch, wann tief?

- Partneraufgabe: Zwei Kinder wählen ein kleines Schlaginstrument oder eine Glocke, eines spielt, das andere lauscht mit geschlossenen Augen. Sobald der Ton endet, zeigt das lauschende Kind in die Richtung, aus welcher der Ton kommt (mehrmals versuchen, dann Rollentausch).

- Instrumente (kleines Schlagwerk, Klangstäbe, Glocken) werden im Raum verteilt, sie stellen die Häuser einer Stadt dar. Einige Kinder fliegen von Haus zu Haus und spielen die Instrumente. Wenn alle dran waren, wählt jedes Kind eines der Instrumente. Ein Kind fliegt als Uhu durch die Stadt, wenn es sich einem Haus (Instrument) nähert, erklingt das Haus (wird das Instrument gespielt).

- Ein Kind führt ein anderes, das seine Augen geschlossen hat, durch die Stadt. Wenn sie sich einem Haus nähern, ertönt das Haus.

- Musikstücke oder Teile von Kompositionen, die mit den Themen Vögel, Nacht, Kirchenglocken zu tun haben, werden angehört. ${ }^{4}$

4 Beispiele hierfür sind Blackbird (The Beatles), der Feuervogel (Strawinsky), Dies Irae aus der Symphonie Fantastique (Berlioz), La cathédrale engloutie (Debussy), Night on a Bare Mountain (Mussorgsky). 
Visualisieren

- Die Fluglinie des Uhus wird mit einer Hand in die Luft gezeichnet.

- Die Linie wird mit einem Pinsel (jedoch ohne Farbe) auf den Körper, auf den Boden oder auf ein großes Stück Papier gezeichnet.

- Die Linie wird mit Kreide, Wachskreiden oder Stiften auf Papier gezeichnet. Die Blätter werden getauscht und es wird versucht, der dort aufgezeichneten Fluglinie (in Form von Bewegungen) zu folgen.

- Verschiedenen Kirchenglocken lauschen und versuchen, die Glocke zu malen oder den Klang zu notieren. Jüngere Kinder malen wahrscheinlich verschiedene Glocken, ältere malen den Klang jeder Glocke.

- Die Anzahl der Glocken aufzeichnen, die von einem selbst oder einem Partner gespielt werden.

- Verschiedene Möglichkeiten der Notation einer Melodie erkennen. ${ }^{5}$

- Ein Bild mit einem Uhu, das die Lehrperson mitgebracht hat, betrachten. Was ist zu sehen? Wie könnte man eine Szene entwickeln und spielen?

Sozialformen

- Verschiedene Rollen darstellen (Uhu, Kirchtürme, Bäume).

- Ein Partnerkind (dessen Augen geschlossen sind) an der Hand im Raum herumführen.

- Ein Partnerkind (dessen Augen geschlossenen sind) durch das Spielen eines Instruments im Raum herumführen, ohne dass es irgendwo anstößt.

- Gemeinsam mit den anderen Kindern eine Nachtmusik improvisieren. Mit einem Partner oder in einer kleinen Gruppe Instrumente wählen und eine eigene Nachtmusik oder Uhu-Flugmusik erfinden und üben.

Elementares Musiktheater

- Eine Geschichte zum Lied erfinden.

- Mit Mitteln des Sprechens, der Bewegung, des Singens und Tanzens diese Geschichte darstellen.

- Ein geeignetes Kinderbuch zum Thema auswählen und damit eine elementare Musiktheater ${ }^{6}$-Produktion gestalten (vgl Widmer, 2004).

5 Z.B. Die Lehrperson zeigt den Verlauf der Melodie mit einer Hand. Die Kinder beobachten und versuchen, es selbst zu zeigen (auch mit geschlossen Augen). Die Lehrperson bereitet viele Karten vor, wo jeweils zwei Takte der Melodie grafisch notiert sind. Diese Karten werden durcheinander auf den Boden gelegt bzw. in Kleingruppen gegeben. Die Lehrperson singt zwei Takte des Liedes (ohne Text), die Kinder erkennen welche Karte dazu passt.

6 Das Elementare Musiktheater (EMT) entstand im Rahmen der Elementaren Musikund Tanzpädagogik am Orff-Institut der Universität Mozarteum Salzburg. Wilhelm Keller führte diese Spielform erstmals 1988 mit einer inklusiven Gruppe durch. Manuela Widmer hat das Elementare Musiktheater weitergeführt, didaktisch aus- 


\section{Zusammenfassende Gedanken}

Es ist für jede Art von Unterricht wesentlich, jede Einzelne und jeden Einzelnen in die Gruppe einzubeziehen, um die Teilnahme zu fördern und jedem Kind zu ermöglichen, auf seiner momentanen Stufe zu lernen. Das Orff-Schulwerk stellt das Individuum ins Zentrum und bietet Möglichkeiten für differenzierte Aufgabenstellung in der Arbeit an einem gemeinsamen Thema. Auf diese Weise unterstützt es inklusiven Unterricht für alle Altersgruppen und Begabungen. Es stellt eine hochinteressante, offene und flexible Möglichkeit dar, die von Seiten der Lehrendenpersönlichkeit eigene Fantasie, Flexibilität und kreative Fähigkeiten fordert. Im Ansatz des Orff-Schulwerks arbeiten Lehrende mit einem breiten Spektrum an Mitteln, sie erkennen das Lernverhalten jedes Kindes, können Inhalte vereinfachen oder ausdehnen, können auf unterschiedlichen Ebenen mit Musik und Bewegung gestalten und Kindern ermöglichen, auf ihrer eigenen Stufe von Geschicklichkeit und Verständnis zu spielen. Dieser Ansatz bedeutet auch, dass wir Lehrenden jeden Beitrag würdigen und den Kindern Gelegenheiten schaffen, auf musikalische Entdeckungsreise gehen zu können (vgl. Goodkin, 2012). Kinder brauchen die Möglichkeit, ihre Talente zu entdecken, ihre individuellen Ideen und Lösungen zu entwickeln, ihre musikalischen (rhythmischen, melodischen, harmonischen) und bewegungsmäßigen Aufgaben zu üben, ihren persönlichen Ausdruck und ihre Kreativität zu finden, sich in ihrem Sozialverhalten zu entwickeln und sich mit Musik und Tanz zu identifizieren.

\section{Literatur}

Athey, C. (1990). Extending thought in young children: A parent-teacher partnership. London: Paul Chapmann Publishing Ltd.

Bächli, G. (1977). Der Tausendfüßler. 2 × 11 Lieder für Vorschulkinder, Heimkinder, behinderte Kinder. Zürich: Musik Hug Verlage.

Csikszentmihalyi, M. (1990). Flow: The psychology of optimal experience. New York: Harper \& Row.

Feuser, G. (1996). Thesen zu: „Gemeinsame Erziehung, Bildung und Unterrichtung behinderter und nichtbehinderter Kinder und Jugendlicher in Kindergarten und Schule (Integration)“. Verfügbar unter: http://bidok.uibk.ac.at/library/feuserthesen.html [29.07.2020].

geleuchtet und verbreitet. Im EMT werden Musik, Tanz und Sprache miteinander verbunden. Im Mittelpunkt stehen die gemeinsame Spielfreude, das eigene Gestalten und der künstlerische Ausdruck (siehe mehr dazu unter: http://elementaresmusiktheater.com/was-ist-emt/ [29.07.2020]). 
Feuser, G. (2001). Prinzipien einer inklusiven Pädagogik. Behinderte in Familie, Schule und Gesellschaft. Verfügbar unter: http://bidok.uibk.ac.at/library/beh201-feuser-prinzipien.html [29.07.2020].

Feuser, G. (2006). „Alle Menschen werden Brüder...“. Zeit und Rhythmizität als Grundprozess des Lebens - und der Verständigung. In S. Salmon (Hrsg.), Hören - Spüren - Spielen. Musik und Bewegung mit gehörlosen und schwerhörigen Kindern (S. 45-60). Wiesbaden: Reichert.

Feuser, G. (2015). Zwei Prämissen für „Kooperation an einem gemeinsamen Gegenstand“. In Steirische Vereinigung für Menschen mit Behinderung (Hrsg.), Behinderte Menschen (S. 45-49), Nr. 4/5/2015. Graz: RehaDruck.

Gardner, H. (1993). Frames of mind: The theory of multiple intelligences. London: Fontana Press.

Goodkin, D. (2012). Kein Kind ausschließen - der Ansatz des Orff-Schulwerks für eine differenzierte Unterrichtsgestaltung. Orff-Schulwerk Informationen, 87, 3840. Verfügbar unter: https://2bfcb07a-e06d-4788-ac65-cc3b369ce211.filesusr. com/ugd/8289b4_9d5478592bc74e119cb56ddb3ecc97ce.pdf [29.07.2020].

Hartmann, W. (2017). Die Prinzipien des Orff-Schulwerks. Orff-Schulwerk HEUTE, 97, 23-24. Verfügbar unter: https://2bfcb07a-e06d-4788-ac65-cc3b369ce211. filesusr.com/ugd/8289b4_41502809eb3a4db1bc5e26ee71e2877d.pdf [29.07.2020].

Haselbach, B. (Hrsg.). (2011). Orff-Schulwerk Basistexte: Studientexte zu Theorie und Praxis des Orff-Schulwerks. Basic texts: Texts on theory and practice of OrffSchulwerk. Band 1, Volume 1. Mainz: Schott.

Haselbach, B. (2013). Orff-Schulwerk: Origins and development. In S. Hennessy (Hrsg.), Reflections on Orff-Schulwerk (S. 10-18). London: Schott Music.

Haselbach, B. \& Hartmann, W. (2013). Notes for the Convention of the Orff-Schulwerk Forum 2013. Unveröffentlicht: Salzburg.

Hofmarksrichter, K. (1962). Orff-Schulwerk in Taubstummeninstitutionen. In W. Thomas \& W. Götze (Hrsg.), Orff-Institut. Jahrbuch 1962. Mainz: B. Schott's Söhne.

Hüther, G. (2008). Die neurobiologischen Voraussetzung für die Entfaltung von Neugier und Kreativität. Verfügbar unter: https://www.gerald-huether.de/pdf/ neurobiological_preconditions.pdf [29.07.2020].

IQSH Institut für Qualitätsentwicklung an Schulen Schleswig-Holstein (Hrsg). (2011). Umgang mit Heterogenität im Musikunterricht. Band 1. Kronshagen.

Jungmair, U. (1992). Das Elementare: Zur Musik- und Bewegungserziehung im Sinne Carl Orffs Theorie und Praxis. Mainz: Schott Music.

Keller, W. (1971). Das Orff-Schulwerk als musikalische Lebenshilfe. In H. Wolfgart (Hrsg.), Das Orff-Schulwerk im Dienste der Erziehung und Therapie behinderter Kinder (S. 3-17). Berlin: Marhold.

Keller, W. (1974). Ziele und Aufgaben des Instituts für Musikalische Sozial- und Heilpädagogik am Mozarteum in Salzburg. Orff-Schulwerk Informationen, 13. 
Keller, W. (1984). Elementare Musik von und mit Behinderten. Musik und Bildung, 16 (12), 797-802.

Keller, W. (1996). Musikalische Lebenshilfe. Ausgewählte Berichte über sozial- und heilpädagogische Versuche mit dem Orff-Schulwerk. Mainz: Schott Music.

Orff, C. (1964). Das Schulwerk - Rückblick und Ausblick. In W. Thomas \& W. Götze (Hrsg.), Orff-Institut Jahrbuch 1963 (S. 13-20). Mainz: Schott.

Orff, C. \& Keetmann, G. (1950-1954). Orff-Schulwerk. Musik für Kinder 1-5. Mainz: Schott's Söhne.

Orff-Schulwerk Heute Nr. 99 (2018). Orff-Schulwerk mit besonderen Zielgruppen im pädagogischen und sozialen Kontext.

Salmon, S. (Hrsg.). (2006). Die Bedeutung von Spielliedern in inklusiver Unterrichtsgestaltung. In S. Salmon (Hrsg.), Hören Spüren Spielen. Musik und Bewegung mit gehörlosen und schwerhörigen Kindern (S. 235-256). Wiesbaden: Reichert.

Salmon, S. (2012). MUSICA HUMANA - Gedanken über humanistische Aspekte des Orff-Schulwerks. Orff-Schulwerk Informationen, 87, 19-22. Verfügbar unter: https://2bfcb07a-e06d-4788-ac65-cc3b369ce211.filesusr.com/ugd/8289b4_9 d5478592bc74e119cb56ddb3ecc97ce.pdf [29.07.2020].

Salmon, S. (2014). Hallo Kinder. Lieder und begleitende Aktivitäten für Kinder von 4-9. Mainz: Schott.

Salmon, S. \& Stelzhammer-Reichhardt, U. (2008). „Schläft ein Lied in allen Dingen ..."Musikwahrnehmung und Spiellieder bei Schwerhörigkeit und Gehörlosigkeit. Wiesbaden: Reichert.

Siegenthaler, H. \& Zihlmann, H. (1988). Rhythmische Erziehung. Hitzkirch: Comenius-Verlag.

Widmer, M. (2004). Spring ins Spiel: Elementares Musiktheater mit schulischen und außerschulischen Gruppen. Ein Handbuch mit CD. Koblenz: Fidula.

Widmer, M. (2011). Die Pädagogik des Orff-Instituts: Entwicklung und Bedeutung einer einzigartigen kunstpädagogischen Ausbildung. Mainz: Schott Music.

Wolfgart, H. (Hrsg.). (1971). Orff-Schulwerk im Dienst der Erziehung und Therapie behinderter Kinder. Festschrift zum 75. Geburtstag von Carl Orff. Berlin: Marhold.

Wolfgart, H. (Hrsg.). (1975). Orff-Schulwerk und Therapie. Therapeutische Komponenten in der elementaren Musik- und Bewegungserziehung. Berlin: Marhold. 


\section{The Art of Inclusion: Modellierung polyästhetischer Ansätze in der Inklusion}

\section{Einleitung}

Ausgehend von der Frage, wie Kunst derzeit an der Intersektion zu Behinderung verhandelt, diskutiert und beforscht wird, möchten wir uns anhand eines - mit und in Schulen erarbeiteten - Modells an eine kritische Erweiterung dieses künstlerisch-pädagogischen Zugangs annähern: Polyästhetik, Inklusion und Schule. Um Kunst inklusiv zu rahmen, wollen wir eine breitere bzw. radikalere Perspektive, als diese an der Schnittmenge zwischen Kunst- und Behinderung bisher gedacht wird, einführen. Wir würden die Rahmung der bisherigen Perspektive wie folgt zusammenfassen:

- Einseitige Perspektive, z. B. Reduktion des Kunstschaffens von Menschen mit Behinderungen in Tagesstruktur als Form der Beschäftigungstherapie, Theaterprojekte für Menschen mit spezifischen Formen der Behinderung

- Folgende Aspekte unterscheiden unseren Ansatz von der klassischen Schnittmenge Behinderung und kunstgeleitete Aktivitäten:

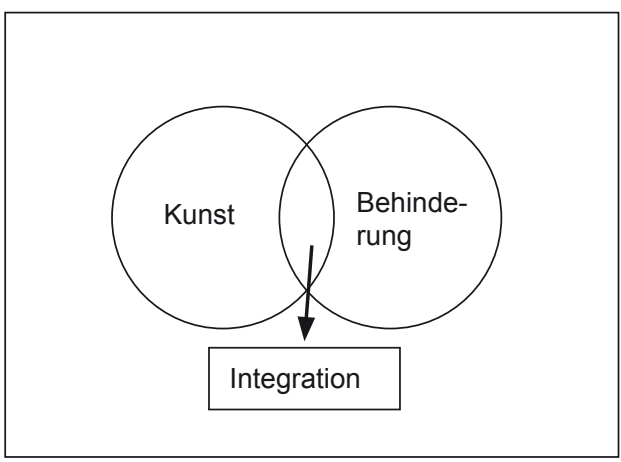

Abbildung 1: Schnittmenge Kunst und Behinderung

In Abbildung 1 wird die Intersektion Kunst und Behinderung verdeutlicht. An der Schnittmenge entstehen integrative Angebote und Möglichkeiten für Menschen mit Behinderungen.

In unserem Ansatz (s.o.) handelt es sich demgegenüber um keine therapeutische Mediation (vgl. Brun, Chouvier \& Roussillon, 2013) oder künstlerische Intervention von Künstler( ${ }^{*}$ inne $) n$ für ein bestimmtes Projekt. 
Bildende Kunst, Tanz und Musik werden auch als Medium in dem therapeutischen Prozess der Kunsttherapie, Tanztherapie oder Musiktherapie verwendet. Unser Zugang (s.o.) unterscheidet sich von Kunsttherapie, die laut Menzen wie folgt definiert werden kann: „Ihr Mittel besteht darin, jenen psychischen Ausdrücken, jenen Bildern, Vorstellungsmustern, die Leiden verursachen, eine andere Ausrichtung zu geben. Im Ergebnis sollen die Bewusstseinsund Erlebnisweisen, aber auch die Verhaltensabläufe mit bildnerischen Mitteln so konstelliert werden, dass es möglich wird, das Alltagsleben neu zu sehen und zu bewältigen." (Menzen, 2009, S. 22-23). Auch die Tanztherapie, die wie folgt definiert wird, unterscheidet sich von diesem Ansatz: „Tanztherapie versteht sich als die psychotherapeutische Verwendung von Tanz und Bewegung zur Integration von körperlichen, emotionalen und kognitiven Prozessen des Menschen. Sie wird auch als künstlerische Therapie definiert, die Tanz und Bewegung als Medium zur Persönlichkeitserweiterung nutzt.“ (BTD, 2020, S. $1^{1}$ )

- Menschen mit Behinderungen als Künstler*innen, die nicht als gleichwertige Kunstschaffende wahrgenommen werden. Ein Tanzstück wird in diesem Verständnis also besucht, weil hier professionelle Kunstschaffende mit Anderen arbeiten und dies in einem karitativen Zugang zu Behinderung als wohltätig betrachtet werden kann, wenn man eine derartige Veranstaltung besucht.

- Im schulischen Kontext wird Kunst meist unter einer kompetitiven bzw. ableistischen Perspektive vermittelt. So zieren die schönsten Bilder die Gänge der Schule oder jene derer, die etwas trotz ihrer Behinderung schaffen konnten.

Kunst wird in diesem Sinne im Kontext von Behinderung selten ebenbürtig zu Kunst von und mit Menschen ohne Behinderung und noch weniger als Akt der Selbstdarstellung und damit politisches Statement verstanden (vgl. Eisenhauer, 2007, S. 13). Behinderung nimmt der Kunst gegenüber eine Sonderstellung ein, die nicht inklusiv, in den seltensten Fällen vielleicht als integrativ, beschrieben werden kann. Dieser Umstand wird in Abbildung 2 illustriert, welche Abbildung 1 um ein inklusives Kunstverständnis ergänzt.

1 Für weitere Informationen siehe: https://www.btd-tanztherapie.de [16.06.2020]. 

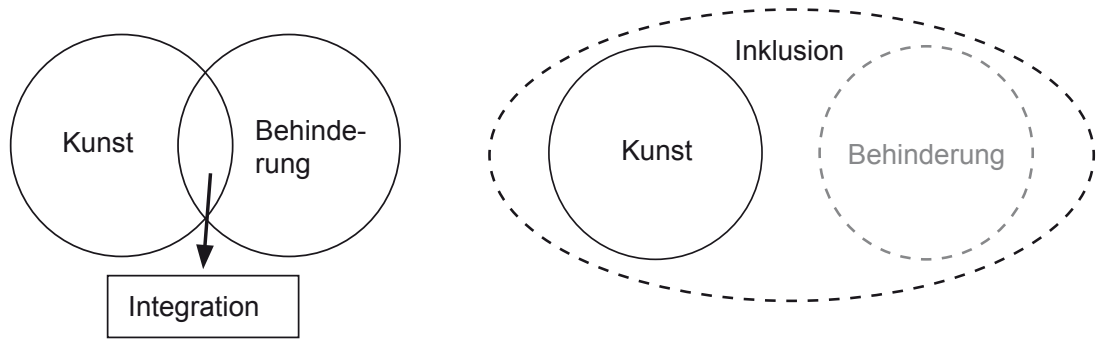

Abbildung 2: Kunst als einseitige Schnittmenge mit Integration (links) und Annäherung an ein inklusives Kunstverständnis (rechts)

Im Verlauf des Artikels wird die Verschränkung diverser künstlerischer Ansätze - tänzerisch, bildnerisch, musikalisch und wissenschaftlich-rahmend - dargestellt und über die Illustration eines sich im Entstehungsprozess befindenden Modells erläutert. Dabei wird das Verständnis von Kunst und Inklusion (im schulischen Kontext) erweitert. Vor dem Hintergrund der 2011 in Österreich eingeleiteten Reform der Lehrer(*innen)bildung, die auch eine Fokussierung auf Inklusive Pädagogik für alle Pädagog $\left({ }^{*}\right.$ inn)en vorsieht, bedarf es innovativer Konzepte, die Inklusion ermöglichen und aktiv dabei unterstützen, ein Bildungssystem für alle zu schaffen. Dies kann nur auf uneingeschränkter Chancengleichheit basieren.

Über die Annäherung durch Musik, Tanz und das gemalte Bild kann Raum in seiner inklusiven Qualität neu erfahren werden. Dieser polyästhetische Ansatz eröffnet durch die parallele gleichzeitige Anwendung veränderte Wahrnehmungen und Beziehungen.

Die zentrale Intention der polyästhetischen Methode ist die Entwicklung eines auf zeitgenössischem Tanz, rhythmisch-musikalischer Body Percussion und bildendem Gestalten basierenden, also polyästhetischen, Workshop-Konzepts zur Raumerfahrung und -gestaltung für Schüler*innen mit und ohne Behinderung, mit und ohne sogenanntem Migrationshintergrund und über klassische Altersgrenzen hinweg. ${ }^{2}$

2 In unserem Projekt agieren Schüler*innen im Alter von 6-14 Jahren gemeinsam über unterschiedliche Schulformen hinweg. 


\section{Theoretische Grundlagen: polyästhetische Annäherung an Raum und Inklusion}

Der Begriff Raum ist ein weit generalisierter Begriff, der verschiedene Repräsentationen und Definitionen je nach Fachbereichen beinhaltet. Er ist nicht nur geometrisch zu verstehen, wie der Philosoph Franz Xaver Baier erwähnt, sondern auch auf phänomenologischer Ebene und ebenfalls aus der Dimension der Architektur, der Kunst und anderen verschiedenen Lebensbereichen zu betrachten: „Wir leben in einer Vielfalt unterschiedlich konstruierter Räume, in denen unsere Existenz jeweils eine andere ist. Die unterschiedlichen Lebens-Räume bilden Zusammenhänge, die erst ein, nicht nur menschliches, Zusammenleben ermöglichen." (Baier, 2000, S. 7)

„Räume sind durch die sinnliche Erfahrbarkeit von Grenzen, etwa in Form von Linien, Umrissen und Grenzmarkierungen erfahrbar." (Heule, 2009, S. 5)

„Denn erst durch die Wahrnehmung einer Grenze konstituiert und differenziert sich ein Raum oder ein Gegenstand, schält sich ein Objekt aus seiner Umgebung heraus, wird unterscheidbar und damit wahrnehmbar." (Ehlers, 2007, S. 5) Wie ein Raum wahrgenommen wird, ist abhängig von den darin handelnden Personen, denn erst durch die Handlung erhält er seine spezifische Bedeutung. In welcher Beziehung die handelnden Personen stehen beeinflusst dementsprechend das Raumerlebnis. Zu berücksichtigen ist außerdem, wie ein Raum wahrgenommen wird (von den Schüler( ${ }^{*}$ inne)n wurden dem Raum zum Beispiel folgende Begrifflichkeiten zugeordnet: Wunschraum, Spielraum, Zwischenraum, Kommunikationsraum, Bewegungsraum usw.) und mit welchem Wesensmerkmal und unterschiedlichen Qualifikationen (zum Beispiel Ermöglichung von Freiraum, Ruhe, ein Ort der Gemeinschaft) er belegt ist. Ein Aneignungsprozess im Umgang mit den Räumen ${ }^{3}$ bedarf unterschiedlicher Fähigkeiten und Fertigkeiten, die in der entwickelnden polyästhetischen Methode angelegt und verankert sein werden. In einem handelnden Aneignungsprozess agieren die Schüler ${ }^{\star}$ innen mit ästhetischen Mitteln.

„Räume werden nicht passiv wie in einem Spiegel eingefangen. Jedes Individuum erschließt sich aktiv und individuell verschieden den es umgebenden Raum. Der Raum ist nicht allein visuell vor uns ausgebreitet, wir treten in nicht nur den einen Raum ein, sondern in verschiedene Räume, wir bewe-

3 Diese Prozesse umfassten zum Beispiel verschiedene Ideen, wie ein Raum mit Hilfe von unterschiedlichen Materialien (Klebeband oder bunten Bändern etc.) vermessen und in räumliche Abschnitte eingeteilt werden kann. In diesen unterschiedlichen Segmenten konzipierten Teams von Schüler( ${ }^{*}$ inne)n ihre im vorigen Workshop entstandenen Ideen zur Frage, was Raum eigentlich ist (z. B. Wunschraum, Spielraum etc.). Die von den Schüler $\left({ }^{*}\right.$ inne)n dafür verwendeten Methoden waren Body Percussion, Bewegung und Zeichnen. 
gen uns darin und erleben Räume. Wir können sie riechen, hören und fühlen und unterscheiden in Korrespondenz zu unserer Körperlichkeit, aber auch zu unseren Erinnerungen und Vorstellungen.“ ( Heule, 2009, S. 6) „So unterscheidet sich auch der eigene Körper als Raum, dessen Grenze die eigene Haut ist, von anderen Körpern mit ihren jeweils eigenen Grenzen." (Ehlers, 2007, S. 5)

Dieser polyästhetische Ansatz eröffnet durch die parallele gleichzeitige Anwendung von unterschiedlichen Kunstformen veränderte Wahrnehmungen und Beziehungen. Der Musiktherapeut und Dozent für Improvisation Fritz Hegi spricht von Klang-Atmosphären, die einen Raum verwandeln. „Klingende Räume und gefühlte Klänge verschmelzen zum Unbestimmten von Atmosphären und verwandeln sich - verwandeln uns - in andere Stimmungen. Sie bestimmen einen Raum, ändern ein Gefühl, fangen an zu klingen." (Hegi, 2005, S. 72)

Es geht in unserem Modell darum, Kindern und Jugendlichen Raum zu geben, gemeinsam Raum zu erleben, Wunschräume zu entwerfen und Bedeutungsräume zu schaffen. Handlungsräume und Räume, in denen Grenzerfahrungen möglich sind, ermöglichen es, sich auf Improvisation einzulassen, eine reale Raum-Zeitreise anzutreten. Dazu schreibt Elisabeth Gutjahr: „Jeder Augenblick entscheidet über den nächsten, alles ist Bewegung und aufeinander beziehbar. In Erwartung des Unvorhergesehenen sind Aufmerksamkeit und Reaktionsbereitschaft hoch. Vieles geschieht gleichzeitig und durchdringt sich.“ (Gutjahr, 2014, S. 21)

Die Erfahrungen der Kinder und Jugendlichen, wie auch der Lehrer*innen und weiterer Stakeholder im Feld (Künstler*innen, Studierende etc.) fließen in die Aus- und Weitergestaltung des Workshop-Konzepts ein. Die Gestaltung ist im Sinne partizipatorischer Forschung eingebettet, wird durch diese begleitet, gerahmt und erweitert (vgl. Nind, 2014).

In unserer Wahrnehmung der modularen Annäherung an ein inklusives Kunstverständnis, das gemeinsam mit Lernenden entwickelt wird, sind Kunst und Kultur unverzichtbare Anteile einer umfassenden Bildung, die das Wohl jedes einzelnen Menschen, seine bestmöglichen Entfaltungen und seine aktive Teilnahme am gesellschaftlichen Leben als gestaltendes Mitglied einer Gemeinschaft zum Ziel hat. Kunst kann, ähnlich der Wissenschaft, durch ihren Blick auf die Welt und ihren Zugang zur Kreativität zu einem gesamtgesellschaftlichen Entwicklungsprozess führen. Daher wird Kreativität in diesem Kontext als Fähigkeit definiert, Teil eines künstlerischen Prozesses zu sein und diesen zu realisieren. Lubart nähert sich dieser Frage in seiner Psychologie der Kreativität und hinterfragt: „Was definiert die Kreativität eines Menschen?“ (Lubart, Mouchiroud, Tordjman \& Zenasni, 2015) Aus dieser Perspektive betrachten wir derzeit unser Modell der Kreativität, ein Multivariaten-Modell, 
das sich aus vier Parametern (conatif, cognitif, émotionnel und environemental, also Motivation, Kognition, Emotion und Umfeld) zusammensetzt.

Sich mitzuteilen ist Grundrecht und Bedürfnis aller Menschen, aller Schüler*innen mit ihren individuellen Möglichkeiten und mit ihrer eigenen Vielfalt. Durch kunstgeleitete, polyästhetische Interaktionen (Bewegung, Tanz, Rhythmus, Sprache, Musik, Malen) können Kommunikation gefördert, Brücken gebaut und Barrieren abgebaut werden.

Denk-Freiräume entstehen in diesem Projekt durch ein Miteinander Sein und gemeinsame zweckgebundene Überlegungen fern von Ausgrenzung. Sinnstiftendes Handeln und Agieren der Schüler*innen in zukunftsweisende Bahnen zu lenken ist Credo unserer Arbeit. Im klassenübergreifenden, gemeinsamen künstlerischen Handeln lernen die beteiligten Schüler*innen ihre Möglichkeiten und Potenziale kennen und diese bewusst einzusetzen. Eine mit und für Schüler*innen entwickelte künstlerische Rahmung für die Gestaltung von inklusiven Räumen, die es ermöglicht, Raum über Grenzen von individuellen Charakteristika, Klassifikationen und Zuschreibungen als durch und durch inklusiv zu erleben.

Lehrpersonen, Eltern-Expert $\left({ }^{*}\right.$ inn)en, Selbstvertreter ${ }^{*}$ innen und Akteurinnen und Akteure der Lehrer $\left({ }^{*}\right.$ innen)bildung werden in den Entwicklungsprozess involviert und so kann über den schulischen Kontext hinaus Inklusion in einem gesamtgesellschaftlichen Verständnis weiterverbreitet werden.

Die der Methode zugrunde liegenden polyästhetischen Interventionen zur Umsetzung von Inklusion läßt dem Individuum genügend Spiel-Raum, einen Raum - im Sinne von Winnicott (2003, S. 37) - der kreativen Aktivität, zu einem unbedingten selbstverständlichen Miteinander-Sein und MiteinanderLeben zu finden.

\section{Von Kunst und Behinderung zu Polyästhetik und Inklusion}

Ausgehend von einer Projektidee (,The Art of Inclusion: Polyästhetische Ansätze in der Inklusion') sollen gemeinsam mit den Schüler( ${ }^{*}$ inne)n inklusive Räume kreiert werden, sodass die räumlich-äußeren Grenzen der Schule von den Schüler( ${ }^{*}$ inne)n überschritten werden können und sich Erfolge wie beispielsweise im sozialen Raum und Umfeld der Schüler*innen einstellen. So könnte ein Kreativraum als Raum zum Austauschen und Entdecken von Möglichkeiten fernab jeglicher Etikettierung und Vereinheitlichung entstehen.

Die grundlegende Vision dieses Projekts hängt mit folgenden Fragestellungen zusammen: 
- Welche Räume werden durch die polyästhetische Methode geöffnet?

- Welche inklusiven Räume werden kreiert, so dass die Ideen von Raum über die Grenzen der Schule hinaus wirken?

- Wie können Schüler*innen einen Kreativ-Raum als Ort des Austausches nutzen? Ist es hier möglich, gemeinsam zu reflektieren, anderen zuzuhören, $\mathrm{zu}$ argumentieren und sich Gedanken zu machen und zu lernen, sich sprachlich auszudrücken über künstlerische Prozesse, die gemeinsam erlebt werden?

Das gemalte Bild wird spontan zu Tanz, der Tanz wird musikalisch interpretiert. Es kommt zu einem permanenten Wechselspiel der Parameter Zeit, Kraft, Raum und Form, welche die Musik, den Tanz und das gemalte Bild gleichermaßen beflügeln. Diese Methode erschließt sich in der spontanen Improvisation und durch die Möglichkeiten, in wacher Bereitschaft mit allen Sinnen Resonanz in sich selbst und im Raum mit anderen zu erleben (vgl. Schnabl, 2016, S. 128). In diesem Setting kommt es zu einem Ein- und Zulassen, zu einem Weitertragen, -bewegen, -tanzen, -malen und -musizieren. Hier kann der tanzende Körper seiner eigenen Gegenwart entfliehen, sich zergliedern und desartikulieren. So löst er sich unmerklich aus sich selbst heraus. Und so lernen wir von Beginn an und zu allermeist durch unsere erlebten körperlichen Erfahrungen (vgl. Hille, 2016, S. 93). Hieraus kann eine neue Sprache entstehen, die so verändert ist, dass sie hinter jede Sprache zurückfällt. „...Und unmerklich geschieht diesem tanzenden Körper das: Er ist nicht länger ein Körper in sich. Er nimmt Spielraum ein. Er nimmt Abstand. Er beginnt sich zu denken. Er tanzt sich, er wird von einem anderen getanzt." (Nancy, 2010, S. 32-33)

Durch diese oben beschriebene Art der kunstgeleiteten Bewegungssprache lernen die Schüler*innen zu abstrahieren und eine bestimmte Form der Interpretation anzuwenden. Sie entfernen sich von einer gewissenhaften 1:1-Erzählung und -Darstellung im Tanz und zeigen mehr Bewusstsein für Wahrnehmung im Raum in ihrem unmittelbaren sozialen Handlungsumfeld. Sie verkörpern diese Erfahrung und können sie wieder abrufen.

\section{Die Rahmung des Projekts}

Im Folgenden geben wir einen Einblick in die Anwendung eines polyästhetischen Modules, welches als Workshop-Konzept mit den Mitteln Tanz, Musik und Bildsprache operiert (vgl. Schnabl-Andritsch, Roy \& Proyer, 2018):

In einem Kick-Off-Workshop laden wir die Schüler*innen ein, durch spezifische Anleitungen technisches und künstlerisches Wissen im zeitgenössischen Tanz, der Musik und im bildnerischen Bereich zu erwerben. In allen ästhe- 
tischen Bereichen ist uns wichtig, den Schüler(*inne)n Werkzeuge zu geben, die ihnen helfen, ihren eigenen künstlerischen Zugang in der jeweiligen Ästhetik zu finden und Aneignung des Raumes kreativ und autonom entwickeln zu können. Über konkrete Angaben zu improvisatorischen Methoden und über das Erleben bestimmter Aktionen im Raum - allein, zu zweit und/oder in der Gruppe - finden sie zu einer sinnlichen Vertiefung der eigenen kreativen Qualität.

Der Workshop beginnt mit einem Begrüßungsritual, bei dem wir uns mit einem Klang- und Rhythmusspiel im großen Kreis zusammenfinden. Danach schickt jede und jeder Teilnehmende eine Geste als Begrüßung in die Gruppe. Diese Gesten werden unisono wiederholt. Die künstlerischen Arbeiten eines vorangegangenen Workshops werden zur Brücke zu raumbezogenen Aufträgen oder zu Übungen der aktuellen Einheit. Ein Beispiel dafür ist die Raumpartitur (siehe Abb. 3). Hierbei handelt es sich um eine Übung, bei der die Schüler*innen gemeinsam entscheiden, an welchen Punkten im Raum sie ihre gemeinsame Aktion beginnen und wo diese endet. Dabei stehen ihnen musikalische, tänzerische und bildhafte Mittel zur Auswahl, um zu einer von ihnen selbst gewählten Raumdefinition (z.B. Freiheit, Gemeinschaft, Freundschaft) zu kommen. In der nachfolgenden Abbildung ist eine bildhafte Dokumentation dargestellt.

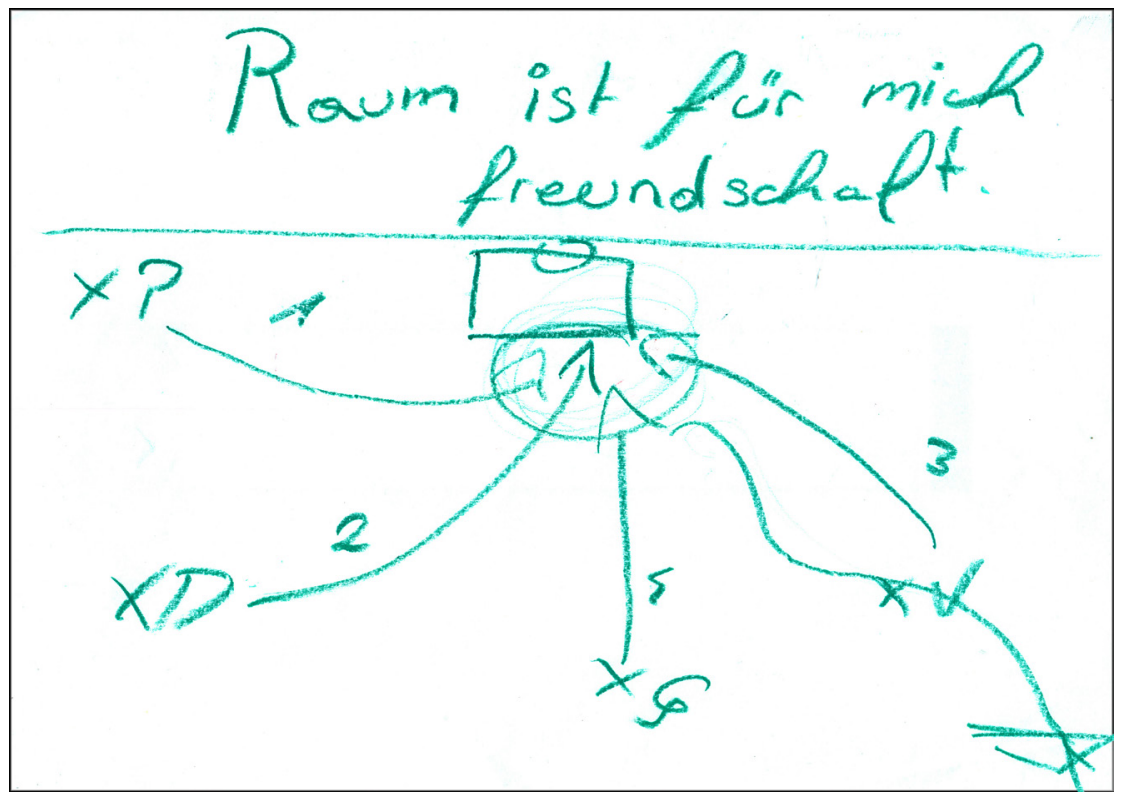

Abbildung 3: Beispiel einer Raumpartitur, von Schüler( ${ }^{*}$ inne)n gefertigt 
In der Komplexität der folgenden Aufgabe übertragen die Schüler*innen ein bildnerisches Objekt (z. B. ein gemaltes und/oder beschriftetes Bild) in eine räumliche Dimension: In kleinen Teams (drei bis vier Schüler*innen) improvisieren sie tänzerisch und rhythmisch-musikalisch und fertigen eine Raum-Partitur an. Sie vertiefen im Vorzeigen ihre Bewegungen während andere Schüler*innen das kompositorische Geschehen wahrnehmen und analysieren. Jede Idee jedes Teammitgliedes wird gehört und auf ihre Umsetzbarkeit hin kritisch überprüft. Jedes Team hat genügend Zeit, eine Raum-Partitur in Bewegung zu entwerfen, auszuprobieren und zu festigen. Diese Raumpartituren (siehe Abb. 3) werden in der großen Runde präsentiert.

In der hier beschriebenen Aufgabe wird der Fokus des räumlich-tänzerischen Bereiches deutlich, wo die Zeichnung die Zweidimensionalität verlässt zu Gunsten der tänzerisch-räumlichen Partitur.

Am Ende des Workshops werden die Kinder gebeten alles im Workshop Erlebte zu dokumentieren. Dazu dient eine gemeinsame transparente Plastikplatte. Das Erlebte wird anhand von im Organisationsteam entwickelten Leitfragen zur Evaluierung des Workshop-Geschehens diskutiert (z. B. „Wie habt ihr den Raum heute erlebt?").

\section{Fazit}

Bei diesem Workshop werden Klassenzimmer und Schulraum als Nucleus einer inklusiven Gesellschaft verstanden und es wird davon ausgegangen, dass kunstgeleitete Methoden inklusives Lernen ermöglichen. Ein Lernen mithilfe solcher Methoden kann spezifische Erfahrungs- und Entwicklungsräume eröffnen und ein positives Verständnis von Vielfalt, von unterschiedlichen $\mathrm{Zu}$ gängen und von mehrperspektivischen Betrachtungsweisen fördern. Durch eine angewandte Polyästhetik im Schulalltag (über tänzerisch-musikalisches und bildnerisch-gestaltetes Handeln) sollen Schüler*innen eine Neuorientierung ihres Handlungsspielraumes erleben.

Schüler ${ }^{*}$ innen und Lehrer*innen tragen gemeinsam mit Wissenschaftler ${ }^{*}$ inne $) n$, Tänzer $\left({ }^{*}\right.$ inne $) n$ und Musiker $\left({ }^{*}\right.$ inne)n praktische Anleitungen nach außen in ihr soziales Umfeld, um Pädagog $\left({ }^{*}\right.$ inn $)$ en, Eltern und interessierten Menschen Bausteine in die Hand zu geben, mit denen sie in der Praxis arbeiten können. Die Form des aus dem Workshop hervorgehenden Konzepts, das die Anwendung in jeglichem Bildungskontext ermöglicht, wurde gemeinsam mit den Schüler(*inne)n erarbeitet. Ziel ist nicht, ein fertiges, abgeschlossenes Endprodukt (z.B. eine einmalige leistungsorientierte Performance) $\mathrm{zu}$ erstellen, sondern durch polyästhetische Methoden den Teilnehmer ${ }^{*}$ inne)n Autonomie in ihrer Kreativität zu ermöglichen, ferner durch den krea- 
tiven Diskurs, der durch die Polyästhetik entsteht, Freiheitsräume und DenkFreiräume zu initiieren, so dass sich die Schüler*innen auch außerhalb der Schule damit getragen fühlen.

\section{Literatur}

Baier, F. X. (2000). Der Raum. Köln: Verlag der Buchhandlung Walter König. Berufsverband der TanztherapeutInnen Deutschlands (BTD) (2020). Ethikkodex für Tanztherapie. Verfügbar unter: https://www.btd-tanztherapie.de/uploads/ File/2_Ethikkodex.pdf [17.06.2020].

Brun, A., Chouvier, B. \& Roussillon, R. (2013). Manuel des médiations thérapeutiques. Paris: Dunod.

Ehlers, M. (2007). Grenzwahrnehmung. Poetiken des Übergangs in der Literatur des 19. Jahrhunderts. Kleist-Stifter-Poe. Bielefeld: transcript. https://doi.org/ $10.14361 / 9783839407608$

Eisenhauer, J. (2007). Just looking and staring back: Challenging ableism through disability performance art. Studies in Art Education, 49 (1), 7-22. https://doi.org/ 10.1080/00393541.2007.11518721

Gutjahr, E. (2014). Improvisation und Kreativität In M. Steffen-Wittek \& M. Dartsch (Hrsg.), Improvisation (S. 20-30). Regensburg: ConBrio.

Hegi, F. (2005). Das Klangraumgefühl. Musiktherapeutische Umschau, 26 (3), 72-77. Heule, R. (2009). Auf dem Weg in die Schule-mit Orientierung geht es besser. In Arbeitsgemeinschaft Frühförderung sehgeschädigter Kinder (Hrsg.). Verfügbar unter http://www.bewegung-im-dialog.de/sites/default/files/literatur/artikelfas sung_auf_dem_weg_in_die_schule_workshop_ag_ff_5-09.pdf [17.06.2020].

Hille, P. (2016). Emile Jaques-Dalcroze und die Erziehung durch Musik und Bewegung. In A. Hauser-Dellefant \& E. Witoszynskyj (Hrsg.), Leben ist Bewegung ist Musik (S. 93). Wiesbaden: Reichert Verlag.

Lubart, T., Mouchiroud, C., Tordjman S. \& Zenasni F. (2015). Psychologie de la créativité. Paris: Armand Colin.

Menzen, K.-H. (2009). Grundlagen der Kunsttherapie. München: Ernst Reinhardt Verlag.

Nancy, J. L. (2010). Ausdehnung der Seele. Zürich: Diaphanes.

Nind, M. (2014). What is inclusive research? London, New Delhi, New York, Sydney: Bloomsbury.

Schnabl, N. (2016). Improvisation in Musik und Bewegung. In A. Hauser-Dellefant \& E. Witoszynskyj (Hrsg.), Leben ist Bewegung ist Musik (S. 123-128), Wiesbaden: Reichert Verlag.

Schnabl-Andritsch, N., Roy, V. \& Proyer, M. (2018). Angewandte Polyästhetik in der Kunst der Inklusion, In S. Quinten \& C. Rosenberg (Hrsg.), Tanz-Diversität-Inklusion (S. 205-218). Bielefeld: transcript. https://doi.org/10.14361/97838 39443309-016

Winnicott, D. W. (1971, S. 37 \& engl. 2003). Jeu et réalité. Paris: Gallimard. 


\section{Capoeira als inklusive Praxis}

\section{Pädagogisches Potenzial einer brasilianischen Widerstandsbewegung}

\author{
Capoeira veio daqui \\ do quilombo de Zumbi \\ como Angola ela chegou \\ e aqui na luta formou \\ Negro fugia da senzala \\ perseguido \\ e escondia do alarido \\ pra lutar \\ olha armada, meia lua, \\ cabeçada \\ a rasteria e a queixada \\ pra matar \\ capoeira dizia \\ capoeira fazia \\ liberdade pro negro \\ liberdade pra vida \\ capoeira hoje em dia \\ é a vida alegria \\ vem pra roda vamos jogar
}

\author{
Capoeira kam von hier \\ aus dem quilombo von Zumbi \\ als Angola kam sie her \\ und hier formte sie den Kampf \\ Die Neger ${ }^{1}$ flohen aus den Sklavenhütten \\ stets verfolgt \\ und versteckten sich vor dem Geschrei \\ bereit zu kämpfen \\ schau die Armada, die Meia Lua, \\ die Cabeçada \\ die Rasteria und die Queixada ${ }^{2}$ \\ bereit zu töten \\ der Capoeirista sprach \\ der Capoeirista handelte \\ Freiheit für die Neger \\ Freiheit für das Leben \\ Capoeira heutzutage \\ ist eine Lebensfreude \\ komm zur roda, lass uns spielen
}

Lied der Capoeira (aus Hegmanns, 1998, S. 80)

\section{Einleitung}

Capoeira ist die harmonische Verbindung von Kampf, Tanz und Musik. Wie das eingangs zitierte Lied der Capoeira (Hegmanns, 1998, S. 80) deutlich macht, ist Capoeira als brasilianischer Kampftanz Sinnbild des Widerstands, der Lebensfreude und der „Freiheit für das Leben“ (Hegmanns, 1998, S. 80) der aus Afrika nach Brasilien verschleppten Sklavinnen und Sklaven. Als sol-

1 Dirk Hegmanns, aus dessen Buch Capoeira - Die Kultur des Widerstandes dieses Lied der Capoeira stammt, schreibt zu dem Begriff Neger in diesem Zusammenhang: „In vielen Liedern kommt ein starkes Selbstbewusstsein der Farbigen zum Ausdruck und sie bezeichnen sich bewusst als ,Neger“ (negro, negó oder negão). Sie benutzen diesen Begriff als Selbstbekenntnis zu ihrer Hautfarbe und kehren so seinen üblichen Gebrauch als Beleidigung ins Gegenteil: Die schwarze Hautfarbe ist ihr Stolz." (Hegmanns, 1998, S. 62)

2 Armada, Meia Lua, Cabeçada, Rasteria und Queixada bezeichnen Techniken der Capoeira. 
che erfährt Capoeira auch zunehmend internationale Beachtung. Der vorliegende Artikel versucht zu erörtern, inwiefern diese Form der musikalisch-tänzerischen Betätigung richtungsweisend für eine inklusive pädagogische Praxis sein kann. Dabei wird zunächst die historische Entwicklung der Capoeira dargestellt, um daraus deutlich den ihr zugrunde liegenden Widerstand gegen Exklusion - den inklusiven Kern der Capoeira - hervorzuheben. Anschließend wird ausgeführt, was dieser inklusive Kern in der Praxis bedeutet und warum sich Capoeira besonders eignet, um Menschen mit den unterschiedlichsten sozialen, körperlichen und geistigen Voraussetzungen zusammenzubringen.

\section{Grundlagen der Capoeira}

Als die drei Grundelemente der Capoeira gelten der Kampf, der auch als Spiel oder Tanz bezeichnet werden kann, die Musik und die Verbindung dieser beiden Elemente in der roda (dem Kreis), der Musik und Kampftanz miteinander vereint. Während der Mestre, die Lehrperson, gemeinsam mit einigen anderen Capoeiristas an verschiedenen Instrumenten, wie Caxixis, Trommeln, Schellenkränzen und vor allem dem Hauptinstrument, dem Berimbau, ein Lied anstimmt, welches in seiner Form einem Frage- und Antwortspiel gleicht, stehen die anderen Teilnehmer*innen der roda im Kreis, klatschen im Rhythmus und antworten singend auf den Gesang des Mestres. Zwei Capoeiristas treten hervor, hocken sich im Kreis einander gegenüber, klatschen sich mit den Händen $\mathrm{ab}$ und eröffnen das Spiel mit einem gemeinsamen Radschlag. Danach entfaltet sich im Spiel mit den Körpern ein Frage- und Antwortspiel zwischen Angriffs- und Abwehrbewegungen. Nach und nach werden die beiden Kämpfer*innen oder Tänzer*innen von anderen Capoeiristas aus dem Kreis abgelöst, das Spiel geht weiter und kann bis zu zwei Stunden dauern.

Das besondere Merkmal, das die Capoeira von eigentlichen Kampfsportarten grundlegend unterscheidet, ist die Tatsache, dass es hierbei keine Berührung zwischen den Akteur $\left({ }^{*}\right.$ inn)en im Kreis gibt. So bleibt es beim Tanz, bei einem Spiel aus Aktion und Reaktion. Neben dem Erlernen der Bewegungen, die in hohem Maße die Körperbeherrschung fördern, ist auch ein Unterricht an den Musikinstrumenten und die Vermittlung von Philosophie und Geschichte der Capoeira ein integraler Bestandteil der Ausbildung (vgl. Hegmanns, 1998, S. 14). Capoeira ist somit nicht nur ein Sport, sondern eine Lebenseinstellung und Philosophie, eine „Kultur des Widerstandes“ (Hegmanns, 1998). Dabei sollte Widerstand, so Röhring Assunção, weniger im Sinne einer heroischen Haltung und offener Konfrontation verstanden werden, als im Sin- 
ne einer „flexiblen Aneignung und subtilen Selbstbehauptung“ (Röhrig Assunção, 1999, S. 1) $)^{3}$.

\section{Capoeira - eine Geschichte von Exklusion und Inklusion}

Die Ursprünge dieser Widerstandsphilosophie reichen nach Hegmanns (1998) weit in die Geschichte Brasiliens zurück. Bereits kurz nach der europäischen Entdeckung Brasiliens durch Pedro Àlvares Cabral (1500) wurden ab dem Jahr 1538 Millionen von Afrikaner( ${ }^{*}$ inne)n als Sklav(*inn)en nach Brasilien verschleppt. Diese Verschleppungen und die Ausbeutung der Versklavten hielten bis 1888 an, dem Jahr, in dem Brasilien als letztes Land auf dem amerikanischen Kontinent die Sklaverei offiziell abschaffte. Die Schätzungen zur Anzahl der insgesamt verschleppten Menschen in den 350 Jahren der Sklaverei schwanken zwischen 2,3 und 18 Millionen. Eine genaue Rekonstruktion des tatsächlichen Ausmaßes der Verschleppungen wurde unmöglich, nachdem im Jahr 1888 Ruy Barbosa, der damalige Landwirtschaftsminister, alle Unterlagen hierzu vernichten ließ (vgl. Hegmanns, 1998, S. 15).

Die Versklavung der Menschen aus Afrika, ihre Ausbeutung und Unterdrückung, wurde von Beginn an systematisch durch die Zerschlagung ihrer Traditionen und Gemeinschaften vollzogen: So wurden afrikanische Familien und Stammesgruppen bereits auf der Überfahrt nach Brasilien bewusst auseinandergerissen, um den Zusammenhalt und ihre gemeinsame Identität zu zerstören. Auf diese Weise sollten auch mögliche Aufstände von vornherein unterbunden werden. Nur in der Religion konnten sich auch über die Jahrhunderte der Unterdrückung hinweg einige Traditionen erhalten, die nicht durch die Kolonialist(*inn)en zerstört wurden (vgl. Höllinger, 2007, S. 53).

Die Gewährung religiöser Freiheiten wird zum einen auf einen generellen Reiz des Unbekannten zurückgeführt, den die Kolonialist(*inn)en beim Anblick der afrikanischen Riten verspürt haben sollen, zum anderen auf ihre Faszination für die heilende oder schädigende Wirkung der sogenannten Wundermittel ${ }^{4}$ aus den afrikanischen Traditionen (vgl. Hegmanns, 1998, S. 17-18). Darüber hinaus war eine flächendeckende Konvertierung der Versklavten zum

3 Interessante Ausführungen zum zwiespältigen Verhältnis der Capoeira zwischen Anpassung und Widerstand finden sich in Röhrig Assunção (1999).

$4 \mathrm{Zu}$ diesen Wundermitteln ist unter anderem die in den afrobrasilianischen Religionen Camdomblé und Umbanda gängige Praxis der Trance zu verstehen: „Der Mensch wird ein ,anderer, d.h. sie oder er besitzt die Macht, viele andere Persönlichkeiten zu sein, die nicht mit der Alltagsidentität identisch sind. Obwohl der Körper, die Gesichtszüge, die ethnische Herkunft dieselbe bleiben, verhält sich der Mensch durch das Eindringen des Geistes mit andersartigen Charakterzügen in einer nicht wiedererkennbaren Art und Weise. Frauen sprechen plötzlich mit der 
Katholizismus aufgrund sprachlicher Hürden schwierig und so galt die Religion und die Ausübung religiöser Praktiken als ein erwünschtes trennendes Merkmal zwischen Herrschenden und Versklavten, welches zusätzlich die Hierarchien verdeutlichen sollte. Die religiösen Riten der Versklavten waren dabei verbunden mit Musik und Tanz und so waren ihre Besitzer*innen an das Bild der tanzenden und musizierenden Sklavinnen und Sklaven gewöhnt und schenkten den Ritualen kaum Beachtung (vgl. Hegmanns, 1998, S. 17-18).

Ende des 16. Jahrhunderts konnten sich „einige Dutzend afrikanischer Sklaven“ (Hegmanns, 1998, S. 8) aus der Unterdrückung durch ihre Herrschenden befreien und flohen von den Zuckerrohrfeldern in die Wälder von Palmares im Nordosten Brasiliens (vgl. Hegmanns, 1998, S. 8; Onori, 1988, S. 14-15). Hier gründeten sie, gemeinsam mit anderen an den Rand der Gesellschaft gedrängten Menschen (vgl. Onori, 1988, S. 15), die Quilombos, versteckte Siedlungen, in denen sie weitgehend autark lebten und wo sich eine Philosophie des Widerstandes - initiiert durch die eigenständige Befreiung aus der Unterdrückung der weißen Kolonialist( ${ }^{*}$ inn)en - im jahrzehntewährenden Widerstand entwickeln konnte. Für die ehemalig Versklavten stellte somit ein Raum der Exklusion die einzige Möglichkeit dar, um frei und selbstbestimmt leben zu können.

$\mathrm{Zu}$ dieser Zeit, mit dem Beginn einer Widerstandsbewegung gegen die Unterdrückung und Versklavung der farbigen Bevölkerung Brasiliens, breitete sich unter den Versklavten - sowohl unter denen, die in Gefangenschaft lebten, als auch unter den Befreiten in den Quilombos - eine Form des Kampfes aus, „deren Entstehung eng verknüpft ist mit dem Streben der Schwarzen nach Freiheit: die Capoeira" (Hegmanns, 1998, S. 9). Woher diese Art zu kämpfen ursprünglich kam, ist historisch nicht eindeutig belegt. Theorien dazu beruhen eher auf überlieferten Mythen und Sagen. So könnte Capoeira einerseits als ein Tanz des Krieges zu verstehen sein, zurückzuführen auf eine „Mischung unterschiedlicher Kampfstile, Tänze, Rituale und Instrumente aus verschiedenen Teilen Afrikas. Eine Mischung, die auf brasilianischem Boden verwirklicht wurde" (Capoeira, 2008, S. 30). Auch besteht die Möglichkeit, dass die Capoeira nicht nur in ihren einzelnen Bestandteilen von Kampf, Tanz, Ritual und Musik, sondern als eigenständiges Element bereits aus Afrika stammt und mit den verschleppten Sklav( ${ }^{*}$ inn)en nach Brasilien kam. Eine weitere Theorie sieht die Entstehung der Capoeira hingegen in einer Kampfkunst, „die sich als Tanz tarnte, um der Verfolgung durch die Verwalter und Besitzer der Zuckerrohrfabriken zu entgehen“ (Capoeira, 2008, S. 32). Demnach hätte sich die Capoeira aus einem brutalen Kampfspiel entwickelt und sich durch die Unter-

Stimme eines Mannes, Junge bewegen sich wie Greise und umgekehrt.“ (Scharf da Silva, 2004, S. 136) 
drückung zu dem weiterentwickelt, was wir heute gemeinhin unter Capoeira verstehen.

Unabhängig davon, woher die Capoeira stammt, breitete sie sich sicherlich nicht zufällig in einer Zeit des aufkeimenden Widerstandes aus. Die Capoeira gab den Versklavten die Möglichkeit, sich selbst als wirkmächtig zu erleben, Kräfte zu bündeln und zu steuern, widerständig zu sein und dem inneren Streben nach Freiheit Ausdruck zu verleihen. Und nicht zuletzt gab die Capoeira der farbigen Bevölkerung ein Gefühl der Gemeinschaft - ein Wir, das gehört und ernst genommen werden wollte.

Die Zeit der Quilombos, in der sich die Philosophie des Widerstandes entwickeln konnte, dauerte beinahe hundert Jahre. Jedoch konnte auch die Ausbreitung der Capoeira - als kämpferischer Ausdruck dieser Widerstandsphilosophie - nicht verhindern, dass die Quilombos „durch die Kanonen der Portugiesen dem Erdboden gleichgemacht und fast sämtliche in ihnen lebenden Menschen niedergemetzelt wurden“ (Hegmanns, 1998, S. 9).

„Als im Sommer des Jahres 1695 die Soldaten der portugiesischen Armee in die dichten Wälder von Gongoro drangen und sich ihnen Zumbi, der große König der Quilombos von Palmares, ein letztes Mal entgegenstellte und den Tod der Kapitulation vorzog, ging eine Epoche brasilianischer Geschichte zu Ende, die in dem Land und in seiner Kultur bis heute ihre unverwechselbaren Spuren hinterlassen hat. Denn Zumbi lebt weiter, lebt in den Herzen der farbigen Bevölkerung Brasiliens, lebt in Liedern und Tänzen und lebt in den Geschichten - wahren und erfundenen - die vom hundert Jahre langen

Kampf der schwarzen Sklaven gegen die portugiesischen und holländischen Kolonialherren Brasiliens erzählen."

(Hegmanns, 1998, S. 8)

Mit der von Hegmanns beschriebenen Niederschlagung der Quilombos und der Mystifizierung Zumbis (vgl. Hegmanns, 1998, S. 8) wurde die Capoeira von Seiten der weißen Kolonialist( ${ }^{*}$ inn)en unterdrückt und verboten. Capoeiristas, die man beim Kampf ertappte, wurden massiv bestraft, mit Maßnahmen, die teilweise zum Tod führten (vgl. Capoeira, 2008, S. 32). Die Verfolgung der Capoeiristas steigerte sich noch, als König D. João VI. von Portugal 1808 ins Exil nach Brasilien flüchtete. Dort sah er die Notwendigkeit, die Kultur eines Volkes zu zerstören, um es zu erobern - die Capoeira und die ganze Kultur der schwarzen Bevölkerung wurden unterdrückt und die Ausübung der Capoeira schließlich 1890 per Gesetz im ersten Strafgesetzbuch der Republik verboten (vgl. Capoeira, 2008, S. 30). Von da an konnte die Capoeira 
nur noch im Untergrund überleben. In dieser Zeit entwickelten sich wohl auch die typischen Formen des Kampftanzes, wie sie heute noch praktiziert werden: „Das Spiel am Boden und im Stand, einige akrobatische Bewegungen wie das Rad, die Benutzung des Berimbau", der Kreis, das Ritual etc." (Capoeira, 2008, S. 39) Erst in den 1930er Jahren wurde die Capoeira unter Präsident Getúlio Vargas unter Auflagen wieder erlaubt ${ }^{6}$. Die Eröffnung der ersten Akademie für Capoeira in Salvador durch Mestre Bimba - mit bürgerlichem Namen Manoel dos Reis Machado - legte den Grundstein für die Anerkennung der Capoeira auch in der bürgerlichen Mittelschicht und auch für eine Ausbreitung der Akademien (den Ausbildungszentren der Capoeira) über die Grenzen Brasiliens hinweg.

Es ist also heute nicht mehr (oder noch nicht) eindeutig nachvollziehbar, wann, warum und wo die Capoeira entstanden ist. Klar aber ist, dass sie in dieser Form, angesiedelt zwischen Kampf und Tanz - immer verbunden mit der ihr eigenen Musik -, bis heute weiterlebt und international immer mehr Interesse weckt. Capoeira war schon immer eine Form des Widerstandes, dient und diente der Selbstbefähigung und kann als Auflehnung gegen Exklusion verstanden werden: „Capoeira ist also untrennbar verbunden mit der Geschichte der Farbigen in Brasilien und mit ihrer jahrhundertelangen Auflehnung gegen Ungerechtigkeiten und Diskriminierung. Somit ist sie eine politische Sportart, die ohne ein Bewusstsein über die historischen und sozialen Hintergründe nicht praktiziert werden kann.“ (Hegmanns, 1998, S. 12)

Während Capoeira aus dieser Tradition heraus unabdingbar mit der farbigen Bevölkerung verbunden war, strömen heute immer mehr weiße Brasilianer der Mittel- und Oberschicht in die brasilianischen Capoeira-Akademien und lassen die Grenzen zwischen Rassen, Schichtzugehörigkeiten und anderen Diversitätsmerkmalen verschwimmen (vgl. Hegmanns, 1998, S. 14). Darüber hinaus kommt gerade der Capoeira heute eine hohe Bedeutsamkeit in der Arbeit mit Straßenkindern und Kindern in Armenvierteln zu, um sie in Gemeinschaften einzubinden und sie anhand der historischen Widerstandsphilosophie, deren Vermittlung immer wesentlicher Bestandteil der Ausbildung ist, $\mathrm{zu}$ motivieren und zu befähigen, sich selbstbewusst ihren von außen gesetzten Grenzen zu stellen.

5 Der Berimbau ist das typische Instrument der Capoeiristas: Ein Musikbogen bestehend aus einem gebogenen Holzstock, einem an beiden Enden befestigten Draht als Saite und einem aufgeschnittenen, ausgehöhlten Flaschenkürbis, der als Resonanzkörper dient.

6 So war zunächst nur eine überwachte Ausübung der Capoeira in geschlossenen Räumen und nur mit polizeilicher Genehmigung erlaubt (vgl. Capoeira, 2008, S. 42). 


\section{Capoeira als Gemeinschaft ohne Grenzen}

Die Geschichte der Capoeira macht gerade auch im Hinblick auf ihre zunehmende Ausbreitung und Anerkennung weltweit deutlich, dass sie vom Rand der Gesellschaft kommt und heute Teil der Gesellschaft ist. Schon die Geschichte der Capoeira kann somit als eine Geschichte von Exklusion und Inklusion verstanden werden. Aber auch die Praxis der Capoeira - das Spiel, der (Kampf-)Tanz und die Musik - weist besondere Qualitäten für ein inklusives Tanz- und Musikerleben auf:

1. Jede ${ }^{\star} r$ kann nach ihrenseinen Fähigkeiten teilnehmen. Ein ${ }^{\star} e$ Anfänger ${ }^{\star}$ in kann genauso mit jemand Fortgeschrittenem agieren, wie die Mestres untereinander. Wichtig ist immer, - egal auf welchem Niveau - sich aufeinander abzustimmen und miteinander ins Spiel zu kommen. So ist es auch möglich, dass Menschen mit schweren körperlichen Beeinträchtigungen oder anderen Behinderungen an der roda teilnehmen, dort eine Bühne finden und ihr individuelles Spiel entfalten können.

2. Das Gemeinschaftserleben ist ein wichtiges Merkmal der Capoeira. Auch Gesang und Musik sind ein wesentlicher Teil der roda und bieten somit für Jede und Jeden die Möglichkeit, Teil des Geschehens zu sein, auch wenn sie oder er (gerade) nicht am eigentlichen Spiel im Kreis teilnehmen kann oder möchte.

3. Auch in der Capoeira gibt es Leistungskriterien und Bewertungen. Ähnlich wie in anderen Kampfsportarten können nach Alter und Können unterschiedliche Gürtel, cordas, erworben werden. Diese Leistungsdifferenzierungen schließen jedoch niemanden vom Spiel aus.

Es wird deutlich, dass bei der Capoeira zu keiner Zeit jemand ausgeschlossen ist. Musik und Tanz werden auf dem je individuellen Leistungsniveau praktiziert und trainiert und zugleich treffen in der roda, dem eigentlichen Spiel der ganzen Gemeinschaft, alle Niveaus aufeinander und können miteinander interagieren. Trotz einer möglichen Leistungsdifferenzierung sind alle Teil des Geschehens.

Die Widerstandsphilosophie der Capoeira kann darüber hinaus gerade auch diejenigen Menschen ansprechen, die sich selbst aus der Gesellschaft ausgeschlossen oder aber in der Gesellschaft behindert fühlen. So können die Tradition des Widerstandes, aber auch das ganz praktische Erleben des Vorrückens vom Rand des Geschehens in die Mitte einer Gruppe und damit in den Fokus der Aufmerksamkeit, einen Prozess in Gang setzen, der jeden Menschen dazu ermutigt, sich gegen die ihm gesetzten Grenzen aufzulehnen und seinen Platz in der Gesellschaft einzufordern. An diesem Punkt setzt auch eine Pä- 
dagogik an, die Hoffmann (2018) in Anlehnung an die lateinamerikanische Theologie der Befreiung und vor allem die Pädagogik der Unterdrückten von Paulo Freire (1973) als Pädagogik der Befreiung beschreibt: Im Mittelpunkt einer solchen Pädagogik der Befreiung „steht [...] die konkrete Bestimmung der menschlichen Möglichkeiten und ihrer Unterdrückung. Sie muss über die reine Anerkennung von Gleichheit und Differenz hinausweisen, da niemand weiß, was der Mensch außerhalb von Herrschaft, Gewalt, Unfreiheit oder Unterdrückung sein kann.“ (Hoffmann, 2018, S. 39)

Während die Capoeira inzwischen auch international zunehmend Aufmerksamkeit erfährt ${ }^{7}$ und sich auch im europäischen Raum die Capoeira-Akademien immer weiter ausbreiten und etablieren, ist die wissenschaftliche Auseinandersetzung gerade auch im Hinblick auf den sozialen Wert der Capoeira hierzulande noch nicht angekommen. In der brasilianischen Literatur finden sich bereits Arbeiten, die sich mit dem pädagogischen und psychosozialen Potenzial der Capoeira, vor allem im Hinblick auf soziale Inklusion, befassen (u. a. Ferreira, 2012; Palhares, 2007). So führt zum Beispiel Tarcísio José Ferreira aus, dass es ein wesentlicher Vorteil der Capoeira sei, dass sie in verschiedenen sozialen Gruppen praktiziert werden könne: „Der Einsatz der Capoeira als Werkzeug für die soziale Entwicklung ist sehr vielseitig, bringt sie das Individuum doch dazu, einen Platz in einer sozialen Gruppe einzunehmen und sich dessen Bedeutung und der Wirkung in der Gruppe bewusst zu sein“ (Ferreira, 2012, S. 43; Übersetzung: M.H.). Auch Leandro Ribeiro Palhares sieht das Potenzial der Capoeira vor allem in ihrem zusammenführenden Charakter, der es jeder und jedem ermöglicht, Teil der Kultur zu sein. Für ihn ist soziale Inklusion „ein Prozess, in welchem das Individuum ein Mitglied der Gesellschaft wird" (Palhares, 2007, S. 12; Übersetzung: M.H.). Bei der Capoeira spiele, so Palhares, selbst in formalen Bildungsprozessen das Gefühl von Freizeit eine wesentliche Rolle, indem sie den Praktizierenden, also den Schülerinnen und Schülern der Capoeira, die Möglichkeit gebe, kreativ zu sein, „zu spielen, zu lachen, zu verdrängen und herumzuhängen" (Palhares, 2007, S. 13; Übersetzung: M.H.). Somit werde die Capoeira zu einem „dynamischen Element der Erzeugung [...] von Kultur. Auf diese Weise hat die Capoeira das Potenzial, soziale Inklusion zu fördern und $\mathrm{zu}$ einer offenen und kritischen Auseinandersetzung mit dem sozialen Zusammenleben beizutragen" (Palhares, 2007, S. 13; Übersetzung: M.H.). Um diesen inklusiven Charakter zu fördern, sei es jedoch wichtig, genügend Raum und Zeit für die Praxis zu bieten, die

7 Die internationale Verbreitung der Capoeira lässt sich besonders gut an den Teilnehmerstaaten bei der Capoeira-Weltmeisterschaft aufzeigen: Bei der WM 2018 in Baku, Aserbaidschan waren insgesamt Teilnehmende aus 46 Nationen gemeldet (vgl. https://azertag.az/en/xeber/World_Championship_on_Sport_Capoeira_starts_ in_Baku-1161930[07.08.2020]). 
Debatte über Geschichte, Kultur und Techniken der Capoeira anzuregen und insbesondere den spielerischen Charakter, die individuelle Kreativität in den Mittelpunkt zu stellen (vgl. Palhares, 2007, S. 13).

\section{Capoeira in der Arbeit mit Schüler( ${ }^{*}$ inne)n mit Verhaltensauffälligkeiten und emotionalen Schwierigkeiten}

„Die Capoeira ist eine Kunst, ein Tanz, eine Philosophie, bei welcher der Capoeirista seine Wünsche und sein Verlangen ausdrückt, etwas, das bei Jugendlichen im Alltag häufig unterdrückt wird und beim Capoeira, inmitten der roda oder auch nur beim Zuschauen, es dem Jugendlichen gelingt, sich von der Welt zu lösen, in eine andere Dimension einzutreten und den Stress des Alltags hinter sich zu lassen." (Ferreira, 2012, S. 32; Übersetzung: M.H.)

Nach Ferreira (2012) bietet Capoeira insbesondere für Jugendliche die Möglichkeit, in der roda ihre Schwierigkeiten und den Stress des Alltags hinter sich zu lassen und einen Zugang zu ihren positiven Emotionen zu bekommen. Besonders interessant scheint die Capoeira somit auch im Hinblick auf die Arbeit mit verhaltensauffälligen oder emotional belasteten Kindern und Jugendlichen zu sein. Auch viele NGOs und sozialpädagogische Institutionen entdecken in den Praktiken der Capoeira einen wesentlichen Kern sozialpädagogischer Arbeit, gerade auch für Kinder und Jugendliche aus schwierigen sozialen Verhältnissen (vgl. Döring, 2010, S. 71). In diesem Bereich entstehen in Brasilien, besonders im Hinblick auf die Arbeit mit Straßenkindern, immer mehr Projekte, um Kinder durch die Capoeira von der Straße zu holen, ihnen eine Tagesstruktur zu bieten, sie an Regeln heranzuführen und das soziale Miteinander zu stärken (u.a. das Projekt Tribo Mirim in São Paulo ${ }^{8}$ ). Persönliche Erfahrungen der Autorin zwischen 2005 und 2012 aus einem Projekt in einem Armenviertel in Bahia zeigen, dass die Capoeira gerade unter den Ärmsten eine respektable Freizeitaktivität darstellt und dass Kinder, die die Philosophie der Capoeira wirklich verinnerlichen und dadurch in der Lage sind, sich besser an Regeln zu halten und empathisch zu agieren, sich engagieren und trainieren, auch in der Schule weiter vorankommen und klarere Lebensziele ent-

8 Das Projekt Tribo Mirim wurde in São Paulo von Ronivon Santos gegründet, um Kinder von der Straße zu holen. Inzwischen expandierte das Projekt nach Mosambik und Angola. Mehr Informationen unter https://www.facebook.com/ projetotribomirim/ [06.08.2020] und im Video unter https://www.youtube.com/ watch?time_continue $=110 \& v=$ s7aWtfFMAt8\&feature=emb_logo [06.08.2020]. 
wickeln. Empirische Untersuchungen zu einer solchen These gibt es bisher nicht, wären jedoch durchaus interessant und wünschenswert.

Gerade in der Arbeit mit Schüler( ${ }^{*}$ inne)n mit aggressiven oder traumatisierten Verhaltensmustern werden asiatische Kampfsportarten wie Karate und vor allem Judo empfohlen (vgl. Theunissen, 2016; Baumann, Koring, Worms \& Aring, 1997). „Aufgrund der kanalisierten körperlichen Auseinandersetzung durch die Strukturen und die besondere Etikette des Judotrainings [...] sind wir der Überzeugung, dass mit Hilfe des Judotrainings [die oder] der Jugendliche lernt und trainiert, sich weniger mit Gewalt durchzusetzen oder körperliche Auseinandersetzungen zu suchen." (Baumann et al., 1997, S. 129). Darüber hinaus könnten auch Schülerinnen und Schüler mit geringem Selbstbewusstsein, ängstlichem oder depressivem Verhalten vom Judotraining profitieren (vgl. Theunissen, 2016, S. 175).

Betrachtet man die Capoeira eher von der Seite des Tanzes, lässt sich für die Arbeit mit der hier beschriebenen Klientel deutlich weniger eindeutige Literatur finden. In Bezug auf Kinder und Jugendliche mit emotionalen Schwierigkeiten schreibt Quinten: „Kreativer Tanzunterricht eignet sich [damit] besonders gut, Selbstvertrauen, Selbstbewusstsein und das Gefühl für Selbstwirksamkeit bei Schülerinnen und Schülern zu stärken." (Quinten, 2016, S. 371) Und auch Behrens und Tiedt kommen zu dem Schluss: „Bewegung und Tanz helfen, den eigenen Körper, seine Potenziale und Grenzen zu spüren, zu nutzen, zu akzeptieren und zu respektieren. Auch dies dient in der sozialen Arbeit oftmals kompensatorischen Zwecken, zumal die Zielpersonen vielfach ein gestörtes Körperverhältnis haben - beispielsweise durch Gewalt- und Missbrauchserfahrungen, durch Unter- oder Übergewicht sowie durch (Sucht-) Krankheiten und Behinderungen." (Behrens \& Tiedt, 2012, S. 145) Auch Tanz kann somit von besonderem Interesse in der körperbezogenen Arbeit mit Kindern und Jugendlichen mit verschiedenen Verhaltensauffälligkeiten sein. Die Vereinigung dieser beiden Elemente (Kampf und Tanz) in der Capoeira lassen bereits ihr mögliches Potenzial für Jugendliche und junge Erwachsene mit verschiedenen sozialen oder emotionalen Schwierigkeiten erahnen. Und so wird der Capoeira nachgesagt, dass sie es einfacher machen könne, „Prinzipien und Werte zu erwerben, wie Selbstwert, Respekt, Solidarität und Selbstüberwindung" (Abib, 2005, zitiert nach Cavalcante \& Palhares, 2008, S. 107).

Capoeira schult in hohem Maß die Impulskontrolle. Die Handelnden zeigen in ihrer Bewegung: Ich könnte dich jetzt treffen - tue es aber nicht! Durch das gemeinsame Spiel, durch jede Aktion der Gegnerin oder des Gegners, die eine Reaktion erfordert, wird die Aufmerksamkeit trainiert. Die Bewegungsabläufe fördern, wie auch in anderen Kampfsportarten, aber ähnlich vor allem dem Tanz, die Körperbeherrschung. 
In der Capoeira wird darüber hinaus großer Wert auf die Vermittlung und Einhaltung von Regeln, Disziplin und grundlegenden Formen der Höflichkeit sowie Respekt dem Gegenüber gelegt. Bei Menschen mit emotionalen Auffälligkeiten, extrem schüchternem oder depressiv zurückgezogenem Verhalten fördert die Capoeira insbesondere auch die Selbstüberwindung und das Selbstbewusstsein: In den Kreis zu treten und mit einer Partnerin oder einem Partner die eigenen Fähigkeiten und Fertigkeiten vorzuführen, kostet dabei womöglich Überwindung. Getragen von der Musik, dem beständigen Anfeuern der anderen Capoeiristas und dem Wissen um eine baldige Ablösung auf der Bühne ist dies jedoch womöglich einfacher zu schaffen. Gerade dieses Gruppensetting in der Capoeira, der Teilnahme aller an der roda, eröffnet für jeden Menschen unabhängig von physischen oder psychischen Voraussetzungen des ${ }^{\star}$ der Einzelnen maximale Partizipationsmöglichkeiten.

\section{Capoeira é tudo! (Capoeira ist alles!) - Mestre João Pequeno}

Capoeira ist alles! Mit dieser Antwort auf die Frage, was Capoeira denn nun sei, gibt Mestre João Pequeno eine der vielen Definitionen von Capoeira (vgl. Hegmanns, 1998, S. 11). So hat Capoeira tatsächlich sehr viel zu bieten, was für die pädagogische Praxis gerade auch im Hinblick auf musisch-tänzerische aber auch sportliche Angebote von Bedeutung sein kann: Capoeira ist Kampf, Rhythmus und Musik. Durch das Frage- und Antwortspiel der beiden agierenden Körper ist Capoeira auch Tanz, Widerstand und setzt vor diesem Hintergrund Anpassung an ein Gegenüber bewusst ein. Sie geht an die Grenzen und auch darüber hinaus. Capoeira ist Gruppendynamik und schult Respekt, Toleranz, Solidarität und auch Disziplin. Capoeira ist das Erleben von Selbstwirksamkeit und Selbstüberwindung und die Schulung von Impuls- und Körperkontrolle. Capoeira ist der selbst initiierte Aufbruch aus der Exklusion und bietet viele praktische Ideen für die Implementierung inklusiver Strukturen in Sport, Musik, Tanz - vielleicht sogar der Pädagogik im Allgemeinen.

Die sich zunehmend ausbreitenden Capoeira-Schulen ${ }^{9}$ bieten somit die Möglichkeit, Capoeira nicht mehr nur als ein brasilianisches Phänomen zu betrachten, sondern ihr Potenzial auch für die pädagogische oder auch musikalisch-tänzerische Praxis hierzulande zu erkennen und zu nutzen. Das kann bedeuten, selber Capoeira zu erlernen und im schulischen Sportunterricht oder in Tanz- und Musikschulen anzubieten oder aber sich der pädagogischen Möglichkeiten bewusst zu sein und Kinder und Jugendliche - egal ob mit

9 Eine Auflistung der aktiven Capoeira-Vereine in Österreich findet sich bei Doppler (2010). Gerade in Tirol lässt sich feststellen, dass seit diesem Zeitpunkt weitere Akademien dazugekommen sind. 
oder ohne den hier ausgeführten Auffälligkeiten - auf die Qualitäten und das Potenzial der Capoeira aufmerksam zu machen, Kontakte zu Capoeira-Akademien herzustellen und sie gegebenenfalls dorthin zu begleiten. Auf diese Weise könnte die sinnstiftende und verbindende Kraft der Capoeira auch hierzulande zunehmend Anerkennung finden.

\section{Literatur}

Abib, P. (2005). Capoeira angola: cultura popular e o jogo dos saberes na roda. Salvador: EDUFBA. https://doi.org/10.20396/resgate.v12i13.8645622

Baumann, C., Koring, W., Worms, L. \& Aring, R. (1997). Judo mit Mehrfachbehinderten. In M. Weiß \& H. Liesen (Hrsg.), Rehabilitation durch Sport (S. 126129). Marburg: Kilian Verlag.

Behrens, C. \& Tiedt, W. (2012). Bewegung und Tanz als Gegenstand ästhetisch-kultureller Bildung in der Sozialen Arbeit mit Jugendlichen. In M.-S. Meis \& G.-A. Mies (Hrsg.), Künstlerisch-ästhetische Methoden in der Sozialen Arbeit: Kunst, Musik, Theater, Tanz und digitale Medien (S. 145-176). Stuttgart: Kohlhammer.

Capoeira, N. (2008). Capoeira - Kampfkunst und Tanz aus Brasilien. Berling: Weinmann.

Cavalcante, J. C. O. \& Palhares, L. R. (2008). Capoeira in the process of social inclusion. Fiep Bulletin, 78, 107-109.

Döring, K. (2010). Wahrnehmung und Ausdruck in afrobrasilianischen Tanz-Musiktraditionen. Dissertation, Universität Siegen. Verfügbar unter: https://d-nb.info/ 102224034X/34 [17.07.2020].

Doppler, C. (2010). Capoeira - afrobrasilianischer Kampftanz. Verfügbar unter: https://docplayer.org/36068622-Capoeira-ein-afrobrasilianischer-kampftanzzweikaempfe-kraefte-messen-regeln-beachten-verfasst-von-christian-dopplersaalfelden-maerz-2010.html [07.08.2020].

Ferreira, T. (2012). O uso da capoeira como instrumento psicosocial de inclusão. Revista Projeção e doença, 3/2, 32-45.

Freire, P. (1973). Pädagogik der Unterdrückten: Bildung als Praxis der Freiheit. Reinbek: Rowohlt.

Hegmanns, D. (1998). Capoeira - Die Kultur des Widerstandes. Stuttgart: Schmetterling Verlag.

Hoffmann, T. (2018). Inklusive Pädagogik als Pädagogik der Befreiung. In T. Hoffmann, W. Jantzen \& U. Stinkes (Hrsg.), Empowerment und Exklusion: Zur Kritik der Mechanismen gesellschaftlicher Ausgrenzung (S. 19-48). Gießen: Psychosozial-Verlag. https://doi.org/10.30820/9783837973983-17

Höllinger, F. (2007). Religiöse Kultur in Brasilien. Zwischen traditionellem Volksglauben und modernen Erweckungsbewegungen. Frankfurt/Main, New York: Campus Verlag.

Onori, P. (1988). Sprechende Körper. St. Gallen/Köln: Edition diá. 
Palhares, L. R. (2007). Educação e cultura popular: inclusão social pela capoeira. LICERE - Revista Do Programa De Pós-graduação Interdisciplinar Em Estudos Do Lazer, 10 (3), 1-15.

Quinten, S. (2016). Chancen Kultureller Bildung für Kinder und Jugendliche im inklusiven fähigkeitsgemischten Tanz. sportunterricht, 12, 369-373.

Röhrig Assunção, M. (1999). CAPOEIRA - Zur Geschichte einer afro-brasilianischen Kunstform zwischen Anpassung und Widerstand. Verfügbar unter: http://repo sitory.essex.ac.uk/9669/1/capoeira-zur-geschichte-einer-afro-brasilianischenkunstform-zwischen-anpassung-und-widerstand.pdf [06.08.2020].

Scharf da Silva, I. (2004). Umbanda - Eine Religion zwischen Candomblé und Kardezismus - Über Synkretismus im städtischen Alltag Brasiliens. Hamburg: LITVerlag.

Theunissen, G. (2016). Geistige Behinderung und Verhaltensauffälligkeiten. Bad Heilbrunn: Klinkhardt. 



\title{
Zeitgenössisch in Vielfalt unterrichten
}

\author{
Aus der Praxis einer Tanzinitiative
}

\section{Einführung}

Tanz bedeutet für uns allgemein eine Bewegung von Körpern, deren Abfolge in Raum und Zeit geordnet ist. Als Kunstform ist er uns zu wichtig, um ihn allein wohltrainierten, jungen Körpern zu überlassen (vgl. Walser-Wohlfarter \& Richarz, 2018a, S. 220). Mit der von uns geleiteten Initiative tanzfähig wollen wir den Zeitgenössischen Tanz allen zugänglich machen, die tanzen wollen, unabhängig von Alter, Behinderung, Herkunft oder Vorerfahrung. In dieser Anerkennung der menschlichen Vielfalt sehen wir eine Bereicherung für den Tanz. Denn: „Das Tanzen muss einen anderen Grund haben als bloße Technik und Routine. [...] Es geht um das Leben, und darum, für das Leben eine Sprache zu finden." (Bausch, 2016, S. 317-318)

Um Körperlichkeiten in ihrer ganzen Vielfalt in den Tanz einbeziehen zu können, nehmen wir Abstand vom Normalen. Das beinhaltet: Es geht uns nicht um die Anpassung an ein Bewegungsideal, das kulturell als richtig oder schön angesehen wird. Es geht uns auch nicht um eine Dichotomie von gesund und behindert, jung und alt, trainiert und untrainiert, sondern vielmehr um eine Pluralität im Tanz. Sie zu verwirklichen heißt für uns, dass es unterschiedliche, aber gleichwertige Formen des Tanzes gibt, die sich jeweils aus der Körperlichkeit der Tänzer*innen ergeben. Wenn wir mit behinderten Menschen tanzen, grenzen wir uns von Angeboten ab, bei denen Behinderte mitmachen dürfen, weil die Inklusion es verlangt, und kritiklos alles beklatscht wird, was sie hervorbringen, aber auch von solchen, wo sie zum moralischen oder finanziellen Vorteil der Nicht-Behinderten beteiligt werden oder deren Inspiration dienen sollen. Jenseits von Wohltätigkeit und Ausbeutung fordern wir die bei uns Tanzenden darin, die Einzigartigkeit ihrer Person in den Tanz einzubringen und Tänze entstehen lassen, bei denen ihre Körperlichkeit etwas Neues, bisher nicht Dagewesenes bedingen kann. Für unseren Ansatz haben wir den Begriff eines Zeitgenössischen Tanzes in körperlicher Vielfalt geprägt. Neben der künstlerischen Produktion ${ }^{1}$ und der tanzwissenschaftlichen

1 Von 2011 bis 2019 sind fünf Produktionen unter dem Namen von tanzfähig entstanden, nämlich drei Bühnenstücke, ein Video-Tanz und ein Dokumentarfilm. Außerdem laden wir zu öffentlichen Proben ein und wir veranstalten Aufführungen mit Tanzimprovisation. 
Reflexion ${ }^{2}$ blicken wir in der Initiative tanzfähig auf eine mehr als zwölfährige Unterrichtserfahrung zurück.

\section{Vielfältiges Unterrichten: unsere Formate, unsere Intentionen}

Die tanzpädagogischen Angebote von tanzfähig sind offen für alle, die sich körperlich ausdrücken wollen. Neben Erwachsenen unterrichten wir Kinder, teils mit ihren Eltern, teils in Zusammenarbeit mit Schulen. Bei der Auswahl von Veranstaltungsorten bemühen wir uns um eine größtmögliche Barrierefreiheit. ${ }^{3}$ In den Ankündigungen verweisen wir auf die Zugänglichkeit, die von uns gewährleistet werden kann. Das heißt unter anderem, vorhandene Behindertenparkplätze aufzuführen, das Studio ausdrücklich als rollstuhlgerecht zu benennen oder die Übersetzung in Deutsche Gebärdensprache zu erwähnen, falls diese Voraussetzungen gegeben sind. Unabhängig davon, ob wir selbst anleiten oder ob wir andere dafür gewonnen haben, ein Angebot anzuleiten, laden wir für die Teilnahme jeweils mit den Worten ein: „Tänzerische Vorerfahrungen, kulturelle Prägungen und Behinderungen jeglicher Art sind uns willkommen, aber nicht erforderlich." (Walser-Wohlfarter \& Richarz, 2018a, S. 222)

Bei den Angeboten, die wir selbst anleiten, ist an erster Stelle das wöchentliche Tanztraining in körperlicher Vielfalt zu nennen. In Berlin führen wir es fortlaufend seit 2007 durch, seit 2017 auch in Vorarlberg bzw. in Tirol. Nachdem es in den ersten Jahren ein fähigkeitsgemischter Unterricht auf Grundlage von DanceAbility ${ }^{4}$ gewesen ist, folgen wir inzwischen keinem unmittelbar auf Behinderung bezogenen Ansatz mehr. Wir vermitteln jetzt zeitgenössischen Tanz mit einem Schwerpunkt auf Improvisation, wobei wir darauf achten, dass alle, die im Raum sind, sich daran beteiligen können. ${ }^{5}$ Neben der Schulung tänzerischer Fertigkeiten bietet das Training uns selbst und allen anderen Teilnehmenden eine Gelegenheit zur Bewegungsforschung. Einige nehmen daran

22017 ist die tanzwissenschaftliche Konferenz Tanz Körper Erweiterung | Stretching the Physicality of Dance über die künstlerische Bedeutung von körperlicher Vielfalt im Tanz von uns kuratiert und zusammen mit dem Dachverband Tanz Deutschland und dem Hochschulübergreifenden Zentrum Tanz Berlin organisiert worden. Auch haben wir zu unserem tänzerischen Ansatz publiziert und die Tätigkeit der Initiative auf verschiedenen Tagungen im In- und Ausland vorgestellt.

3 Eine Übersicht, was für die Barrierefreiheit von Kulturveranstaltungen allgemein zu beachten ist, findet sich z. B. in Kultur für Alle (2018).

4 DanceAbility ist eine von Alito Alessi seit den 1980er Jahren entwickelte Methode, die ausgehend von der Kontaktimprovisation das gemeinsame Tanzen von Menschen mit und ohne Behinderungen ermöglicht.

5 In Abschnitt 4 führen wir genauer aus, wie und was wir unterrichten, und diskutieren es unter tanzpädagogischen Gesichtspunkten. 
über längere Zeit regelmäßig teil, andere nur kurz, und immer wieder kommen auch Gäste hinzu, die selbst erleben wollen, was es heißt, in körperlicher Vielfalt zu tanzen. Neben dem Training bieten wir Workshops an, die von uns selbst veranstaltet werden oder zu denen wir als Referierende eingeladen werden. So haben wir in den verschiedensten Zusammenhängen unterrichtet, vom Jugendtanzfestival bis zum Bürger-Café, von der Hochschule bis zur Selbsthilfegruppe.

Wenn wir andere einladen, bei tanzfähig zu unterrichten, wählen wir sie danach aus, ob sie schon länger mit körperlicher Differenz im Tanz umgehen und ob sie unsere Angebote inhaltlich zu erweitern vermögen. So haben wir Workshops mit Jess Curtis ${ }^{6}$, Adam Benjamin ${ }^{7}$ und matanicola ${ }^{8}$ veranstaltet, deren Herangehensweise wir schätzen und welche auch uns geprägt hat; und im Training sind Unterrichtseinheiten zu Gaga ${ }^{9}$, Physical Theatre ${ }^{10}$, barrierefreiem Yoga ${ }^{11}$, nichtvisuellem Tanz ${ }^{12}$ u. a. dazugekommen. Für manche, die dabei unterrichtet haben, ist es eine von ihnen selbst gesuchte Herausforderung gewesen, ihre Technik erstmals unter den Bedingungen einer körperlichen Vielfalt anzuwenden. Gerne laden wir außerdem erfahrene Tänzerinnen und Tänzer mit Behinderung ein, bei uns zu unterrichten.

Die verschiedenen Formate, die wir anbieten, sind inklusiv ausgerichtet. Vorab fragen wir körperliche Bedingungen der Teilnehmer*innen ebenso we-

6 Jess Curtis, künstlerischer Leiter von Gravity in Berlin und San Francisco, bezieht seit langem Darsteller mit verschiedenen Fähigkeiten und auch mit Behinderungen in seine Produktionen ein. Er versucht, Tanzdarbietungen auch einem blinden oder gehörlosen Publikum zugänglich zu machen.

7 Adam Benjamin, Choreograf und ehemaliger Hochschullehrer, hat 1990 in London die integrierte Tanzkompagnie Candoco mitbegründet und ein grundlegendes Buch über Tanz und Behinderung geschrieben.

8 Das Berliner Choreografen-Duo Matan Zamir und Nicola Mascia (matanicola) gehört zum Freundeskreis von Sasha Waltz. Sie haben Tanzstücke mit gehörlosen und mit lernbehinderten Darstellern gemacht sowie sich mit Parkinson-Tanz befasst.

9 Gaga ist eine von dem israelischen Choreografen Ochad Nacharin entwickelte Tanztechnik. Bei ihr soll der Körper nicht wie üblich durch Figuren geformt, sondern von innen heraus an neue Grenzen gebracht werden.

10 Beim Physical Theatre werden Geschichten nicht durch einen Text, sondern durch den Körper, seinen Ausdruck und seine Bewegung erzählt.

11 Beim barrierefreien Yoga passt der Lehrende seine Anleitung individuell an die anwesenden Kursteilnehmer*innen und ihre körperliche Konstitution an, um dadurch unabhängig von körperlichen Einschränkungen Yoga allen zugänglich zu machen.

12 Der nichtvisuelle Tanz ist von Zwoisy Mears-Clarke entwickelt. Darunter ist ein Tanz zu verstehen, der zwischen Tänzer(*inne)n und Zuschauer( ${ }^{*}$ inne)n nicht visuell, sondern somatosensorisch vermittelt wird. Er findet im Dunkeln statt. Die Zuschauer*innen , sehen' mit ihrem Körper, der für die Tänzer*innen zur Tanzfläche geworden ist. 
nig ab wie ihr Alter oder ihre Herkunft. So sind wir bisweilen überrascht, wer kommt, manche wiederum teilen von sich aus mit, wenn sie Besonderes brauchen. Über die Zeit hinweg sind zum Tanzen bei uns gewesen: Rollstuhlfahrende mit und ohne Elektroantrieb, Spastiker und Tirolerinnen, Tanzerfahrene und Anfänger ${ }^{*}$ innen, vier Polionauten ${ }^{13}$, drei Chinesinnen, Ältere über 60 und eine Jugendliche mit 14 Jahren, Homo-, Hetero- und Transsexuelle, Gläubige und Ungläubige, Deutsche, weniger Männer und mehr Frauen, eine Gehörlose, eine Blinde und eine Taubblinde, mehrere Sehbehinderte und Lernbehinderte, Mütter, Väter und andere Betreuer*innen, eine Polin, ein Schwarzafrikaner und viele mehr. ${ }^{14}$ Wir zählen sie absichtlich in dieser Weise auf, um die Vielfalt $\mathrm{zu}$ veranschaulichen, der wir im Studio begegnen. Sie alle bringen ihre unterschiedlichen Erwartungen an und Erfahrungen mit Tanz ebenso mit wie ihre verschiedenen Körperlichkeiten und Einstellungen sowohl zu ihrem Körper als auch zu dem der anderen. Bisweilen arbeiten wir auch in exklusiven Formaten. So gibt es unter denen, die wir bis jetzt angeleitet haben, eine Gruppe nur für Frauen im Alter von 60+ oder ein Schulprojekt mit taubblinden Kindern und Jugendlichen. Aber es sind nie Menschen mit Behinderungen, die ausgeschlossen sind, und auch dabei sind die Teilnehmenden so unterschiedlich, dass selbst in diesen exklusiven Formaten eine Vielfalt besteht, die für den Tanz aufgegriffen werden kann. Im Rahmen des Projekts Making A Difference ${ }^{15}$ haben wir erstmals Workshops mitveranstaltet, die behinderungsspezifisch ausgeschrieben waren, nämlich einen für Gehörlose, einen für Sehbehinderte und einen für körperlich Behinderte; bei letzterem ist beispielsweise erkundet worden, wie deren alltägliche Hilfsmittel wie Krücken, Orthesen oder Rollstühle genutzt werden können, um Bewegung im Tanz entstehen zu lassen.

Unsere verschiedenen Angebote geben Menschen mit unterschiedlicher Körperlichkeit, dabei vor allem mit körperlichen Behinderungen, die andernorts oft fehlende Gelegenheit, sich tänzerisch zu entwickeln. Wir bemühen uns, sie auch darüber hinaus zu fördern. Durch unsere Beteiligung an dem

13 Polionauten: Selbstbezeichnung von Personen, die eine Erkrankung an Kinderlähmung (Polio) überlebt haben.

14 Um Vielfalt auch im Text wiederzugeben, wählen wir hier und an anderen Stellen verschiedene Formen der gendergerechten Sprache mit ihren unterschiedlichen Bedeutungen. Wir verwenden auch bewusst das generische Maskulinum, da Vielfalt sich nicht nur auf das Geschlecht bezieht, sondern auch auf Alter, Herkunft oder Behinderung, und wir benutzen das Femininum, um das Besondere auszudrücken.

15 Bei dem Projekt Making A Difference arbeiten von 2018 bis 2021 unter Leitung der Sophiensäle GmbH acht Berliner Institutionen des zeitgenössischen Tanzes, u. a. die tanzfähig Wohlfarter Richarz GbR, zusammen, um über verschiedene Module Performer*innen und Choreograf $\left({ }^{*}\right.$ inn $)$ en mit körperlichen und sensorischen Behinderungen vermehrt am zeitgenössischen Tanz zu beteiligen. Das Projekt wird zu gleichen Teilen von Tanzpakt Deutschland und dem Berliner Senat für Kultur und Europa gefördert. 
EU-Projekt Moving Beyond Inclusion ${ }^{16}$ haben wir zweimal vier Tänzer*innen benennen können, die in zwei choreographischen Labs in Schweden und Italien eine hochwertige Fortbildung erhalten haben. Außerdem ermutigen wir unsere Tänzerinnen und Tänzer, auch dann Tanzklassen an anderen unterrichtenden Institutionen zu besuchen, wenn sie nicht ausdrücklich auf behinderte Teilnehmer*innen eingestellt sind. Durch unsere Beteiligung am Projekt $\mathrm{Ma}$ king A Difference sind wir über vier Jahre hinweg in der Lage, jährlich sechs Interessierten einen Gutschein in Höhe von $500 €$ zukommen zu lassen, mit dem sie Tanzangebote ihrer Wahl besuchen können. Obwohl es sicher nicht nur als ein Erfolg unseres Unterrichts anzusehen ist, freuen wir uns, wenn ehemalige Teilnehmer ${ }^{*}$ innen außerhalb unserer Initiative an künstlerischen Produktionen beteiligt sind. ${ }^{17}$

Schließlich ist uns auch daran gelegen, Tanzpädagoginnen und Tanzpädagogen einen Tanz in körperlicher Vielfalt nahe zu bringen. Wiederum im Rahmen des Projekts Making A Difference sind wir an Fortbildungen für Lehrende beteiligt. In einer sogenannten Forschungsgruppe wird, indem die Teilnehmenden aus ihren Erfahrungen berichten, untersucht, wie inklusives Unterrichten im Tanz gelingen kann, welche Schwierigkeiten sich dabei ergeben und wie sie überwunden werden können. In einem eigens entwickelten WorkshopFormat bekommen die Unterrichtenden nach einem zweistündigen Unterricht von den Teilnehmer(*inne)n zurückgemeldet, in wie weit sie wirklich inklusiv angeleitet haben. Das beinhaltet nicht nur, dass Behinderte sagen, wie sie sich einbezogen gefühlt haben, sondern auch Nicht-Behinderte, wie ansprechend für sie der Unterricht gewesen ist.

16 Durch die Zuwendung von EACEA im Rahmen des EU-Programms Creative Europe und die Co-Finanzierung des Berliner Senats für kulturelle Angelegenheiten hat die Initiative tanzfähig finanzielle Unterstützung erhalten, um im Austausch mit sechs europäischen Partnern unter Führung von Candoco Dance Company aus London ihre Strukturen zu professionalisieren, Tänzerinnen und Tänzer mit Behinderungen hochwertig zu qualifizieren und in Deutschland den Diskurs über Tanz und Behinderung anzuregen.

17 Aus den letzten Jahren können beispielhaft die Tanzstücke Reverberations von Michael Turinsky, Bondage Duell von Dasniya Sommer und Silke Schönfleisch sowie Atlas Berlin von Ana Borralho und João Galante genannt werden. 


\section{Exkurs: der geschädigte und der behinderte Körper ${ }^{18}$}

Wenn im Sinne der Inklusion Menschen mit Behinderungen ${ }^{19}$ in den Tanz einbezogen und ihnen die herkömmliche Tanzausbildung vermehrt zugänglich gemacht werden soll, erscheint es uns angebracht, genauer zu bestimmen, was es heißt, mit einem Körper zu leben, der als geschädigt oder behindert gilt. Seitdem 2006 die UN-Behindertenrechtskonvention verabschiedet und danach in den meisten Staaten ratifiziert worden ist, erfahren Behinderte eine hohe rechtliche Anerkennung. Ihr Anspruch auf volle Teilhabe am gesellschaftlichen Leben kann nicht mehr bestritten werden und erstreckt sich auch auf den kulturellen Bereich. Welchen Stellenwert eine Gesellschaft den Menschen einräumt, deren Körperlichkeit als anders und von der Norm abweichend angesehen wird, sagt immer auch etwas über die Gesellschaft aus (vgl. Köberl, 2014, S. 76). Dazu gehört, dass die Welt- und Lebenserfahrung, die sich aus einer Behinderung ergibt, sozial wenig wertgeschätzt wird. Das besondere Wissen, das behinderte Menschen aus ihrer Körperlichkeit gewinnen, ist aus dem allgemeinen Diskurs, was menschliches Leben bedeutet, weitgehend ausgeschlossen (vgl. Wendell, 1996, S. 64-66). Gerade im Zuge ihrer vermeintlichen Normalisierung werden ihre Erfahrungen bagatellisiert (vgl. Willemsen, 2014, S. 11). Die Ausgrenzung von sogenannten Anderen in der Kultur gibt sich dabei oft, wenn auch verhohlen, als ästhetisches Urteil (vgl. Siebers, 2009, S. 20).

Als Behinderung wird eine dauerhafte und sichtbare Abweichung im körperlichen, geistigen oder seelischen Bereich bezeichnet, der allgemein ein negativer Wert beigemessen wird (vgl. Cloerkes, 2007, S. 8). Während im medizinischen Modell Behinderung allein auf den geschädigten Körper zurückgeführt wird, werden im sozio-kulturellen Modell gesellschaftliche Bedingungen und kulturelle Gepflogenheiten im Umgang mit dem geschädigten Körper herangezogen, um Behinderung zu bestimmen (vgl. Dederich, 2007, S. 23-28; Waldschmidt, 2005). Entsprechend der Definition von Behinderung erscheint der Behinderte stereotyp entweder als bedauernswertes Opfer, dem ein ungerechtes Schicksal übel mitgespielt hat, oder als beinahe übermensch-

18 Der Abschnitt beruht in wesentlichen Teilen auf zwei unveröffentlichten Manuskripten (vgl. Richarz, 2018 a,b).

19 In den britischen Disability Studies wird von behinderten Menschen gesprochen, um $\mathrm{zu}$ verdeutlichen, dass das Behindert-Sein selbstverständlich zur Identität dazugehört und sie geprägt hat, in den amerikanischen dagegen von Menschen mit Behinderungen, um zu verdeutlichen, dass trotz der Behinderung das Mensch-Sein an erster Stelle steht. Wir verwenden die Bezeichnungen abwechselnd, ebenso die Bezeichnung Behinderte, die sich seit den 1970er Jahren bis in die 1990er im deutschen Sprachraum verbreitet hat. Auch wenn in letzter Zeit häufig von Handicap oder Beeinträchtigung gesprochen wird, verwenden wir den Begriff Behinderung, um auf den sozialen Anteil zu verweisen, der zur körperlichen Schädigung dazu gehört. 
licher Held, der trotz aller Widrigkeit sein schweres Los erfolgreich meistert (vgl. Maskos, 2004). Zwar lassen sich verschiedene Arten von Behinderung ausmachen, doch gemeinsam ist ihnen, dass die als behindert Bezeichneten nicht in der Lage sind, die Körper- und Affektkontrolle zu erreichen, die heutzutage in den von der Aufklärung geprägten westlichen Gesellschaften verlangt wird. Dadurch werden sie wie alle, die nicht weiß, heterosexuell, männlich und mittleren Alters sind, zu Anderen. Mit ihrer Gegenwart überschreiten sie die unsichtbare Schwelle, die das sozial Schickliche vom Peinlichen trennt. In einer Zeit, in der es mehr und mehr ein Zeichen von Unwissenheit und mangelnden finanziellen Ressourcen ist, noch einen Körper zu haben, der nicht den Standards der Schönheitsindustrie entspricht (vgl. Ziemer, 2008, S. 121) und die Bilder der Idealkörper allgegenwärtig sind (vgl. Vernaldi, 2007), erregen sie Aufmerksamkeit und werden beobachtet und bewertet, sobald sie in Erscheinung treten. Sie faszinieren, weil sie mit ihrer Körperlichkeit einen sonst verwehrten Blick in menschliche Abgründe und Abweichungen des Seins erlauben, und verunsichern, weil sie die Frage aufwerfen, inwieweit eine körperliche Unversehrtheit wirklich beständig ist (vgl. Mitchell \& Snyder, 2000, S. 15-45). Schnell geraten sie unter Druck, sich rechtfertigen zu müssen, dass sie überhaupt leben, weil sie mit ihrem angeblich sinnlosen Da-Sein mehr nehmen als geben würden. Doch unausgesprochen beruhigt ihr Vorhandensein diejenigen, die in der Hierarchie körperlicher Abweichungen höher stehen und als nichtbehindert gelten. Da behinderte Menschen das Scheitern am gesellschaftlichen Ideal so sichtbar verkörpern, brauchen die anderen nicht zu befürchten, wegen ihrer Körperlichkeit gleich jenen ihre soziale Anerkennung zu verlieren.

Treffen trotz aller materiellen und immateriellen Barrieren Nicht-Behinderte und Behinderte zusammen, gilt es als Aufgabe der Letzteren, mit dem aufkommenden Unbehagen umzugehen (vgl. Goffman, 1967, S. 143-153). Wenn sie der unausgesprochenen Erwartung des dankbaren und schuldbewussten Behinderten nicht entsprechen, stoßen sie rasch auf Feindseligkeit. So wie so kann die Behinderung wie ein weißer Elefant im zwischenmenschlichen Raum stehen: In Gegenwart des behinderten Körpers wird alles andere nicht wahrgenommen, aber im Gespräch wird die Behinderung nicht behandelt; oder es wird besonders betont, wie wichtig die Mehrheitsnormen von Autonomie, Effizienz, Ökonomismus oder Ästhetik sind (vgl. Rommelspacher, 1999, S. 205). Im Alltag wird vermieden, Behinderten von Mensch zu Mensch zu begegnen und ein Austausch über die verschiedenen Sichtweisen der Wirklichkeit kommt nicht zustande. Darin sind sich alle einig, dass der behinderte Körper mit seinen besonderen Grenzen und Möglichkeiten das Selbst und sein Erleben einschränkt, statt wahrzunehmen, welche Erkenntnisse über das Leben ein behinderter Körper vermitteln kann, die sonst gar nicht möglich wä- 
ren. Der behinderte Körper bleibt vielmehr ein Objekt der Kränkung und des Schmerzes und den Spezialisten des Gesundheitswesens und den Experten des Sozialwesens überantwortet, damit sie ihn mit den ihnen zur Verfügung stehenden Ressourcen verbessern. Er bleibt aber auch ein heimliches Objekt der Begierde und der Lust, das sogenannte Devotees, Pretenders und Wannabees ${ }^{20}$ als sexuell attraktiv erleben (vgl. Bruno, 1997).

So verschieden die körperlichen Schädigungen sind, die dann die geistigen, seelischen, sensorischen oder körperlichen Behinderungen bedingen, so ist ihnen doch gemeinsam, dass sie einen Bezug zwischen Körper und Umwelt entstehen lassen, der von der Norm abweicht. ${ }^{21}$ Dieser Zusammenhang von körperlicher Schädigung und einem von der Norm abweichenden Bezug zwischen Körper und Umwelt kann in folgenden Bereichen (vgl. Richarz, 2018a, S. 1-9) näher beschrieben werden:

- Die Schädigung kann die Wechselwirkung eines Körpers mit seiner Umwelt betreffen. Diese Wechselwirkung weicht von der Norm ab, wenn Gesprochenes nicht gehört werden kann, wenn eine zweite Hand zum festen Zupacken fehlt, wenn dreimal in der Woche das Blut in der Dialyse gewaschen werden muss oder wenn ein Geschehen affektiv nicht verarbeitet werden kann. Diese Wechselwirkung weicht aber auch von der Norm ab, wenn der Blinde sich am Regen erfreut, weil er erst so die Landschaft erfassen kann, die vor ihm liegt, oder wenn sich für die vom Hals abwärts Gelähmte die Welt der Fantasie auftut, weil sie mit ihrem Körper in der Wirklichkeit nicht mehr handeln kann.

- Die Schädigung kann die Bedürfnisse betreffen, die ein Körper hat, um Gleichgewicht und Wohlbefinden zu erreichen. Diese Bedürfnisse weichen von der Norm ab, wenn es einen Beutel braucht, um den Urin zu sammeln; die Finger, um einen anderen Körper zu ertasten; oder einen Ruheraum, um bei sich zu bleiben, wenn das Gehirn von Informationen überflutet wird.

- Die Schädigung kann die Art betreffen, wie ein Körper willentlich gesteuert wird. Diese Art weicht von der Norm ab, wenn nicht zu beeinflussende Spasmen durch den Körper laufen; oder wenn der Speichel nicht im Mund behalten werden kann. Sie weicht aber auch von der Norm ab, wenn der

20 Devotees: Personen, die durch körperliche Behinderungen erregt werden; Pretenders: Personen, die ihr Äußeres verändern, um behindert zu erscheinen; Wannabees: Personen, die ihre Körperlichkeit verändern wollen, um behindert zu sein.

21 Eine bestimmte Körperlichkeit als geschädigt oder von der Norm abweichend zu bezeichnen, wird hier beibehalten, weil es die Definition von Behinderung aufgreift. Aber genau wie Behinderung sind auch Schädigung oder Abweichung von der Norm soziokulturelle Konstrukte, welche die Vielfalt menschlichen Lebens begrenzen und bestimmte Formen als unerwünscht ausgrenzen. 
Blutzucker von der Insulinpumpe reguliert wird; oder wenn ein Rollstuhl benutzt wird, um von A nach B gelangen zu können.

- Die Schädigung kann die Bewusstheit betreffen, mit der der Körper erlebt wird. Diese Bewusstheit weicht von der Norm ab, wenn geringe Belastungen zu Atemnot führen und das Herz spürbar schlagen lassen; oder wenn anhaltender Schmerz den Rücken spüren lässt.

- Die Schädigung kann den Umgang mit Raum und Zeit betreffen. Dieser Umgang weicht von der Norm ab, wenn der Raum, der mit dem Körper erfasst werden kann, nicht über die Länge der beiden Arme hinausgeht, mit denen er berührt werden kann, oder wenn sich die Entfernungen in ihm scheinbar vergrößert haben, weil jeder Schritt Mühe kostet; wenn durch eine schwere Erkrankung die absolute Grenze der Zeit erfahren worden ist; wenn sie relativ als begrenzt empfunden wird, weil alles länger braucht; wenn sie dem vorgegebenen Rhythmus einer Beatmungsmaschine unterliegt; oder wenn sie sich endlos dehnt, weil sie nicht mit Handeln gefüllt werden kann.

- Die Schädigung kann die Passung zur Umwelt betreffen. Diese Passung weicht von der Norm ab, wenn sie sich nicht im Heranwachsen altersgemäß entwickelt hat, sondern wenn durch orthopädische Hilfsmittel, Operationen, therapeutische Maßnahmen oder Einnahme von Medikamenten versucht werden muss, sie zumindest annähernd zu erzielen.

- Die Schädigung kann die Gefühle betreffen, die ein Körper vermittelt. Diese Gefühle weichen von der Norm ab, wenn sie durch andere Inhalte als bei Nicht-Behinderten ausgelöst werden. Die Vorstellung, dass die vertraute Bezugsperson nicht in der Nähe ist, macht nicht beherrschbare Angst; es macht Freude, wenn die Medikamente wirken und die Muskelstarre neuer Beweglichkeit weicht; der Anblick des hinkenden Gangs im Spiegel kann beschämen; wahrzunehmen, über welch körperlichen Fertigkeiten andere verfügen, und zu erkennen, sie selbst nie ausüben zu können, macht traurig oder neidisch.

\section{Diskussion unserer Erfahrungen}

Durch eine Vielzahl fähigkeitsgemischter Tanzgruppen (vgl. Quinten \& Schwiertz, 2014) gibt es eine reichhaltige Erfahrung, wie Menschen mit Behinderungen in den Tanz einbezogen werden können. Wenn Tanz als geordnete Bewegung von Körpern in Raum und Zeit verstanden wird, vermögen Tänzerinnen und Tänzer mit einem Körper, der als behindert oder geschädigt gilt, im Studio und auf der Bühne den Tanzkörper über das Gewohnte hinaus zu erweitern. In der Ausbildung können sie neue Erfahrungen bedingen, 
Vertrautes durcheinanderbringen und zum Nachdenken anregen, was Tanz ausmacht (vgl. Benjamin, 2002, S. 8). Bei Aufführungen vermitteln sie, dass ein Tanz sich zwar aus der Körperlichkeit einer Tänzerin oder eines Tänzers ergibt, aber nicht durch sie begrenzt ist (vgl. Cooper Albright, 1997, S. 58). Einem Publikum können sie bewusst machen, wie andere Körper sozial wahrgenommen werden (vgl. McGrath, 2013, S. 9), und dazu beitragen, die kulturelle Norm zu hinterfragen, wie ein Körper auszusehen und zu empfinden hat (vgl. Hermans, 2016, S. 161).

Wenn Tanz im Sinne des klassischen Balletts aber mit Sprüngen und scheinbar müheloser Körperbeherrschung gleichgesetzt wird, ist es unvorstellbar, dass Behinderte, die infolge ihrer abweichenden Körperlichkeit solch Virtuosität vielfach nicht aufweisen, überhaupt tanzen können. Von der Musikschule für Kinder bis zur Hochschule (vgl. Senk, 2015) sind Institutionen, die im Tanz ausbilden, meist nicht darauf eingestellt, Menschen mit Behinderungen aufzunehmen. Oft noch dem anerkannten Ideal der Körper- und Affektkontrolle verpflichtet, verfügen sie weder über barrierefrei zugängliche Studios noch haben sie Kriterien, an Hand derer sie die Eignung von behinderten Bewerberinnen und Bewerbern beurteilen können, noch berücksichtigen ihre Lehrpläne und Prüfungsordnungen deren besondere Körperlichkeit. Auch ihre Dozentinnen und Dozenten haben oft wenig Umgang mit Behinderten im allgemeinen und behinderten Tänzer $\left({ }^{*}\right.$ inne)n im Besonderen gehabt, so dass sie vielfach gar nicht wissen, wie sie sie angemessen unterrichten können.

Um an unserem Beispiel zu verdeutlichen, wie Menschen mit Behinderungen in den Tanz einbezogen werden können, erläutern wir unseren Ansatz eines Zeitgenössischen Tanzes in körperlicher Vielfalt unter fünf Gesichtspunkten. ${ }^{22}$ Indem wir uns auf Literatur über Tanz und Behinderung beziehen, versuchen wir, unseren Ansatz in einen tanzpädagogischen Zusammenhang zu stellen. Dabei fließen auch Ergebnisse der oben erwähnten Forschungsgruppe zu inklusivem Unterrichten ein.

\subsection{Haltung der Unterrichtenden}

Die innere Haltung der Lehrer*innen in den Klassen und der Leiter*innen von Institutionen ist entscheidend, um Menschen mit Behinderungen erfolgreich in einen Tanzunterricht einzubeziehen. Dazu gehört, die eigene Einstellung immer wieder zu überprüfen, um für die eigene kulturell-gesellschaftli-

22 In einer inhaltlichen Untersuchung der Literatur zur inklusiven Tanzdidaktik und -methodik für den Schulsport finden sich die fünf Kategorien Haltung, Arbeitsweisen im inklusiven Tanzunterricht, Rahmenbedingungen, Anforderungen an die Lehrenden und Kommunikation (vgl. Albert, 2018). 
che Prägung und die damit verbundenen Werthaltungen und Normen sensibel zu bleiben (vgl. Albert, 2018, S. 23). Für das Anliegen, Tanzklassen auch behinderten Menschen zugänglich zu machen, werden meist politische, pädagogische oder künstlerische Motive aufgeführt. So heißt es: Damit werde der gesetzlich verlangten Inklusion genüge getan und das Recht von behinderten Menschen auf Teilhabe umgesetzt. Behinderte zögen daraus einen Nutzen für ihre Persönlichkeitsentwicklung und bereicherten durch ihre Anwesenheit Nicht-Behinderte. Gerade behinderte Künstler*innen würden einen wesentlichen Beitrag leisten, im Tanz die ganze Tiefe und Weite menschlicher Erfahrung auszuloten, wenn sie die einzigartigen Gegebenheiten ihres Körpers als Quelle ihrer Kunst einbrächten (vgl. Williams, 2014, S. 9; Benjamin, 2002, S. 6). $\mathrm{Zu}$ ergänzen sind Motive, die mehr aus dem Persönlichen stammen. Behinderte in den Tanz einzubeziehen ist denen ein Anliegen, denen das Leben mit ihnen aus der Familie oder dem Bekanntenkreis vertraut ist oder die über eigene Behinderungserfahrung verfügen. Außerdem erhoffen sich manche dadurch ein Mehr an Selbstwert, sozialer Anerkennung, künstlerischer Anregung oder finanzieller Förderung.

Für uns ist es eine politische Haltung, bestimmte Körper für den Tanz als geeignet zu wählen und andere von Studio oder Bühne auszuschließen. Wohlwollend Behinderte mitmachen zu lassen, genügt dabei unseres Erachtens nicht. Ohne zumindest zu ahnen, was es bedeutet, behindert zu leben, mangelt es an Achtsamkeit dafür, was Menschen mit Behinderungen im Studio oder auf der Bühne brauchen oder was sie in den Tanz einbringen können. Dann kann sich leicht wiederholen, was auch im Alltag geschieht, wenn Behinderte und Nicht-Behinderte zusammentreffen. Wir sehen die Verschiedenheit in Person, Vorerfahrung, Herkunft, Alter oder Behinderung für den gemeinsamen Tanz als grundlegend an, und zugleich kann sie in ihm aufgehoben sein. Was manche als krank, behindert, alt, fremd oder gestört ansehen, ist für uns Gelegenheit, den tänzerischen und persönlichen Ausdruck zu erweitern. Wir sind überzeugt, dass nicht nur das Verspielte, das Kraftvolle und das Sinnliche, sondern auch das Verletzbare, das Unvollkommene und das Behinderte Tanz entstehen lassen. Im Unterricht wollen wir Tänzerinnen und Tänzern mit unterschiedlichen körperlichen, geistigen und seelischen Voraussetzungen die Möglichkeit geben, für ihre Kunst voneinander zu lernen und miteinander zu wachsen. Doch wollen wir nicht einen Unterricht für sie, sondern mit ihnen machen. Auch unsere Haltung, die dabei zum Tragen kommt, hat sich aus Persönlichem ergeben, nämlich dem selbstvergessenen Spiel mit dem Nachbarskind, das wegen eines Down-Syndroms als behindert angesehen worden ist, oder dem nicht so spielerischen Umgang mit den Folgen einer kindlichen Polio, wenn sie den Alltag wieder einmal behindert haben. 


\subsection{Die Zielgruppe eines inklusiven Unterrichts}

An einem Tanzunterricht, der Menschen mit Behinderungen einbezieht, nehmen auch Nicht-Behinderte teil. Was sie dazu bringt, gerät oft aus dem Blick. Doch wenn sie nicht bereit sind, sich auf eine körperliche Vielfalt einzulassen, findet Inklusion nicht statt. Nach dem, was wir gehört haben, kann Nicht-Behinderten, die ein inklusives Angebot besuchen, daran gelegen sein, eine schon bestehende umfassende Tanzerfahrung in der Begegnung mit anderen Körperlichkeiten zu erweitern. Sie wollen für sich herausfinden, wie durch körperliche Beschränkung eine Freiheit im Tanz erreicht werden kann, oder allgemein erforschen, was Tanz ist. Sie suchen nach Formen der zwischenmenschlichen Begegnung im Tanz und befassen sich damit, wie durch Tanz Menschen in ihrer Verschiedenheit zusammengebracht werden können. ${ }^{23}$ Andere, die sich in ihrem Körper unsicher sind, finden es einfacher, sich auf das Tanzen einzulassen, wenn es unter Bedingungen geschieht, die Vielfältiges willkommen heißen. Doch es gibt auch die andere Seite, bei der die Angst überwiegt: Manche melden sich an, bisweilen auch mehrfach, und erscheinen doch nicht. Eine junge Frau hat sich nach einem kurzen Blick in das Studio mit den Worten wieder umgedreht: „Das sind mir zu viele Rollstühle!“

Der wirklich geschädigte Körper von Menschen mit Behinderungen kommt im Tanz kaum vor (vgl. Hermans, 2016, S. 160). Das liegt vermutlich nicht nur daran, dass die Angebote nicht offen sind und es damit an den Möglichkeiten des Trainings fehlt, sondern sich im Tanz körperlich zu zeigen verlangt auch von Menschen mit Behinderung Mut. Obwohl sie es gewohnt sind, in ihrem Alltag angestarrt zu werden, und Behinderung als radikalste Form von Performance verstanden werden kann (vgl. Sandahl \& Auslander, 2005, S. 2), die ohne schützenden Rahmen einer Bühne tagtäglich stattfindet, trauen sich viele nicht von sich aus in ein Studio bzw. müssen eigens und wiederholt dazu eingeladen werden. Da Behinderten seit über 200 Jahren vermittelt worden ist, dass sie nicht tanzen können, scheint sich diese Überzeugung in ihnen selbst festgesetzt zu haben.

Auch bei unseren Teilnehmerinnen und Teilnehmern haben sich von klein auf Biografie und Kultur mit ihrem Verständnis von Behinderung und An-

23 Nachdem der argentinische Fotograf Lucas Gonzàlez Canosa eine unserer Klassen besucht hat, fasst er seinen Eindruck zusammen: „Wenn man die sozialen Konstruktionen abzieht, was ein Tänzer sein sollte, sieht man das Wesentliche, den Ausdruck und das Gefühl, das jede Person ausmacht, unabhängig davon, wie verschieden sie ist." Anja Pollnow, die längere Zeit den Unterricht besucht hat, meint zu den Fotos, die sie während ihrer Teilnahme am Tanztraining gemacht hat: „Es ist der Mut, Schönheit, Poesie und Sinn im Andersartigen, Nicht-Gewohnten zu entdecken, die uns als Menschen frei macht." Die Zitate und die Fotos finden sich auf der Website der Initiative http://www.tanzfaehig.com/tanzprojekte.html [16.07.2020]. 
ders-Sein in den Körper eingeschrieben. Vielfach müssen sie im Unterricht erst wiederentdecken, dass ihr Körper nicht nur ein Objekt der medizinischen Behandlung und der Neugierde ist oder als minderwertig und hässlich abgewertet werden muss, sondern dass auch ihr Körper gleich einem nichtbehinderten sprechen kann und es wert ist, gehört und gesehen zu werden. Dann vermag die inklusive Tanzpraxis behinderte Körper tatsächlich aus ihrer Unterordnung zu befreien (vgl. Turinsky, 2010, S. 54). Doch bisweilen sitzen die Vorerfahrungen so tief, dass sie auch das Lernen beeinträchtigen. Dann wird sogar ein auf Wunsch des Teilnehmers vorgebrachter kritischer Kommentar zu seinem Tanz zu einer erneuten Kränkung, die ihn den ganzen Unterricht entwerten lässt. Dann wird den Leitern von einer Teilnehmerin Diskriminierung vorgeworfen, wo sie eine Übung vorgeschlagen haben, die sie psychisch an ihre Grenze gebracht hat, aber körperlich ohne Weiteres hätte ausführen können.

Wie die Einstellung von Behinderten zum Tanz nicht ohne Widerspruch ist, so ist auch die Haltung gegenüber behinderten Menschen im Tanz zwiespältig. Behinderte, die tanzen, verkörpern nicht nur das Andere, sie sind es auch und ängstigen entsprechend (vgl. Weber, 2007). Ihre Anwesenheit wirft unweigerlich die Frage auf, ob man drinnen oder draußen ist, ob man gesellschaftlich am Rande oder in der Mitte steht, ob über einen verfügt wird oder man selbst die Macht hat (vgl. Titchkosky, 2005, S. 225). Vermehrt werden behinderte Tänzerinnen und Tänzer aber auch von einem Kunstbetrieb gesucht, der das Authentische vermarktet. Mit ihrer Körperlichkeit scheinen gerade Behinderte glaubhaft das verlangte Wir sind nicht zugerichtet! vertreten zu können. Angebote, die Menschen mit Behinderungen in den Tanz einbeziehen und öffentlich gefördert werden, heben demnach oft hervor, dass sie Behinderten eine berufliche Laufbahn eröffnen wollen.

\subsection{Voraussetzungen für den Unterricht}

Solange Menschen mit Behinderungen sich den vorhandenen Bedingungen anpassen und unauffällig einfügen, können sie problemlos an jedem Unterrichtsangebot teilnehmen bzw. sind sie dazu zugelassen. Schwierig wird es dann, wenn sie für ihre Teilnahme etwas brauchen, was nicht vorgesehen ist, oder wenn ihre Körperlichkeit sich nachteilig für die anderen Teilnehmer ${ }^{\star}$ innen auswirkt oder den Leiter ( ${ }^{*}$ inne)n zu schaffen macht. Dann tun sich für sie die Barrieren auf, die ihnen auch im Alltag einen Zugang verwehren. Um behinderte Tänzerinnen und Tänzer im Studio willkommen heißen zu können, sind für einen inklusiven Unterricht diese Barrieren zu erkennen und möglichst zu verringern: 
- Es betrifft als Erstes die architektonischen Barrieren. Das verlangt nicht nur ein Tanzstudio, das rollstuhlgerecht ist und in dessen Nähe sich auch eine entsprechende Toilette befindet. Dazu gehören auch die erwähnten Behindertenparkplätze vor dem Haus, ein Leitsystem für Blinde, eine gute Raumakustik für Hörgeschädigte und vieles andere mehr, damit behinderte Menschen sich ohne Einschränkungen in einem Gebäude aufhalten können. ${ }^{24}$ Doch ehe ein Angebot nicht stattfinden kann, nehmen wir beispielsweise auch ein Studio, das nicht für Rollstuhlfahrer*innen zugänglich ist, falls sich der Unterricht gezielt an Hör- oder Sehbehinderte wendet.

- Als Zweites sind die institutionellen Barrieren zu nennen. Die Zwänge, denen eine Institution in Form von Aufnahmebedingungen, Noten und Wettbewerben unterworfen ist, können oft den besonderen Bedürfnissen entgegenstehen, die Menschen mit Behinderungen haben und die sich aus der körperlichen Schädigung ergeben. Damit ein Tanzangebot inklusiv werden kann, geht es nicht darum, von vorne herein fertige Lösungen vorzuweisen, die allen behinderten Menschen gerecht werden. Es macht mehr Sinn, klein anzufangen, dabei aufkommende Schwierigkeiten zu beheben und dann den nächsten Schritt zu tun. Es hilft, wenn diejenigen, die in der Institution tätig sind, sich als Teil einer lernenden Organisation begreifen und bereit sind, gemeinsam mit den Betroffenen Wege zu finden, weil diese in der Regel am besten wissen, was sie brauchen, um teilnehmen zu können.

- Als Drittes ist an kommunikative Barrieren zu denken. Wir wählen nur solche Übungen aus, von denen wir annehmen, dass sie von den Anwesenden mit ihren körperlichen Gegebenheiten und tänzerischen Erfahrungen auch umgesetzt werden können. Das schließt Herausforderndes überhaupt nicht aus. Da auch Sprache ausgrenzen kann, achten wir auf die Worte und Wendungen, mit denen wir anleiten. Eine Aufforderung wie „Nun gehen alle durch den Raum“ grenzt eine Person aus, die einen Rollstuhl benötigt, bzw. verlangt von ihr ein eigenes Nachdenken, wie sie der Aufforderung folgen kann, während ein „Alle bewegen sich durch den Raum“ zwischen ihr und den anderen keinen Unterschied aufkommen lässt. Während wir bei Lernbehinderten versuchen, die Sprache an sich einfach zu halten, vermitteln wir blinden Teilnehmer( ${ }^{*}$ inne)n die Übungen gerne unmittelbar an ihrem Körper. Sie hören dann nicht nur die Beschreibung dessen, was zu tun ist, sondern spüren es unmittelbar körperlich, so dass sie sich nicht selbst vorstellen müssen, was wir gemeint haben könnten. Umgekehrt ist auch wichtig, darauf zu achten, dass alle verstehen, was sprachbehinderte Teilnehme-

24 In Deutschland sind die Grundlagen für die Barrierefreiheit öffentlich zugänglicher Gebäunde nach DIN 18040-1 geregelt. Sie finden sich u.a in https://nullbarriere.de/ din18040-1.htm [07.12.2019]. 
rinnen und Teilnehmer zum Tanz mitteilen wollen, auch wenn es mühsam sein mag, so oft nachzufragen, bis man sie wirklich verstanden hat. Für einen Unterricht, der Menschen mit Behinderungen einbeziehen will, erscheint es uns unabdingbar, die Zeit aufzubringen, die eine Kommunikation immer braucht, wenn unterschiedliche Menschen zusammenkommen und sich verständigen müssen.

- Als Viertes gilt es, die mentalen Barrieren zu erkennen und sich einzugestehen. Wenn man als Leiterin oder Leiter eines inklusiven Tanzangebots nicht gewohnt ist, Behinderten mit ihren geschädigten Körpern zu begegnen, kann es Angst machen. Ebenso kann es ängstigen, nicht mehr auf die gewohnte Unterrichtsweise zurückgreifen zu können und im Voraus nicht zu wissen, wie Inhalte behinderten Menschen gegenüber richtig vermittelt werden können. Hier kann es hilfreich sein, sich kundig zu machen, was ihr Leben ausmacht, sich immer wieder mit erfahreneren Pädagog $\left({ }^{*}\right.$ in$n)$ en auszutauschen und in einer Supervision das eigene Vorgehen zu reflektieren (vgl. Whatley, 2008). Ebenso gehört es zu den Barrieren, die von der Leitung eines inklusiven Tanzangebots zu überwinden sind, genau wahrzunehmen, was die Teilnehmenden empfinden. Das kann die Angst der Nicht-Behinderten vor einem Tanz mit Behinderten ebenso sein wie ihre Schaulust am abweichenden Körper, aber auch die Scheu von Behinderten, mit ihrer Körperlichkeit sichtbar zu werden oder ihre Eifersucht auf die scheinbar perfekten Körper der Nicht-Behinderten.

Trotz allen Bemühens werden sich auch in einem inklusiven Unterricht immer wieder Ausschlüsse finden, denn sie lassen sich im Umgang mit Anderen kaum vermeiden. Doch macht es einen Unterschied, ob es denen überlassen wird, sie zu überwinden, die sie wahrnehmen und darunter leiden, oder ob diejenigen, die ein Angebot leiten, sich dafür verantwortlich fühlen und bereit sind, nach Lösungen zu suchen, die alle einbeziehen. Wenn es möglich ist, die verschiedenen Barrieren zu überwinden und offen zu sein für die ungekannte Körperlichkeit der Anderen und das Unvermutete im eigenen Körper, entsteht beispielsweise ein nie gesehener Tanz von vier E-Rolli-Fahrer( ${ }^{*}$ inne)n, der wundersam dem Kurven der Auto-Scooter auf dem Rummelplatz gleicht und die Tanzenden selbst befremdet, so ungewohnt ist er. 


\subsection{Arbeitsweisen eines Tanzes in Vielfalt}

Bei einem Unterricht, an dem Menschen mit Behinderungen teilnehmen, braucht es keine speziell inklusive, sondern eine allgemein gute Tanzdidaktik und -methodik (vgl. Dinold, 2012, S. 173-175). Er beinhaltet demnach u.a. ein prozessorientiertes Vorgehen, eine Ausrichtung auf den Einzelnen mit seinen Fertigkeiten, eine angemessene Berücksichtigung des subjektiven Empfindens, die Anwendung von klaren Prinzipien und offenen Methoden sowie eine Förderung der Kreativität bei den Tanzenden (vgl. Smith-Autard, 2002, S. 12). Wer ein inklusives Tanzangebot anleiten will, sollte in der Lage sein, wertschätzend, begeisternd und einfühlsam zu unterrichten. Er oder sie muss wissen, wo seine Schüler*innen abzuholen und wie sehr sie zu fordern sind. Denn auch Behinderten kann es im Tanz darum gehen, über ihre Grenzen hinaus geführt zu werden. Oft kann ein inklusives Angebot aber eine größere Flexibilität von den Unterrichtenden erfordern als sonst. Da die Verschiedenheit der Körper im Studio oft im Voraus nicht abzuschätzen ist, kann es kurzfristig notwendig werden, einen vorgesehenen Unterrichtsplan abzuändern und ein anderes Vorgehen zu wählen. Aus der Verschiedenheit der Körper kann sich weiterhin ergeben, dass bei manchen Inhalten sich die einen überfordert fühlen und die anderen langweilen. Das muss nicht darin begründet sein, dass die einen behindert sind und die anderen nicht, denn auch in einem Unterricht, an dem nur Menschen mit Behinderungen teilnehmen, kann sich eine große Bandbreite unterschiedlicher Vorerfahrungen finden. Die Gruppe dann zu teilen und nach ihren Fähigkeiten getrennt zu unterrichten widerspricht unseres Erachtens zum einen dem Gedanken einer Inklusion, zum anderen verhindert es die besondere Gelegenheit, den Tanz aus der Vielfalt heraus entstehen $\mathrm{zu}$ lassen.

Für unseren Unterricht wählen wir bewusst Formen des Tanzes, die nicht auf Virtuosität beruhen. Unseres Erachtens bietet sich für einen Unterricht in körperlicher Vielfalt besonders ein Tanzen an, das auf der Improvisation beruht. Denn mit der Freiheit von vorgegebenen Schritten macht sie den Tanz unterschiedlichen Körpern in gleichem Maße zugänglich (vgl. Benjamin, 2002, S. 7). Rollen und Sehen, Gehen und Fliegen, Hinken und Fühlen sind dabei gleichwertig. Die Vielfalt der Bewegungsweisen, die die Teilnehmer*innen in ihrer Körperlichkeit mit sich bringen, fügt sich in einer Improvisation, bei der nicht bewertet wird, ob eine Bewegung richtig oder falsch ist, zu einem Tanz, an dem alle teilhaben können und der alle fordert, unabhängig von ihren körperlichen Fähigkeiten und Einschränkungen. Dabei heißt Improvisation nicht, dass alles möglich ist. Vielmehr sehen wir mit Adam Benjamin, dem Mitbegründer der integrativen Londoner Tanzkompagnie Candoco, die Improvisation als die Kunst, im gegebenen Augenblick das Richtige zu tun, nämlich 
das, was die Spannung im Raum, die Aufmerksamkeit der Beteiligten oder die Wahrhaftigkeit der Bewegung vergrößert; oder mit dem australischen Performer Andrew Morrish als die Kunst, in jedem Augenblick das herauszufinden und das zu tun, was einem das größte Vergnügen bereitet. Über das unvorhergesehene Bewegen erweitern wir die Improvisation in Abhängigkeit von den Anwesenden auch um ein Tönen mit der Stimme, um ein Musizieren, um ein Malen oder um ein Spielen mit den Objekten im Studio. Auch das Unterrichten wird uns zum Improvisieren. Aus dem einen Augenblick ergibt sich die nächste Übung, ohne dass wir im Voraus wissen, wohin uns gerade diese Klasse führen wird. Üblicherweise beginnen wir mit einem Aufwärmen, bei dem bei den Tanzenden zuerst der Bezug zum eigenen Körper vertieft wird, dann der zum Raum und zur Zeit und schließlich der zu den anderen; wir fahren mit Übungen fort, mit denen wir gezielt tänzerische Fähigkeiten stärken oder entwickeln können, und schließen mit einer Improvisation, bei der es mehr um die Komposition geht.

\subsection{Inhalte}

Wenn Tanzangebote geöffnet und behinderte Menschen einbezogen werden, dann ergibt sich die Frage, was geschieht, wenn Menschen, die sozial dadurch bestimmt sind, dass sie mit ihrer abweichenden Körperlichkeit gleichgesetzt werden, sich dem Tanz als einer Kunstform zuwenden, die gleichermaßen körperlich, intuitiv, emotional und nichtintellektuell ist (vgl. Cooper Albright, 1997, S. 7). Es zeichnen sich zwei mögliche Antworten ab. In dem einen Fall zielt der Unterricht darauf ab, Behinderten möglichst viele der Fertigkeiten zu vermitteln, über die Tänzer*innen mit einem normalen Körper selbstverständlich verfügen. Es geht dabei also um eine Normalisierung des behinderten Körpers und um seine Einbeziehung in bestehende Formen des Tanzes. In dem anderen Fall zielt der Unterricht darauf ab, Menschen mit Behinderungen in ihren Fähigkeiten zu stärken, so dass sie sich mit ihren körperlichen Eigenheiten den Tanz schaffen können, der zu ihrem Körper passt. Dabei geht es also um eine Veränderung bestehender Formen des Tanzes und um eine Enthinderung des behinderten Körpers.

In einer Ästhetik der Differenz (vgl. Walser-Wohlfarter \& Richarz, 2018a) begreifen wir die Bedingungen körperlicher Verschiedenheit als längst noch nicht vollends erkundete Möglichkeit, den Tanz zu gestalten. Angesichts einer sich vielfach wandelnden Gesellschaft wollen wir im künstlerischen Tanz Diversität repräsentieren und Modelle sozialer Kohärenz sinnlich erfahrbar machen. Die verschiedenen Möglichkeiten und Grenzen eines Körpers sind für uns dabei in Hinblick auf den Tanz gleichwertig. In unserem Unterricht wollen 
wir denen, die bei uns tanzen, vermitteln, was es braucht, um nach dieser Auffassung tanzfähig zu sein. Es beinhaltet für uns dreierlei:

- Da Tanz sich als Bewegung von Körpern in Raum und Zeit verstehen lässt, braucht es unseres Erachtens als Erstes die Fähigkeit, sich als Tänzerin oder Tänzer mit Absicht, Bewusstheit und emotionaler Beteiligung in Raum und Zeit bewegen zu können. Weil diese Fähigkeit nicht darauf beruht, dass ein Körper in einer bestimmten Weise auszusehen und sich zu bewegen hat, ist es eine Aufgabe, die mit jeder Körperlichkeit erfüllt, die aber auch so vielschichtig ist, dass sie vollends von keiner Körperlichkeit erschöpft werden kann. Dabei halten wir es für wesentlich, dass Tänzer*innen in ihrem Bewegen ehrlich sind und sich zu erkennen geben. Wenn sie sich zu dem öffnen, was sie in ihrem Wesen ausmacht, kann im Tanz miteinander, aber auch in einer späteren Beziehung zu einem Publikum etwas entstehen, das berührt und verbindet.

- Als Zweites braucht es die Fähigkeit, sich im Tanz verletzbar machen zu können. Das bedeutet für uns, dass Tanzende sich nicht hinter ihrer Tanztechnik verbergen, sondern sich mit ihr persönlich ausdrücken. Es bedeutet, beim Bewegen ganz in der Gegenwart zu sein und das Risiko einzugehen, zu scheitern und beschämt zu sein. Dabei ist das Zeigen verletzbarer Körper nicht vornehmlich die Aufgabe von Menschen mit Behinderung denn diese tun es sowieso (vgl. Ziemer, 2008, S. 133). Verletzlichkeit ist vielmehr ein Teil unseres Wesens, auf den wir zugreifen können, wenn wir bereit sind, uns zu öffnen, und der uns miteinander verbindet und zur Erkenntnis führt. Wenn wir unsere Verletzlichkeit zulassen, geraten wir außer uns. Insofern ist es uns wichtig, dass sich im Unterricht eine tänzerische Gemeinschaft bildet, wo die einzelnen voneinander wissen, sich schützen und vertrauen. Sie schafft die Voraussetzung für den besonderen Übergangszustand des Dazwischen, zwischen dem Innen und dem Außen, dem Eigenen und dem Fremden, der Kreativität bedeutet.

- Um in unserem Verständnis tanzfähig zu sein, braucht es daher als Drittes die Fähigkeit, kreativ zu sein. Wir folgen dabei der Annahme, dass auch im Tanz Kreativität aus Subtraktion, Variation und (De-)Formation entsteht (vgl. Ziemer, 2008, S. 41-56; Deleuze, 1980). Das heißt, dass etwas geschaffen werden kann, wenn vom Bestehenden etwas weggenommen und das, was bleibt, abgewandelt und neu gestaltet wird. Wenn wegen einer Behinderung die körperliche Leistungsfähigkeit von Tag zu Tag wechselt, ist die Vorgabe der Wiederholbarkeit einer choreografierten Bewegung aufzugeben und eine Form des Tanzes zu finden, die dieser Körperlichkeit entspricht. Daraus, dass ein Tänzer oder eine Tänzerin blind ist, muss sich nicht ergeben, dass er oder sie nicht tanzen kann. Wenn eine Form des Tanzes entwickelt wird, die nicht auf Sehen beruht, ist die körperliche Ge- 
gebenheit der blinden Tänzerin oder des blinden Tänzers nicht mehr nur kein Nachteil, sondern ist dem Körper eine Möglichkeit eingeschrieben, die sehenden Tänzer( ${ }^{*}$ inne)n fehlt. ${ }^{25}$ Indem die Tanzenden in der Choreografie zu einer stimmigen Ganzheit fügen, was bis dahin getrennt gewesen ist, können sie einen Tanz gestalten, den es so noch nicht gegeben hat und der auch ein anderes Verhältnis zwischen Publikum und Tänzer(*inne)n bedingt.

\section{Ausblick}

Tanz von Menschen mit Behinderungen nicht therapeutisch oder soziokulturell zu betreiben, sondern ihre Körperlichkeit als künstlerisch wertvolle Erweiterung des herkömmlichen Tanzkörpers zu betrachten, ist unser Anliegen mit der Initiative tanzfähig. Indem sich bei unseren Angeboten zumindest einige der sich im Tanz bewegenden Körper einer herkömmlichen Virtuosität entziehen, hinterfragen wir mit unserem Ansatz, was Tanz ist, und regen wir an, zu einer Bestimmung von Tanz zu kommen, die der heutigen Gesellschaft mehr entspricht. Dafür verwenden wir Begriffe wie Tanz in körperlicher Vielfalt oder Ästhetik der Differenz. Im Studio wie auf der Bühne haben wir den Anspruch, die Verschiedenheit der Körper sichtbar zu machen, sich von ihrer Schönheit leiten zu lassen und ihre Vielfalt zu einem stimmigen Ganzen zu gestalten (vgl. Walser-Wohlfarter \& Richarz, 2018a, S. 226). Da es sich um eine künstlerische Initiative handelt, vermitteln wir die Inhalte nicht theoretisch, sondern ästhetisch. Sinnlich wahrnehmbar wird im Tanz u.a. die Bedeutung der Kommunikation, wenn Menschen verschieden sind, das Konzept der Inklusion oder der Wert von menschlicher Verletzlichkeit für Selbsterkenntnis und Gemeinschaftsbildung. Wichtige Prinzipien im Umgang mit behinderten oder auch mit nicht-behinderten Menschen sind für uns dabei die Gleichwertigkeit des Verschiedenen und die Wertschätzung des im Augenblick Vorhandenen; eine Sichtweise des Anderen, die in ihm nicht nur das Fremde erkennt, sondern auch das Eigene; und ein Verständnis von Problemen nicht als Bedrohung, sondern als Chance für Wachstum.

Der Ansatz, wie wir ihn in der Initiative tanzfähig entwickelt haben, lässt sich nicht nur auf Diversität beziehen, die durch Behinderung bedingt ist, sondern auch auf die, die durch Alter, Herkunft, Kultur, Sexualität oder Geschlecht bedingt ist. Ebenso lässt er sich vom Tänzerischen auf das Musikalische oder andere künstlerische Medien übertragen. Wie im Tanz lassen sich

25 Über die Koproduktion eines nichtvisuellen Tanzstücks, das sehbehinderte und blinde Tänzerinnen einbezogen hat, berichten wir in Walser-Wohlfarter \& Richarz, 2018b. 
auch in der Musik verschiedene Formate des Unterrichtens verwirklichen, die vom wöchentlichen, eher offenen Angebot bis zu themen- oder behinderungsspezifischen Workshops reichen können. Auch in der Musik lässt sich eine pädagogische Intention um eine künstlerische ergänzen, lassen sich Partnerschaften mit anderen Einrichtungen aufbauen, die eine weitergehende Förderung der Teilnehmerinnen und Teilnehmer jenseits der eigenen Institution erlauben, können andere Pädagog $\left({ }^{*}\right.$ inn $)$ en weitergebildet und geeignete Dozent $\left({ }^{*}\right.$ inn)en mit Behinderung für den Unterricht gewonnen werden. Auch sind die Haltung der Unterrichtenden, die Zielgruppe eines inklusiven Unterrichts und dessen Voraussetzungen weitgehend unabhängig von den eingesetzten künstlerischen Medien. In der Musik braucht es wie im Tanz vor allem eine gute Didaktik und Methodik, wenn Menschen mit Behinderungen in den Unterricht einbezogen werden, und es lässt sich hier wie dort eine Arbeitsweise finden, die alle Beteiligten anspricht und fordert. Auch inhaltlich lässt sich in der Musikpädagogik wie in der Tanzpädagogik ein Unterricht denken, bei dem die vielfältigen Lebens- und Welterfahrungen behinderter Menschen besondere künstlerische Formen ermöglichen, die über das Gewohnte hinausgehen.

\section{Literatur}

Albert, D. (2018). Inklusive Tanzdidaktik und -methodik für den Schulsport. Wie inklusiver Tanzunterricht gelingen kann! In S. Quinten \& Ch. Rosenberg (Hrsg.), Tanz - Diversität - Inklusion. Jahrbuch TanzForschung 2018 (S. 19-30). Bielefeld: transcript. https://doi.org/10.14361/9783839443309-002

Bausch, P. (2016). Etwas finden, was keiner Frage bedarf. Rede beim „2007 Kyoto Prize Workshop in Arts and Philosophy" am 12. November 2007 in Kyoto. In S. Koldehoff \& Pina Bausch Foundation (Hrsg.), O-Ton Pina Bausch. Interviews und Reden (S. 317-322). Wädenswil: Nimbus.

Benjamin, A. (2002). Making an Entrance. Theory and Practice for Disabled and Non-Disabled Dancers. London, New York: Routledge.

Bruno, R. L. (1997). Devotees, Pretenders, \& Wannabes: Two Cases of Factitious Disability Disorder. The Journal of Sexuality \& Disability, 15, 243-260. https:// doi.org/10.1023/A:1024769330761

Cloerkes, G. (2007). Soziologie der Behinderten (3. Aufl.). Heidelberg: Winter.

Cooper Albright, A. (1997). Choreographing Difference. The Body and Identity in Contemporary Dance. Middletown: Wesleyan University Press.

Dederich, M. (2007). Körper, Kultur und Behinderung. Eine Einführung in die Disability Studies. Bielefeld: transcript. https://doi.org/10.14361/9783839406410

Deleuze, G. (1980). Ein Manifest weniger. In G. Deleuze, Kleine Schriften (S. 37-74). Berlin: Merve. 
Dinold, M. (2012). Inklusiv, kreativ und gestaltend tanzen. Pädagogische Aspekte im Sportunterricht. Sportunterricht, 61, 173-177.

Goffman, E. (1967). Stigma. Über Techniken der Bewältigung beschädigter Identität. Frankfurt a. M.: Suhrkamp.

Hermans, C. (2016). Differences in Itself: Redefining Disability through Dance. Social Inclusion, 4, 160-167. Verfügbar unter: https://www.cogitatiopress.com/ socialinclusion/article/view/699 [16.07.2020]. https://doi.org/10.17645/si.v4i4. 699

Köberl, F. (2014). Inklusiver Tanz als subversive Praxis: Eine Untersuchung zur Körperpolitik am Beispiel von Michael Turinskys heteronomous male. Diplomarbeit, Universität Wien.

Kultur für Alle e.V. (2018). Kulturbesuche barrierefrei ermöglichen. Ein Leitfaden zur Barrierefreiheit in Kultureinrichtungen. Verfügbar unter: https://kultur-fuer-alle. net/wp-content/uploads/2019/09/KFA_Leitfaden_Barrierefreiheit_Kulturein richtungen.pdf [16.07.2020].

Maskos, R. (2004). Leben mit dem Stigma. Identitätsbildung körperbehinderter Menschen als Verarbeitung von idealisierenden und entwertenden Stereotypen. Diplomarbeit, Universität Bremen, Studiengang Psychologie. Verfügbar unter: http://bidok.uibk.ac.at/library/maskos-stigma-dipl.html [16.07.2020].

McGrath, E. (2013). Beyond Integration: Reformulating Physical Disability in Dance. Dissertation, University of Bedfordshire. Verfügbar unter: http://uobrep. openrepository.com/uobrep/bitstream/10547/323750/1/eimirmcgrath.pdf [16.07.2020].

Mitchel, D. T. \& Snyder, S. L. (2000). Narrative Prosthesis. Disability and the Dependence of Discourse. Ann Arbor: University of Michigan Press.

Quinten, S. \& Schwiertz, H. (2014). Fähigkeitsgemischer Tanz - Zum aktuellen Forschungsstand. Zeitschrift für Inklusion, 4. Verfügbar unter: https://www.inklu sion-online.net/index.php/inklusion-online/article/download/254/245?inline=1 [16.07.2020].

Richarz, B. (2018a). Der biologische Körper: geschädigt. Unveröffentlichtes Manuskript.

Richarz, B. (2018b). Der soziale Körper: behindert. Unveröffentlichtes Manuskript.

Rommelspacher, B. (Hrsg.). (1999). Behindertenfeindlichkeit. Ausgrenzungen und Vereinnahmungen. Göttingen: Lamuv.

Sandahl, C. \& Auslander, Ph. (2005). Introduction. In C. Sandahl \& Ph. Auslander (Hrsg.), Bodies in Commotion. Disability and Performance (S. 1-12). Ann Arbor: University of Michigan Press. https://doi.org/10.3998/mpub.92455

Senk, K. (2015). Zur Begriffsbestimmung der Inklusion von Menschen mit Behinderung und der Frage nach Schulungsmöglichkeiten an zeitgenössischen Bühnentanzausbildungen. Der Versuch einer Analyse im deutschsprachigen Raum unter besonderer Berücksichtigung der Situation in Österreich. Bachelorarbeit, Konservatorium Wien Privatuniversität, Fakultät Darstellende Kunst. 
Siebers, T. (2009). Zerbrochene Schönheit. Essays über Kunst, Ästhetik und Behinderung. Bielefeld: transcript. https://doi.org/10.14361/9783839411322

Smith-Autard, J. (2002). Dance Composition (6. Aufl.). London: Bloomsbury.

Titchkosky, T. (2005). Looking Blind. A Revelation of Culture's Eye. In C. Sandahl $\&$ Ph. Auslander (Hrsg.), Bodies in Commotion. Disability and Performance (S. 219-229). Ann Arbor: University of Michigan Press.

Turinsky, M. (2010). Strauchelnde Gesten. Zu einer politischen Ontologie inklusiven Tanzes. gift - zeitschrift für freies theater, 2, 51-55.

Vernaldi, M. (2007). Problemzone Mensch. Mondkalb, 2. Verfügbar unter: https:// mondkalb-zeitung.de/problemzone-mensch/ [16.07.2020].

Waldschmidt, A. (2005). Disability Studies: Individuelles, soziales und/oder kulturelles Modell von Behinderung? Verfügbar unter: http://bidok.uibk.ac.at/library/ waldschmidt-modell.html [16.07.2020].

Walser-Wohlfarter, E. \& Richarz, B. (2018a). tanzfähig - Unterwegs zu einer Ästhetik der Differenz. In S. Quinten \& Ch. Rosenberg (Hrsg.), Tanz - Diversität Inklusion. Jahrbuch TanzForschung 2018 (S. 219-233). Bielefeld: transcript. https://doi.org/10.14361/9783839443309-017

Walser-Wohlfarter, E. \& Richarz, B. (2018b). Kein Tanz nur für Blinde. Über die Co-Produktion eines nicht-visuellen Bühnenstücks. Orff Schulwerk Informationen, 99, 34-37.

Weber, L. (2007). Künstler mit Behinderung - Gleichheit und/oder Differenz. Vortrag gehalten auf „Brückenschlag zwischen Behinderung und Bühne“, Symposium, 5. Juni 2007, Kultur- und Begegnungszentrum Union, Basel. Verfügbar unter: https://www.integrart.ch/assets/media/files/07_lilo-weber.pdf [16.07.2020].

Wendell, S. (1996). The Rejected Body. Feminist Philosophical Reflections on Disability. New York: Routledge.

Whatley, S. (2008). Moving Matters: Supporting Disabled Dance Students in Higher Education. Coventry: CeMAP. Verfügbar unter: http://www.invisibledifference. org.uk/media/papers/movingmatters_1.pdf [16.07.2020].

Willemsen, R. (2014). Vorwort. In R. Aguayo-Krauthausen, Dachdecker wollte ich eh nicht werden. Das Leben aus der Rollstuhlperspektive (S. 9-17). Reinbek: Rowohlt.

Williams, G. (2014). Disability, the Dancer and the Dance with Specific Reference to Three Choreographers: Caroline Bowditch, Marc Brew and Claire Cunningham. Unveröffentlichte Dissertation, University of Coventry. Verfügbar unter: https://curve.coventry.ac.uk/open/file/49662a71-d15a-48a7-a817-7bae88d03 a9b/1/Williams\%202014.pdf [16.07.2020].

Ziemer, G. (2008). Verletzbare Orte. Entwurf einer praktischen Ästhetik. Zürich, Berlin: Diaphanes. 
Beate Hennenberg \& Hana Zanin

\section{Bewusstseinswandel durch tanzkünstlerische Aktivitäten des inklusiv arbeitenden Tanzvereins Ich bin O.K.}

\section{Der inklusive Tanzverein Ich bin O.K.}

Der inklusive Tanzverein Ich bin O.K. ${ }^{1}$, der auch Kultur- und Bildungsarbeit betreibt, wurde 1979 von Katalin Zanin gegründet. Damals war es unüblich, dass sich Menschen mit Behinderung in der öffentlichen Kunstszene präsentierten. Noch bevor drei Jahrzehnte später die UN-Behindertenrechtskonvention die Teilhabe für Menschen mit Behinderung an Kunst und Kultur explizit einforderte, schuf Katalin Zanin einen Raum, in dem die gleiche Würde, die gleichen Chancen und die Anerkennung des Ausdruckspotenzials aller Menschen zum Grundprinzip erhoben wurden. Es gelangen nicht nur internationale Auftritte wie beispielsweise 1984 anlässlich des Besonderen Internationalen Kunstfestes im Weißen Haus in Washington, zu dem Jane Smith, Gründerin des amerikanischen Vereins Very Special Arts, einlud, oder 1993 bei einem Tanzworkshop in Neu-Delhi, sondern es kam auch zu einer Zusammenarbeit mit der Ballettschule der Wiener Staatsoper. Diese Zusammenarbeit führte 2001 zur Teilnahme von Tänzer( ${ }^{*}$ inne)n des Vereins an der Eröffnung des Wiener Opernballs.

Der Verein lebt bis heute das Selbstverständnis, dass jede Person Fähigkeiten besitzt, die sich bei entsprechender Ausrichtung des Unterrichts und Einstellung der Lehrenden in der Gemeinschaft entfalten können, und dass Bewegung, Tanz, körperlicher Ausdruck sowie Schauspiel als Mittel bestens geeignet sind, die individuelle Entwicklung zu unterstützen (vgl. Quinten \& Schwiertz, $2014)^{2}$. Dies geschieht auf unterschiedlichen didaktischen, sozialen und gruppenbezogenen Ebenen, aber im Wesentlichen mit demselben Ansatz: Die Bewegung und das tänzerische Potenzial der einzelnen Tänzer*innen soll sich in den Gruppen und Choreografien individuell entfalten und dabei in der Gemeinschaft wirksam werden.

1 Für weitere Informationen zum Tanzverein siehe https://ichbinok.at [16.07.2020].

2 Folgende Quelle ist abrufbar unter: www.inklusion-online.net/index.php/inklusiononline/article/view/254 [16.07.2020]. 


\subsection{Vom Tanzstudio auf die Bühne}

Im Jahr 2009 übernahmen Attila Zanin und Hana Zanin-Pauknerová den Verein als künstlerisch und organisatorisch Leitende. Ihr Schwerpunkt liegt verstärkt auf künstlerisch-tänzerischem Training im Sinne einer nachhaltigen tänzerischen Bildung. Es werden keine Vorerfahrungen erwartet, jedoch sind wichtige Kriterien, dass die Tänzer*innen selbst tanzen wollen und dass sie - bisweilen mit Unterstützung - in einer Gruppe mitmachen können. Alle Tänzer*innen gewinnen ihre ersten Erfahrungen in einer oder mehreren Studiogruppen. Dabei stellt sich heraus, welche Tanzrichtung die jungen Leute interessiert und auf welchem Gebiet sie sich entwickeln wollen. Manche tendieren zu Modern Dance, andere eher zu Hip-Hop, Musical- oder Breakdance oder zum Kreativ-Tanz. ${ }^{3}$ Bezüglich der Differenzierung nach Tanzstilen stellt der Verein für die erfahreneren Tänzer*innen, welche bereits länger Unterricht nehmen, unterschiedliche Gruppen zur Auswahl. Jede der Gruppen erarbeitet ihr eigenes Repertoire, mit dem sie Auftritte bestreitet. In jedem zweiten Jahr gestaltet das Tanzstudio mit seinen 110 behinderten und weiteren rund 30 bis 40 nicht behinderten Tänzer ( ${ }^{*}$ inne)n, darunter Studierende unterschiedlicher Ausbildungen der Musik und Kunst Privatuniversität der Stadt Wien, Studierende der Behinderten- oder Sozialarbeit der Universität Wien, die ein Praktikum im Verein absolvieren und Schüler*innen benachbarter Kooperationsschulen eine Gesamtproduktion, in der alle Gruppen an einem Thema arbeiten. Als Ausdrucksmittel werden neben dem Tanz auch Gesang, Schauspiel, Livemusik und Videokunst eingesetzt. ${ }^{4}$

\subsection{Tanzdidaktik in der Vereinsarbeit}

$J e^{\star}{ }^{\star} r$ Teilnehmende soll die Möglichkeit haben, sich gemäß den eigenen Fähigkeiten so $\mathrm{zu}$ entwickeln, dass zunehmend die einzelnen Grundübungen und Tanzschritte selbständig beherrscht werden und daraus ein eigenes Bewegungspotenzial entwickelt werden kann. Darunter natürlich auch die Tänzer*innen im Rollstuhl. Diese trainieren fast ausschließlich in den ModernDance-Gruppen oder im Ausdruckstanz. Die Trainer*innen arbeiten dahingehend, dass Tänzer*innen Bewegungselemente der Choreografie mit dem ihnen zur Verfügung stehenden Bewegungsrepertoire ausdrücken, indem sie im

3 Hier finden sich die Detailbeschreibungen der angebotenen Kurse: https://www. ichbinok.at/tanzstudio/kursbeschreibungen/ [16.07.2020].

42010 gab es die Aufführung Das Tanzende Haus, 2011 das Stück Welt/er/schöpfung, 2012 den Zauberer von O.K., 2013 die Ost Side Story, 2015 Aladins Erkenntnis und 2017 das Werk Übern Zaun schau'n. 
Laufe der choreografischen Arbeit eine eigene Rolle entwickeln, manchmal sogar eine tragende Solorolle. Die Tänzer*innen im Rollstuhl arbeiten mit Partner( ${ }^{*}$ inne)n, die quasi die Bewegungen der Gruppe als eine Person darstellen. So gibt es zahlreiche Möglichkeiten des Eingebundenseins, des Koordinationstrainings, des Einbringens der eigenen Bewegungskreativität in die Gruppe. Nicht zuletzt lernen die Tänzer*innen der Gruppe, sich mit dem Bewegungsradius und den unterschiedlichen Tempi, die das Bewegen des Rollstuhls erfordert, zu arrangieren und diese Faktoren zu integrieren.

Dabei werden die physische Leistungsfähigkeit, die Beweglichkeit, die Konzentration und die Ausdauer gefördert. Der didaktische Ansatz geht immer vom kleinsten gemeinsamen Nenner aus, nämlich jener Bewegungssequenz, die für alle Mitglieder der Gruppe ausführbar ist und woraus sich weitere Elemente entwickeln lassen. Diese Binnendifferenzierung der Inhalte, angepasst an die unterschiedlichen Fähigkeiten, ist eine ständige Herausforderung für die Tanzpädagog( ${ }^{*}$ inn)en, deren kreative und reflektierende Haltung hierbei gefordert ist, da es keine allgemein gültigen Pauschalrezepte gibt. Eine feste Rahmenstruktur und Vorplanung des Stundenaufbaus geben den Tänzer $\left({ }^{*}\right.$ inne)n Orientierung und Sicherheit. Natürlich werden geplante Verläufe auch situativ adaptiert.

\subsection{Dance Company - ein Angebot für Fortgeschrittene}

Vor zehn Jahren entstand sowohl auf Seiten der Tänzer*innen als auch bei der künstlerischen Leitung der Wunsch, für fortgeschrittene Mitglieder ein anspruchsvolleres Setting in Zusammenarbeit mit professionellen Tänzer ${ }^{*}$ inne)n, Choreograf( ${ }^{*}$ inn $)$ en und Regisseur ${ }^{*}$ inn)en zu entwickeln. Das erweiterte Angebot erfordert einen größeren zeitlichen Einsatz aufgrund zahlreicherer Trainingsstunden, Freistellungen von Arbeit und Werkstätte sowie eine konsequentere Trainingsarbeit, welche die körperlichen, darstellerischen und individuellen Fähigkeiten vertieft und weiterentwickelt. Den zunehmenden Ansprüchen in der inklusiven internationalen Tanzszene galt es zu entsprechen, was dem Verein offensichtlich gelang: Gemeinsam mit Balletttänzer $\left({ }^{*}\right.$ inne)n der Wiener Staatsoper eröffneten die Tänzer*innen der Dance Company 2017 die Special Olympics World Winter Games in Schladming. Im Jahr darauf debütierte das erste Tanzpaar mit Trisomie 21 am Wiener Opernball. Außerdem nahmen Tanzkünstler*innen 2016 erfolgreich am internationalen Wettbewerb VIBE $^{5}$ in Wien, am Tanzwettbewerb in Moskau und 2018 an den nationalen Special Olympics in der Disziplin Tanz teil.

5 VIBE ist der in Wien stattfindende internationale Wettbewerb Vienna Ballet Experience. 


\subsection{Dance Assist}

Durch Unterstützung der Servicestelle des Sozialministeriums in Wien eröffnete sich eine Option für fortgeschrittene Tänzer*innen in Richtung eines beruflichen Engagements. Zehn Personen konnten zu Dance-Assist-Tänzer ${ }^{*}$ inne)n ausgebildet werden. In zehn Wochenstunden (zusätzlich zur Arbeit und zu Verpflichtungen als Companytänzer*innen) erwarben sie zum einen Kenntnisse über Grundelemente des Modern-, Hip-Hop- und Break-Dance, zudem theoretische Grundkenntnisse, die sich zum einen auf elementares Wissen über den Körper, die Gesundheit und Ernährung beziehen, welche für das Tanzen relevant sind, sowie zum anderen Kenntnisse über Theorie und Sprache - wie etwa die Vermittlung von Anweisungen, das Einbringen von Korrekturen, oder auch das Einzählen zu Beginn einer Übung. Mit diesem Pilotprojekt leistet der Verein einen wichtigen Beitrag zur UN-Behindertenrechtskonvention im Kunstsektor. Mehrere Tänzerinnen haben inzwischen eine Anstellung von jeweils fünf Wochenstunden.

\section{Die Untersuchung}

Die im Folgenden vorgestellte Studie untersucht, ob und inwieweit die tanzkünstlerischen Produktionsprozesse des in Wien ansässigen inklusiven Tanzvereins Ich bin O.K. zu einem Bewusstseins- und Einstellungswandel gegenüber Menschen mit Behinderung beitragen können. Es wurden sowohl aktive Tänzer*innen der Dance Company wie auch deren Betreuende, Eltern, sowie drei Pädagog $\left({ }^{*}\right.$ inn)en, darunter die beiden Leitenden des Vereins, Hana und Attila Zanin, befragt. Es wurden Aussagen erwartet bezüglich Chancen, Grenzen oder Widersprüche aufgrund der in Proben und Auftritten erlebten Erfahrungen aller Beteiligten.

\subsection{Vorgehensweise}

Mithilfe narrativer Interviews, die ohne Gesprächsleitfaden mit nur einer Eingangsfrage durchgeführt werden, können sich die Befragten frei und nach ihrem Ermessen äußern. Nach Fritz Schütze (1983) ermöglicht diese völlig freie Stehgreiferzählung nach dem Stimulus in Form der Forschungsfrage, die eigenen Perspektiven des Befragten in von ihm konstruierten Sinnzusammenhängen zu erfassen: Der oder die Befragte kann sich in eigenem Ermessen äußern, die Person erzählt so lang, bis sie die Erzählung selbst beendet. Pausen 
müssen vom/von der Interviewenden ausgehalten werden. Eine Nachfragephase bei Unklarheiten ist erlaubt (vgl. Schütze, 1983, S. 284). Die Frage lautete: „Inwieweit können die tanzkünstlerischen Prozesse des Tanz- und Kulturvereins Ich bin O.K., besonders der dazu gehörenden Dance Company, zu einem Bewusstseinswandel gegenüber Menschen mit Behinderung beitragen?"

Die relevanten Kontaktdaten wurden über die Leiterin des Tanzvereins Ich bin O.K. vermittelt. Sechs Tänzer*innen der Dance Company stellten sich dem Interview, drei Mütter und drei Tanzpädagog(*inn)en. So wurden Menschen mit und ohne Behinderung befragt, teilweise wurden die Gespräche in vereinfachter Sprache geführt und die Frage adaptiert. Nach der Transkription wurden die Texte zur Autorisierung den Befragten vorgelegt. ${ }^{6}$ Die Datensammlung fand im September 2018 statt. Die Antworten waren in jedem Falle subjektiv, oft originell und lebendig. Inhaltstragende Textstellen werden dabei paraphrasiert, kodiert und danach in einem mehrstufigen Prozess in übergreifende Kategorien zusammengefasst. Dann folgten die Interpretation des Kategoriensystems und die Beantwortung der Fragestellungen mit Hilfe der Kategorien sowie eine theoretische Generalisierung.

\subsection{Bewusstseins- und Einstellungswandel (Ergebnisse)}

Folgend werden Prozesse und Ergebnisse des Bewusstseins- und Einstellungswandels bezüglich Behinderung auf gesellschaftlicher Ebene thematisiert. Zusätzlich zu größeren gesellschaftlichen Strukturen gerät auch die innerfamiliäre Struktur sowie ein Bewusstseins- und Einstellungswandel nach innen, betreffend das Selbstbewusstsein der Tänzerinnen, in den Blick.

\subsubsection{Bewusstseins- und Einstellungswandel nach außen in die gesellschaftliche Mitwelt durch Auftritte}

Alle Befragten waren der Meinung, dass es die zahlreichen professionellen künstlerischen Auftritte aller Tänzer*innen, vor allem jedoch die der Dance Company sind, die einen aktiven und bemerkbaren Bewusstseinswandel zu-

6 Deren Alter lag zwischen 24 und 74 Jahren. Insgesamt antworteten vier männliche und acht weibliche Personen. Die Namen wurden anonymisiert. Die Interviews dauerten zwischen zwanzig und neunzig Minuten und wurden bis auf zwei Ausnahmen in den Räumen des Tanzvereins im Hanuschhof hinter der Wiener Staatsoper gehalten. Die beiden anderen in einem Café in der Wiener Innenstadt und in der Kantine des Radiokulturhauses in der Argentinierstraße. Die Zitate im Text stammen aus den transkribierten Interviews der Studie. Die Interviewten wurden anonymisiert als Interviewpartner ${ }^{*}$ in 1-12 angegeben. 
gunsten einer mitmenschlicheren und Behinderung nicht ausschließenden Normalität vorantreiben. Gerade dann, wenn gesellschaftliche Themen wie Flucht und Migration auf der Bühne ausverhandelt werden wie im jüngsten Stück Über den Zaun schau'n. Über die eingangs erwähnte Eröffnung in Schladming berichtet eine Mutter: „Das war gigantisch, das geht in die Öffentlichkeit. Sie sind leistungsfähig und können was, die haben was zu bieten, was Erfrischendes.“ Ein Befragter rät, „man kann eigentlich nur mit den Auftritten und dem Rausgehen was bewirken - wo überall Menschen sind, dass man sie neugierig macht“. Ein Tänzer wünscht sogar, er könnte „mehr Auftritte machen, zeigen, was alles möglich ist“. Ein Mitarbeiter berichtet, „nach jedem Auftritt denke ich mir, jetzt müsste es doch leichter werden für unsere Leute, allein wenn den Leuten die Augen so glänzen. Da denke ich, jetzt müsste sich die Gesellschaft doch langsam öffnen." Eine Familienangehörige fasst es sachlich zusammen: „Sie können auftreten, tanzen und schauen, dass sie so viel wie möglich Menschen erreichen, positiv überzeugen, dann passiert Bewusstseinswandel.“

Inzwischen werden immer häufiger Phänomene der inklusiven Tanzszene wissenschaftlich begleitet und beforscht: Susanne Quinten setzt sich mit Mixedabled Dance unter Berücksichtigung der Lebenslage Behinderung auseinander und zeigt in ihrer Studie grundlegende Positionen zu Diversität und Inklusion im und durch Tanz, wobei Inklusion für sie ein Impuls für kreatives Schaffen darstellt (vgl. Quinten \& Schwietz, 2014) ${ }^{7}$. Shirley Salmon und Susanne Rebholz zeigen innovative kunstpädagogische Konzepte für Menschen, die von Marginalisierung betroffen sind und welche also aus eigener Kraft kaum Teilhabe- und Teilnahmemöglichkeiten am gesellschaftlichen Leben haben, als Verwirklichungsmöglichkeit (vgl. Salmon \& Rebholz, 2017, S. 83-84). Angelika Holzer (2010) verschriftlichte ihre Erfahrungen mit der Methode Dance Ability, welche Begegnungen und aktives gemeinsames Handeln im Tanz ermöglicht. Die in der wissenschaftlichen Community vernetzte Interviewpartnerin 1, zugleich Tanzpädagogin und Mutter eines Tänzers im Tanzverein, meint dazu, „es ist schon so eine breite Szene da, dass die Insider die Leistungen werten können, Maßstäbe anlegen, dass sie fachlich fundierte Kritik üben. Das ist ein Zeichen von Seriosität, das ich sehr schätze. " Für viele Beteiligte und Betroffene geht dieser Wandel nicht schnell genug, denn „wenn man im täglichen Geschäft ist, da geht einem immer alles zu langsam. Aber wenn ich mir vorstelle, wie das vor 30 Jahren war und das, was es jetzt an Möglichkeiten gibt, ist dies sicher ein Bewusstseinswandel. Wir haben es noch nicht geschafft, dass für Partizipation ein Common Sense entstanden wäre wie in an-

7 Dieser Text ist online verfügbar unter: https://www.inklusion-online.net/index.php/ inklusion-online/article/view/254 [16.07.2020]. 
deren Ländern. Wir sind noch immer die Besonderen, noch immer draußen vor.“ Jedoch: „Im Unterschied zu früher gibt es doch eine gewisse Anerkennung", so die Pädagogin.

\subsubsection{Bewusstseins- und Einstellungswandel nach außen in die gesellschaftliche Mitwelt durch neue Kunstformen}

Wie innovativ und kraftvoll fähigkeitsgemischter Tanz wirkt, erläutert eine Mitarbeiterin: „Wir bringen eben eine andere Kunstform auf die Bühne. Man muss das wie eine neue Sparte sehen. Wir wollen zu einer eigenen Ästhetik und zum eigenen Ausdruck finden, zu einer eigenen Handschrift." Wie in der Inklusionsliteratur gefordert, scheint sich hier das System an die Bedürfnisse der einzelnen Menschen anzupassen. Denn „alles ist im Wandel. Man muss sozusagen das Gewohnte immer wieder nach den Bedürfnissen umwandeln. Es entsteht ein neuer zeitgenössischer Tanz, der vielleicht nur innerhalb der Gruppe funktioniert. Der nur für diese Gruppe passt. Es ist ein Mikroklima, sehr speziell, wenn es gut sein soll. Es ist sehr anspruchsvoll für alle Beteiligten“. Interviewpartner 9 findet ebenfalls Worte: „Es geht nicht darum, etwas Bekanntes noch besser zu machen, sondern darum, dass es wirklich sehr authentisch ist. Unsere Tänzer suchen wirklich was Wahres, was Echtes. Und das berührt“.

\subsubsection{Bewusstseins- und Einstellungswandel nach außen in die gesellschaftliche Mitwelt durch das Einbinden von Künstler $\left({ }^{*}\right.$ inne)n und Politiker( ${ }^{*}$ inne)n}

Weitere Impulse hinein in die Gesellschaft versprechen sich die Befragten durch vermehrte Präsenz in Medien, durch Kooperationen mit Kunst- und Bildungseinrichtungen sowie durch das Einbinden namhafter Künstler ${ }^{\star}$ innen und Politikerinnen. Eine Tänzerin erinnert sich, wie aufgeregt das Publikum war: „In Schladming mit Helene Fischer, alle Leute aufgeregt, viele Leute kommen, - das macht sehr Spaß." Eine Mutter bemerkt dadurch eine höhere Aufmerksamkeit und „dass es etwas bringt, wenn Personen da sind, die einen Namen haben, etwa Herr Schwarzenegger. Menschen, die keine Beziehung zu Behinderten haben, die denken dann: aha! Da hören sie dann zu.“ 


\subsubsection{Bewusstseins- und Einstellungswandel aller Beteiligten nach innen - Empowerment}

Wie intensiv die Tanzaktivitäten und die fast familiäre Betreuung auf das Selbstbewusstsein der Tänzer*innen wirken, verdeutlicht die Aussage: „Ich zeig's her, weil ich’s kann.“ Auch diese zählt dazu: „Bin nicht schüchtern. Geht sehr gut. Weil ich glücklich bin, wollen Leute auch glücklich sein." Eine Tänzerin meint einfach, „ich liebe mein Tanzen“. Viele der Tänzer*innen kommen täglich, die meisten mehrfach pro Woche zum Training. Musikpädagoge Robert Wagner schrieb über dieses Selbst-Empowerment: „Viele Menschen können oft mehr, als sie sich selbst und ihre Umwelt ihnen gemeinhin zutrauen. Dieses Können gilt es, gemeinsam zu entdecken, es bewusst zu machen und daran anzuknüpfen." (Wagner, 2013) Zu den Hintergründen äußert sich eine Pädagogin: „Es ist eine schöne Gemeinschaft und es braucht eine bewusste Steuerung, dass es die schöne Gemeinschaft bleibt. Selbstverständlich ist gar nichts.“ Zur schönen Gemeinschaft erklärt eine Mutter, dass sie sich freue, „wie sie sich identifizieren mit dem Tanzverein, wie sie die Chance haben, vor Publikum zu treten, Interviews zu geben, Straßenfeste zu gestalten und lernen, sich im öffentlichen Raum zu bewegen. Und somit viel für ihren Alltag lernen. Alltagsbenehmen, auch, dass man etwas nicht tut." Toleranz wird nicht nur gepredigt, sondern findet laut Aussage einer Pädagogin statt: „Dann kommt noch etwas Vereinsspezifisches hinzu, das ich sehr schätze: Dass eine riesige Toleranz gegenüber den Eigenheiten der verschiedenen Personen da ist. Der Umgang mit schwierigen Verhaltensweisen, mit anerzogenen Macken, mit Frechheiten - sie nehmen die Interventionen an und akzeptieren sie." Die Interventionen und Beratungen richten sich auch auf Körperachtsamkeit mit allem, was dazu gehört.

\subsubsection{Bewusstseinswandel im Bereich des familiären Umfelds}

Alle Familien sind komplexe Systeme. Mit einer*einem behinderten Angehörigen müssen diese Familien nicht weniger alltäglich sein als andere. Den Menschen mit Behinderung wird heute meist ein Leben in weitgehender Normalität ermöglicht. Der Preis ist jedoch oftmals hoch, denn die sich aus der Behinderung ergebenden Belastungen sind häufig erheblich und passende Rahmenbedingungen müssen in jeder schwierigen Situation neu ausverhandelt werden.

Die in der Studie befragten Mütter stellten vor allem durch die Förderung persönlichkeitsspezifischer Bereiche, wie sie der Tanzverein Ich bin O.K. anbie- 
tet, einen gravierenden Bewusstseinswandel im familiären Umfeld fest. Eine Mutter berichtet, dass „aufgrund der Zugehörigkeit zum Tanzverein Ich bin O.K. der nähere Bekannten- und Verwandtenkreis M. [Name des Kindes, welches mittanzt] ganz anders wahrgenommen haben. Nämlich nicht als das behinderte Kind, das tanzt, sondern als eine Künstlerin, die auch Down-Syndrom hat." Hinter den Wow-Effekten der Mitwelt steht ein gut durchdachtes Netz an Förderungen, Bildungsangeboten und tanzkünstlerischen Ausbildungsstufen, welches den jungen Menschen mit Behinderung zur Verfügung gestellt wird. „Das war so ein All-Inclusive-Paket, was sie im Verein lernen mussten: Sich in einer Gruppe zurecht zu finden, Empathie zu zeigen und trotzdem sich was trauen. Nach vorn gehen und was zeigen.“ Oder: „Das Nichtabheben ist ein Thema. Super, dass sie es hier in ein richtiges Maß bringen. Die Begeisterung auf der einen Seite und den Stolz, den sie haben; und trotzdem zu wissen, ich bin nicht alleine da. Das geht nur, weil wir [...] Menschen haben, die uns unterstützen, die Lehrenden. "Und weil sich die Kinder dort „geborgen fühlen. Das ist mit Sicherheit ein großes Thema. Sonst könnten sie nicht so aus sich heraus gehen." Eltern fühlen sich durch die zu den Tanzangeboten gehörende soziale Struktur des Vereins spürbar entlastet, sind froh, dass „man sich als Mutter oder Vater etwas zurücknehmen konnte“. Eine Mutter war beruhigt, dass sie Anschluss gefunden hatte an andere Familien mit einem behinderten Kind, um auch an relevante politische und unterstützungstechnische Informationen zu kommen.

Der Bewusstseinswandel im Verständnis von Behinderung und von Akzeptanz wird erschwert, wenn beispielsweise nicht auf alle in der Familie gleich gut geschaut wird. Manche Antworten aus dem Bereich Familie bargen Spannungen. Nicht selten kam das Bild einer Helikoptermutter in den Sinn, wenn etwa eine Mutter die Kompetenzen ihres tanzenden Kindes beschrieb. Für die Geschwister dieses Kindes hatte die Mutter kaum Worte: „Und auch meine zwei anderen Kinder haben immer so geträumt von einer Karriere auf der Bühne. Aber das waren so Kinderträume. Und die M., unser Kind mit DownSyndrom, ist diejenige, die ein Star ist. Sie wird von den Geschwistern als Tänzerin wahrgenommen mit allen ihren Allüren." Einer anderen Mutter war wichtig, als sie die Lebensumstände des Sohns illustrierte, zu erwähnen, er habe „eine Kreditkarte, eine Bankomatkarte. Sein [nichtbehinderter] Bruder lebt in der Wohnung über ihm, der arbeitet nicht." Wie es dem Bruder gehen wird? Die dritte Mutter stellt lapidar fest, „im Lauf der Jahre hat man gemerkt, es haben einige doch mehr Talent oder mehr Ausdauer als andere“. 


\section{Schlussfolgerung: Bewusstseinswandel als Herausforderung des 21. Jahrhunderts}

Es lässt sich nicht leugnen, dass der viel beschriebene und erwünschte Bewusstseinswandel für eine inklusive Haltung ${ }^{8}$ eine der großen Herausforderungen des 21. Jahrhunderts darstellt ${ }^{9}$. Notwendig dabei ist eine differenzierte Auseinandersetzung mit Chancen, Grenzen, Widersprüchen und realistischen und unrealistischen Zielsetzungen, damit sich das Potenzial des Wandels entfalten kann. Der Soziologe Günther Cloerkes beschreibt Aspekte, durch welche sich allgemein Einstellungen und Verhaltensweisen gegenüber Menschen mit Behinderung ändern könnten, denn noch immer ist eine gewisse Starrheit auf Seiten der Nichtbehinderten feststellbar (vgl. Cloerkes, 1985) ${ }^{10}$. Er nennt vor allem den Kontakt zu Menschen mit Behinderung und das Wissen über sie als wesentlich für Veränderungen der Einstellung und für die Reduktion von bestehenden Vorurteilen. Mehr Wissen, etwa durch Weiterbildungen am Arbeitsplatz, durch Studienveröffentlichungen oder durch Diskussionen, kann Distanz abbauen und zu einem offeneren Umgang mit Menschen mit Behinderung führen.

Dies ist ein wichtiger, aber durchaus keineswegs zwangsläufig funktionierender Faktor. Zur Entstigmatisierung von Menschen mit Behinderung trägt auch die Stärkung der Handlungskompetenz der oder des Behinderten bei, welche Normalisierung und den gemeinsamen Austausch vorantreiben. In jedem Falle müssten die betroffenen Behinderten mitarbeiten, ja, die Sache aktiv betreiben (vgl. Cloerkes, 1985). Diese von Cloerkes beschriebenen Notwendigkeiten werden durch den Tanzverein Ich bin O.K. vielfältig realisiert. Durch die zahllosen Auftritte, nicht nur an prominenten Stätten oder im Fernsehen, durch internationale Wettbewerbsteilnahmen mit Preisen, Workshops an Schulen und Shows in Theatern kommt es zu Begegnungen vielseitiger Art und $\mathrm{zu}$ positiven Überraschungseffekten. Aufgrund des selbstgewählten Bildungsauftrags des Vereins, beispielsweise durch die semiprofessionelle Berufsausbildung zum Dance Assist, wird den tanzbegeisterten Menschen eine berufliche Option eröffnet, die wiederum zum Empowerment und zur Stärkung der Menschen mit Behinderung beiträgt und eine wichtige Forderung nach gesellschaftlicher Teilhabe erfüllt.

8 Konkrete Informationen sind im Artikel 8 des Übereinkommens über die Rechte von Menschen mit Behinderungen zu finden, vgl. StF: BGBl. III Nr. 155/2008.

9 Siehe auch das Bundesgesetz über die Gleichstellung von Menschen mit Behinderungen (Bundes-Behindertengleichstellungsgesetz - BGStG), StF: BGBl. I Nr. $82 / 2005$.

10 Folgende Informationen sind online abrufbar unter: http://bidok.uibk.ac.at/library/ cloerkes-einstellung.html [16.07.2020]. 


\section{Literatur}

Bundesgesetz über die Gleichstellung von Menschen mit Behinderungen (BundesBehindertengleichstellungsgesetz - BGStG), StF: BGBl. I Nr. 82/2005.

Cloerkes, G. (1985). Einstellung und Verhalten gegenüber Behinderten. Eine kritische Bestandsaufnahme internationaler Forschung. Berlin. Verfügbar unter: http://bidok.uibk.ac.at/library/cloerkes-einstellung.html [16.07.2020].

Holzer, A. (2010) Dance Ability. Methodik und Philosophie des inklusiven tanzpädagogischen Ansatzes. Diplomarbeit, Universität Mozarteum Salzburg.

Quinten, S. \& Schwiertz, H. (2014). Fähigkeitsgemischter Tanz - Der aktuelle Forschungsstand. Zeitschrift Für Inklusion, (4). Verfügbar unter: www.inklusiononline.net/index.php/inklusion-online/article/view/254 [16.07.2020].

Salmon, S. \& Rebholz, S. (2017). SPIEL_RAUM_MUSIK Improvisationswerkstatt. In Orff-Institut, Universität Mozarteum Salzburg \& Internationales Orff-Schulwerk Forum Salzburg (Hrsg.), Orff-Schulwerk Heute, 96, 83-84.

Schütze, F. (1983). Biographieforschung und narratives Interview. Neue Praxis, 13, 283-293.

Übereinkommen über die Rechte von Menschen mit Behinderungen sowie das Fakultativprotokoll zum Übereinkommen über die Rechte von Menschen mit Behinderungen, StF: BGBl. III Nr. 155/2008.

Wagner, R. (2013). Inklusion an Musikschulen. Ein Situationsbericht aus Deutschland. Bericht anlässlich des 1. integrativen Soundfestivals in Wien am 01. Juni 2012. Verfügbar unter: http://bidok.uibk.ac.at/library/wagner-musikschule.html [15.10.2020]. 

Stephanie Bangoura

\section{Gemeinschaft tanzen - Annäherungen an eine rituelle Tanzpädagogik mit Livemusik}

\section{Die Tanzstunde als Ritual}

Der vorliegende Artikel versteht sich als eine Annäherung an die Bedeutung ritueller Elemente in der zeitgenössischen Tanzpädagogik. Im Laufe der letzten zehn Jahre habe ich eine Methode entwickelt, die Grundelemente afrikanischer Tanz- und Musiktraditionen mit gestalttherapeutischen Ansätzen sowie Erkenntnissen aus Medizin und Psychologie kombiniert. ${ }^{1}$ Diese habe ich Bangoura-Methode genannt. Im Folgenden soll dargelegt werden, wie im Rahmen dieser Herangehensweise rituelle Elemente im Tanz mit live gespielter Musik fruchtbar gemacht werden können. Ziel ist es, die Sinnhaftigkeit des Tanzens, das Was-tanzen-wir, Was-bewegt-uns und die heilende Kraft des Tanzes erfahrbar zu machen.

Der Ritualbegriff ist weit: Bereits in den 1960er Jahren plädierte der schottische Ethnologe Victor Turner für eine interdisziplinäre und prozessbasierte Ritualforschung. Das religiöse Ritual beschrieb er als „,vorgeschriebenes formalisiertes Verhalten für Gelegenheiten, die noch keine Routine geworden sind und die einen Bezug zum Glauben an mystische [...] Kräfte oder Wesen haben, die als ursächlich für den erstrebten Effekt angesehen werden“ (Turner zitiert nach Bowie, 2000, S. 153). Im Unterschied zum französischen Ethnologen Émile Durkheim (1981), der dem Ritual eine systemerhaltende Funktion zuschrieb, war Turner der Meinung, dass Rituale das Potenzial besitzen, neue Ideen zu erzeugen, welche die soziale Ordnung herausfordern und damit unter Umständen zu (revolutionären) Veränderungen in Kultur, Gesellschaft und Politik führen können (vgl. Alexander, 1997, S. 140). Auch in den zeitgenössischen Kultur-, Geistes- und Sozialwissenschaften (Wulf, 2006) werden vermehrt Anstrengungen unternommen, Rituale im transkulturellen Kontext zu betrachten. Bobby C. Alexander beschreibt vor allem zwei Qualitäten: „Ein Ritual im allgemeinsten und grundlegendsten Sinn ist geplante oder improvisierte Performance, die eine Überleitung des alltäglichen Lebens in einen alternativen Zusammenhang, in dem der Alltag transformiert wird, bewirkt."

1 Wenn in der Folge von afrikanischen Tänzen die Rede ist, so beziehe ich mich vor allem auf westafrikanische Yoruba- und Mandinka-Traditionen. Diese sind mir durch meine lange Erfahrung in verschiedenen afrikanischen Ländern sowie mit Tanztheater- und Musikgruppen aus Dakar, Paris, New York, Havanna, Salvador, Hamburg und München besonders vertraut. 
(Alexander, 1997, S. 139) Basierend auf dieser allgemeinen Definition, möchte ich einige Merkmale des Rituals herausstellen, die meiner Ansicht nach für die tanzpädagogische Praxis von besonderer Relevanz sind:

- Jedem Ritual liegt eine Absicht, ein Anliegen zu Grunde.

- Rituale feiern und sichern Übergänge (z.B. Transformation von Identität, gesellschaftlicher Rollen, Status und Kompetenz).

- Symbolhafte Handlungen vermögen Unsichtbares sichtbar und fühlbar zu machen.

- Das Ritual fokussiert und kanalisiert Energie.

- Im Ritual gibt es keine Zuschauenden, nur Teilhabende.

- Das Ritual öffnet die Wahrnehmung für Unbewusstes, Magisches, Mythisches, sich zu entfalten und zu wirken.

Unabhängig von spezifischen Elementen kann jede Tanzstunde als Ritual gefeiert werden, das sich in drei Phasen - Separation, Transformation und ReIntegration in den Alltag (vgl. Van Gennep, 1986, S. 50) - beschreiben lässt. In säkularen Gesellschaften fehlen sinngebende, kollektive Rahmungen, die das Individuum in Phasen des Umbruchs begleiten und stützen könnten. Der Tanzstunde kommt daher gerade in Zeiten sozialer Atomisierung eine enorme Bedeutung zu: Der im Ritual geschaffene Raum bietet in Phasen von Schnelligkeit, Zerstreuung und Desorientierung einen wichtigen Pol der Ruhe, Konzentration und Gemeinschaftlichkeit. Für die Leitenden und Lehrenden gilt es daher, diesen Vorgang so zu unterstützen und begleiten, dass er geordnet vollzogen werden kann.

Viele, auch europäische Tanzformen, wie etwa das klassische Ballett, haben sich aus rituellen Zusammenhängen entwickelt. ${ }^{2}$ Es macht daher wenig Sinn, die äußere Form von Ritualen zu kopieren, da diese in einem singulären Kontext entstanden sind. Die Bangoura-Methode versteht sich als eine transkulturelle Herangehensweise, die nach neuen Wegen sucht, traditionelle, rituelle Tanzformen für heutige Situationen und Bedürfnisse fruchtbar zu machen. Ein Tanz kann nur dann als sinnvoll und wirksam erfahren werden, wenn er an die Bedürfnisse und die Situation derjenigen angepasst wird, die ihn tanzen. Darin manifestiert sich auch eine gestalttherapeutische Haltung: Die Wahrnehmung, Freiwilligkeit und Selbstbestimmtheit der Teilnehmenden/Tanzen-

2 In ihrer Kulturgeschichte des Balletts beschreibt Dorion Weickmann (2002) zum Beispiel, wie sich das Ballett aus den gesellschaftlichen Tänzen und Körpertechniken der europäischen Höfe entwickelte. Dies eröffnet eine neue Sichtweise auf Tanz: Dieser wird nicht als eine inszenierte Kunst oder ästhetische Form allein betrachtet, sondern als kultur- und sozialgeschichtliches Produkt, in dessen Zentrum der Leib sowie rituelle Formen kollektiver Zusammengehörigkeit und Disziplinierung stehen. 
den werden stets geachtet. Das Tanzritual lebt von den Resonanzen zwischen Tanzenden und Musizierenden - beide unterstützen, stimulieren und fordern sich untereinander und gegenseitig in einem fortwährenden Dialog. Die Basis für spontane individuelle Tanzeinlagen bilden lang ausgedehnte, wiederholte Bewegungen, Rhythmen und Gesänge der Gruppe. Aus dieser gemeinsamen Energie heraus entsteht, was ich eine tragende Gruppe nenne. Im gemeinsamen Tanzen kann die intersubjektive Erfahrung entstehen, sowohl von der Gruppe getragen zu werden, als auch selbst ein tragendes Mitglied dieser Gruppe zu sein.

Die Bangoura-Methode kombiniert dabei auf synergetische Weise afrikanische Traditionen mit zeitgenössischen Bewegungskonzepten und Trainingsformen. Diese enthalten Konzepte aus Gestalttherapie, Stimmbildung, Rhythmusschulung, Theaterpädagogik und zeitgenössischer Tanzimprovisation sowie einzelne Elemente wie Trancereisen, Selbstmassagen, Übungen aus Yoga und Pilates. ${ }^{3}$ Die leitenden Tanzpädagoginnen und Tanzpädagogen entwickeln in Resonanz mit den Tanzenden und Musizierenden eine rituelle Struktur und setzen deren Elemente situationsgerecht ein. Dabei ist es wichtig, dass die eigene Wahrnehmung auf das Hier und Jetzt ausgerichtet wird: auf die musikalischen Klänge, die Gruppe und den eigenen Körper. Dies verhindert, dass Handlungen mechanisch oder gar dogmatisch miteinander verkettet werden. Einzelne Handlungen (räuchern, Musik spielen, klatschen, tönen, rufen, singen, schreien, schütteln, lachen, weinen, tanzen, gehen) werden Teil eines kreativen, offenen Ganzen.

Das Kontaktangebot, wie es in der Gestalttherapie gelehrt wird, findet seinen Höhepunkt im spontan improvisierten Tanz, der danse individuelle: Eine Tänzerin oder ein Tänzer tanzt alleine in der Kreismitte im Dialog zur Livemusik. Musik und Präsenz der Gruppe geben Halt, Kraft und Orientierung, damit der ${ }^{\star}$ die Tanzende seinen ${ }^{\star}$ ihren Impulsen zur Selbstregulierung folgen kann. Das Gelingen erfordert von den Leitenden absolute Aufmerksamkeit für die Dynamik in der Gruppe. Es gilt das Prinzip der Partizipation, das Ritual soll gemeinsam gestaltet und nicht konsumiert werden.

Ein- und Austritt (Anfang und Ende) des Rituals stellen eine sensible Phase dar, die von den Leitenden geführt und gestaltet werden will. In diesen Übergangsphasen gilt es für Achtsamkeit und Verlangsamung zu sorgen, damit sie sicher vollzogen werden können. Die ${ }^{\star}$ Der Einzelne wird von ihremseinem gewohnten Umfeld getrennt und braucht Zeit und Vertrauen, um Teil einer neuen Gemeinschaft zu werden, bzw. um mit neuen Erfahrungen wieder zurück in das alte Umfeld zu gelangen. Wenn die oder der Tanzende aus dem

3 In meinem Buch Die Kunst, Gemeinschaft zu tanzen (in Vorbereitung) erläutere ich, wie diese Elemente in der pädagogischen Praxis zusammengeführt werden können. 
Kreis in die Mitte geht, verlässt sie oder er die Gruppe. Sie Er ist allein - in der Kreismitte zählt einzig der Mut zur Singularität, der Mut, sich von der Gruppe zu lösen. Kulturgeschichtlich betrachtet wird hier die Überwindung basaler Ängste ausagiert. ${ }^{4}$ Im Tanzritual kann die menschliche Urangst vor dem Verlassen- und Verlorensein, vor Einsamkeit, Lächerlichkeit, Schwäche und vor dem Versagen durchlebt werden. In dieser essenziellen Konfrontation liegt das Potenzial, Neues zu schaffen: neue Bewegungen und Erfahrungen, die wiederum zu Unabhängigkeit, Freiheit, Glück, Veränderung, Transformation und Heilung führen können. Das rituelle Klatschen nach dem individuellen Tanz begleitet den*die Tänzer*in zurück in die Gemeinschaft. Feedbackrunden ermöglichen es, spezielle Momente mit der Gruppe zu teilen, zu verbalisieren und den individuellen Bewusstwerdungsprozess zu unterstützen.

\section{Verkörperte Symbole}

Für Turner bildet das Symbol die kleinste Einheit des Rituals (vgl. Turner, 1967, S. 19). Eingebunden in ein größeres symbolisches Netz stiftet es bedeutungsvolles soziales Handeln. Er beschreibt Symbole als polymorphe, d.h. vielgestaltige und vieldeutige Elemente. Turner leistete mit seinen Ritualforschungen im südlichen Afrika, in Mexiko, Brasilien und Irland einen wichtigen Beitrag dazu, körperliche Symbolik als eine Basis der Organisation der sozialen Welt kenntlich zu machen.

Während meiner langjährigen Aufenthalte im Senegal war ich immer wieder von der Gleichförmigkeit der Begrüßungsrituale und davon, wie diese verkörpert werden, fasziniert. Die senegalesische Begrüßung zielt darauf, die Hierarchie zwischen den Anwesenden sowie die friedfertige und wohlgemeinte Absicht der*des zu Begrüßenden klarzustellen. Dies geschieht jedes Mal in ausführlicher, geduldiger und gleicher Weise. ${ }^{5}$ Aus europäischer Sicht ist es ein Sprechen ohne inhaltliche Relevanz, ohne Wahrheit. Doch hier geht es allein um die Haltung - eben darum, auf vorgesehene Weise $\mathrm{zu}$ antworten und so

4 „Gegen Tod und Not, den großen Eltern der Angst, wird alles aufgeboten, was die Menschen an intelligenten Vermögen und technischen Fertigkeiten zur Verfügung hatten. Alle Positivität, die Lebensfreude und ihre tausendfachen Festriten, erfordert Entlastung und Abwesenheit von Angst. Diese zu bewältigen und zu vertreiben, ist erste Aufgabe aller Kultur. Kulturelle Überschüsse, die der Steigerung von Vergnügen und Lust dienen, können erst entstehen, wenn Tod und Not, wenn Angst nicht mehr allein die Aktivitäten der Gemeinschaft bestimmt." (Böhme, 2000, S. 223)

5 Auf die Frage: „ana wa kergi?“ wird stets „nungi fa“ geantwortet. Übersetzt bedeutet dies: „Wo sind die Leute deines Zuhauses?“ „Sie sind da." Auf die Frage: „nanga def?" (Was machst du?) wird in der Regel „Ich bin hier.“ geantwortet. Ein solcher Dialog kann bis zu 15 Minuten lang fortgeführt werden. 
ein respektvolles Ambiente herzustellen. Im Gesprächsablauf werden direkte Fragen vermieden und es ist dem - meist männlichen - Ranghöheren überlassen, auf welche Weise er Themen erörtert oder sich diesen nähert. Diese Dynamik erlebte ich auch im tänzerischen Dialog mit den Musizierenden, die meine Bewegungen musikalisch umspielten, ohne sie dabei zu strikt zu führen. „Es ist alles eine Frage des Respekts“, sagte ein Musiker einmal zu mir.

Die komplexe Symbolik afrikanischer Tänze wirkt auf Außenstehende überwältigend. In meiner Arbeit habe ich mich auf Bewegungen konzentriert, die existentielle menschliche Themen bearbeiten und über den afrikanischen Kontext hinausgehend verständlich sind. Dies sind Bewegungen, die Befreiung, Aufladung und Verbindung nicht nur darstellen, sondern tatsächlich herstellen. Das energetische Bad der Orishatänze ist ein Beispiel für symbolisches Tun, das eine Realität schafft und erlebbar werden lässt. In der Bangoura-Methode wird es durch die Ausführung von Eintauch-, Abdusch-, Streich- und Schüttelbewegungen von den Musizierenden erfahrbar. Das Baden in der Musik fördert die Wahrnehmung für die live gespielte Musik und ihre Energie. Durch die Wiederholung am Ende jeder Tanzeinheit wird es zu einem ordnenden Element, das den rituellen Rahmen stützt.

\section{Der Kreis im Tanzritual}

„Warum im Kreis? Ein Kreis ist eine geniale Form. Es gibt kein Oben und Unten, kein Wichtiger und Unwichtiger, keinen Chef und Untergebene, keine Rangordnung oder Hackordnung. Alle sind gleich. Wer sich in einen Kreis begibt, ist ein Teil vom Ganzen. Das entspricht der natürlichen Ordnung auf dieser Erde, die ebenfalls rund ist und keine Rangunterschiede macht. Ein Ritual im Kreis löst Wertungen auf." (Francia, 2011, S. 8-10)

Charakteristische Bewegungs- und Aufstellungsformen in vielen traditionellen afrikanischen Tanzritualen sind Kreis, Halbkreis und Spirale. Ein Halbkreis lässt einen weiteren Halbkreis hinzudenken und auch die Spirale beschreibt in ihrem Kern einen Kreis. Im Rahmen der Bangoura-Methode ordnen sich die Musizierenden im Halbkreis an, Zuschauende und Teilnehmende bilden einen komplementierenden Halbkreis. Individuell improvisierte Tanzeinlagen werden häufig durch einen Spirallauf eingeleitet. Die Tanzenden nehmen Blickkontakt zu den Zuschauenden auf. Spiralen können auch gemeinschaftlich getanzt oder auf dem Boden mit Blättern, Sand oder Mehl gezeichnet werden. Auf symbolischer Ebene repräsentieren Kreis und Spirale ein archetypisches Muster. Sie können mit dem Kreislauf der Erde, den Zyklen von Tod und Leben, ökologischen Nahrungsketten oder Wasserkreisläufen in Verbindung gebracht werden. Der Kreis ist ein Motiv der Verwandlung - nichts bleibt wie es ist, nichts verschwindet und alles kommt in anderer Erscheinung wieder. 
In der rituellen Tanzpädagogik bedeutet der Kreis sowohl Schutz als auch Konzentration. Wer den Schritt in die Kreismitte wagt, empfängt die Aufmerksamkeit der Gruppe und steht im Spannungsfeld. Der Tanz im Kreis unterstützt idealerweise die Teilnahme, Zugehörigkeit, Wahrnehmung und Wertschätzung aller Anwesenden, ohne dass eine Person dabei bloßgestellt oder ausgestellt werden würde. Gleichzeitig ermöglicht er selbstinitiiertes Heraustreten und Sich-Zeigen. Es geht darum, sich den Raum im Mittelpunkt zu nehmen und auszudrücken, was bis jetzt zurückgehalten wurde, was (noch) nicht ausgedrückt werden durfte. Gruppe und Musizierende nehmen wahr, wo sich der*die Tänzer*in zurückhält (traumatische Spur) oder loslässt (erotische Spur). Sie spiegeln dies durch Rufe, Töne, Klatschen, Trommelschläge oder andere Gesten. Jede ${ }^{\star}$ nimmt teil, sei es durch Zuschauen, Klatschen oder Schreien - alles dient der energetischen Krafterzeugung für das Geschehen und für $j^{j e d e^{\star} n \text { Einzelne }}{ }^{\star} n$. Der Kontrast zum konventionellen Spektakel liegt auf der Hand: Es gibt keine passive anonyme Masse, die im Dunkeln unbeweglich sitzt und zuschaut. Umgekehrt sind im Ritual die Darstellenden „immer auch das Publikum ihrer selbst“ (Wulf \& Zirfas, 2004, S. 27).

In kapitalistischen und patriarchalen Gesellschaften werden fortwährend Hierarchien (re-)produziert. Im Kreis können diese Grenzziehungen erlebt, ausagiert und zugleich in die Schwebe versetzt werden. In meiner pädagogischen Praxis erfahre ich immer wieder, welch befreiende und heilende Rolle dem Kreis zukommt. Er wirkt Imitation, Vergleich, Konkurrenz, Ausgrenzung und Hierarchie entgegen und begünstigt Blickkontakt, Begegnung, Partizipation, Kooperation, Zugehörigkeit und Integration. So schreibt die Tanztherapeutin Alexa Junge: „Tanzen ist nicht nur eine gesellige und sozial gleichmachende Aktivität, sondern auch oft marginal und anormal. Es schafft einen Kontrast zum Alltag und versetzt den Tänzer in einen zeitlosen Zustand außerhalb seiner strukturierten Routine.“ (Junge, 2010, S. 6)

Die Leitenden der Bangoura-Methode achten daher darauf, einen Kreis ohne energetische Löcher zu bilden. Die Tanzenden werden angehalten, ihren Nachbarn zu spüren, Schulter an Schulter zu tanzen, die Musizierenden miteinzubeziehen, d.h. ihnen nicht den Rücken zuzuwenden. Der Kreis ist eine zentrale Form - genauso wichtig ist es aber auch, ihn wieder aufzulösen, Diagonalen und andere Möglichkeiten zu nutzen. Tanzen bedeutet, den Raum in vielfältiger Weise zu beleben und zu erfahren. 


\section{Energie und energetische Regeln}

Freundschaftliche Rituale beruhen auf Gegenseitigkeit und einer sich wechselseitig aktivierenden Energie: Ich gebe und bekomme. In jeder Kultur existieren Regeln, die - auch wenn sie den sozial Agierenden nicht bewusst sind - dabei helfen, Aufmerksamkeit und Energie zu lenken. Tanzveranstaltungen, wie ich sie im Senegal erlebt habe, seien es Konzerte, Feste, Workshops, Proben oder eine Hochzeit, sind ein gutes Beispiel, wie in einem außereuropäischen Kontext mit Geld umgegangen wird. Im Kontrast zu den oft undurchsichtig verlaufenden Transaktionen der westlichen Marktwirtschaft werden Musikerinnen und Musiker im Senegal stets in bar und vor Ort bezahlt: Sie bekommen Geldscheine vor den Augen aller zugesteckt, an die Stirn geklebt oder sie werden ihnen auf eine andere Art und Weise im Zuge eines performativen Akts gegeben. Nicht selten fliegen Banknoten durch die Luft. Geld ist Energie und seine sichtbare Übergabe bedeutet öffentliche Wertschätzung und motiviert zum Weiterspielen.

Ein anderes Phänomen ist, dass Gäste am Ende eines Konzerts oft auf die Bühne gehen, um sich dort mit improvisierten Tänzen zu zeigen. Sie genießen es, auf der Bühne neben den Stars zu stehen, Würdigung zu geben und zu bekommen. Das Heraustreten aus dem Publikum erhöht die Energie des Tanzenden sowie aller Anwesenden.

Rituelle Regeln sind nicht zu verwechseln mit Höflichkeiten. „Im Ritual kann jegliche Höflichkeit abgelegt werden. Wenn man einmal die Linie gezogen hat, die den geheiligten Raum umschließt, und die spirituellen Kräfte angerufen hat, gibt es kein Lügen, kein Heucheln." (Somé, 1999, S. 91) Es sind die Linie, der Schutzkreis, die Entscheidung und innere Ausrichtung, die ein Tanzritual entstehen lassen.

\section{Ausblick}

Aus gestalttherapeutischer Sicht ist jede Krise mit einer Chance verbunden (vgl. Hartmann-Kottek, 2011, S. 162). Krankheiten, Unwohlsein oder Unzufriedenheit stehen oft am Anfang einer Bewegung, im wortwörtlichen und im metaphorischen Sinn. Sich der eigenen Emotionen (lat. e-motus für dt. herausbewegt, erschüttert) gewahr zu werden und sie in Bewegung zu verwandeln, sich herauszubewegen, liefert gute Voraussetzungen für ein energiereiches Tanzritual. Hier treffen sich die Anliegen von Künstler(*inne)n und Therapeut $\left({ }^{*}\right.$ inn)en: Sie heißen Emotionen willkommen und transformieren sie. Es gilt herauszufinden, welche Frage oder welches Begehren im Zentrum des ge- 
meinsamen Rituals stehen soll. Emotionen nicht nur zuzulassen, sondern auch $\mathrm{zu}$ zeigen und kollektiv zu bearbeiten, setzt Vertrauen und einen sicheren Raum voraus, der nicht spontan, sondern nur über einen längeren Zeitraum hinweg entsteht. In der Bangoura-Methode arbeite ich daher bevorzugt über mehrere Jahre mit einer Kerngruppe.

Jedem Ritual liegt ein Wunsch nach Veränderung zu Grunde. Gleichzeitig ermöglicht das Ritual eine Versöhnung mit dem, was ist. Im Spannungsfeld zwischen Innehalten und Bewegung liegt sein Potenzial: Erst was akzeptiert, benannt und gesehen wurde, kann sich verändern. Dieser haltgebende Boden wird im Ritual geschaffen.

Tanzen in der Bangoura-Methode bedeutet, eine rituelle Verbindung mit sich und dem Gegenüber aufzunehmen. Das Gegenüber ist die live gespielte Musik, der Moment, die Gruppe, die Erde, der ${ }^{\star}$ die Nachbarin, die Ahninnen und Ahnen. Die Bangoura-Methode schafft rituelle Räume, um diese Prozesse sicher zu vollziehen. Tanzen wird so zu einem machtvollen Mittel der Transformation. Es ermöglicht eine tatsächliche Transformation von Selbst und Wirklichkeit: Nichts ändert sich, bis ich mich selbst verändere. Und dann ändert sich alles!

\section{Literatur}

Alexander, B. C. (1997). Ritual and current studies of ritual: Overview. In S. D. Glazier, Anthropology of Religion: A Handbook (S. 139-160). London: Greenwood Press.

Bowie, F. (2000). The Anthropology of Religion. An Introduction. Oxford: MPG Books Ltd.

Böhme, H. (2000). Leibliche und kulturelle Codierungen der Angst. In Große Gefühle. Bausteine menschlichen Verhaltens, hg. v. ZDF-Nachtstudio (S. 214-240). Frankfurt am Main.

Durkheim, É. (1981). Die elementaren Formen des religiösen Lebens. Frankfurt am Main: Suhrkamp.

Francia, L. (2011). Der magische Alltag. München: Nymphenburger.

Hartmann-Kottek, L. (2011). Gestalttherapie - heute. Psychotherapeutenjournal, 2, 162.

Junge, A. (2010). Therapeutische Wirkung von Tanz: Eine Untersuchung der Heilung im Tanz unter den Gesichtspunkten des Rituals und der Tanztherapie. Norderstedt: GRIN Verlag

Somé, S. (1999). Die Gabe des Glücks. Rituale für ein anderes Miteinander. Berlin: Orlanda Frauenverlag.

Turner, V. (1967). Forest of Symbols. Kingsport: Kingsport Press.

Van Gennep, A. (1986). Übergangsriten. Frankfurt am Main: Campus-Verlag. 
Weickmann, D. (2002). Der dressierte Leib. Kulturgeschichte des Balletts (15801870). Frankfurt am Main: Campus Verlag.

Wulf, C. (2006). Anthropologie kultureller Vielfalt. Interkulturelle Bildung in Zeiten der Globalisierung. Bielefeld: transcript. https://doi.org/10.14361/9783839405741

Wulf, C. \& Zirfas, J. (Hrsg.). (2004). Die Kultur des Rituals. München: Wilhelm Fink Verlag. 



\section{KlaWIR am Bahnhof}

\section{Das gemeinschaftsbildende Potenzial eines Community-Music- Projekts im öffentlichen Raum}

\section{Einleitung}

Können mit Hilfe eines Klaviers im öffentlichen Raum Menschen zusammengebracht werden? Wie funktioniert es, allen Menschen, egal mit welcher Vorerfahrung, Klavierspielen oder Musizieren generell zu ermöglichen?

An über hundert Bahnhöfen in Frankreich befinden sich Klaviere mit der Aufschrift À vous de jouer! (Übersetzt: Ihr seid dran!). Durch regelmäßiges Beobachten derer, die darauf spielten, die miteinander musizierten und gemeinsam zuhörten, konnte festgestellt werden, dass neben beeindruckenden musikalischen Leistungen am Klavier Situationen entstanden, in denen Menschen miteinander auf verschiedene Arten und Weisen in Kontakt kamen. Bei der genauen Betrachtung der gemeinschaftsbildenden Prozesse, die am Klavier und in dessen unmittelbarer Umgebung stattfanden, waren vor allem Menschen, die schon Klavierspielen konnten - oft sogar sehr gut -, zu beobachten.

Doch allein ein potenziell allen zugängliches Klavier führt offenbar nicht dazu, dass es auch von allen bespielt wird. Die Hemmschwelle, sich an ein Klavier zu setzen, erscheint nach wie vor groß. Viele trauen sich nicht zu spielen: weder diejenigen, die noch nie an einem Klavier saßen, noch solche, welche die Fähigkeit zu spielen hätten. Mit KlaWIR am Bahnhof war die Idee zu einem Projekt geboren, das an Bahnhöfen in Deutschland Klaviere nicht nur zur Benutzung zur Verfügung stellt. Die Initiatorin des Projekts ${ }^{1}$ entschied sich dafür, ein Begleitprogramm zu entwickeln, das Hemmschwellen abbaut und gleichzeitig das Klavier im öffentlichen Raum Bahnhof als musikalisch-sozialen Knotenpunkt der Stadtgesellschaft weiter stärkt.

In den Jahren 2017-2020 wurde das Projekt an unterschiedlichen Standorten realisiert, forschend begleitet und basierend auf den gemachten Erfahrungen kontinuierlich weiterentwickelt. Dabei wurden verschiedene Fragen thematisiert: Wie ist es möglich, Menschen zum Musizieren zu motivieren, die noch keine musikalische Vorerfahrung haben? Welche gemeinschaftsbilden-

1 Das Projekt und die Idee entstand im Rahmen der Masterarbeit von Mona Heiler. Zur Realisierung wurde die KlaWIR am Bahnhof GbR gemeinsam mit Lisa-Marie Trodtfeld gegründet. 
den Auswirkungen haben öffentlich platzierte Instrumente auf die Passantinnen und Passanten?

Ausgehend von diesen Fragen wird im Folgenden das Projekt KlaWIR am Bahnhof mit seinen unterschiedlichen Formaten vorgestellt und mit seinen spezifischen Auswirkungen analysiert.

\section{Projektbeschreibung}

Zur Orientierung der Projektentwicklung von KlaWIR am Bahnhof diente das Modell der Community Music. Die 2013 von Alicia de Banffy-Hall gegründete Münchner Community Music Aktionsforschungsgruppe (MCMAG) definiert Community Music wie folgt:

„Internationaler Fachbegriff für musikalische Aktivitäten, bei denen der kreative und soziale Prozess gleichrangig sind. Gemeinschaftliches Musizieren schafft Raum für ästhetischen Ausdruck und inklusive Praxis. Zivilgesellschaftliches Engagement wird gefördert und Menschen unterschiedlichster Herkunft zusammengebracht.“ (de Banffy-Hall, 2018, S. 103-104)

Das Konzept von KlaWIR am Bahnhof garantiert, dass während des Projektzeitraums dauerhaft ein Klavier in der Bahnhofshalle steht. Es kann jeden Tag, 24 Stunden lang, von allen Interessierten bespielt werden. Das Projekt soll jedoch nicht ausschließlich eine Möglichkeit bieten, Klavier zu spielen beziehungsweise Klavierspielen zu lernen, sondern weitläufiger verstanden werden und generell zum Musikmachen auffordern. Dafür wurden vier verschiedene Vermittlungsformate entwickelt, die es unterschiedlichen Zielgruppen, von vollständigen Anfängerinnen und Anfängern im Musikspiel, über Fortgeschrittene, bis zu professionellen Musikerinnen und Musikern ermöglichen, bei KlaWIR am Bahnhof aktiv mitzuwirken.

Das Projekt wurde, nach einigen Vorstellungs- und Kooperationsgesprächen mit der Deutschen Bahn, der Werbegemeinschaft der Deutschen Bahn AG Station\&Service (Mein Einkaufsbahnhof), Yamaha und dem Musikhaus Schulte erstmals 2017 für sechs Monate am Braunschweiger Hauptbahnhof durchgeführt. KlaWIR am Bahnhof 2018 fand neben Braunschweig zusätzlich an den Hauptbahnhöfen Hildesheim und Bremen statt. 2019 kam der Bahnhof Halle (Saale) dazu, an dem das Projekt im Rahmen und Zeitraum der HändelFestspiele stattfand.

Partizipation und Interaktion sind die Hauptprinzipien von KlaWIR am Bahnhof. Es sollte sich durch regelmäßig stattfindende Veranstaltungen auszeichnen, die im besten Fall zu autonom funktionierenden, von den Organisatorinnen und Organisatoren unabhängigen Projekten werden. Eine sich lang- 
sam entwickelnde Gruppe an Nutzerinnen und Nutzern und Zuhörenden des KlaWIRs sollte - gegenüber der sich erfahrungsgemäß schnell anhäufenden großen Traube des Publikums - gerne in Kauf genommen werden. KlaWIR am Bahnhof will Reisende und sich am Bahnhof Aufhaltende anziehen und zum Verweilen, Zuhören und Mitmachen einladen. Hierfür wurden folgende Vermittlungsformate entwickelt:

\subsection{MitMACHkonzerte}

Im Projektzeitraum finden regelmäßig MitMACHkonzerte statt, bei welchen das KlaWIR im Mittelpunkt steht. Diese werden von Bands, die von den Projektorganisatorinnen angefragt und rekrutiert werden, angeleitet. An jedem KlaWIR spielen im Projektzeitraum mindestens zwei verschiedene Bands. Es sind Konzerte, an denen alle Passantinnen und Passanten am Bahnhof durch verschiedene musikalische Mitmachaktionen teilnehmen und mitmusizieren können. So ist es möglich, zum Beispiel durch Beatboxen und einfache, eingängige chorische Phrasen zum Nachsingen, durch das Nachspielen von Rhythmen mit dort zur Verfügung gestellten Percussion-Instrumenten oder mit einem selbst mitgebrachten Instrument beim Programm und den Stücken der Bands mitzumachen. Beim Repertoire der Bands handelt es sich um eigene Stücke und teilweise auch um spontan improvisierte Musik.

\subsection{Play TWOgether}

Ein am KlaWIR angebrachter QR-Code führt zu einem Video. Die dort sichtbare, kurze Filmszene dauert 1:43 min $^{2}$ und zeigt die Abbildung von zwei Händen (Spieler*in 1 und Spieler*in 2 bzw. linke Hand und rechte Hand) auf mit Zahlen markierten Tasten.

2 Die Szene sollte möglichst kurz gehalten werden, um auch denjenigen Passantinnen und Passanten die Chance zu geben, Play TWOgether auszuprobieren, die Interesse, jedoch nicht sehr lange Zeit haben, sich am KlaWIR aufzuhalten. Außerdem wurde vermutet, dass die Ausprobierschwelle für die meisten niedriger bleibt, wenn das Video weder zu lange noch zu komplex ist. 

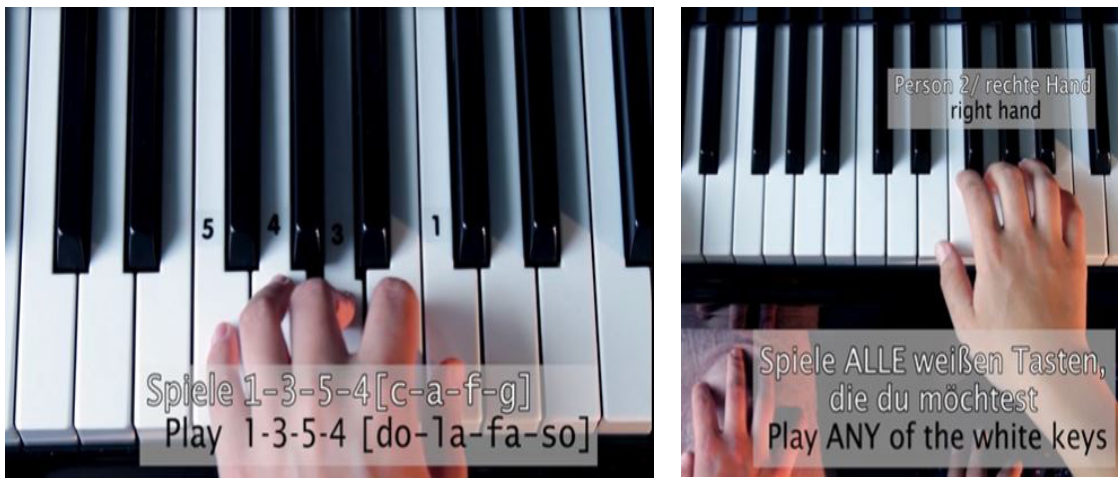

Abbildung 1/1 und Abbildung 1/2: Standbild aus dem Anleitungsfilm Play TWOgether (erstellt von Jourdan, E. (c) KlaWIR am Bahnhof GbR)

Zur besseren Veranschaulichung wird auf Deutsch und Englisch erklärt, was genau zu tun ist. Der Name Play TWOgether wurde gewählt, da dieser die Intention einer gemeinschaftsbildenden Aufforderung beinhaltet. Play TWOgether kann jedoch auch von einer einzelnen Person - in dem Fall mit der linken und der rechten Hand - gespielt werden. Mithilfe des QR-Codes und einem Smartphone hat man jederzeit Zugriff auf das Video. Zusätzlich gibt es Erklärungen, die am Klavier befestigt sind (Abb. 3), so dass es auch ohne Smartphone möglich ist, den Anweisungen zu folgen. Es handelt sich dabei um ein Spielen nach Zahlen - erste Schritte am Klavier.

Person 1/linke Hand:

Lege deine Finger auf die markierten Tasten

Finger: $\quad 1-3-5-4$ [c - a - f - g]

Spiele langsam und immer wiederholend

die Tasten 1-3-5-4...1-3-5-4 usw.

Wichtig: Bleibe in einem Tempo!

Tipp: Wenn es dir hilft, wippe mit dem Fuß mit.

Person 2/rechte Hand:

Spiele ALLE weißen Tasten, die du möchtest.

Deiner Fantasie sind keine Grenzen gesetzt!

Viel Spaß!

\section{Person 1/left hand:}

Put your fingers on the marked

keys, finger $\quad 1-3-5-4$ [do-la-fa-so]

Play slowly and always repeat the keys 1-3-5-4..1-3-5-4 and so on

Important: Keep to a single beat-if it helps, move your feet to the beat.

\section{Person 2/right hand:}

Play any of the white keys, feel free to be creative!

Have fun!

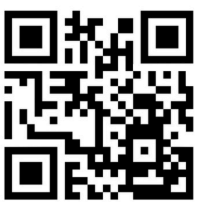

Abbildung 2: Konzept Play TWOgether (Mona Heiler, (c) KlaWIR am Bahnhof GbR) 
Das Projekt Play TWOgether, das ursprünglich Projekt KlavAnfängerInnen hieß, wurde von den Partnerinnen und Partnern und Sponsoren zunächst nicht bewilligt. Man wollte keine Anfängerinnen und Anfänger am Klavier, die evtl. Misstöne und einen weniger ästhetischen Klang produzieren. Jedoch ließe sich ohnehin nicht verhindern, dass auch Menschen ohne Vorkenntnisse und Anleitung das Instrument benützen würden. Die Spielanleitung folgt einem sehr einfachen Prinzip: Es werden alle beliebigen weißen Tasten zum Improvisieren und Kadenzen in C-Dur genutzt. Wird nach diesem Prinzip musiziert, so ergeben sich nur Klänge, die für das an europäische Hörgewohnheiten angepasste Ohr wohltönend sind. So entsteht ein Klangerlebnis, welches sowohl für die erstmals am Klavier Sitzenden als auch für ihre Umwelt bereichernd wirkt. Das nun bewilligte Projekt wurde von KlavAnfängerInnen in Play TWOgether umgetauft, wodurch die nach außen transportierte Botschaft des gemeinsamen Musizierens stärker gewichtet werden sollte, anstatt den Anschein $\mathrm{zu}$ vermitteln, dass es sich um ein didaktisches Konzept für Anfängerinnen und Anfänger handle.

\subsection{DienstagsKOMMbo und FreitagsOHRkester}

Zweimal in der Woche, von 17-19 Uhr, werden alle Menschen eingeladen, an den Bahnhof zu kommen, um in Form von Jam Sessions miteinander zu musizieren oder zuzuhören. Diese werden über Medien wie Zeitung und Radio, aber auch über Roll-Ups am Bahnhof sowie Flyer und Plakate in der ganzen Stadt angesprochen. Die Zielgruppe sind hier einzelne Personen, aber auch Musikgruppen, die Lust haben, andere Musikerinnen und Musiker zu treffen. Es sollen sich jedoch auch Menschen ohne musikalische Vorerfahrung angesprochen fühlen zu kommen. Wer mitmachen möchte, kommt mit oder ohne Instrument oder mit sonstigen klangerzeugenden Gegenständen (z. B. Kochlöffeln oder Töpfen). Mit den Jam Sessions wird das Ziel verfolgt, allen ein Mitmachen und Mitwirken gemäß der eigenen Möglichkeiten zu eröffnen und alle in den musikalischen Prozess einzubinden. Dies geschieht mithilfe einer*eines Musikvermittelnden des KlaWIR-am-Bahnhof-Teams, der*die diese Jam Sessions betreut und dabei die Teilnehmenden gegebenenfalls anleitet, motiviert und ermutigt. 


\subsection{Offene Proben}

Durch Pressearbeit, Marketing und direkte Anschreiben der KlaWIR-amBahnhof-Organisatorinnen an Chöre, Musikvereine und Bands sowie an Schulklassen wird die Möglichkeit gegeben, außerhalb der gewohnten Umgebung zu musizieren. Um am Bahnhof Musik zu machen, sind weder eine Voranmeldung noch konventionelle Bedingungen (wie zum Beispiel Dresscode/Konzertkleidung etc.) nötig. Das Ziel besteht nicht darin, einen Konzertcharakter aufkommen zu lassen, sondern das gemeinsame Musizieren, Ausprobieren und Proben in den Vordergrund zu stellen. Alle, die sich am Bahnhof aufhalten, sollen durch diese Offenen Proben mitbekommen, was in der Stadt und im Umkreis musikalisch passiert, jedoch oft verdeckt bleibt.

Die Proben am Bahnhof sind zudem dazu da, Raum für Musikmachen in legerer und entspannter Atmosphäre zu bieten. Die auf logistischen Gründen beruhende Tatsache, dass es keine Konzertstühle gibt, unterstützt die Idee, dass es den Leuten freigestellt ist, wann sie kommen und gehen wollen. Die Verbreitung einer positiven Stimmung am Bahnhof ist ein weiterer Aspekt, den die Offenen Proben beisteuern sollen. Reisende und sich am Bahnhof Aufhaltende können gemeinsam Musik machen und hören. Dadurch können sich gegebenenfalls neue Bekanntschaften ergeben.

\section{Evaluation des Projekts und Resonanzen}

Einige der Teilnehmenden bzw. Zuhörenden der MitMACHkonzerte kamen geplant zu den Veranstaltungen, viele von ihnen fanden sich spontan am Bahnhof zusammen. Manche blieben nur kurz stehen, andere verweilten das ganze Konzert über. Viele brachten sich dabei auf verschiedene Arten musikalisch ins Geschehen ein: Einige musizierten beispielsweise auf selbst mitgebrachten Instrumenten oder mit den dort zur Verfügung gestellten Percussion-Instrumenten. Andere wechselten sich mit dem Pianisten der Band am KlaWIR ab. ${ }^{3}$ Immer wieder wurde dabei den vorgegebenen Impulsen der Bandmitglieder gefolgt und so das Publikum interaktiv ins Konzertgeschehen eingebunden. ${ }^{4}$

Play TWOgether wurde von Passantinnen und Passanten im Rahmen des Projekts KlaWIR am Bahnhof sehr positiv angenommen. Dies zeigen sowohl Beobachtungsprotokolle, auf welchen die Nutzung des Klaviers beobachtet und dokumentiert wurde, als auch die Abrufzahlen des Videos, in dem die Musi-

3 Videobeitrag verfügbar unter: https:/www.facebook.com/KlaWIRamBahnhof/ videos/273608673255549/ [05.08.2020].

4 Videobeitrag verfügbar unter: https:/www.facebook.com/KlaWIRamBahnhof/ videos/729562277401413/ [05.08.2020]. 
zierenden entsprechende Anleitungen finden. Beides zeigt eindeutig, dass viele Teilnehmerinnen und Teilnehmer dem Musiziermodell sowohl über das Video als auch über die aufgeklebte Textanleitung folgten. ${ }^{5}$ Diese Anleitung wurde sowohl von Einzelpersonen umgesetzt als auch gemeinschaftlich von jeweils zwei Personen zusammen, wobei eine Person die linke und die andere Person die rechte Hand übernahm. Es wurde ebenfalls beobachtet, dass manche Passantinnen und Passanten, die diese Anleitung ausprobierten, scheinbar nicht zu einem für sie befriedigenden Ergebnis kamen. Dies lässt sich laut Beobachtungen darauf zurückführen, dass sie sich nicht genügend Zeit nahmen, um auf die einzelnen Schritte konzentriert einzugehen.

Während das Konzept PlayTWOgether ohne weitere Aktivität bzw. Intervention von außen umgesetzt werden konnte, stellte sich bald heraus, dass dies bei den Jam Sessions nicht funktionierte. Nicht, weil sich nicht genügend Mitmachfreudige zur genannten Uhrzeit einfanden. Vielmehr lag das Problem darin, dass die Personen Schwierigkeiten hatten, sich einander vorzustellen und die Jam Session ohne Anleitung zu eröffnen. Alle warteten, bis derdie Musikvermittler*in hinzukam und alle willkommen hieß. Daraufhin entstand eine neue Dynamik und nur nach kurzer Zeit vernetzten sich die Session-Teilnehmenden von selbst. Abhängig davon, welche Gestaltungs- und Musizierfreude die jeweilige Gruppe entwickelte, konnte sich der Musikvermittler oder die Musikvermittlerin dann entweder vollkommen zurückziehen, selbst mitspielen oder versuchen, die Jam Session so zu lenken, dass alle gemäß ihrer Möglichkeiten Teil des musikalischen Prozesses werden konnten. Unter diesem Blickwinkel lässt sich sagen, dass die DienstagsKOMMbos und FreitagsOHRkester ein besonders großes gemeinschaftsbildendes Potenzial in sich tragen. Dies zeigte sich zum Beispiel daran, dass die musizierenden Gruppen während der Jam Sessions teilweise von außen als feststehende Band gesehen wurden. Die regelmäßig stattfindenden Jam Sessions waren Anlass dafür, dass manche Teilnehmende wöchentlich zurückkehrten.

Verbale Äußerungen von Passantinnen und Passanten verdeutlichen, dass KlaWIR am Bahnhof im Laufe der Zeit fester Bestandteil ihres Alltags geworden ist. Es zeigte sich, dass Menschen unterschiedlicher sozialer und kultureller Hintergründe und aus verschiedenen Generationen am KlaWIR Gemeinschaften formten.

Die Offenen Proben wurden vor allem von Chören angenommen. Meist, so wurde von den Chören kommuniziert, wurden die Proben zur selben Uhrzeit und am selben Tag, an dem die Proben normalerweise stattfanden, abgehalten - nur eben im Bahnhof. Neben persönlichen Einladungen an Chöre in Form von E-Mails und Anrufen, die Proben an den Bahnhof zu legen, verbrei-

5 Videobeitrag verfügbar unter: https://vimeo.com/228489957 [05.08.2020]. 
tete sich diese neue Möglichkeit vor allem unter den Chören und Passantinnen und Passanten wie von selbst. Immer mehr Chormitglieder aus der Region erhielten diese Information durch die Präsenz des KlaWIRs im Stadtbild und durch Mund-zu-Mund-Propaganda. Die Chöre profitierten nicht nur von dem Angebot einer Offenen Probe, weil sich ihnen so die Chance bot, die Öffentlichkeit am Bahnhof zum Anwerben neuer Mitglieder zu nutzen, indem z. B. Flyer oder selbstgebackene Notenkekse verteilt wurden. Wie sich in Gesprächen mit Chorsängerinnen und Chorsängern herausstellte, empfanden sie vor allem den Akt des Singens im öffentlichen Raum als besonderes und außergewöhnliches Erlebnis. Denn obwohl explizit als Öffentliche Probe betitelt, wurde das Angebot von manchen Chören eher als Auftritt verstanden, mit dem Vorteil, dass mit diesem kein großer organisatorischer Aufwand verbunden war. Dies ließ sich daran erkennen, dass oft weniger an bestimmten Stücken geprobt wurde, sondern die Chöre stattdessen ihr ganzes Repertoire am Stück sangen oder viele Mitglieder in Auftrittskleidung erschienen.

Durch Fragebögen, die am Klavier auslagen, wurden folgende, exemplarische Meinungen erhoben: „Ich habe mit anderen Passanten geredet und zufällig Freunde getroffen.“ (Kita-Leiterin, 49 Jahre), „Eine sehr schöne Idee, die meinen Alltag verändert, Menschen zusammenbringt und mich wieder zum Musizieren bringt.“ (Lehrerin, 50), „Keiner, der Geld oder eine Gegenleistung möchte, sondern einfach die Idee, Menschen zusammenzubringen. Toll. Danke!“ (Student, 27 Jahre) und zum Beispiel: „Wunderbare Idee, die Menschen aus ihrer Anonymität zu holen!“ (Rentner, 76 Jahre). Eine Studentin (23 Jahre) erläuterte, dass es KlaWIR am Bahnhof möglich macht, bestimmte Menschen auf eine neue Art kennenzulernen, da diese, indem sie spielen, etwas Neues von sich Preis geben. Des Weiteren erzählte eine 24-jährige Studentin, die erst seit einer Weile Klavier spielt, dass sie mit „ihrem Liebsten und einem sehr feinen Reisenden“, den die beiden zufällig trafen, am KlaWIR spielte. Unter Das möchte ich zum Projekt ,KlaWIR am Bahnhof" noch sagen schrieb sie: „Musik verbindet, selbst um halb 2 nachts am Braunschweiger Bahnhof".

Diese Aussagen beziehen sich nicht immer im Direkten auf musikalische, sondern auch auf soziale Interaktionen. Die Passantinnen und Passanten, die Rückmeldung gaben, betonen überwiegend den sozialen Charakter des Projekts, der zwar durch musikalische Interaktion entstehen kann, aber nicht zwingenderweise muss. Die soziale Interaktion kann zum Beispiel genauso über gemeinsames Zuhören oder verbale Kommunikation entstehen. Die Feedbacks der Passantinnen und Passanten, die diese soziale Komponente thematisierten, stehen dabei oft in Verbindung mit dem Ausdruck positiver Emotionen, immer wieder mit dem Wunsch, ein Klavier am Bahnhof als dauerhaftes Ereignis stehen zu haben oder mit Vorschlägen, diese Idee auch an anderen Bahnhöfen und in sozialen Einrichtungen umzusetzen. 
Auch der Standort des Projekts wurde in den Evaluationen thematisiert: So sprachen Passantinnen und Passanten in ihren Rückmeldungen über die Verwandlung des Bahnhofs durch das Projekt oder nannten dabei die Außergewöhnlichkeit des Ortes Bahnhof: „DANKE, es ist ein schöner Zeitvertreib in dieser Nacht, wo alle Züge stillstehen, Klavier zu spielen! Vor allem in diesem surrealen Setting ... wunderbar!“ (Passantin, anonym). Das Projekt KlaWIR am Bahnhof führt zu einer Umdeutung des Raums, weg von einem Ort des reinen Passierens oder Wartens, hin zu einem Ort des aktiven Erlebens.

Auch die musikalische Gewichtung der Interaktion wird in einigen Reaktionen der Feedback-Bögen und Nachrichten wiedergefunden. So zum Beispiel die Aussage, dass das KlaWIR die Musizierlust erwecke (Lehrerin, 43).

Des Weiteren wurde ein persönlicher Brief in die Feedback-Box eingeworfen. Auf diesem steht: „toll, macht Spaß, ich hab gesungen und gespi[e]lt, eine [B] and hat gespi[e]lt mit mir"6. Auch Play TWOgether, als ein Konzept der musikalischen Interaktion, wurde positiv kommentiert: „Eine super schöne Idee!!! Besonders mit der Anleitung für Anfänger!“ und KlaWIR hört sich gut an, Anleitung ist toll!“ (Schüler, 11 Jahre). Zudem soll erwähnt sein, dass Feedback-Briefe in arabischer, türkischer, englischer und spanischer Sprache eingeworfen wurden. Es konnte festgestellt werden, dass das Projekt von Menschen jeglichen Alters und jeglicher Herkunft angenommen wurde. Dies zeigen zum einen die Altersangaben, zum anderen bestätigt sich dies durch die soeben genannten Feedbackbriefe, die nicht nur in deutscher Sprache verfasst worden sind.

Während KlaWIR am Bahnhof ergaben sich gemeinschaftsbildende Prozesse unterschiedlichster Dauer und Intensität. ${ }^{7}$ Bei manchen handelte es sich um kurze Momentaufnahmen; Begegnungen, die lediglich durch nonverbale Interaktionen stattfanden, bei anderen jedoch um ein regelmäßiges Wiedersehen am KlaWIR und stundenlange gemeinsame musikalische und soziale Interaktion.

Am KlaWIR bildeten sich verschiedene Formen der Gemeinschaft: so zum Beispiel im Rahmen der DienstagsKOMMbo oder des FreitagsOHRkesters, bei denen die Bildung von Gemeinschaften in Form von aktiver Teilnahme vorgesehen war oder beim freien Musizieren, bei dem sich Gemeinschaften spontan entwickelten. Die Auswertungen der Fragebögen ergaben, dass die musi-

6 Wie die Rechtschreibfehler und die in Schreibschrift geschriebene Nachricht vermuten lässt, handelt es sich um das Feedback eines Schulkindes, das am KlaWIR musizierte.

7 Es wurde lediglich auf temporäre Prozesse der Gemeinschaftsbildung Bezug genommen, da nicht wissenschaftlich gemessen werden konnte, inwiefern auch nach dem beobachteten Zeitraum sich am KlaWIR entwickelte Gemeinschaften bestehen blieben. 
kalische Interaktion, sei es bei Play TWOgether oder beim freien Musizieren, überwiegend zwischen Menschen stattfand, die sich zuvor noch nicht kannten. Gespräche, sowohl von kurzer als auch von langer Dauer, waren am KlaWIR nicht selten. Die Antworten der Fragebögen und Feedback-Briefe der Passantinnen und Passanten zeigen deutlich, dass das KlaWIR als Phänomen wahrgenommen wurde, welches Begegnungen zwischen Menschen ermöglicht hat. Generell kann festgestellt werden, dass das KlaWIR zu gemeinschaftsstiftenden musikalischen und sozialen Erlebnissen führt: sei es von Menschengruppen, die zusammen ans KlaWIR kamen, von einzelnen Menschen, die sich kannten und zufällig am KlaWIR aufeinander trafen, oder auch von Personen, die sich zum ersten Mal in ihrem Leben am KlaWIR begegneten.

Abschließend kann eindeutig festgestellt werden, dass KlaWIR am Bahnhof über großes gemeinschaftsbildendes Potenzial auf diversen Ebenen verfügt.

\section{Fazit und Ausblick}

Ausgehend von Erfahrungen in Frankreich, wo an vielen Bahnhöfen im ganzen Land Klaviere zum freien Spielen bereit stehen, wurde die Initiative KlaWIR am Bahnhof in Deutschland ins Leben gerufen. Anhand von verschiedenen neu konzipierten Musikvermittlungsprojekten und Aktionen konnte das Projekt an deutschen Bahnhöfen stetig weiterentwickelt werden. $\mathrm{Zu}$ diesen zählen niedrigschwellige Projekte wie die MitMACHkonzerte und die Installation Play TWOgether, die jeweils Menschen ohne musikalische Vorerfahrungen die Möglichkeit geben, gemeinsam zu musizieren, die DienstagsKOMMbos und FreitagsOHRkester, bei denen alle mitmachen können, die Lust haben, oder die Offenen Proben, die Musikgruppen, Vereinen, Schulklassen und Chören aus der Region die Möglichkeit geben, am Bahnhof, einem Ort der Öffentlichkeit, zu proben, sich zu präsentieren, sich auszutauschen und für ihre Institution zu werben. Die Forschung vom Projekt KlaWIR am Bahnhof zeigt, dass allein durch das KlaWIR, auch ohne die Anwesenheit von Musikvermittlerinnen und Musikvermittlern gemeinschaftsbildende Prozesse ausgelöst werden können. Die einzelnen Vermittlungsprojekte von KlaWIR am Bahnhof verdeutlichen jedoch, wie die pädagogische Begleitung der Musikvermittlerinnen und Musikvermittler das gemeinsame Musizieren fördern und gleichzeitig das gemeinschaftsbildende Potenzial des Projektes um Dimensionen erweitern. Ein weiterer wichtiger Punkt ist, dass die Örtlichkeit Bahnhof permanent eine zentrale Rolle im Geschehen einnimmt. Der Bahnhof als sozialer Raum wird zum Ort verschiedenster nonverbaler, verbaler und musikalischer Begegnungen. Hierbei lassen sich aufeinander aufbauende Phasen der Kommunikation und Interaktion erkennen: Nach nonverbalen zeigten sich oft verbale Reaktio- 
nen und soziale Interaktionen, welche in musikalische mündeten. Durch das gemeinsame Zuhören, durch Gespräche und/oder das zusammen Musizieren entwickelten sich so letztlich Gemeinschaften. Die Musikvermittlungsprojekte von KlaWIR am Bahnhof sind auf verschiedenen Ebenen gemeinschaftsfördernd und lassen alle Menschen - egal mit welchem Hintergrund und welcher musikalischer Vorerfahrung - teilhaben. Dies zeugt vom inklusiven Charakter des Projekts.

Nach drei erfolgreichen Projektjahren von KlaWIR am Bahnhof ist mit den Kooperationspartnerinnen und -partnern eine Fortsetzung und Ausweitung des Projekts im Gespräch. Aufgrund des hohen finanziellen und logistischen Aufwands wird es bei KlaWIR am Bahnhof jedoch vorerst bei Projekten bleiben, dessen Durchführung an den jeweiligen Bahnhöfen auf wenige Wochen oder Monate begrenzt ist. Die Möglichkeit, KlaWIR am Bahnhof als ein dauerhaftes Projekt durchzuführen, würde das andauernde gemeinsame Musizieren vieler Menschen erheblich fördern und sich noch nachhaltiger auf die Stadtgesellschaft auswirken können. Vor allem die Entwicklung der DienstagsKOMMbos und FreitagsOHRkester mit ihren feststehenden Terminen braucht Zeit, denn diese müssen sich im Alltag beziehungsweise in der Wochenroutine der Passantinnen und Passanten und Bürgerinnen und Bürger der Stadt festigen. Diese Regelmäßigkeit würde ggf. mit sich bringen, dass die Hemmschwelle sinken und man sich neben den einzelnen Musikvermittlungsprojekten mit Klavierspielen beziehungsweise generell mit Musikmachen mehr beschäftigen würde. Außerdem würde diese, gemeinsam mit dem innovativen Potenzial des Projekts, eine konstante und längerfristige Auseinandersetzung mit dem neuen Musizierangebot ermöglichen. Der Bahnhof ist ein kulturelles Terrain, das als ein Ort der Anonymität durch KlaWIR am Bahnhof die Chance hat, sich mehr und mehr in einen Raum des Austauschs und der Gemeinschaftsbildung zu verwandeln.

\section{Literatur}

de Banffy-Hall, A. (2018). Developing Community Music in Munich. Dissertation, Hochschule für Musik und Theater München. 



\section{POWERband Tirol: eine inklusive Band im Fokus}

\section{Abstract}

In dem vorliegenden Artikel wird die partizipative Evaluation der POWERband Tirol mit dem Fokus auf die Partizipationsmöglichkeiten der Musiker*innen mit geistiger Behinderung beschrieben. Die Band wurde $2011 \mathrm{mit}$ der Idee eines inklusiven Freizeitprojekts gegründet. Aus der öffentlichkeitswirksamen Darstellung konnten in Hinblick auf ein der UN-BRK angelehntes Inklusionsverständnis Unschärfen identifiziert werden, die den ausschlaggebenden Anlass für die vorliegende Studie bildeten. Als Forschungsmethode wurde eine partizipative Herangehensweise gewählt, die in enger Kooperation mit dem Vereinsvorstand entlang eines Partizipationsstufenmodells durchgeführt wurde. Im Zuge der Erarbeitung bildeten sich zwei Betrachtungsbereiche heraus, die getrennt voneinander bearbeitet und ausgewertet wurden. Die Ergebnisse zeigten einerseits Defizite im strukturellen und organisatorischen Bereich, andererseits eine sehr fortgeschrittene Partizipation und somit gelingende Inklusion im Bereich des musikalischen Momentes beim Musizieren. Aus diesen Erkenntnissen wurden Maßnahmen definiert, die zum Teil bereits umgesetzt wurden.

\section{Einleitung}

Aktives Musizieren ist eine spezielle Form der Freizeitbeschäftigung, die nach eigener Erfahrung eine äußerst wertvolle Bereicherung im Leben und der persönlichen Entwicklung darstellt. Menschen mit Behinderung haben nicht immer zu gleichen Teilen die Möglichkeit, an solchen Freizeitaktivitäten, wie beispielsweise Bandprojekten, teilzuhaben. Dies kann einerseits daher rühren, dass diesen Menschen die Unterstellung mangelnder Kompetenz bzw. Begabung anhaftet oder einfach nur Angebote den Bereich der Behinderung unberücksichtigt lassen (vgl. dazu Krebber-Steinberger, 2014).

Im Rahmen des Projekts POWERband Tirol wurde das Ziel verfolgt, für Menschen mit Behinderung ein solches Freizeitangebot zu schaffen, Denkweisen, die darauf zielen, aktives Musizieren einer begabten Elite zuzuschreiben, auszuklammern und an die musikalischen Ausdrucksmöglichkeiten eines jeden Menschen zu glauben. 
In dem vorliegenden Artikel wird eine Evaluationsstudie vorgestellt, mittels derer Partizipationsprozesse innerhalb des Bandprojektes untersucht wurden und der Inklusionsgrad vor dem Hintergrund des Behinderungsverständnisses des Übereinkommens über die Rechte von Menschen mit Behinderung $(\mathrm{UN}-\mathrm{BRK})^{1}$ geprüft wurde. Dabei wurden als Hilfsmittel die Partizipationsstufen nach Wright, Block, Kilian und Lemmen (2013) verwendet und als Forschungsmethode ein partizipativer Ansatz im Sinne der Aktionsforschung verfolgt.

Das Ziel der Studie war es, ein detailliertes Bild davon zu zeichnen, inwiefern Partizipation einerseits im strukturellen Organisationsbereich, andererseits im Moment der musikalischen Darbietung gewährt wird. ${ }^{2}$

\section{Die POWERband Tirol}

Die POWERband Tirol wurde im Jahre 2011 vom Tiroler Jazzmusiker und Sozialpädagogen Christoph Heiß mit der Idee gegründet, dass Menschen mit und ohne Behinderung gemeinsam musizieren sollten. In den Anfangszeiten der POWERband Tirol wurde noch in Räumlichkeiten der Lebenshilfe Tirol geprobt. Der erste Auftritt wurde in Innsbruck im Treibhaus bestritten. Hier trat die Gruppe als Begleitband im Kontext einer musikalischen Abschlussprüfung des Landeskonservatoriums Tirol auf. Im Jahre 2012 wurde versucht, die POWERband Tirol als ein inklusives Musikprojekt innerhalb der Lebenshilfe Tirol zu installieren. Da sich das nicht realisieren ließ, wurde im Dezember 2012 der eigenständige und institutionell unabhängige Verein der POWERband Tirol gegründet. Der Vorstand bestand bei der Gründung aus Eltern von Musiker( ${ }^{*}$ inne)n mit Behinderung und Menschen, die das Projekt aus ihrer persönlichen Überzeugung und Haltung heraus unterstützen und begleiten wollten. Im Zuge dieser Gründung wechselte die Band den Proberaum und erhielt im umliegenden Flüchtlingsheim einen Raum, der von Sponsoren gefördert und von Asylwerber $\left({ }^{*}\right.$ inne)n umgebaut wurde. Seitdem wird im neuen Proberaum regelmäßig geprobt. Zudem werden auch stetig Konzerte gespielt,

1 Das Übereinkommen wurde 2006 von den Vereinten Nationen verabschiedet und von Österreich 2008 ratifiziert. Siehe dazu: https:/www.sozialministerium.at/ Themen/Soziales/Menschen-mit-Behinderungen/UN-Behindertenrechtskonvention. html [05.08.2020].

2 Sämtliche Ergebnisse der Studie wurden im Zuge einer Qualifikationsarbeit an der Universität Innsbruck publiziert. 
wie beispielsweise das Konzert im Rahmen des Tschirgart Jazzfestivals Imst 2012, bei dem die POWERband Tirol im Vorprogramm von Al Jarreau ${ }^{3}$ spielte.

Die Band besteht aktuell aus acht Musiker( ${ }^{*}$ inne)n (zwei Frauen und sechs Männern) sowie dem Ensembleleiter Christoph Heiß. Für Konzerte werden professionelle Musiker*innen engagiert, welche die Band musikalisch unterstützen. Die acht regulären Musikerinnen sind Menschen mit Behinderung. Bei allen liegt eine geistige Beeinträchtigung vor, bei zwei Musikern zusätzliche körperliche Einschränkungen (Funktionsstörungen der Hände bzw. der Beine).

Die Instrumentierung der Band im Livebetrieb setzt sich wie folgt zusammen:

\begin{tabular}{|c|c|}
\hline $\begin{array}{l}\text { Instrumentierung (reguläre } \\
\text { Musiker*innen) }\end{array}$ & $\begin{array}{l}\text { Instrumentierung angemieteter } \\
\text { Profimusiker*innen (variiert nach Bedarf) }\end{array}$ \\
\hline 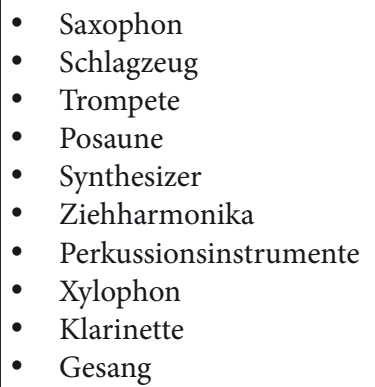 & $\begin{array}{ll}\text { - } & \text { Bassgitarre } \\
\text { - } & \text { Elektropiano } \\
\text { - } & \text { Schlagzeug } \\
\text { - } & \text { Gitarre }\end{array}$ \\
\hline
\end{tabular}

Tabelle 1: Instrumentierung der POWERband Tirol

Die POWERband Tirol probt regelmäßig in einem 14-tägigen Abstand. Als musikalische Unterstützung für die Proben verwendet die Band sogenannte Playbacks. Diese werden in einem Tonstudio aufgenommen und beinhalten die wichtigsten Tonspuren wie Schlagzeug, Bassgitarre, Elektropiano sowie Gesang. Dadurch ist die Band bei den Proben flexibel und kann auch abwesende Musiker*innen (vor allem die hinzukommenden Profimusiker*innen) kompensieren. Bei Live-Konzerten werden diese Tonspuren dann durch die Profimusiker*innen ersetzt.

3 Al Jarreau war ein US-amerikanischer Musiker, der vor allem Ende des 20 Jhd. internationale Bekanntheit erlangte (Siehe dazu auch: https://de.wikipedia.org/wiki/ Al_Jarreau [05.08.2020]). 


\section{Inklusion im Kontext der POWERband Tirol}

Für die vorliegende Studie wurde ein qualitatives Forschungsdesign mit Fokus auf größtmöglicher Partizipation der beteiligten Personen gewählt. Die Datenerhebung wurde größtenteils mittels der Methode der partizipativen Forschung durchgeführt, zu einem weiteren Teil wurde eine leitfadengestützte Gruppendiskussion mit den Musiker(*inne)n durchgeführt. Die Datenauswertung erfolgte durch die Methode der qualitativen Inhaltsanalyse nach Kuckartz (2014). Als Instrument für die Datenerhebung des partizipativen Forschungsteils wurde das Stufenmodell nach Wright et al. (2013) adaptiert, das für die Evaluation der Partizipationsmöglichkeiten von Menschen mit Behinderung innerhalb der POWERband Tirol ein geeignetes Instrument darstellte.

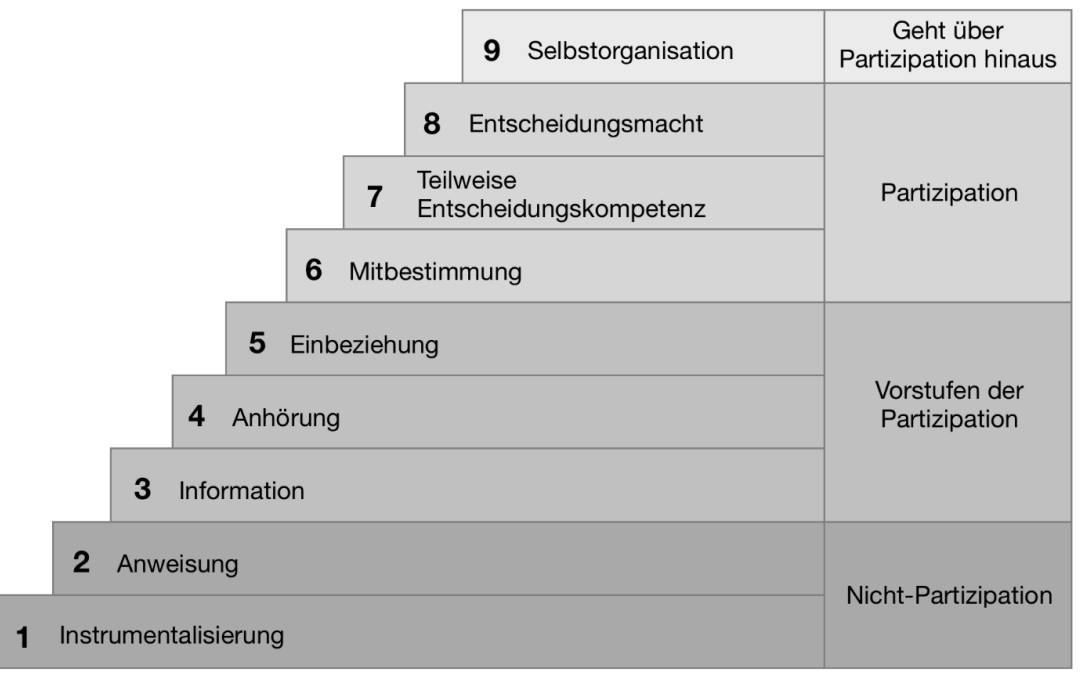

Abbildung 1: Partizipationsstufen (vgl. Wright et al., 2013, S. 152)

\subsection{Partizipative Forschung}

In dieser, der Aktionsforschung entsprungenen, partizipativen Forschungsmethode geht es in erster Linie darum, soziale Wirklichkeiten zu verstehen, zu beforschen und zu beeinflussen. Dies geschieht, indem der „[...] Anspruch der Partizipation auf Forschung [...] übertragen [wird]. Dadurch verändern sich Machtverhältnisse zwischen ForscherInnen und Beforschten“ (Flieger, 2017, S. 180). Diese Handlungsfelder werden in einer partnerschaftlichen Kooperation mit sogenannten Co-Forscher $\left({ }^{*}\right.$ inne)n durchgeführt, wodurch gesell- 
schaftliche Teilhabeprozesse gefördert werden sollen (vgl. von Unger, 2014, S. 1). Im besten Falle stellt der Ansatz der partizipativen Forschung für alle Seiten einen deutlichen Gewinn dar. Einerseits der Erkenntnisgewinn aus der sozialen Lebens- und Arbeitspraxis, andererseits die Möglichkeit für die CoForschenden, aus gewohnten Routinen auszusteigen und mit einem distanzierten, theoriegeleiteten Blick auf das Arbeitsfeld reflexive Prozesse in Gang zu setzen (vgl. Bergold \& Thomas, 2012).

\subsection{Forschungsziel}

Das Ziel der Untersuchung war es, durch die partizipative Erhebung der Teilhabe der Musiker*innen an Aktivitäten der POWERband Tirol den Grad der Partizipation zu ermitteln, diesen in Bezug zu dem der UN-BRK entsprungenen Inklusionsverständnis zu stellen und Beispiele für mögliche Optimierungsmaßnahmen herauszuarbeiten. Dabei wurden zwei große Bereiche beleuchtet, die sich im Zuge der partizipativen Datenerhebung verdichtet haben: einerseits die Partizipation an der Vereinsstruktur und den organisatorischen Prozessen innerhalb der Band und des Vereinsgeschehens, andererseits die Partizipation innerhalb des Moments der musikalischen Darbietung. Daraus entwickelte sich folgende zentrale Fragestellung: Welche Möglichkeiten der Partizipation bestehen für die Musiker*innen der POWERband Tirol in struktureller bzw. musikalischer Hinsicht?

\subsection{Forschungsprozess}

Um den partizipativen Forschungsprozess in Gang zu setzen wurde in einem ersten Schritt eine theoretisch-methodische Abgrenzung vorgenommen. Für die Beteiligung von Partner( ${ }^{*}$ inne)n, in Bezug auf die Forschungsziele und in Abhängigkeit zu den zeitlichen wie inhaltlichen Ressourcen, die zur Verfügung standen, schien folgende Positionierung als hilfreich: Das Participatory Action Research Modell (vgl. von Unger, 2014; Bergold \& Thomas, 2012) bot sich insofern an, da der Fokus hinsichtlich der Beteiligung am Forschungsprozess auf den Entscheidungsträger $\left({ }^{*}\right.$ inne)n lag und weniger auf den Musiker $\left({ }^{*}\right.$ inne)n. Des Weiteren wurde der Fokus auf die Aktionsforschung unter dem Dach der Praxisforschung (vgl. von Unger, 2014, S. 24) gelegt, da es um eine enge kooperative Zusammenarbeit ging und ein wesentliches Ziel der Forschung die Verbesserung und Weiterentwicklung der Praxis war. Dieses Ziel wurde partizipativ definiert und konsequent verfolgt. Als Methode wurde die Gruppendis- 
kussion gewählt, auch vor dem Hintergrund, gruppendynamische Prozesse mit in den Erhebungsprozess zu integrieren (vgl. Flick, 2014, S. 250). Mitglieder einer Institution, die zu Partner( ${ }^{\star}$ inne)n eines Forschungsprozesses werden, haben im Regelfall wenig Erfahrung mit Forschungsprozessen. Aus diesem Grund sind Schulungen zu Forschungsmethoden notwendig. ${ }^{4}$ Im vorliegenden Fall diente der Vorstand des Vereins als die Gruppe der Co-Forschenden. Dieser bestand zu diesem Zeitpunkt aus fünf Personen (drei Frauen und zwei Männern), vier (zwei Frauen und zwei Männer) davon nahmen am Forschungsprozess als CO-Forscher ${ }^{\star}$ innen teil.

\subsubsection{Datenerhebung mittels partizipativer Gruppendiskussion}

Die Datenerhebung gliederte sich in zwei große Teile, einerseits die partizipative Erhebung mit den Vorstandsmitgliedern des Vereins bzw. ein zweiter Teil mit dem Fokus auf den musikalischen Moment mit zwei ausgewählten Personen des Vereinsvorstands, andererseits eine leitfadengestützte Gruppendiskussion mit den Musiker( ${ }^{*}$ inne)n der POWERband Tirol.

Für den ersten Teil der Erhebung wurde mit dem Instrument der Partizipationsstufen nach Wright et al. (2013) gearbeitet, welches im Zuge der oben erwähnten Einschulung im Detail erläutert wurde.

Der erste Schritt zur Arbeit mit den Partizipationsstufen war die gemeinsame Definition der Zielgruppe bzw. der Gruppe der Entscheidungstragenden. Die Gruppe war sich einig, dass vor allem Mitspracherecht und Entscheidungsmacht Bereiche sind, die speziell für die Musiker*innen mit Behinderung diskutiert werden mussten. Als Zielgruppe wurden demnach die Musiker*innen der POWERband Tirol definiert, die bezahlten Profimusiker*innen wurden aus dieser Betrachtung herausgenommen.

Im nächsten Schritt wurden in gemeinsamer Arbeit die Kategorien bzw. Stufen der Partizipation, in Anlehnung an die Stufenbeschreibung nach Wright, Block und von Unger (2008), für die Zielgruppe definiert. Für die Erarbeitung dieser Kategorien wurde vom Autor eine Hilfstabelle vorbereitet. In dieser Tabelle wurden die Stufen der Partizipation in die fünf partizipativ erarbeiteten Kategorien Entscheidungskompetenz, Interesse, Mitspracherecht, Information und Verantwortung (deduktive Kategorien) unterteilt und gemäß

4 Die einführende Schulung der Co-Forscher*innen wurde in einer ersten Sitzung mit dem Vereinsvorstand mittels Vortrag und Präsentation zu den methodischen Inhalten durchgeführt. Ebenso wurde über die Rolle der einzelnen involvierten Personen gesprochen. Abschließend erfolgte in einem partizipativen Prozess die Definition des Forschungszieles und die Planung der weiteren Vorgehensweise. 
den Stufen definiert. Dabei wurde für jede Stufe und jede Kategorie eine Codierung erarbeitet.

\begin{tabular}{|c|l|l|}
\hline \multicolumn{1}{|c|}{ Kategorie } & $\begin{array}{l}\text { Abstufung im Sinne der Partizipationsstufen nach Wright et al. } \\
(2013)\end{array}$ & \multicolumn{1}{|c|}{ Code } \\
\hline $\begin{array}{l}\text { Entscheidungs- } \\
\text { kompetenz (A) }\end{array}$ & Alleinige Entscheidungskompetenz & A_a1 \\
\hline \multirow{5}{*}{} & $\begin{array}{l}\text { Zielgruppe bestimmt wesentliche Aspekte einer Maßnahme } \\
\text { selbst. }\end{array}$ & A_b1 \\
\cline { 2 - 3 } & $\begin{array}{l}\text { Teilweise Entscheidungskompetenz durch Vertreter der Ziel- } \\
\text { gruppe }\end{array}$ & A_b2 \\
\cline { 2 - 3 } & $\begin{array}{l}\text { Entscheidungen werden beeinflusst } \\
\text { Vertreter der Zielgruppe sind als BeraterInnen tätig, haben } \\
\text { jedoch keinen verbindlichen Einfluss. }\end{array}$ & A_c1 \\
\cline { 2 - 3 } & $\begin{array}{l}\text { Keine Gewährleistung, dass Sichtweisen Berücksichtigung } \\
\text { finden (keine aktive Entscheidungsmacht) }\end{array}$ & A_c2 \\
\cline { 2 - 3 } & $\begin{array}{l}\text { Die Zielgruppe hat nur geringen Entscheidungsspielraum } \\
\text { (Empfehlungen) }\end{array}$ & A_c3 \\
\cline { 2 - 3 } & $\begin{array}{l}\text { Die Zielgruppe hat keine Entscheidungskompetenz } \\
\text { Die Zielgruppe hat keine Entscheidungskompetenz }\end{array}$ & A_d1 \\
\hline
\end{tabular}

Tabelle 2: Ausschnitt Hilfstabelle Partizipationskategorien in Anlehnung an Wright et al. (2008) (vollständige Tabelle: Greuter, 2017)

Im Verlauf der ersten Diskussionsrunde wurde vom Großteil der Vereinsmitglieder die Zuordnung der einzelnen Stufen im Wesentlichen auf strukturelle und organisatorische Bereiche reduziert. Aus dieser Fokussierung heraus entstand ein Spannungsverhältnis zwischen den strukturellen Prozessen und der Betrachtung musikalischer Prozesse. Der entscheidende Impuls für die dezidierte Unterscheidung der Betrachtung kam vom Ensembleleiter, der den Bereich der Musik als das Kernprojekt der Inklusion innerhalb der POWERband Tirol definierte. Dies führte dazu, dass am Ende der ersten Sitzung beschlossen wurde, dem Moment der musikalischen Darbietung eine eigene Betrachtung $\mathrm{zu}$ widmen und mit denselben inhaltlichen Prozessen zu arbeiten.

\subsubsection{Gruppendiskussion mit Menschen mit geistiger Behinderung}

In einem weiteren Schritt wurde eine Gruppendiskussion mit den Musiker ${ }^{*}$ inne)n mit Beeinträchtigung bezugnehmend auf die Ergebnisse der Arbeit mit den Entscheidungsträger ${ }^{*}$ inne$) n$ vorbereitet und durchgeführt. 
Qualitative Interviewformen im Kontext von Menschen mit geistiger Behinderung stellen ein eigenes methodisches Feld dar, das beispielsweise von Jutta Hagen (2002) ausführlich beschrieben wurde. Dabei erläutert sie, wie ein ideales Setting bei Interviews mit Menschen mit geistiger Behinderung aussehen kann. Grundsätzlich ist es wichtig darauf zu achten, dass bei den Beteiligten nicht der Eindruck entsteht, einer Testsituation ausgesetzt zu sein, da Menschen mit geistiger Behinderung in ihrem Leben häufig mit solchen Situationen konfrontiert werden und dies meist negativ konnotiert ist. Für eine gelingende Arbeit ist es laut Hagen (2002, S. 299) empfehlenswert, im Vorfeld ein Vertrauensverhältnis aufzubauen, um die Interviewsituation etwas zu entspannen. Aber auch die Auseinandersetzung mit der geeigneten Fragetechnik ist unumgänglich, da sich Interviewsituationen mit Menschen mit geistiger Behinderung grundsätzlich anders entwickeln können als mit Menschen ohne geistiger Behinderung. Dazu schreibt Niediek: Damit „die befragte Person durch die Zusammenarbeit mit dem Interviewer in einer vertrauensvollen Gesprächsatmosphäre ihre eigene Problemsicht entfalten“ (Niediek, 2014, S. 101) kann, ist es notwendig, durch gezielte Techniken auf die Situation Einfluss zu nehmen, beispielsweise dienen Bilder sehr gut als Gesprächseinstieg. Es empfiehlt sich ferner, den Fokus auf stärker öffnende Formulierungen anstatt auf eingrenzende Fragen zu setzen. Hingegen sollten bestätigende Signale eher sparsam eingesetzt werden, da ansonsten schnell der Eindruck entsteht, dass der Sachverhalt bereits ausreichend geschildert wurde. Stattdessen sollte mit Mimik und Gestik signalisiert werden, dass noch weitere Informationen wünschenswert sind. Vor allem bei Personen, die wenig erzählen, dient eine abwartende Haltung als gesprächsfördernd. Dagegen sollte eine vorzeitige Wiederholung der Frage vermieden werden (vgl. Niediek, 2014, S. 100-104).

Das Vertrauen der Musiker*innen zu mir als Interviewführenden war im Fall der POWERband Tirol insofern vorhanden, da ich bereits ein Jahr zuvor als Gastmusiker tätig war und somit die Musiker*innen gut kannte und bereits Einblicke in die gruppeninternen Strukturen gewonnen hatte.

Bei der Gruppendiskussion waren sieben Musikerinnen sowie der Ensembleleiter und der Obmann des Vereines anwesend. Die Diskussion fand in vertrauter Umgebung im Proberaum der Band statt. Die Diskussion selbst gestaltete sich äußerst spannend, da über eine Stunde lang eine hochkonzentrierte Atmosphäre herrschte und sich alle Musiker ${ }^{*}$ innen am Gespräch beteiligten. Manche Musiker*innen nahmen dabei mehr Raum ein, bei ein paar wenigen war es notwendig, sie durch gezieltes Ansprechen zur Beteiligung am Gespräch zu ermutigen. Für die Einleitung der Sitzung wurde ein Begrüßungstext 
in leichter Sprache ${ }^{5}$ vorbereitet, die Fragen wurden in Form eines Leitfadens ebenfalls in leichter Sprache formuliert (vgl. Greuter, 2017).

\section{Ergebnisse}

Der erste Teil der Auswertung beschäftigte sich mit der partizipativen Erhebung der Partizipationsstufen nach Wright et al. (2013) entlang des deduktiv formulierten Kategorienschemas (Entscheidungskompetenz, Interesse, Mitspracherecht, Information und Verantwortung). Anschließend wurden weitere induktiv gebildete Kategorien anhand des Datenmaterials ausgewertet und interpretiert.

Die Einstufung der Partizipation mittels des Instrumentes der Partizipationsstufen nach Wright et al. (2013) hat einen sehr detaillierten Einblick in die Strukturen der POWERband Tirol ermöglicht. Für den Bereich der Organisation bzw. der Verwaltung wurde ein sehr geringer Grad der Partizipation festgestellt, für den Bereich der musikalischen Darbietung war das Gegenteil der Fall, hier zeigten die Ergebnisse deutlich, dass eine sehr hohe Stufe der Partizipation aller Musiker*innen erreicht wird. Die von Krebber-Steinberger (2014) beschriebenen Barrieren im Kopf scheinen in musikalischer Hinsicht weitgehend überwunden. Im Bereich der Organisationsstruktur kann hingegen noch ein defizitorientiertes Verständnis festgemacht werden. Hier wäre ein gedanklicher Paradigmenwechsel, im Sinne der UN-BRK von einem Fürsorgedenken zu einer Haltung der Rechte, notwendig (vgl. Aichele, 2010). Insbesondere, um dem Anspruch der Inklusion auf allen Ebenen gerecht zu werden. Denn die Gefahr, Inklusion als inflationären In-Begriff zu verwenden ist durchaus gegeben (vgl. Hinz, 2008, S. 37). Eine Schärfung des Inklusionsverständnisses, beispielsweise mittels weiterbildender Maßnahmen, könnte hier hilfreich sein.

Interessant an der Auseinandersetzung mit den partizipativ erarbeiteten Kategorien für den musikalischen Bereich entlang der Partizipationsstufen nach Wright et al. (2013) ist die Erkenntnis, dass ein höherer musikalischer Professionalisierungsgrad zwar partizipative Prozesse grundsätzlich verstärkt ermöglicht, der Unterschied der Teilhabe im Sinne der musikalischen Partizipation allerdings verhältnismäßig gering ausfällt. Folgende Gegenüberstellung

5 Leichte Sprache ist ein spezielles Konzept für Menschen mit Lernschwierigkeiten, um schwerverständliche Texte zugänglich zu gestalten. Dieses Konzept folgt einem festen Regelwerk. Texte in leichter Sprache werden von Menschen mit Lernschwierigkeit auf Verständlichkeit geprüft (siehe dazu auch: https://www.leichtesprache.org/ [05.08.2020]). 
in der Einstufung der Kategorien anhand des Partizipationsstufenmodells soll dies verdeutlichen:

\begin{tabular}{|l|l|l|}
\hline Kategorie & Musikerinnen mit geistiger Behinderung & Profimusiker*innen \\
\hline Entscheidungsmacht & Stufe 7 & Stufe 8 \\
\hline Interesse & Stufe 6 & Stufe 9 \\
\hline Mitspracherecht & Stufe 8 & Stufe 8 \\
\hline Information & Stufe 7 & Stufe 8 \\
\hline Verantwortung & Stufe 4 & Stufe 8 \\
\hline
\end{tabular}

Tabelle 3: Einstufung entlang der Partizipationsstufen nach Wright et al. (2013) der Musiker*innen mit bzw. ohne Behinderung

Diese vergleichende Gegenüberstellung wurde im zweiten Termin erarbeitet und diente primär als Kontrollinstrument, um die Partizipation innerhalb der Gruppen zu vergleichen. Die Unterscheidung im Bereich der Interessen kam dadurch zustande, da als primäres Interesse der Profimusiker ${ }^{\star} i n n e n$ die Unterstützung eines sozialen Projektes und als sekundäres die Bezahlung definiert wurde. Beide Interessen werden wahrgenommen und zu vollen Teilen erfüllt, wobei das Interesse der Unterstützung wieder auf eine Fürsorgehaltung schließen lässt und das oben beschriebene Spannungsfeld zum Inklusionsbegriff verstärkt. Bei den Musiker( ${ }^{\star}$ inne)n mit Beeinträchtigung wurde von den klassischen Interessen von Musikschaffenden ausgegangen, die einerseits aufgrund der Vorgabe der Musik mit den bereits beschriebenen Interessenskonflikten einhergehen, andererseits Interessen wie Verdienst, Programmwahl, Konzertwünsche etc. nicht im höchsten Sinne erfüllt werden können. Ein weiterer Unterschied, der im direkten Verhältnis zum Professionalisierungsgrad steht, ist in der Kategorie der Verantwortung sichtbar. Die Partizipationsgrade der restlichen Kategorien unterscheiden sich nicht so gravierend, hier spielt der Kompetenzunterschied offensichtlich nur eine marginale Rolle. Die soziale Komponente, wie Schütz (1972) sie betont, sowie die Zweidimensionalität des Musizierens (sozialer sowie künstlerischer Prozess) nach Kloth (2016) kommt hier deutlich zum Ausdruck und sorgt für eine Ausgewogenheit innerhalb des musikalischen Geschehens.

Betreffend den Bereich der strukturellen Partizipation wäre ferner interessant, inwieweit die Öffnung der Strukturen unabhängig davon, welche Erwartungen den zugeschriebenen Rollen der einzelnen teilnehmenden Menschen innerhalb des Projektes zu Teil werden, unerwartete Fähigkeiten hervorbringen würde. Beispielsweise das Anvertrauen von administrativen Aufgaben, die 
bisher nur von den Entscheidungstragenden erfüllt werden. Dies wäre auch im Sinne eines Abbaus von sekundären Barrieren eine mögliche Strategie, neue Denkweisen zu etablieren.

\subsection{Maßnahmen zur Optimierung des Partizipationsgrades}

Aus den Diskussionen konnten einige Ideen herausgearbeitet werden, die vor allem im strukturellen Bereich einer Verbesserung der Partizipation und in weiterer Folge einem vertieften Verständnis von Inklusion dienen würden. Folgende Maßnahmen bieten sich an:

- Im Bereich der Programmauswahl werden die Musiker*innen noch weitestgehend ausgeschlossen. Hier empfiehlt es sich, aktiv Wünsche einzuholen, diese auf Tauglichkeit zu prüfen und wenn möglich umzusetzen. Sollten Liedwünsche abgelehnt werden, ist eine Begründung dafür zu geben. Für den Entscheidungsprozess wäre es wünschenswert, Vertreterinnen und Vertreter der Zielgruppe zu beteiligen (Steigerung des Mitspracherechtes, der Entscheidungskompetenz und der Informationsweitergabe (vgl. dazu Schwab, 2016)).

- Die Einbindung einer Vertreterin oder eines Vertreters der Zielgruppe in beratender Tätigkeit, beispielsweise bei den Treffen des Vereinsvorstandes, würde vor allem in der Kategorie der Mitsprache eine wesentliche Steigerung bedeuten. Eventuell wäre es sogar anzudenken, dieser Person ein aktives Entscheidungsrecht einzuräumen, um auch in diesem Bereich eine Steigerung der Partizipation zu erreichen. Möglicherweise wären entsprechende Unterstützungsmaßnahmen in Form von angemessenen Vorkehrungen, wie in der UN-BRK verankert, notwendig: „Zur Förderung der Gleichberechtigung und zur Beseitigung von Diskriminierung unternehmen die Vertragsstaaten alle geeigneten Schritte, um die Bereitstellung angemessener Vorkehrungen zu gewährleisten" (Art. 5, UN-BRK). Dies könnten Maßnahmen wie die Einbindung der leichten Sprache, Modelle unterstützter Entscheidungsfindung (vgl. Mayrhofer, 2013) oder andere unterstützende Hilfestellungen sein (siehe dazu auch Art. 4 (h), UN-BRK).

- Eine weitere Maßnahme kann die verstärkte Weitergabe von Informationen sein. Es wurde bereits die Möglichkeit angesprochen, Protokolle der Vereinssitzungen der Zielgruppe zugänglich zu machen, wobei hier Unterstützungsmöglichkeiten für die Informationsaufnahme notwendig erscheinen.

- Die Kategorie Verantwortung ist im Bereich der strukturellen Partizipation auf dem Stufenmodell relativ weit unten angesiedelt. Für diese Kategorie wurde in der Diskussion mit den Musiker( ${ }^{*}$ inne)n ein Impuls gesetzt. 
Beispielsweise wäre es denkbar, in einem ersten Schritt Musiker*innen mit Aufgaben wie der Abwicklung des Verkaufs von T-Shirts zu betrauen.

- Um dem Inklusionsanspruch des Projektes ein der UN-BRK angepasstes, zeitgemäßes Verständnis zu verleihen, wären Fortbildungs- bzw. Weiterbildungsangebote eine empfehlenswerte Maßnahme, um der Forderung der Behindertenrechtskonvention gerecht zu werden (Art. 8, UN-BRK).

- Der Ausbau der Inklusion für das Projekt kann auch dahingehend weitergedacht werden, dass verstärkt Bemühungen getätigt werden, um Musiker*innen ohne Beeinträchtigung zu gleichen Bedingungen für die Band zu gewinnen. Vor allem auch vor dem Hintergrund der Vermeidung eines Separationscharakters, dessen Ausprägung speziell den Bereich der Freizeit für Menschen mit Behinderung wesentlich einschränkt (vgl. Markowetz, 2016 und 2006).

- Weitere Maßnahmen, die der Optimierung der Partizipation dienen, sollten in partizipativen Arbeitsprozessen, idealerweise mit Beteiligungsmöglichkeiten für Musikerinnen, stattfinden.

\subsection{Ausblick}

Die erarbeiteten Ergebnisse dieser Studie beinhalten eine Ist-Stand-Analyse der Partizipationsfrage innerhalb der POWERband Tirol aus dem Jahre 2017. Die empfohlenen Maßnahmen wurden aus dem erhobenen Datenmaterial abgeleitet und dem Vorstand der POWERband Tirol vorgelegt. Ergänzend dazu wurden die Ergebnisse im Rahmen einer Konzertveranstaltung der POWERband Tirol einer breiten Öffentlichkeit zugänglich gemacht.

Einzelne Maßnahmen, wie beispielsweise die Liedwünsche der Musikerinnen einzuholen und in das Programm aufzunehmen, wurden bereits realisiert, weitere Maßnahmen stehen in Diskussion, wobei eine stetige Weiterentwicklung der Band als notwendiges Ziel definiert wurde. Dazu gab es im Frühjahr 2019 einen Konzepthalbtag, an dem über eine klarere Positionierung im Sinne der Inklusion diskutiert wurde und entsprechende Maßnahmen (Öffentlichkeitswirksamkeit, Begriffsschärfungen, musikalische Positionierungen, Überarbeitung der Webseite) dazu umgesetzt werden sollten. Der Prozess der partizipativen Evaluierung der POWERband Tirol hat somit wesentlich zur Schärfung eines Inklusionsbegriffes, wie er im Sinne der UN-BRK verstanden wird, beigetragen. Dadurch entstand innerhalb des Vorstandes ein neues Handlungsbewusstsein, welches anhand der geplanten und bereits umgesetzten Maßnahmen festgemacht werden kann. Ein zweiter Evaluationsprozess wäre eine spannende Maßnahme, um Fortschritte zu beschreiben, aber auch, 
um blinde Flecken zu identifizieren und daraus weitere Entwicklungsmöglichkeiten zu generieren. Vor allem darf durchaus mit einem kritischen Blick auf den Prozess geblickt und die Frage in den Raum gestellt werden, an welchen Stellen aufgrund eigener Betroffenheit noch Bereiche nicht sichtbar wurden. Sehr deutlich wurde der Einfluss persönlicher Interessen an der Stelle, an der es sich entschied, ob der Forschungsprozess auf eine Gesamtheit des Vereinsgeschehens oder auf Teilbereiche (Prozesse der Administration vs. musikalische Prozesse) anzuwenden ist. Die sich daraus entwickelte Diskussion barg durchaus ein gewisses Konfliktpotenzial, da die Vorstandsmitglieder unterschiedliche Positionen zu Bereichen des Vereinsgeschehen hatten. Vor allem dem musikalischen Leiter war die Unterscheidung zwischen administrativen und musikalischen Prozessen sehr wichtig. Aus dieser Diskussion konnte sich allerdings eine Richtung entwickeln, die sich als sehr vorteilhaft für den gesamten Forschungsprozess entpuppte und dazu diente, ein differenziertes Bewusstsein in Bezug auf Inklusion zu erarbeiten.

\section{Literatur}

Aichele, V. (2010). Behinderung und Menschenrechte: Die UN-Konvention über die Rechte von Menschen mit Behinderungen. Aus Politik und Zeitgeschichte, Nr. 23, 13-18.

Bergold, J. \& Thomas, S. (2012). Partizipative Forschungsmethoden: Ein methodischer Ansatz in Bewegung. Verfügbar unter: https://www.qualitative-research. net/index.php/fqs/article/view/1801/3333 [05.08.2020].

Flick, U. (2014). Qualitative Sozialforschung: eine Einführung (6. Auflage). Reinbek bei Hamburg: Rowohlt.

Flieger, P. (2017). Partizipation. In K. Ziemen (Hrsg.), Lexikon Inklusion (S. 179180). Göttingen: Vandenhoeck \& Ruprecht.

Greuter, S. (2017). Partizipation von Menschen mit Behinderung innerhalb eines inklusiven Musikensembles am Beispiel der POWERband TIROL. Unveröffentlichte Bachelorarbeit, Universität Innsbruck.

Hagen, J. (2002). Zur Befragung von Menschen mit einer geistigen oder mehrfachen Behinderung. Geistige Behinderung, 41 (4), 293-306.

Hinz, A. (2008). Inklusion - historische Entwicklungslinien und internationale Kontexte. In I. Körner \& U. Niehoff (Hrsg.), Von der Integration zur Inklusion. Grundlagen-Perspektiven-Praxis (S. 33-52). Marburg: Lebenshilfe-Verlag.

Kloth, A. (2016). Musizieren in inklusiven Settings. Eine qualitative Analyse am Beispiel der Band Just Fun der Musikschule Bochum. Verfügbar unter: https:// www.inklusion-online.net/index.php/inklusion-online/article/view/327/279 [05.08.2020]. 
Krebber-Steinberger, E. (2014). „All Inclusion!?“ - Teilhabe an der Musikkultur zwischen Verwirklichungschancen und Vermittlungsanspruch. Zeitschrift für Inklusion, 4. Verfügbar unter: https://www.inklusion-online.net/index.php/in klusion-online/article/view/244 [15.10.2020].

Kuckartz, U. (2014). Qualitative Inhaltsanalyse. Methoden, Praxis, Computerunterstützung (2. Auflage). Weinheim und Basel: Beltz Juventa.

Markowetz, R. (2006). Freizeit und Behinderung - Inklusion durch Freizeitassistenz. Spektrum Freizeit, 30 (2), 54-72.

Markowetz, R. (2016). Inklusion. In I. Hedderich, G. Biewer, J. Hollenweger \& R. Markowetz (Hrsg.), Handbuch Inklusion und Sonderpädagogik (S. 123-127). Bad Heilbrunn: Klinkhardt.

Mayrhofer, H. (2013). Modelle unterstützter Entscheidungsfindung. Beispiele guter Praxis aus Kanada und Schweden. IRKS Working Paper, 16.

Niediek, I. (2014). Auf die Frage kommt es an. Das Problemzentrierte Interview bei Menschen mit einer geistigen Behinderung. Teilhabe, 3, 100-105.

Schütz, A. (1972). Gemeinsam Musizieren. Die Studie einer sozialen Beziehung. In A. Brodersen (Hrsg.), Gesammelte Aufsätze (Band II: Studien zur soziologischen Theorie) (S. 129-150). Den Haag: Springer. https://doi.org/10.1007/978-94-0102849-3_7

Schwab, S. (2016). Partizipation. In I. Hedderich, G. Biewer, J. Hollenweger \& R. Markowetz (Hrsg.), Handbuch Inklusion und Sonderpädagogik (S. 127-131). Bad Heilbrunn: Klinkhardt.

von Unger, H. (2014). Partizipative Forschung. Einführung in die Forschungspraxis. Wiesbaden: Springer Verlag. https://doi.org/10.1007/978-3-658-01290-8

Wright, M. T., Block, M., Kilian, H. \& Lemmen, K. (2013). Förderung von Qualitätsentwicklung durch Partizipative Gesundheitsforschung. Prävention und Gesundheitsförderung, 3, 147-154.

Wright, M. T., Block, M. \& von Unger, H. (2008). Stufen der Partizipation. Verfügbar unter: https://www.sozialministerium.at/Themen/Soziales/Menschen-mitBehinderungen/UN-Behindertenrechtskonvention.html [05.08.2020]. https:// doi.org/10.1007/s11553-013-0396-Z 


\title{
Young Symphony - ein inklusives Education-Programm der Wiener Symphoniker
}

\author{
Don Quixote reloaded mit der Partnerschule \\ Neue Mittelschule Gassergasse
}

\section{Das Musikvermittlungsprogramm Young Symphony}

Die Wiener Symphoniker können zu den Spitzenorchestern des europäischen Musiklebens gezählt werden. Mit mehr als 160 jährlichen Auftritten und 128 festangestellten Musiker $\left({ }^{*}\right.$ inne)n decken sie ein breit gefächertes musikalisches Spektrum auf international höchstem Niveau ab. Schwerpunkt ihrer Konzerttätigkeit sind Abonnementreihen ${ }^{1}$ in den beiden großen Wiener Konzerthäusern, Wiener Konzerthaus und Musikverein. Darüber hinaus sind die Wiener Symphoniker seit 1946 Orchestra in Residence bei den Bregenzer Festspielen und eines der Hausorchester im Theater an der Wien. Konzerttourneen führen das Orchester regelmäßig in die wichtigsten Musikzentren der Welt, wie bspw. München, Paris, Tokio oder Zürich.

Als Orchester und offizieller Kulturbotschafter der Stadt bilden die Wiener Symphoniker mit ihren Konzerten, Übertragungen, Aufnahmen, innovativen Vermittlungsprojekten und Aktivitäten in den Wiener Gemeindebezirken das Rückgrat des Wiener Musiklebens und machen außerordentliche Musikerlebnisse allen Menschen zugänglich. Mit ihrem Musikvermittlungsprogramm Young Symphony richten sich die Wiener Symphoniker an ein Publikum jeder Altersgruppe und Herkunft: Das breitgefächerte Angebot reicht von Projekten mit mehrjähriger Laufzeit wie bspw. Schulpartnerschaften bis hin zu One-offs wie Probenbesuchen und Schul- oder Familienkonzerten, zu denen tausende Kinder kommen. ${ }^{2}$

Angeboten werden des Weiteren Musikworkshops für unterschiedliche Gruppen (z. B. Schulklassen, Kinder, Lehrlinge, Erwachsene, Kinderheimkinder etc.), Probenbesuche mit Rahmenprogrammen, Kooperationen mit der Musikschule Wien im Bereich der Nachwuchsförderung und dreijährige

1 Die Wiener Symphoniker bieten aktuell sechs Konzertzyklen im Wiener Konzerthaus und zwei im Musikverein Wien an. Das aktuelle Angebot findet sich unter www.wienersymphoniker.at [28.07.2020].

2 Jede Saison besuchen ca. 5.000 Schüler*innen die Schulkonzerte der Wiener Symphoniker. 
Schulpartnerschaften (pro Spielzeit mit je einer Wiener Schule; bisher entweder mit Neuen Mittelschulen oder Gymnasien).

Das Besondere am Musikvermittlungsprogramm Young Symphony ist, dass es sich nicht nur um ein Zusatzangebot für Kinder und Jugendliche handelt. Vielmehr bündelt sich in dem Programm die Idee einer Öffnung des Orchesters für alle Bevölkerungsgruppen, das zudem alle Arbeitsbereiche - von der Programmgestaltung bis hin zur Entwicklung neuer Konzertformate - umfasst. Diesem Wunsch nach gesellschaftlicher Relevanz und direktem Kontakt mit möglichst vielen Menschen ist auch die Genese der Initiative der sogenannten Grätzl-Konzerte ${ }^{3}$ geschuldet, bei denen das Orchester seine angestammten Spielorte im Herzen Wiens verlässt, um in ungewöhnlichen Spielstätten in den Wiener Gemeindebezirken Konzerte zu geben. ${ }^{4}$

Eine andere Facette stellen strategische Partnerschaften und Kooperationen mit anderen Institutionen wie den Wiener Konzerthäusern, dem Theater an der Wien, den renommierten Wiener Museen oder aber dem Tiergarten Schönbrunn dar. Hier greifen die unterschiedlichen Vermittlungsformate ineinander und es entstehen vernetze Lernorte. So gab es z. B. im Rahmen des Projektes Karneval der Tiere Musikworkshops mit Wiener Symphonikern im Elefantenhaus des Tiergartens Schönbrunn. Im Anschluss an diese Workshops erhielten die teilnehmenden Schüler*innen eine Führung durch den Tiergarten - passend zum Musikstück. Es wurden also in erster Linie Tiere besucht und kennengelernt, die auch im Stück von Camille Saint-Saëns vorkommen. Im Rahmen des Projektes Bilder einer Ausstellung fanden Musikworkshops in den Ateliers der Albertina statt und im Anschluss führten Museumspädagog $\left({ }^{*}\right.$ inn)en der Albertina die Klassen durch die Ausstellung - wiederum mit einem gelenkten Blickwinkel speziell auf russische Werke sowie Bilder, die inhaltlich zum Stück von Modest Mussorgsky passten. Alle Kinder erhielten während der Museumsführung Kopfhörer und hörten teilweise zeitgleich auch Musik des russischen Komponisten.

3 Als Grätzl werden in Wien Teile von Wohnbezirken bezeichnet. Ein Grätzl umfasst meist mehrere Häuserblöcke, jedoch gibt es für diese kleinsten städtischen Einheiten keine offiziellen Grenzziehungen. Ein Bezirksteil kann mehrere Grätzl umfassen.

4 Das waren bisher beispielsweise die SimmCity, Reaktor Hernals, F23 in Liesing oder die Expedithalle (die frühere Ankerbrot-Fabrik im zehnten Bezirk Wien-Favoriten). 


\section{Inklusive Projekte im Rahmen von Young Symphony}

In diesem Kontext können zwei Initiativen besonders hervorgehoben werden: Konzerte für und mit Menschen mit Fluchthintergrund sowie dreijährige Schulpartnerschaften.

\section{Konzerte für und mit Menschen mit Fluchthintergrund}

Seit längerem wurden und werden regelmäßig Menschen mit Fluchthintergrund in Generalproben der Wiener Symphoniker eingeladen. In der Saison 2015/2016 startete das Orchester innerhalb seines Musikvermittlungsprogramms zusätzlich mit Projekten für und mit Menschen mit Fluchthintergrund. Es wurden Konzerte in Flüchtlingsheimen gespielt, bei denen nicht nur Musiker*innen der Wiener Symphoniker, sondern auch Profimusiker*innen aus Ländern wie z. B. Syrien und Afghanistan spielten. Die Einbeziehung der Menschen mit Fluchthintergrund, die vor Ort wohnten, war ein weiterer wichtiger Aspekt dieses Konzertformats. So endete jedes Konzert mit gemeinsamem Singen, Tanzen und Essen.

\section{Das Konzertformat Ad.agio}

Welche Sprachen Ludwig van Beethoven wohl hörte, als er zu Beginn des 19. Jahrhunderts durch die Wiener Innenstadt spazierte? Ob er sich als Deutscher fühlte? Oder als Wiener mit Migrationshintergrund? Wohl eher als Weltbürger in einer kosmopolitischen Hauptstadt, welche die Vielfalt der Völker der Donaumonarchie als bereichernd empfand. Umso stimmiger erschien es dem Team der Wiener Symphoniker, Beethovens Musik auf syrische Musik treffen zu lassen. Das Konzertformat Ad.agio wurde vom Musiker und Musikvermittler Andrea Apostoli entwickelt und in unzähligen Konzerten in mehreren Ländern aufgeführt. ${ }^{5}$ Für das Wiener Projekt wurde er von den Wiener Symphonikern eingeladen und entwickelte mit diesen das Format noch weiter. So wurden nach einem Konzert im Flüchtlingsheim die dort lebenden Menschen anschließend als Zuhörer*innen ins Wiener Konzerthaus eingeladen, wo ein weiteres interkulturelles Konzert stattfand. So fand auf mindestens vier Ebenen eine Neuanordnung und Vermischung statt: Auf der Bühne, im Publikum, zwischen Publikum und Künstler( ${ }^{*}$ inne)n und auf programmatischer Ebene: Das Publikum war in der Mitte des Saales auf dem Boden platziert, die Musiker*innen musizierten rund um das Publikum sitzend. Das Villarmé

5 Mehr Informationen zu Andrea Apostoli findet man auf seiner Homepage: https:// www.musa-concerts.com/en/andrea-apostoli-en1.html [28.07.2020]. In Wien gab es eine Besonderheit, denn das Format wurde im Vorfeld des offiziellen Abendkonzertes im Wiener Konzerthaus auch im Haus Erdberg (Caritas Wien, Haus für Asylwerbende) gespielt. 
Quartett - bestehend aus Streicherinnen der Wiener Symphoniker - spielte gemeinsam mit Andrea Apostoli (Flöte) Musik von Ludwig van Beethoven. Abwechselnd dazu erklang Musik aus Syrien mit dem mittlerweile in Wien lebenden syrischen Musiker Salah Ammo sowie seinem Partner Peter Gabis. Den Abschluss bildete ein gemeinsam von den Wiener Musikerinnen und syrischen Musikern dargebotenes Stück - umgeben vom tanzenden Publikum.

\section{Dreijährige Schulpartnerschaften}

Innerhalb des Young-Symphony-Programms kommt es bei den Schulpartnerschaften zu besonders nachhaltigen Begegnungen zwischen den Orchestermusiker( ${ }^{*}$ inne)n und Kindern, die sonst vermutlich keinen Zugang zu klassischer Musik haben. Während drei aufeinanderfolgenden Jahren arbeiten die Wiener Symphoniker und eine Wiener Schule intensiv und vertieft zusammen, wobei sich die Schulen bislang immer aktiv beim Orchester meldeten. Bei der Auswahl der Schulen wird besonders darauf geachtet, dass solche gewählt werden, die an sozialen Brennpunkt-Standorten in Wien liegen und somit tatsächlich Kinder erreicht werden, die sonst wenig mit kulturellen Angeboten in Berührung kommen. Profimusiker*innen und Kinder sollen in dieser Zeit voneinander profitieren und sich bestmöglich kennenlernen, insbesondere durch zahlreiche Musikworkshops. Bei diesen stellen Musiker*innen der Wiener Symphoniker ihre Instrumente vor, die Schüler*innen können diese auch ausprobieren, es kommt zu langen, spannenden Gesprächen, und - besonders wichtig - zu aktiven, kreativen Übungen mit den Jugendlichen. Dabei wird von klassischen Werken ausgegangen, die die Schüler*innen in Folge in einem Konzert oder einer Probe hören. Wichtig ist, dass nicht kanonisches Wissen vermittelt werden soll, die vorbereitenden Übungen lassen vielmehr eine eigene kreative Herangehensweise der Jugendlichen $\mathrm{zu}$ - so wird sehr oft zu einem Thema improvisiert oder komponiert, Texte zur Musik werden erfunden oder Bilder gemalt. Höhepunkte der Partnerschaften sind gemeinsame Aufführungen oder eine aktive Beteiligung und Mitwirkung auf der Bühne durch Schüler*innen der Partnerschule an Schulkonzerten der Wiener Symphoniker.

Die Partnerschule seit dem Schuljahr 2018/19 ist die Neue Mittelschule Gassergasse aus dem fünften Wiener Gemeindebezirk. Sie liegt im Herzen von Wien zwischen Hauptbahnhof und Matzleinsdorferplatz. ${ }^{6}$ Seit dem Jahr 2018

6 Frau Andrea Walach, Direktorin dieser Mittelschule beschreibt ihre Schüler*innen in einem Gespräch sinngemäß folgendermaßen: „Viele unserer Kinder sind aus finanziell schwachen Familien. Ihre Eltern können häufig weder Deutsch sprechen noch verstehen und sind wenig (aus-)gebildet. An unserer Schule sind 98\% Kinder mit einer anderen Muttersprache, die meisten Eltern fallen als finanzielle Unterstützer und kulturelle Förderer für unsere Kinder leider weg. Alles, was die Schule nicht bietet, wird den Kindern somit auch nicht geboten. Deshalb ist die Zusammenarbeit mit den Wiener Symphonikern enorm wichtig, da unsere 
fanden bereits mehrere Projekte mit dieser Schule statt. Im Folgenden soll im Besonderen auf das erste größere Projekt eingegangen werden, Don Quixote reloaded.

\section{Das Schulprojekt Don Quixote reloaded}

Bei den sogenannten kreativen Projekten arbeiten im Rahmen von Schulpartnerschaften Musiker*innen der Wiener Symphoniker sowie auch Musikvermittler*innen und andere Künstler*innen über mehrere Wochen mit Jugendlichen rund um ein musikalisches Thema bzw. ein Werk der klassischen Orchesterliteratur zusammen. Ausgehend von dem Werk oder Thema erarbeiten die jungen Menschen sich dabei eigene Zugänge. Die vielschichtigen Ergebnisse werden schließlich bei öffentlichen Aufführungen vorgestellt. Das Projekt Don Quixote reloaded fand von März bis Mai 2018 statt, zwei Klassen der NMS Gassergasse (40 Schüler*innen aus den Klassen 1a und 2b, Alter: 10-12 Jahre) beteiligten sich am Projekt. Der Don-Quixote-Stoff wurde gewählt, weil er zusammen mit Jugendlichen in vielfältiger Weise bearbeitet werden kann. Zunächst lasen die Schüler*innen im Deutschunterricht die Geschichte und sahen sich einen leicht zugänglichen Kurzfilm mit Legomännchen über Don Quixote an. Anschließend stellten zwei externe Musikvermittlerinnen das gleichnamige Werk von Richard Strauß in Ausschnitten vor, mit dem Ziel, vielfältige Zugänge zur Musik zu schaffen. Danach fand ein Brainstorming zu Ideen der Schüler*innen für ihre eigene Don-Quixote-Aufführung statt. Es wurde die Idee geboren, dass eine neue Version der Geschichte erzählt werden und ein selbst verfasstes Textbuch für die Aufführung entstehen sollte (siehe Drehbuchauszug). Für die Umsetzung dieses Projektvorhabens wurden außerdem Stabpuppen gebastelt und gemeinsam mit Wiener Symphonikern wurden musikalische Improvisationen zu Themen aus Richard Strauss' Tondichtung Don Quixote erarbeitet. Welche Themen verwendet wurden, bestimmten die Schüler*innen. Der Schulchor studierte zudem den Song I Have a Dream der Gruppe ABBA ein. Am 29.05.2018 fand im Bezirksamt Margare-

Schüler*innen nur über die Schule die Gelegenheit erhalten, einmal in ein Konzerthaus zu kommen, Musikerinnen in ihrem Klassenraum begrüßen zu dürfen und in eine andere Welt einzutauchen. Die zusätzliche professionelle Mitwirkung und Arbeit der Wiener Symphoniker stellt also ein besonderes Highlight für unsere Kinder und die Schule im Gesamten dar." 
ten die erfolgreiche Abschlussaufführung von Don Quixote reloaded statt, bei der auch sechs Musiker*innen der Wiener Symphoniker mitwirkten. ${ }^{7}$

Diese spielten in der Aufführung Ausschnitte aus Richard Strauss' Don Quixote, Fantastische Variationen über ein Thema ritterlichen Charakters - eine Tondichtung für großes Orchester op. $35 \operatorname{TrV} 184$ in einem Arrangement von Friedrich Philipp-Pesendorfer. Die Schulpartnerschaft wurde im Rahmen des Programms Blick_Wechsel - Entwicklungsprozesse mit kultureller Bildung für Pflichtschulen durchgeführt und von KulturKontakt Austria unterstützt.

Abschließend ein Drehbuchauszug der Aufführung, verfasst von Schüler ( ${ }^{*}$ inne)n der NMS Gassergasse:

\section{SZENE 1/ Treffen von zwei Freundinnen}

Begrüßung: Hallo, wie geht's?

1: Du, ich habe ein Spiel gefunden, das du sicher noch nicht kennst!

2: Was? Ein neues Spiel? Geil!

1: Ich glaube es ist schon ziemlich alt.

2: Wie heißt es?

1: Die Abenteuer von Don Quijote.

2: Komischer Name

1: Hast ja recht, lass es uns trotzdem ausprobieren!

Betätigen der Playstation / Symphoniker: Musik von Tetris

2: Hey. Das ist das falsche Spiel, das ist doch Tetris!

1: Hau ab damit, das spielen doch nur mehr Opas und Omas!

2: Da, aber jetzt hab ich es! Es geht los!

Playstation - Stimme aus dem Off

„Herzlich Willkommen bei den verrückten Abenteuern von Don Quijote! Don Quijote war ein armer Landedelmann. Er lebte vor langer, langer Zeit und war verrückt nach Ritterromanen. Seine gesamte Zeit verbrachte er damit, bis er eines Tages beschloss, selbst Ritter zu werden und die Menschen gegen alle bösen Mächte zu verteidigen.“

7 Musiker*innen: Stefan Pöchhacker (Violine), Manuel Gangl (Klarinette), Judith McGregor (Viola), Zsofia Günther-Mészáros (Violoncello), Hans-Joachim Tinnefeld (Kontrabass), Reinhard Hofbauer (Posaune), Friedrich Philipp-Pesendorfer (Vibraphon und Arrangements). Musikvermittlerinnen: Judith McGregor, Iris Winter, Bettina Büttner-Krammer (Projektleitung). 
Symphoniker: Thema Don Quijote

Ziel des Spieles ist es, die Welt zu retten!

LEVEL 1: Du brauchst 1 Pferd, 1 Lanze,1 Rüstung, einen Helm, einen Knappen und einen Esel für deinen Diener. Findest du diese Dinge, erst dann kannst du den Ritterschlag empfangen.

\section{Auftritt Don Quijote}

Zur Aufführung kamen nicht nur Eltern und Freunde, sondern auch die gesamte Schule. Ausschnitte aus dem Projekt gibt es in drei kurzen Videos auf youtube:

Video 1: https://www.youtube.com/watch?v=HPP4_9YSt50

Video 2: https://www.youtube.com/watch?v=ad9VoKyMp30

Video 3: https://www.youtube.com/watch?v=dh2A7hi5QKg 



\section{Alles ganz normal!?}

\section{Franziska Lottner erhält einen 1. Preis bei Jugend musiziert}

Völlig routiniert geht sie die Treppe der Schule hinauf. Gleich wird sie ihr Programm mit ihrer Begleiterin noch ein letztes Mal im Schnelldurchlauf singen. Es ist der 27. Januar 2018. Der alljährliche spannende Tag des Jugend-musiziert-Regionalwettbewerbes ist endlich da. Franziska nimmt bereits zum dritten Mal teil. Die ersten beiden Male hat sie jeweils einen 3. Preis ergattert. Für Franziska war das alles andere als zufriedenstellend. Dennoch lässt sie sich nicht entmutigen und geht dem Wettbewerb erneut mit frohem Mut und vollem Einsatz entgegen. Mit der Planung und ihrer Programmauswahl begann sie bereits ein Jahr zuvor.

An diesem Wettbewerbstag ist es viel entspannter als in den vorigen Jahren. Man merkt ihr die Erfahrung an, die sie im vergangenen Jahrzehnt gewonnen hat.

Nach einer angenehmen Anspielprobe begibt sie sich in die Startlöcher und wartet hinter der Bühne, bis sie abgeholt und auf ihren Stuhl begleitet wird. Ihre Musicalszene beginnt sie damit, sich vorzustellen. „Mein Name ist Franziska Lottner, ich bin 23 Jahre alt und arbeite in der Behindertenwerkstätte in Sulzbach-Rosenberg. Ich habe einen angeborenen Hydrocephalus und bin gehbehindert. "1 Ohne es wirklich zu bemerken, befinden sich die Jury und das Publikum mitten in der schauspielerischen Darbietung. Franziska betont, wie normal ihr Leben ist und dass sie wie jede und jeder andere auf der Welt lernen, besser werden und ihre Ziele erreichen kann. Das beweist sie, indem sie nun nach neun Jahren Gesangsunterricht heute sogar ihr selbst komponiertes Lied vorträgt. Mit heller, klarer Stimme und einem Lächeln auf dem Gesicht singt sie: „Jeder Mensch ist was Besond'res, ganz egal wie er so ist“.

Franziska erzählt dann weiter von ihren Träumen, so tanzen zu können wie alle anderen, aber leider schafft sie es nicht, alleine aufzustehen. Ihre ganze Entschlossenheit, trotzdem nicht aufzugeben, legt sie in das Stück Ich bin bereit aus dem Film Vaiana. Sie möchte an Wunder glauben und daran, dass sie hier und jetzt geheilt werden kann, erklärt Franziska nach dem Song. Mit Superkalifragilistikexpialigetisch hat sie vielleicht ein magisches Wort gefunden, das sie gesund zaubern kann. Nachdem der letzte Ton des Mary Poppins Liedes verklungen ist, versucht sie aufzustehen, fällt aber wieder zurück in ihren Stuhl.

1 Alle Zitate und Beschreibungen von und über Franziska Lottner haben Ihre persönliche, freundliche Genehmigung gefunden. 
Und immer noch gibt Franziska nicht auf: „Es liegt bestimmt daran, dass ich Umhang und Zauberstab vergessen habe“, ruft sie, schnappt sich ihre Requisiten und malt nun schöne, choreografierte Formen punktgenau zur Instrumentalbegleitung ihres Zauberspruchs in die Luft. Nochmal versucht sie aufzustehen. Es klappt wieder nicht. Nach dem dritten Versuch gibt sie auf und singt einfühlsam You must love me aus Evita.

Franziska bekommt einen besonders lauten und langen Applaus, den sie freudestrahlend mit mehreren Verbeugungen entgegennimmt, solange, bis sie wieder von der Bühne abgeholt wird. Die Jury berät nicht lange und verkündet das Ergebnis im Wettbewerbs-Café. Franziska hat einen 1. Preis! Mit einem spitzen und sehr langen Freudenschrei schmeißt sie die Arme in die Luft und ruft: „Was für ein Schock. Ich kann es nicht glauben! Das dauert jetzt sicher vier Tage, bis ich das verarbeitet habe." Alle stehen applaudierend und überglücklich um die Preisträgerin herum. Mama, Papa und ihre Lehrerin haben Tränen in den Augen und umarmen sich gegenseitig. Franziska hat ihren Traum wahr werden lassen. Im Jurygespräch bekommt sie große Anerkennung: „Das war die berührendste Darbietung des heutigen Tages.“ Wow! Was für ein Lob, und welch großes Ereignis in ihrem Leben und ohne große Öffentlichkeit. Franziska hat es geschafft, trotz Handicap mit allen anderen mitzuhalten und sie sogar zum Teil noch zu überbieten. Von alles ganz normal kann heute absolut nicht die Rede sein.

\section{Der Schlüssel zu Franziskas Erfolg}

Was war der Schlüssel zu Franziskas Erfolg? Sicherlich zu einem großen Teil die Selbstverständlichkeit, die sich nach einem Jahrzehnt voller Konzerte und Wettbewerbe bei ihr eingestellt hat. Im Laufe ihres Gesangsunterrichts, der von 2009 bis 2018 als Einzelunterricht stattfindet, hat Franziska an vielen unterschiedlichen Projekten teilgenommen: an Castings, Musicalaufführungen Szenenabenden, Konzerten mit und ohne Begleitung, Jugend musiziert Wettbewerben, Chorauftritten mit und ohne Solo, einer CD-Aufnahme uvm. Zudem fertigte sie im Lauf der Zeit eigene Kompositionen an. Eine davon wurde als Musikvideo verfilmt, in dem sie die Hauptrolle spielte. Außerdem gab es über sie einen Bericht mit Interview im regionalen Fernsehen.

In ihrem 45-minütigen Einzelunterricht und dem 45-minütigen Dreiergruppenunterricht arbeitet sie gleichzeitig an einigen Stücken (Stand: Oktober 2018): 
- vier Duo-Stücke für Jugend musiziert

- ein dreistimmiges Weihnachtslied

- eine Musicalszene als Terzett

- zwei mehrstimmige Stücke für das Faschingskonzert

Franziska ist in ihrer Heimatmusikschule eine tragende Säule. Seit Beginn ihres Unterrichts hat sie immer schon viel zu tun: Mit den zahlreichen Auftritten in unterschiedlichen Besetzungen und an verschiedenen Orten hat sie Bühnenfestigkeit erworben. Sie bestreitet Konzerte als normalen und spannenden Teil ihres Alltags. Eigentlich sind unvorhersehbare Dinge für Franziska aber eher irritierend. Wenn sie aufgeregt ist, dann sehr. Sie selber sagt über sich: „Ich mag geregelte Abläufe, deswegen übe ich seit Jahren jede Woche genau fünf Mal." Aber mit der Zeit fand sie einen Umgang mit der komplexen Bühnensituation, der sie mittlerweile gelassen begegnen kann.

Ein anderes Kriterium für ihren Erfolg ist vermutlich die Authentizität, mit der Franziska das Publikum verzaubert hat. Sich anderen zu zeigen, wie man ist, ist für Schauspielerinnen und Schauspieler ein langer Weg. Franziska hatte Spaß an ihrer Szene. Sie war ganz darin vertieft und konnte die Jury mitnehmen, die sichtbar und hörbar mit Lachen und Applaus darauf reagierte. Es sah so aus, als hätten sie auch Spaß. Für Musizierende ist die Herausforderung, sich und andere zu bewegen, wohl ständig präsent und es führt zu Frustrationen, wenn dies misslingt. Insofern erinnert die einleitende Geschichte alle Bühnenkünstlerinnen und -künstler daran, dass musikalische Spitzenleistungen mit der Natürlichkeit des Momentes verbunden sind. Auch für Franziska ist das kein Zustand, der ihr besonders leicht fällt. Die Jahre zuvor war sie so mit der Aufregung beschäftigt, dass sie oft ganz vergessen hatte, ihren Mund weit genug aufzumachen oder tief und ruhig zu atmen, wenn sie sang.

Mit Sicherheit lässt sich aber sagen, dass Franziskas Unermüdlichkeit eine notwendige Voraussetzung für ihren Erfolg war. Denn hätte sie nach zwei dritten Preisen bei Jugend musiziert, die sie selbst als große Niederlage empfunden hat, einfach aufgegeben, hätte sie ihren Wettbewerbs-Glückstag niemals erleben können. Franziska wollte unbedingt erfolgreich sein. Sie nimmt ihr Hobby ernst und möchte gefordert, gesehen und anerkannt werden.

Alle drei Erfolgskriterien können nicht in speziellen Unterrichtsformen und -inhalten verortet werden, die gezielt für Schülerinnen und Schüler mit Handicap aufbereitet sind. Es ist eher zu vermuten, dass Franziska über die Zeit hinweg gelernt hat, was sie für einen ersten Preis bei Jugend musiziert benötigt. Also doch wieder alles ganz normal, wie bei jedem anderen auch? 


\section{Ganz schön mutig}

„Ich liebe es, auf der Bühne zu zeigen, was ich kann.“, sagt Franziska über sich. Deswegen war es für sie immer selbstverständlich, alle möglichen Herausforderungen anzunehmen. Eine gute Voraussetzung, aber trotzdem ganz schön mutig. Und auch ich als Lehrerin musste diesen Mut aufbringen, meine Schülerin ins Ungewisse laufen zu lassen. Sie von einer Jury bewerten und beraten zu lassen, die sich auf diese Situation nicht vorbereitet hatte, ihre Beeinträchtigungen nicht kannte und somit nicht einschätzen konnte, wann sie für sich gesehen herausragende Leistungen erbringt. Selbst am Vorabend des Wettbewerbserfolgs fehlte Franziska und mir jegliche Einschätzung, was am nächsten Tag passieren würde. Wir wussten, dass die Szene gut ausgearbeitet und etwas Besonderes geworden war, aber wegen des fehlenden Erwartungshorizonts des Wettbewerbs gegenüber Musizierender mit Handicap konnten wir keinerlei Einschätzungen treffen. Sich in eine solche Ungewissheit zu begeben, gerade wenn man ein Handicap mit beispielsweise emotionalem Schwerpunkt hat, kann herausfordernd bis unmöglich sein. Zumal man als Lehrkraft die Verantwortung übernehmen muss einer Schülerin oder einem Schüler dieses hohe Risiko und eine mögliche und unvorhersehbar große Frustration zuzumuten.

Vermutlich ist das einer der Gründe, warum Franziska mit ihrem Handicap bei Jugend musiziert ein Einzelfall bleibt. Beim Bundesausschuss Jugend musiziert nachgefragt, bekommt man leider keine Statistiken über Teilnehmende mit Handicap. Es existieren keine Zahlen. Es gäbe Einzelfälle. Niemand würde explizit ausgeschlossen und alle seien willkommen, die die vorgegebenen Kriterien erfüllen können. Das erfahre ich in einem Telefonat. Aber genauso fällt der Satz: „Diese Gruppe kommt nicht vor“ (Pressestelle Jugend musiziert, 2018). Gemeint ist wohl, dass es bislang keine Kategorie gibt, die explizit für Menschen mit Behinderung ausgeschrieben wird.

Nachvollziehbare Daten erhalte ich vom Regionalausschuss Oberpfalz Mitte/Nord. Das ist Franziskas Heimatbezirk. Hier ist ihre Duoanmeldung im Folgejahr die einzige von insgesamt 57 Anmeldungen zum 56. Regionalwettbewerb, die von Menschen mit Beeinträchtigungen eingereicht wurde (vgl. Faderl, 2018). In den Jahren zuvor sah die Verteilung ähnlich aus. Franziska ist weit und breit die einzige Person mit Behinderung, die in dieser Region an Jugend musiziert teilnimmt. Insgesamt werden an Franziskas Musikschule in Sulzbach-Rosenberg im Schuljahr 2018/19 34 Menschen mit Handicap unterrichtet, während die Schule eine Gesamtschülerzahl von 1.173 Schülerinnen 
und Schülern aufweist. Menschen mit Beeinträchtigungen machen 0,35 \% des Gesamtvolumens aus. ${ }^{2}$

Ein Blick auf die deutsche Musikschullandschaft im Kalenderjahr 2016 zeigt eine stolze Schüler*innenzahl von 1.451.496 (vgl. VdM, 2017, S. 12-13). Die Anzahl der Schüler*innen mit Behinderung beträgt im Jahr 2016 deutschlandweit 13.532 (vgl. VdM, 2017, S. 24). Das entspricht einem Anteil von Menschen mit Beeinträchtigungen von weniger als $1 \%$.

\section{Paramusik-Wettbewerbe}

Anhand der Zahlen lässt sich kein Bedarf ableiten, wie viele Musikschülerinnen und -schüler mit Handicap an einem Wettbewerb teilnehmen würden. Jedoch lässt sich erkennen, dass es genügend Einzelfälle gibt, über die es sich lohnt nachzudenken. Viel wichtiger aber als eine statistische Notwendigkeit für Paramusik-Wettbewerbe ist das Potenzial, das für alle Beteiligten darin verborgen liegen könnte.

Durch die Bereitstellung solcher Paramusik-Wettbewerbe, die den Teilnehmenden einen sicheren Rahmen geben würden, als Lernumgebung für Menschen mit Handicap funktionieren und Motivation für die eigene Weiterentwicklung schaffen könnte, könnte ein enormes Potenzial für den gesamten Wettbewerbsbetrieb freigesetzt werden. Die Fragen, die man sich stellen muss, um Musizierenden mit Handicap gerechter werden zu können und ihnen einen genauso sicheren Platz im Wettbewerb zu geben, wie allen anderen auch, sind Fragen, welche die gesamte Bewertungsperspektive von Jugend musiziert bereichern könnten. Es handelt sich um Fragen nach Musik, die berührt und ansteckt und nach Menschen, die sie erklingen lassen, beispielsweise Fragen wie:

- Ab wann fühle ich mich als Publikum involviert? Was interessiert und berührt mich?

- Was macht eine gute Performance aus, jenseits von technischer Perfektion?

- Genügt es für einen gelungenen Vortrag, sich zu zeigen, wie man ist? Und wenn ja, was zeigt man denn da genau?

Handicaps können sehr unterschiedlich und vielfältig sein. Menschen mit Behinderung können sinnvollerweise nur als Einzelfall betrachtet werden. Ein Vergleich ist so gut wie unmöglich, da eine Sprachbehinderung im Gesangswettbewerb ganz anders bewertet werden muss, als beispielsweise eine Sehbehinderung. Mit der Möglichkeit, dass es unendlich viele gleiche Preise ge-

2 Diese Angaben entstammen meiner E-Mail-Korrespondenz mit dem Musikschulleiter Steffen Weber am 11. Dezember 2018. 
ben kann, versucht Jugend musiziert dem Anspruch einer Einzelfallbehandlung auch im bisherigen Wettbewerbsverlauf nachzukommen. Aber geschieht das auch wirklich? Stehen die Musizierenden für sich? Wird sie oder er individuell wahrgenommen oder entstehen automatisch Vergleiche mit den restlichen Beiträgen? Ich behaupte, dass Vergleiche immer stattfinden, einfach deshalb, weil wir gerne Zusammenhänge bilden, gerecht sein wollen und schlichtweg keine Option haben, sie wegzulassen. Und mal ganz ehrlich: Wir als Jurymitglied sind eigentlich auch heilfroh darüber, vergleichen zu können, um einen Anhaltspunkt zu bekommen. Vergleiche zu ziehen ist an sich auch nichts Schlechtes. Es kann die Trainingsspannung sowie die Fairness fördern und sorgt für ein möglichst hohes Grundniveau. Aber Vergleiche sind immer auch eine Gratwanderung. Am Ende ist es unsere Einzigartigkeit, die einen besonderen musikalischen Moment erzeugt. Technische Brillanz ist ein Werkzeug, um zu diesem Moment zu gelangen. Sie ist aber keine Voraussetzung. Kinder, die ihre Lieder mit fehlerhafter Aussprache singen, sind unmittelbar und anrührend. Eine gute Performance, die nicht langweilt, sondern überrascht, kann faszinierend sein. Die richtigen Stücke, gut und passend ausgewählt, können Räume für das Publikum und auch für Musizierende öffnen.

Wir sind unglaublich gut darin geworden, Technik zu beurteilen. Über sie können wir im Detail diskutieren. Aber können wir auch über die anderen Faktoren, die einen musikalischen Vortrag auszeichnen, adäquat reflektieren? Und damit meine ich nicht die Intuition, die jedem gegeben ist, Präsenz, Ausdruck und die Gesamtperformance zu beurteilen. Ich frage an dieser Stelle nach der gleichen Klarheit, Wichtigkeit und Tiefe, die wir auch in einer Diskussion über Perfektion und Technik erreichen können! Gelingt es uns, 20 Facetten zu Präsenz in der gleichen Eloquenz zu nennen wie 20 Facetten zu technischer Brillanz? Ist unser Grundverständnis zu Präsenz oder Ausdruck ähnlich, sodass eine gemeinsame Diskussionsbasis vorhanden ist? Ab wann driften wir in Bereiche des Geschmacks ab und die nachvollziehbaren Argumente gehen uns aus oder wollen nicht mehr zugelassen werden?

Wenn man den Lehrplan für Gesang des Verbandes deutscher Musikschulen studiert, entsteht der Eindruck, dass es hier tatsächlich an Detailwahrnehmung fehlt, zumal sie darin von den Lehrkräften auch nicht gefordert wird. Beispielsweise steht im zweiten Kapitel zum Instrument Stimme auf zwei DIN-A5-Seiten viel über die Entwicklung einer gesunden Stimmfunktion und den "Mechanismus zur Tongebung nach feststehenden Naturgesetzen“ (VdM, 1990, S. 12). Das „unsichtbare Ich, Geist und Seele, die Persönlichkeit des Menschen" wird zwar erwähnt, aber es werden keine Komponenten davon aufgedeckt oder in Bezug zu einem erfolgreichen musikalischen Vortrag gesetzt. Stattdessen steht nur ein einsamer (Ab-)Satz, der Performance, Ausdruck und Präsenz am Rande streift: 
„Bei der Entwicklung der sängerischen Persönlichkeit ist es unerläßlich [sic!], die Ganzheit des Menschen zu entfalten. Anzustreben ist eine individuelle Ausgewogenheit von Intellekt, Psyche und Physis.“ (VdM, 1990, S. 13)

Auch in den Unterrichtsinhalten für die Unterstufe im Gesangsunterricht finden sich nur wenige Punkte, die außertechnische Fähigkeiten einfordern, wie beispielsweise „Entwicklung von Konzentrationsfähigkeit und Phantasie“ und „Hinweise zu Auftrittsverhalten“(VdM, 1990, S. 18-19).

Eine differenziertere und auch detailreichere Auflistung liefert der deutlich modernere VdM Lehrplan für Querflöte. Statt drei Seiten nimmt der Unterrichtsplan hier elf Seiten ein und listet in tabellarischer Form vier verschiedene Lernkategorien für jede Niveaustufe, sowie ihre Lernziele, Lerninhalte und Methodiken auf. Eine der Kategorien heißt Musizieren. Hier lassen sich differenzierte Kriterien finden: Auf „Fähigkeit zur musikalischen Gestaltung entwickeln“ in der ersten Unterstufe folgt in der zweiten Unterstufe „Musikalisches Gestalten/Phrasierung“. In der ersten Mittelstufe findet man Formulierungen wie „Ausdrucksentwicklung und Stilsicherheit“ / „Zu individueller musikalischer Darstellung finden“ und „Auftrittstraining: Selbstbewusstes Auftreten mit angemessener Haltung und Ausstrahlung nach außen, engagiertes und mitreißendes Spiel durch körperliche Ausdruckskraft" (VdM, 2011, S. 36-44). Für die Mittelstufe 2 finden sich Umschreibungen wie „ausdrucksvoll“ oder "ansprechende Interpretation“, die hier stets in Verbindung mit anderen Anforderungen, wie beispielsweise Zusammenspiel, Stilsicherheit und Fehlerfreiheit stehen (vgl. VdM, 2011, S. 45), während die Lernziele für die Oberstufe hier nur „Interpretationsvergleiche“ (VdM, 2011, S. 47) anführen.

Die 21 Jahre, die zwischen beiden Lehrplänen liegen, sind merklich. Es zeigt sich eine Entwicklung hin zu genaueren Beschreibungen außertechnischer Komponenten für einen erfolgreichen Vortrag. Wir sind also vermutlich ohnehin dabei, die Perspektiven zu Ausdruck, Präsenz und Performance mehr auszuleuchten. Und genau hierfür kann die Auseinandersetzung mit Menschen mit Handicap eine weitere Hilfe sein. Menschen ohne Handicap können lernen, Menschen mit Beeinträchtigung holistisch zu verstehen, sich einzufühlen und den ganzen Menschen in seiner individuellen Situation zu sehen. Eine Beschäftigung mit dem Einzelfall schenkt diese Lupe, die hineinzoomt und Antworten gibt auf die Frage nach weiteren Faktoren, jenseits spieltechnischer Details, die zum Gelingen eines Vortrages beitragen. Eine Person unabhängig von anderen zu betrachten und ihr einen sicheren Raum geben zu können, erfordert fachliche und eventuell medizinische Kompetenz, aber vor allem Empathie und Mitgefühl. 
Wenn der Weg gegangen und ausreichend Verständnis gegenüber Menschen mit Handicap aufgebaut wurde, kann dies auf die Bewertung jeder musizierenden Person zurückwirken. Vielleicht könnte dadurch ein stärkeres Gleichgewicht erreicht werden zwischen Perfektion und Magie. Oder, um es abschließend mit Franziskas Worten zu formulieren:

"Ich habe mich gezeigt, wie ich bin, und hatte Erfolg. Wenn das bei mir klappt, dann doch wohl bei allen anderen auch, oder?"

\section{Literatur}

Faderl, G. (2018). Protokoll über die Sitzung des Regionalausschusses „Jugend musiziert" am 21.11.2018. Sulzbach-Rosenberg: Städtische Sing- und Musikschule.

Pressestelle "Jugend musiziert" (2018). Telefonat über gehandicapte Teilnehmende am Wettbewerb Jugend musiziert am 11.12.2018. München: Deutscher Musikrat gGmbH.

Verband deutscher Musikschulen e.V. (Hrsg.). (1990). Lehrplan Gesang. Regensburg: Bosse.

Verband deutscher Musikschulen e.V. (Hrsg.). (2011). Lehrplan Querflöte. Kassel: Bosse.

Verband deutscher Musikschulen e.V. (Hrsg.). (2017). Statistisches Jahrbuch der Musikschulen in Deutschland 2017. Bonn: VdM Verlag. 
Christine Schönherr

\section{„Man spürt, dass man lebt“}

\section{Musik-Sprache-Bewegung/Tanz}

\section{Künstlerisch-geragogische Angebote für Menschen in hohem Alter}

\section{Einführung}

Zeitungsüberschriften wie Zufriedenes Alter ist lernbar ${ }^{1}$, Altern tut nicht weh ${ }^{2}$, Altern in guter Gesellschaft ${ }^{3}$ und Hundertjährige wollen gern weiterleben ${ }^{4}$ machen deutlich, dass Alter und Altern brisante Themen unserer Zeit sind, die bewegen und über die nicht mehr hinweggeschaut werden kann. Das hängt meiner Meinung nach wesentlich mit der demographischen Entwicklung unserer Gesellschaft zusammen, mit der steigenden Lebenserwartung und dem gleichzeitigen Geburtenrückgang. Neben der Möglichkeit, damit einhergehende Potenziale zu leben, wie Interessensgebiete zu vertiefen, Kompetenzen zu steigern, in neue Bereiche einzusteigen, bisher unbekannte Ressourcen aufzuspüren und zu nutzen, treten auch viele Fragen auf. Da gibt es z. B. verständliche Wünsche der Älteren, in Gesundheit zu altern, geistig und körperlich rüstig zu bleiben, nicht in den Ruhestand gedrängt zu werden und die verlängerte Lebenszeit sinnerfüllt $\mathrm{zu}$ verbringen. Dazu möchte der nachfolgende Artikel Überlegungen anbieten, Erfahrungen mitteilen und Anregungen geben.

\subsection{Altersbilder und Alterstheorien}

Altersbilder spiegeln die vorherrschenden Einstellungen in den verschiedenen Epochen abendländischer Kultur wider, wie sie für diese Altersgruppe entwickelt wurden. Sie reichen vom Altersspott und der Altenaussetzung bis zu Verehrung und Idealisierung (vgl. Metz \& Pauls, 2004, S. 110). „Durch die Art, wie sich eine Gesellschaft gegenüber ihren Alten verhält, enthüllt sie unmiss-

1 Dieser Artikel findet sich in der Ausgabe der Salzburger Nachrichten, 21. Juni 2003 (Wochenende-Teil, S. 1).

2 Dieser Artikel findet sich in der Ausgabe der Salzburger Nachrichten, 19. Mai 2012 (Wochenende-Teil, S. 7).

3 Dieser Artikel findet sich in der Ausgabe der Salzburger Nachrichten, 3. Oktober 2009 (Lokal-Teil, S. 19).

4 Dieser Artikel findet sich in der Ausgabe der Salzburger Nachrichten, 20. Juli 2013 (Stammausgabe, S. 20). 
verständlich die Wahrheit - oft verschleiert - über ihre Grundsätze und Ziele“ (Metz \& Pauls, 2004, S. 110). Die Ambivalenz der Altersbilder reicht bis in die heutige Zeit. So haben sich seit den 1950er Jahren verschiedene Alterstheorien entwickelt. Zu Beginn stand das Defizitmodell. Es gilt in der Gerontologie inzwischen als widerlegt. Dem folgten die Aktivitätstheorie (Tartler, 1961) sowie ihr Gegensatz, die Disengagement-Theorie (Cumming \& Henry, 1961), die das Alter als allgemeinen Abbauprozess, gekoppelt mit freiwilligem Rückzug aus sozialen Bezügen beschreibt. Als Gegenentwurf sieht sich die Kompetenztheorie (Olbrich, 1987) sowie das Modell Erfolgreiches Alter(n) (Baltes \& Baltes, 1987), wobei ich das Adjektiv erfolgreich gerne durch erfüllt ersetzen möchte (vgl. Hartogh, 2008, S. 26-32). Rüdiger Dahlke betitelt sein im Oktober 2018 erschienenes Buch sogar mit Das Alter als Geschenk.

Die Alterstheorien wirken in alle Bereiche des gesellschaftlichen Lebens hinein. Sie können somit auch direkt oder indirekt bildungsorientierte Angebote für betagte Menschen beflügeln oder beeinträchtigen. Hilarion Petzold spricht diesbezüglich vom Feind von außen, der das Jungsein idealisiert und die Anti-Aging-Bewegung ankurbelt, sowie vom Feind von innen, der den alten Menschen denken lässt, dass er alt sei und nichts mehr könne (vgl. Petzold \& Berger, 1974, S. 398). Solche Einstellungen und Denkweisen sind kontrovers zu dem Anliegen, ein sinnerfülltes Alter zu leben. Dem Feind von innen und von außen versuchen wir im Rahmen unseres Musik- und Bewegungsangebots u. a. durch Übungen zu begegnen, in denen wir uns liebevoll und wertschätzend unserem Körper zuwenden, auch wenn er sich durch das Alter verändert hat, gebrechlich und steif geworden ist. Es ist zu beobachten, dass die Pluralisierung von Lebensstilen die Rollenbilder des Alters langsam aufbricht und erkannt wird, dass es nicht die homogene Gruppe der Alten gibt, sondern Menschen unterschiedlich altern, und dass das kalendarische Alter keineswegs immer dem biologischen entspricht.

\subsection{Wiesbadener Erklärung des Deutschen Musikrates}

Die Überzeugung, dass Musik wesentlich dazu beiträgt, kreative Potenziale älterer Menschen zu entfalten, und diese in die Gesellschaft eingebracht werden können, hat zu der sogenannten Wiesbadener Erklärung geführt. Sie enthält zwölf Forderungen an Politik und Gesellschaft, in denen es u.a. heißt: „Mit dem Bild einer human orientierten Gesellschaft verbindet sich die Überzeugung, dass die Erfahrung mit Musik um ihrer selbst willen als elementarer Bestandteil in jedem Lebensalter ermöglicht werden muss." (Hartogh \& Wickel, 2008, S. 147). Das Jahr 2012 wurde dann vom Europäischen Parlament und dem Europarat zum Europäischen Jahr des Aktiven Alters und der Solidarität 
zwischen den Generationen erklärt, mit der Absicht, für die Chancen und Probleme der demografischen Entwicklung unserer Gesellschaft zu sensibilisieren.

\section{Grundsätzliches zu Bildungs- und Musikangeboten für ältere Menschen}

Bildungsangebote auf breiter Basis können wesentlich dazu beitragen, dass die verlängerte Lebensspanne möglichst selbstbestimmt und sinnerfüllt verbracht, vorhandene Kompetenzen genutzt und Defiziten, die das Alter mit sich bringt, begegnet werden kann. Sie sollten an Hobbys und kulturelle Interessen der Menschen anknüpfen, ebenso einen Neueinstieg ermöglichen und auf verschiedene Weise in Bezug stehen zu folgender Vier-L-Alters-Zauberformel: Laufen, Lernen, Lieben, Lachen. Im Gegensatz dazu stehen die drei S: Sauber, Sitzen, Satt, die für ein Altern in Zufriedenheit und Würde nicht ausreichen. Die Angebote sollten also möglichst multifaktoriell und multisensorisch sein und viele unterschiedliche Impulse setzen. $\mathrm{Zu}$ diesen Forderungen können Themen aus den Bereichen Musik, Sprache und Bewegung/Tanz sehr viel beitragen, denn Musik ist ein besonderes, einzigartiges Medium. Es ist aus unser aller Leben nicht wegzudenken und spielt in sämtlichen Altersgruppen eine Rolle, wenn auch in unterschiedlichem Ausmaß, je nach Lebensgeschichte.

Der Einfluss der Musik beginnt schon in der pränatalen Phase und reicht bis zum Lebensende und damit bis in die Palliativmedizin sowie Sterbe- und Trauerbegleitung. John Miles bringt es in einem seiner Rocksongs mit folgendem poetischen Text auf den Punkt: „Music was my first love and it will be my last." (zitiert nach Wickel, 2013, S. 48). Da Musik ein affektnahes Medium ist, können durch sie Gefühle belebt werden, ohne Umwege über begriffliche Äußerungen, die im Alter manchmal schwerfallen. Musik kann in innerpsychische Schichten vordringen, die Worte nicht mehr erreichen, denn „ganz leicht und ohne Gewalt macht Musik die Türen der Seele auf“ (Sophie Scholl zitiert nach Hartogh \& Wickel, 2008, S. 26). Diese Eigenschaft der Musik ist besonders bedeutsam im Umgang mit dementiell erkrankten Menschen.

Es ist nicht erstaunlich, dass beim Hören, Machen, Verstehen und Erleben von Musik mehrere Regionen beider Gehirnhälften aktiviert werden. „Im Grunde genommen liegt man nicht falsch mit der Behauptung, dass Musik vom ganzen Gehirn gemacht und verarbeitet wird." (Spitzer, 2004, S. 208) Sie kann damit ein starker Reiz für neuronale Umstrukturierung sein, für die Verknüpfung neuer neuronaler Verbindungen. Dieser, in den Neurowissenschaften als Neuroplastizität bezeichnete Prozess, ist auf kein Alter beschränkt (vgl. Spitzer, 2004, S. 209). Auf die Zielgruppe der hochaltrigen Menschen bezogen, 
erklärt die folgende Aussage von Eckard Altenmüller, was wir in unseren musikalisch-tänzerischen Angeboten immer wieder beobachten können:

„Es ist so, dass Musik tatsächlich besonders tief eingeprägt zu sein scheint. Das mag daran liegen, dass sie sehr stark an Emotionen gebunden ist und dass $\mathrm{Mu}$ sizieren auf vielen verschiedenen Repräsentationen im Gehirn beruht. Eine Besonderheit bei Alzheimer zum Beispiel ist, dass der Schläfenlappen, in dem Hörverarbeitung im Wesentlichen vor sich geht, mit als Letztes von den Abbauprozessen betroffen ist." (Altenmüller zitiert nach Neumann, 2014, S. 34)

Immer wieder ergeben sich Situationen, in denen dementiell veränderte Personen, die zumeist wenig am Geschehen teilnehmen, plötzlich bei Liedern, die offensichtlich in ihrem Leben bedeutsam waren, mitsingen. Wie sinnvoll, weil sinngebend, ist es also, sich nicht erst im hohen Alter, sondern lebensbegleitend im aktiven Tun und Gestalten mit Musik, Sprache, Tanz und deren Verbindung auseinander zu setzen. Neben dieser sinnstiftenden Kraft der Musik erleben wir auch immer wieder ihre gemeinschaftsfördernde Wirkung. In der Gruppe ausgeführt bauen sich durch Musik Kontakt- und Kommunikationsbrücken auf. Sie bilden ein Gegengewicht zu sozialer Isolation, eines der Altersprobleme. In einem von Senior ${ }^{*}$ inn)en gerne gesungenen, österreichischen Lied von Anton Schmid heißt es so wahr: „Kömmt's lei eina in die Stubn, kömmts lei eina ins Haus, wann ma mitananda singen, gehn die Liadlan nit aus." (Drewes \& Mittergradnegger, 1975, S. 45)

\subsection{Beispiele künstlerisch-geragogischer Angebote in einem Seniorenheim}

Im Rahmen meiner Lehrtätigkeit am Carl-Orff-Institut der Universität Mozarteum $^{5}$, als Ausbildungsstätte von Musik- und Tanzpädagog( ${ }^{*}$ inn)en, konnte ich erleben, wie wirkungsvoll und belebend Musikangebote in der Altersgruppe betagter Menschen sind. Im Jahre 2003 startete ich mit einem wöchentlich stattfindenden Angebot in einem Seniorenheim, in das meine Mutter eingezogen war.

Dieses Angebot steht seitdem allen Bewohner( ${ }^{*}$ inne)n des Heimes offen, eben auch denjenigen, die glauben, unmusikalisch zu sein oder die sehr bewegungseingeschränkt sind. Auch dementiell veränderte Personen werden in-

5 Vielfältige Einblicke in die Thematik bietet folgende DVD der Autorin: Schönherr, C. \& Kallós, C. (2013): Ich bin wieder jung geworden, Künstlerisch-pädagogische Angebote für Menschen in hohem Alter, Universität Mozarteum Salzburg. DoppelDVD (deutsch/englisch) mit Booklet (http://shop.uni-mozarteum.at/de/ich-binwieder-jung-geworden.html [28.07.2020]). 
tegriert, wenn die Teilnahme für die Gruppe tragbar ist und von dem Pflegepersonal für richtig befunden wird. Dabei ist zu beobachten, wie sich noch rüstigere Teilnehmer*innen hilfreich bei denjenigen einbringen, die körperliche oder geistige Beeinträchtigungen haben.

Nachfolgend möchte ich beispielhaft Einblicke in unsere Stunden geben, wobei zwei Themenbereiche ausführlicher behandelt werden: das Aktive Musikhören sowie Bewegung/Tanz.

Unser musikalisch-tänzerisches Angebot im Seniorenheim basiert auf den grundlegenden Konzepten der Elementaren Musik- und Tanzpädagogik und deren Adaption an die Altersgruppe mit der Zielperspektive, dass die Musik in ihren Wirkweisen und deren Umsetzung in Bewegung erfahren, die Lebenswelt und musikalische Biografie der Teilnehmenden wertgeschätzt und weitergeschrieben, vorhandene Kompetenzen einbezogen, altersbedingte Schwierigkeiten und Beeinträchtigungen berücksichtigt, Grundbedürfnisse wie Selbstverwirklichung, Wertschätzung, Anerkennung und das Ausleben kreativer Fähigkeiten genährt und Freude und Gemeinschaft erlebt werden können. An dieser Stelle sei ein basaler Gedanke Carl Orffs genannt, der auch in der Arbeit mit Senior ${ }^{*}$ inn)en von entscheidender Bedeutung ist: „[...] sie [Elementare Musik] ist eine Musik, die man selbst tun muss, in die man nicht als Hörer, sondern als Mitspieler einbezogen ist.“ (Orff, 1963, S. 16). Durch handelnden Mitvollzug nähern wir uns den angeführten Zielperspektiven: Selbsttätigkeit wird angeregt, psychophysische und emotionale Bereiche werden belebt, das Selbstbewusstsein und die Selbstidentität erfahren und gestärkt. Zudem wird der Ausdrucksreduzierung sowie der sozialen Isolierung entgegengewirkt. Dies sind außermusikalische Transfereffekte der Musik, die sich sehr positiv auf die musikausübende Person bzw. auf die Gruppe auswirken.

\subsection{Aktives Musikhören im Rahmen der Senior( ${ }^{*}$ inn)engruppe}

„Mir bleibt der Atem weg, so schön ist es“ oder „Mir geht es durch und durch“. Diese Aussagen von Teilnehmer( ${ }^{\star}$ inne)n standen im Zusammenhang mit dem Marsch aus der Nussknacker Suite von Tschaikowsky, zu dem wir mit Pfauenfedern getanzt haben. Die tänzerische Leitidee dabei war: Zeige, was du hörst! ${ }^{6}$

In der Arbeit mit alten Menschen haben Musikpädagog( ${ }^{*}$ inn)en die kreative Aufgabe, dem gewählten Musikstück entsprechend Ideen zu entwickeln, welche die Gruppe vom Zuhören zum Mitagieren verführen, so dass alle am Musik- und damit am Ausdrucksgeschehen teilhaben können. Dabei sind dirigierende, tänzerische, instrumentale oder malende Aktivitäten unterstützend.

6 Vergleiche hierzu auch Pötschke, 1960. 
Musik-Erleben und Musizieren gehen auf diese Weise konform mit einem Leib-Erleben.

Das hat die Potenz, zu einem Musikhören zu führen, das Ergriffensein auslöst. In diese Richtung gehen die folgenden Worte einer Teilnehmerin: „Ich bin wieder jung geworden." Ihre Worte zeigen, dass Musik Erinnerungen wachrufen kann, die es ermöglichen mit Gefühlen und Erlebnissen wieder in Berührung zu kommen, die längst vergessen schienen. Die erinnerte Fähigkeit, in jüngeren Jahren mit Freude zu der in der Stunde gehörten Annenpolka getanzt zu haben, gehört nach wie vor zu dem Menschen, der heute aufgrund von Altersdefiziten im Rollstuhl sitzt.

Im Gegensatz zu meiner Arbeit mit Kindergruppen nimmt das Aktive Musikhören in den Stunden mit den Senior $\left({ }^{*}\right.$ inn)en einen weitaus größeren Raum ein. Dies hängt wohl damit zusammen, dass das Bewegen und Mitspielen zu eingespielter Musik mehr den Musikhörgewohnheiten der Teilnehmenden entspricht, als das selbst Musizieren und sich dazu zu bewegen.

\subsection{Zur Gestaltung von Bewegung und Tanz}

Die Tatsache, dass ein musikalisch-tänzerisches Angebot für hochbetagte Menschen im Wesentlichen ohne Fortbewegung im Raum auf einem Stuhle sitzend stattfindet, mag verunsichern. Ausgehend von Gruppen wie im Seniorenheim, bei denen Fortbewegung ohne Stock, Rollator oder Rollstuhl fast nicht möglich ist, kann die Frage aufkommen, ob es bei dieser Einschränkung überhaupt genügend Bewegungsmöglichkeiten gibt. Hierbei gilt es, neue Wertigkeiten $\mathrm{zu}$ entdecken und einen anderen Blick für den Körper zu entwickeln. Arme, Hände und Finger fungieren als Stellvertreter für den gesamten Körper. Verstärkt werden kann dies durch Einbeziehung von Objekten, wie z.B. Tücher, Federn, Stäbe, farbige Handschuhe oder Fingerpuppen. Sie regen zu freien Bewegungen an und helfen, Hemmungen abzubauen, wenn es darum geht, eigene Abfolgen ohne Vorgaben zu entwickeln. Dabei entstehen zum Erstaunen der Ausführenden manchmal Bewegungsmotive, die aufgrund von Beschwerden bereits aus ihrem Repertoire gestrichen wurden, aber nun dennoch möglich sind.

Wird der Fokus auf die Hände gelenkt, gilt es, sie in ihren tänzerisch-emotionalen Ausdrucksmöglichkeiten zu entdecken. Hierzu ein Beispiel aus der Praxis: Mit farbigen Handschuhen verkleiden wir unsere Hände. Diese Kostümierung regt die Fantasie an und stimuliert die Bewegung der Hände und Finger. Dabei kann es auch zu Handkontakten kommen, im Sinne zwischenleiblicher Berührung, die kontaktförderlich sind. Ohne den Schutz, den die Handschuhe bieten, würden sie kaum entstehen. 
Augen und Ohren erfreuen sich am farbenprächtig-elegant-fließenden Tanz der Hände, durch die z.B. melodische Abläufe in Camille Saint-Saëns Komposition Aquarium sichtbar und erlebbar werden. Stets ist eine achtsame Vorgehensweise, ein waches Beobachten von Seiten der Pädagogin oder des Pädagogen angesagt, damit die physische Leistungsgrenze der betagten Menschen nicht überschritten wird.

\subsection{Kriterien für die Musikauswahl}

Bei der Auswahl des Musikstückes sind die Länge, der Charakter, das Tempo und der Aufbau des ausgewählten Stücks zu bedenken. Dabei kann eine stimmliche und/oder instrumentale Live-Bewegungsbegleitung der leitenden Person von Vorteil sein. Im Gegensatz zu einem digital dargebotenen Musikstück kann sie sich an die Bewegungsmöglichkeiten der Teilnehmenden anpassen und Ideen musikalisch aufgreifen, unterstützen und vertiefen. Im Sinne einer multiplen Impulsgebung, die Kopf, Herz und Hand gleichzeitig anspricht und mit musikalisch-tänzerischen Angeboten für betagte Menschen verbunden ist, sollten wir uns auch bewusst sein, dass Hand und Finger ebenso wie Mund und Zunge besonders fein differenzierte Körperteile sind und ein großes Feld auf dem Motorischen Kortex, der sensomotorischen Hirnrindenregion einnehmen (vgl. Anzengruber, 2006, S. 32). Bewegungen mit Händen und Fingern aktivieren diese Region, was bei Pianisten sogar zu deren Vergrößerung führt, wie die Musiker-Plastizitätsforschung schon vor zwanzig Jahren nachweisen konnte (vgl. Altenmüller, 2018, S. 306). In dem Zusammenhang sei erwähnt, dass die Hände als das äußere Gehirn des Menschen bezeichnet werden, eine Aussage die Immanuel Kant zugeschrieben wird. Auch unsere Sprache weist in den Verben er-tasten, be-greifen, er-fassen auf diesen $\mathrm{Zu}$ sammenhang hin, der beim Kleinkind, das sich die Welt durch Betasten mit Fingern oder Lippen/Zunge aneignet, zu beobachten ist. Die Bewegungen der Arme und Hände verbunden mit Musik in kreativen Improvisationen und tänzerischen Gestaltungen sind aufgrund ihrer multisensorischen Impulsgebung einer reinen Armgymnastik sicherlich überlegen.

Das aktive Musikhören sowie Bewegung/Tanz sind dem Kernbereich der musikalisch-tänzerischen Aktivitäten zuzuordnen. Dazu gehört auch Body Percussion in Form von klatschen, patschen oder stampfen. Auf Schnipsen wird verzichtet, weil die Finger oft steif und unbeweglich sind. Im Hinblick auf die körperliche Situation der Teilnehmenden haben körpereigene Instrumente den Vorteil, dass sie den Kreislauf der Ausübenden anregen und durch Koordinationsimpulse das Koordinationsvermögen bspw. der Arme und Beine aktivieren. 


\subsection{Spiel auf Instrumenten und Singen}

Ein weiterer Themenkreis ist das Spiel auf Instrumenten, womit im Wesentlichen das sogenannte kleine Schlagwerk (Trommeln, Claves, Metallinstrumente, Rasseln oder Guiros) zum Einsatz kommt. Das klangfarbenreiche Orff-Instrumentarium bewährt sich wegen seiner guten Handhabung. „Die spielende Person kann sehen, fühlen und gleichzeitig hören, welche Art und Intensität der Bewegung zu welchem Klangereignis führt." (Regner, 1998, S. 97).

Gerne angenommen wird auch das Spiel auf Klangbausteinen, wohingegen bei Stabspielen die meisten Teilnehmenden eher zurückhaltend bis ablehnend reagieren, es sei denn, sie haben früher aufgrund ihrer Berufstätigkeit schon Kontakt mit diesen Instrumenten gehabt.

Das Singen hat in jeder Stunde ebenfalls seinen Platz, zumal der Gesang neben der Gemeinschaftsförderung als Königin der Atemübungen gilt und damit die Gesunderhaltung der Atemorgane unterstützt. Dieser salutogenetische Aspekt ist gerade in dieser Altersgruppe bedeutsam. Das Singen von Liedern stellt aber auch eine wichtige Erinnerungsbrücke in vorhergehende Lebensabschnitte dar und kann somit wiederum zur Identitätsstärkung beitragen.

\subsection{Sprache und Sprechen}

Der Themenkreis Sprache und Sprechen ist ein weiterer Bereich, der in der Altersgruppe betagter Menschen einen besonderen Stellenwert hat. Die Gruppenmitglieder können sich mit erlernten Gedichten oder Texten einbringen, oder sich z.B. durch einen wieder erinnerten Abzählvers in die Jugend zurückversetzen und mit Erstaunen erleben, wie daraus ein kleines Sprechstück (möglicherweise verbunden mit Rhythmusinstrumenten) werden kann.

An dieser Stelle möchte ich noch eine auch für die Musikgeragogik relevante Leitidee der Elementaren Musik- und Tanzpädagogik einbringen: Elementare Musik ist nie Musik allein, sie ist mit Bewegung, Tanz, Sprache verbunden. Durch das Wechselspiel der drei aufgeführten künstlerischen Bereiche sind die Angebote breitgefächert und sprechen auch Menschen an, die nicht gerne singen, weil sie vielleicht negative Erinnerungen aus der Schulzeit mit dem Singen verbinden. 


\subsection{Kreativ-schöpferische Freiräume}

In enger Verbindung mit den angeführten Themenkreisen steht die Aufbereitung der ausgewählten musikalischen Inhalte, bei der im Sinne der Elementaren Musik- und Tanzpädagogik die Bereiche Exploration sowie Improvisation und die daraus erwachsende Gestaltung einen wesentlichen Anteil ausmachen. Sie bieten Raum für das Finden, Entdecken und Ausleben spielerischer Bedürfnisse, denen auch in diesem Altersabschnitt begegnet werden soll. Improvisation belebt das in jedem Menschen innewohnende künstlerische Potenzial und regt zu aktivem Selbstausdruck an. Das Produzieren von Neuem steht im Gegensatz zu dem weit verbreiteten Altersleiden der körperlich-seelischen Rigidität. In Phasen des Explorierens, Experimentierens und Improvisierens zeigt sich eine Reaktivierung von Lebens-, Schaffens- und Spielfreude, die zur Stärkung des Selbstbewusstseins beiträgt, das durch die Konfrontation mit dem kognitiv-physischen Abbau zu schwinden droht. Gerade im motorischen Bereich ermöglichen Improvisationsaufgaben das Entdecken und Ausführen von Bewegungen, die an die jeweilig körperlichen Möglichkeiten angepasst sind. Das verhindert Überforderung und minimiert die frustrierende Erfahrung, das Geforderte nicht erfüllen zu können. Es ist notwendig, Bewegungen an die vorhandenen Fähigkeiten anzupassen. So schafft man Gelingensbedingungen, die den frustrierenden Erfahrungen des Alters entgegenstehen.

\section{Schlussgedanken}

Die Unterrichtsinhalte sollten bezüglich ihrer musikalischen Ansprüche so niedrigschwellig sein, dass Gelingen gewährleistet ist und sich musikalisches Erleben und Genießen einstellen können. Erlebnis hat Priorität vor Ergebnis und geht einher mit der Fähigkeit der Lehrperson, jede noch so einfache, musikalisch-tänzerische Keimzelle mit eben demselben künstlerischen Gespür darzubieten bzw. anzuleiten, wie es z. B. das komplexe Spiel eines klassischen Musikstückes erfordert. Um bei den Teilnehmenden wirklich anzukommen, ist eine Sprechweise nötig, die durch Zuwendung, Intention, verlangsamtes Sprechtempo und plastische Artikulation das Auffassungsvermögen der Teilnehmenden sowie eventuelle Hörbeeinträchtigungen berücksichtigt.

Immer wieder zeigt sich, dass Lernen auf emotionaler, motorischer sowie kognitiver Ebene auch in hohem Alter möglich ist. Der Kommentar einer Teilnehmerin „Da habe ich ja richtig wieder etwas dazu gelernt“ bestätigt dies. Offensichtlich tut es gut, im Alter Lernangebote zu erhalten und so die vorhandene Lernfähigkeit zeigen und sich selbst bestätigen zu können. 
Eine entscheidende Voraussetzung für das Gelingen einer Stunde, aus der alle froh und gestärkt herausgehen, ist der Aufbau einer gelösten, auf Empathie basierenden Atmosphäre, bestimmt durch Wertschätzung und Akzeptanz. Die daraus entstehende Beziehung ist „das Herzstück jeder pädagogischen Unternehmung" (Figdor \& Röbke, 2008, S. 83). Auch eine Portion Fröhlichkeit und respektvoller Humor sind wünschenswert, denn „Lachen und Lernen bilden ein Traumpaar" oder auch "Lachen vertreibt auf traumhafte Weise die Angst" wie es Gerald Hüther angesichts der angespannten Lage (gesundheitliche Bedrohung, Ausgangssperren und Social Distancing) aufgrund der im Frühjahr 2020 vorherrschenden Corona-Pandemie formuliert ${ }^{8}$. Ein Lächeln schlägt die Brücke vom Ich zum Du, unterstützt damit persönliche Beziehung. Die Gelotologie, die Wissenschaft der Auswirkungen des Lachens beschäftigt sich mit den körperlichen und psychischen Aspekten des Lachens. Herzhaftes Lachen belebt das Zwerchfell, den Atem- und damit Lebensmuskel und steht in enger Verbindung mit einem der angenehmsten und wohltuendsten menschlichen Gefühle, nämlich dem der Freude. Im Alter ist sie manchmal Mangelware. Durch unsere künstlerisch-geragogischen Angebote aus den Bereichen Musik, Sprache und Bewegung können wir ein Gegengewicht bieten, indem wir freudvolle Emotionen beleben.

\section{Literatur}

Altenmüller, E. (2018). Vom Neandertal in die Philharmonie. Berlin: Springer. https://doi.org/10.1007/978-3-8274-2186-9

Altenmüller, E. in einem Interview mit Neumann, B. (2014). Die Gänsehaut-Studie. Publik-Forum Extra Thema: Musik. Die Kunst, die zur Seele spricht (S. 34). Oberursel: Publik-Forum Verlagsgesellschaft mbH.

Anzengruber, C. (2006). "Ich bin wieder jung geworden" Musik und ihre psychophysische Wirkung auf Senioren. Diplomarbeit, Universität Salzburg.

Dahlke, R. (2018). Das Alter als Geschenk. München: Arkana.

Drewes, H. \& Mittergradnegger, G. (Hrsg.). (1975). Du, mei Hamat, ghearst mein. Lieder aus Kärnten - für Schule und Haus - für gleiche Stimmen. Klagenfurt: Johannes Heyn.

Figdor, H. \& Röbke, P. (2008). Das Musizieren und die Gefühle: Instrumentalpädagogik und Psychoanalyse im Dialog. Mainz: Schott.

Hartogh, T. (2008). Musikgeragogik- ein bildungstheoretischer Entwurf. Musikalische Altenbildung im Schnittfeld von Musikpädagogik und Geragogik. Augsburg: Wißner.

7 Titel eines Zertifikationslehrganges angeboten vom Bildungshaus Puchberg bei Wels, 30. Nov. - 2. Dez. 2012.

8 Professor Gerald Hüther in einer E-Mail an die Autorin. 
Hartogh, T. \& Wickel, H. H. (2008). Musizieren im Alter. Arbeitsfelder und Methoden. Mainz: Schott.

Metz, J. \& Pauls, R. (2004). Grundgedanken zur künstlerisch-musikalischen Arbeit mit Gruppen im späten Erwachsenenalter. In J. Ribke \& M. Dratsch (Hrsg.), Gestaltungsprozesse erfahren - lernen - lehren. Texte und Materialien zur elementaren Musikpädagogik (S. 108-112). Regensburg: Con Brio.

Orff, C. (1963). Das Schulwerk-Rückblick und Ausblick. In W. Thomas \& W. Götze (Hrsg.), Orff- Institut Jahrbuch 1963 (S. 16). Mainz: Schott.

Petzold, H. G. \& Berger, A. (1974). Integrative Bewegungstherapie und Bewegungserziehung in der Arbeit mit alten Menschen. In H. G. Petzold (Hrsg.), Psychotherapie mit alten Menschen (S. 398). Paderborn: Jungfermann.

Pötschke, M. (1960). Zeige, was du hörst. Frankfurt: Wilhelm Hansen.

Regner, H. (1998). Musik lieben lernen. Von der Bedeutung früher Begegnung mit Musik. Mainz: Schott.

Schönherr, C. \& Kallós, C. (2013). Ich bin wieder jung geworden, Künstlerisch-pädagogische Angebote für Menschen in hohem Alter. DVD, Universität Mozarteum Salzburg. Abrufbar unter: http://shop.uni-mozarteum.at/de/ich-bin-wiederjung-geworden.html [28.07.2020].

Spitzer, M. (2004). Musik im Kopf. Hören, Musizieren, Verstehen und Erleben im neuronalen Netzwerk. Stuttgart: Schattauer.

Wickel, H. H. (2013). Musik kennt kein Alter. Mit Musik alt werden: ein Mutmacher. Stuttgart: Carus. 

Marc Brand

\section{„Musik ist mein Leben“ \\ Gemeinsam Musiklernen im Seniorenalter - eine filmische Annäherung}

Schon in der Vergangenheit musizierten Senior( ${ }^{*}$ inn)en in Orchestern und Blasmusikvereinen oder sangen in Chören. Dies tut der überwiegende Teil von ihnen meist schon seit längerem. Neuerdings erlernen jedoch zunehmend mehr Menschen im Alter ein Instrument, fangen zu singen an oder nehmen nach einer langjährigen Unterbrechung das Musizieren wieder auf. Dies erfolgt häufig nicht im Einzelunterricht, an den man beim Neulernen eines Musikinstruments zunächst denken mag. Vielmehr lässt sich zunehmend ein $\mathrm{Mu}-$ siklernen in Gruppen und damit eine Gewichtung des sozialen Moments des Musizierens beobachten. Das Gruppenlernen ermöglicht Senior ${ }^{*}$ inn)en Kontakte, verbindet sie durch gemeinsames musikalisches Erleben, spornt viele $\mathrm{zu}$ Leistungen an und hat dadurch letztlich häufig positiven Einfluss auf ihr Wohlbefinden. Hermann Rauhe, einer der Pioniere in der Forschung zu Alter und Musik, verweist auf diese grundlegende Bedeutung des gemeinsamen $\mathrm{Mu}-$ sizierens im Alter:

„Insbesondere ältere Menschen tendieren dazu, die Aufmerksamkeit auf Symptome und Krankheiten zu richten, wenn sie allein sind. Dies ist weniger der Fall, wenn sie sich einer Gemeinschaft mit anderen Menschen anschliessen. Am besten, wenn sie zusammen singen und musizieren, denn das schafft Nähe und Geborgenheit, Zuversicht und Hoffnung." (Rauhe, o. J.)

Mit zu dieser Entwicklung des Musiklernens von Senior( ${ }^{*}$ inn)en trägt eine gesellschaftliche Neubewertung des Alters bei: Das lange Zeit vorherrschende Defizitmodell, das das Altern primär als Prozess zunehmender Einschränkungen sah, wird vom Leitbild der sich nach vorhandenen Ressourcen und Entwicklungschancen orientierenden Lebensgestaltung abgelöst. Heute wollen Senior $\left({ }^{*}\right.$ inn $)$ en aktiv sein. Nicht zuletzt durch die allen Bevölkerungsschichten zugänglichen Medienkanäle sind viele Menschen über die gesundheitliche Relevanz geistiger und körperlicher Aktivitäten informiert. Auch ist mittlerweile bekannt, dass Alters- und Krankheitsbilder das Verhalten von Menschen wesentlich beeinflussen können: So geht aus neueren Längsschnittstudien hervor, dass positive Altersbilder zu mehr Lebensqualität und gar zu einer höheren Lebenserwartung führen (vgl. Wurm, Diehl, Kornadt, Westerhof \& Wahl, 2017). Diese Ergebnisse spiegeln sich in den Aussagen von Senior $\left({ }^{*}\right.$ inn)en zur wohltuenden Wirkung des Musizierens auf Geist und Seele, verstanden als 
eine psychische Ausgleichsfunktion. Hinzu kommt, dass seit längerem in den meisten Berufen stetig Weiterbildungen gefordert werden und für habituell bildungsorientierte Menschen Lernen auch im Ruhestand selbstverständlich oder gar ein Bedürfnis ist. Ein Gestaltungselement des aktiven Alterns kann daher Musik als Bildung sein. Musiklernende Seniorinnen und Senioren sind dabei Vorbilder für andere, wie die Aussage von Rudolf aus der Musikgruppe Kaleidoskop ${ }^{1}$ zeigt:

„Vor ein paar Jahren lernte ich im Zug eine alte Dame kennen, sie war sechsundachtzig. Wir kamen ins Gespräch, und sie sagte, sie fahre jetzt nach Bern und lerne nochmals ein Instrument. Ich fragte sie, welches Instrument sie denn lerne, und sie sagte: Die Orgel! Dann dachte ich mir, also wenn ich fünfundsechzig bin, dann will ich nochmals ein neues Instrument lernen, und so bin ich dann auf den Kontrabass gekommen."

Viele ältere Menschen sind musikinteressiert, wissen aber nicht, dass heute eine Vielfalt an speziell auf sie ausgerichteten Musiklernangeboten besteht. Viele verspüren gar den Wunsch, ein Musikinstrument $\mathrm{zu}$ erlernen oder $\mathrm{zu}$ singen, trauen es sich aber nicht $\mathrm{zu}$, andere benötigen spezielle Informationen hierzu. Um solche Menschen anzusprechen, sind Filmportraits ideal, denn die Akteure können in der audiovisuellen Dokumentation selbst zu Wort kommen und in ihrem Umfeld sicht- und erlebbar werden. Musizierende Senior ${ }^{*}$ inn)en zu zeigen und ein interessiertes Publikum auf den besonderen Wert des Musiklernens und Musizierens im Alter aufmerksam zu machen, ist in Anbetracht bisheriger Forschungsergebnisse ${ }^{3}$ ein naheliegender

1 Die im Filmprojekt Musik ist mein Leben - Vier Portraits zum Musiklernen im Seniorenalter (Brand \& Camp, 2019) portraitierte Musikgruppe Kaleidoskop entstand im Rahmen des Programms für das Musiklernen im Alter der Stiftung proMusicante in Rapperswil, Kanton St. Gallen. Mittlerweile besteht die Musiklerngruppe infolge von Todesfällen und Heimeinweisungen nicht mehr in dieser $\mathrm{Zu}$ sammensetzung. Nähere Informationen zur Musikgruppe finden sich im Text weiter unten sowie im Begleittext des Filmprojekts Musik ist mein Leben.

2 Dieses und alle folgenden Zitate ohne weitere Quellenangabe stammen aus dem Filmprojekt: Brand, M. \& Camp, M.-A. (2019). Musik ist mein Leben - Vier Portraits zum Musiklernen im Seniorenalter.

3 In den vergangenen Jahren ist für den deutschsprachigen Raum eine beachtliche Anzahl an Fachbeiträgen zum Musiklernen im Seniorenalter und zur Musikgeragogik im Besonderen entstanden, die in der laufend aktualisierten Bibliografie der Deutschen Gesellschaft für Musikgeragogik abrufbar sind. Kehrer (2017) vermittelt Einblicke in den Klavierunterricht mit dementiell erkrankten Menschen, was die Frage nach Bildungsangeboten für Betroffene aufwirft. Im Forschungsbericht Musikalisch aktiv bis ins Alter der Hochschule Luzern - Musik (Brand, 2014) äußern sich Senior( ${ }^{*}$ inn)en und Musiklehrpersonen zum Musikunterricht im Alter. Eine stets neu inspirierende Quelle zum Themenkreis Musikgeragogik besteht in der Website des Kompetenzzentrums für Kulturelle Bildung im Alter und Inklusion - kubia, das als Herausgeberin der Publikationsreihe Kulturelle Bildung sowie der 
Schritt. ${ }^{4}$ Interessierte vermögen durch solche lebendigen Einblicke eine direkte Beziehung zu ihrer eigenen Lebenswelt herzustellen, weit mehr, als dies mittels Schrifttexten möglich wäre. Ein als Filmdokumentation mit Musikmitschnitten und Interviews angelegtes, an der Hochschule Luzern realisiertes Forschungsprojekt ermöglichte es, nicht nur weitere Daten zur Beantwortung von Fragen zum Musiklernen im Alter zu generieren, sondern auch einer breiten Öffentlichkeit Resultate zu vermitteln.

\section{Die Filmportraits}

Bei dem in den bisherigen Untersuchungen erhobenen Material zum Musiklernen im Alter erschien für viele der interviewten Senior ${ }^{*}$ inn $)$ en der soziale Aspekt besonders bedeutsam. Um diesem Aspekt in seinem Bedeutungsgehalt für die Musizierenden und weiteren Facetten nachzugehen, wurden drei Musikgruppen bei ihrem Musiklernen filmisch begleitet und auch interviewt. Das Ergebnis sind vier Kurzfilme - drei Gruppenportraits und ein Fokus Demenz - von je etwa sechs bis neun Minuten Dauer. Sie eignen sich zur Präsentation in verschiedenen Kontexten, etwa bei Veranstaltungen zur Vorbereitung auf den Ruhestand durch Organisationen für das Alter, bei Weiterbildungen von Lehrpersonen an Musikschulen, bei der Ausbildung von Lehrpersonen an Musikhochschulen, aber auch beim Informationsangebot auf Monitoren im öffentlichen Raum von Gemeinden, Bibliotheken, Alterseinrichtungen, Spitälern und Arztpraxen. ${ }^{5}$

Fachzeitschrift Kulturräume+ zeichnet. Eine Auswahl weiterer Beiträge zum Musiklernen und Musizieren im Alter findet sich in der Literatur am Ende des Beitrags.

4 Es gibt eine Reihe von filmischen Dokumentationen, die das Singen und Musizieren von älteren Menschen zeigen, so beispielsweise Young @ Heart (Regie: Stephen Walker, Fox Searchlight Pictures, UK 2008). Sie sind allerdings weniger aus einem Forschungsinteresse heraus entstanden.

5 Die Filmportraits sind verfügbar unter: https://www.hslu.ch/de-ch/musik/for schung/forschungsprojekte/musiklernen-und-musizieren-im-alter/ [29.07.2020]. 


\section{Die portraitierten Musikgruppen}

\section{Kaleidoskop, proMusicante Rapperswil ${ }^{6}$}

Leitung: Urs Krienbühl

Besetzung: 2 Violinen, Block-/Altflöte, Querflöte, Klarinette, Klavier, Kontrabass

Der Leiter des Ensembles, Urs Krienbühl, spielt nach Bedarf wahlweise auf verschiedenen Instrumenten mit.

Wie der Name des Ensembles andeutet, besteht Kaleidoskop aus einer bunt gemischten Gruppe. Bunt ist sie nicht nur aufgrund der breiten Palette an $\mathrm{Mu}$ sikinstrumenten, sondern auch wegen eines breiten Repertoires an unterschiedlichen Stilen wie Volksmusik und Folk, Latin, Evergreens, Salon- und Filmmusik. Und zwischendurch wird bei einem Stück auch mal mitgesungen. Die Gruppe Kaleidoskop weist ferner subtil auf eine bewusst gelebte Inklusion unterschiedlicher Lebenssituationen hin. So musizierte in diesem Ensemble bis zu seinem Tod ein an fortgeschrittener Demenz erkrankter Senior auf der Geige. ${ }^{7}$ Für diese Inklusionsleistung erhielt die Stiftung proMusicante von Alzheimer Schweiz, Sektion Luzern, den Fokuspreis 2017 für besondere Verdienste.

Silverhorns und Greenhorns, Raum Sissach - Basel ${ }^{8}$

Leitung: Thomas Heid

Besetzung: fünfzig bis sechzig Saxofone (Sopran-, Alt-, Tenor- und Baritonsaxofon) und Schlagzeug

Für das Abschlussprojekt seiner Ausbildung in Musikgeragogik an der Fachhochschule Münster (NRW) suchte der Basler Musikpädagoge Thomas Heid mittels eines Inserats Senior ( ${ }^{*}$ inn)en, die Saxofon im Gruppenunterricht erlernen wollten. Vorbedingungen wie etwa Notenlesen gab es dabei keine. Der Ansturm war überwältigend. Nahezu dreißig Lernwillige meldeten sich und bilden seither die Silverhorns, die bereits nach kurzer Lernzeit mit ihren Auftritten das Publikum zu begeistern vermochten. In einer zweiten Phase entstanden in gleicher Weise die Greenhorns. Durch den Einbezug eines körperbehinderten Mitspielers wird auch in dieser Gruppe Inklusion gelebt. Geprobt wird wöchentlich in fest bestehenden Sechsergruppen und einmal monatlich

6 Die Website der Stiftung proMusicante in Rapperswil ist verfügbar unter: https:// promusicante.ch/promusicante/aktuelles [29.07.2020].

7 In einem Fokus Demenz wurde eigens auf die Inklusion des dementiell erkrankten Seniors eingegangen. Verfügbar unter: https://www.hslu.ch/de-ch/musik/forschung/ forschungsprojekte/musiklernen-und-musizieren-im-alter/ [29.07.2020].

8 Die Website zu Aktivitäten der Saxofongruppen ist verfügbar unter: http://www. silverhorns.ch/Welcome.html [29.07.2020]. 
treffen sich alle zu Gesamtproben. Für seine Initiative durfte Thomas Heid im Jahr 2017 den Kulturpreis der Basler Kantonalbank entgegennehmen.

\section{Volksmusikgruppe Sarnen}

Coach': Oliver Holzenburg, Musikschule Sarnen

Besetzung: Konzertzither, Akkordzither, Mandoline, Gitarre, Cello

Die Musik der Sarner Landfrauen lebt von den eher feinen Klängen der Saiteninstrumente. Nach außen zum Ausdruck kommen die besonderen Schwingungen dieser Instrumente unter anderem bei gelegentlichen Auftritten in Alterseinrichtungen. Die dabei erzeugte Resonanz fließt zurück zu den $\mathrm{Mu}$ sikant(*inn)en, die mit ihrer Musik den betagten und an Demenz erkrankten Menschen Stunden der Freude und Lebensqualität geschenkt haben. Für einige Zeit und bis kurz vor ihrem Tod spielte in der Formation eine an Alzheimerdemenz erkrankte Seniorin mit. Für die Landfrauen ist das gemeinsame Spiel zugleich eine musikalische und soziale Aktivität. So setzen sie sich selbst immer wieder neue musikalische Ziele, weshalb sie ein Angebot der Musikschule Sarnen in Anspruch nehmen und sich von einer Instrumentallehrperson coachen lassen. Ebenso wichtig ist den Frauen das gemeinsame Zusammensitzen nach der Probe bei Kaffee und Kuchen, bei dem allerlei Neuigkeiten ausgetauscht werden und viel gelacht wird.

\section{Musik und Demenz}

Stellt das Musiklernen und Musizieren im Seniorenalter an sich bereits eine beachtliche Leistung dar, so ist eine solche bei der Inklusion von an Demenz erkrankten Menschen noch weit mehr gegeben. In der Seniorengruppe Kaleidoskop spielt seit etwa zwei Jahren der an fortgeschrittener Demenz erkrankte achtzigjährige Violinist Eber ${ }^{10}$ mit. Er erlernte das Geigenspiel in jungen Jahren und vorwiegend autodidaktisch. Als gebürtiger Brasilianer ist er sehr gut mit Latin-Musik vertraut und sein breites Musikrepertoire erstreckt sich weiter über Filmmusik, Evergreens, Jazz-Standards, Salonmusik, Klassik bis zu Weltmusik. Dass Eber die ihm bekannte Musik sehr leicht aus dem Gedächtnis abzurufen und zu spielen vermag, ist angesichts seiner schweren Erkrankung eine große Chance. Die in jungen Jahren vorwiegend über das Gehör angeeigneten Fähigkeiten erweisen sich beim Umgang mit seiner Demenzerkrankung als unschätzbares Kapital. Das sind wichtige Argumente, die für eine ge-

9 Die Volksmusikgruppe Sarnen trifft sich regelmässig zum gemeinsamen, selbst organisierten Musizieren, lässt sich jedoch in unregelmässigen Zeitabständen von einem an der Musikschule angestellten Musiklehrer coachen.

10 Die Altersangabe bezieht sich auf das Jahr 2020. 
zielte Förderung des Musiklernens von klein auf sprechen und die Bedeutung des Musizierens im Alter unterstreichen. Ebers Frau, die ihn jeweils zu den Proben begleitet, beschreibt die Musiziermöglichkeit in der Gruppe als großen Glücksfall für ihren Mann. In ihrem Interview brachte sie unmissverständlich zum Ausdruck, wie wichtig für ihn das Spielen in der Gruppe sei. Sie stellte fest, dass er nach dem Musizieren im Seniorenensemble ein anderer Mensch sei, führte dies auf die dabei bestehende normale Umgebung zurück und plädierte für eine verstärkte Inklusion dementer Menschen: „Wenn er in einer sogenannten normalen Umgebung ist, geht es viel besser. Deshalb finde ich, dass dies mehr gefördert werden sollte, was natürlich von der jeweiligen Gruppe akzeptiert werden muss."

Allerdings ist damit noch nicht geklärt, in welchem Verhältnis die Wirkungsanteile der Musik selbst und diejenigen, die durch die normale Umgebung entfaltet werden, stehen. Eine Inklusion dementer Menschen in normale Kontexte ist auf jeden Fall zu empfehlen, wenn dabei, wie bei Eber, vorhandene Ressourcen der Betreffenden angesprochen werden können. Musik und Tanz in entsprechenden Altersgruppen bieten jedenfalls idealen Raum für Menschen mit Demenz, weil Musik und Tanz auch in einem weit fortgeschrittenen Stadium der Erkrankung abrufbar sind und damit für die Betroffenen und ihr Umfeld Lebensqualität entsteht. Irene Bopp-Kistler, leitende Ärztin der Memory Clinic des Waidspitals in Zürich, sagt: „Ich bin überzeugt, dass diese Menschen noch eine extrem gute Lebensqualität haben können, wenn man sie emotional richtig abholt.“ (Pletscher, 2009) Dabei müssen stets individuelle Voraussetzungen beachtet und daran angepasste Settings erprobt werden. Maßstab für das Gelingen von Inklusion ist der Blick auf die Wirkung bei allen Beteiligten, denn auch in einem gut funktionierenden Setting ist ein situativ flexibles Reagieren auf momentane Befindlichkeiten gefragt, wie dies von einer Mitspielerin geschildert wird:

„Dann haben wir auch schon gemerkt, dass es ihm [Eber] nicht mehr so wohl war. Gerade, wenn wir an einem Musikstück üben mussten, wie es vor Kurzem geschah, dann war er etwas verloren. Dann haben wir einfach wieder etwas gespielt, das er gut kannte und das ihn wieder abholte. Das ist überhaupt nicht hinderlich oder störend.“

Seniorenensembles eignen sich besonders gut zur Inklusion von Menschen mit Demenz, da im Alter das Verständnis für Einschränkungen bei Menschen zunimmt und die gegenseitige Toleranz groß zu sein scheint. Dazu kommt, dass in vielen Musikgruppen mit älteren Menschen die rein musikästhetischen Aspekte nicht im Vordergrund stehen, sondern das gemeinsame Musikerleben und der soziale Zusammenhalt. So beschreibt der musikalische Leiter, Urs 
Krienbühl, die Reaktion der Mitspielenden auf seine Nachfrage zur Aufnahme Ebers in die Gruppe als sehr bereitwillig:

„Aufgrund von Ebers musikalischen Vorlieben bin ich zur Gruppe Kaleidoskop gelangt und habe sie gefragt, ob sie einverstanden wären, wenn er mal versuchsweise mitspielen würde. Und sofort waren alle restlos einverstanden und sagten, sie fänden es super, das sei doch genau das, was sie tun möchten: Integration $^{11}$, und dass möglichst viele Menschen den Zugang zu Musik finden würden." (Krienbühl)

Inklusion ist daher nicht eine Einweg-Disposition, sondern vielmehr ein Gewinn für alle, wie dies von Ebers Musikfreunden einhellig geschildert wird: „Eber ist eine große Bereicherung für uns alle!“

\section{Soziale Einbindung}

Soziale Kontakte sind insbesondere im Alter von weitreichender Bedeutung für das persönliche Wohlbefinden. Doch mit dem Älterwerden brechen gerade die wichtigen beruflichen Beziehungsnetze weg und private Beziehungen werden etwa durch Todesfälle weniger. Zuweilen empfinden Ruheständler die Beziehung zur Partnerin oder zum Partner als ein nahezu ausschließliches Aufeinanderbezogensein als eine Gefährdung: „Die Weiterbildung dient der Sicherung der Beziehung auch in der nachberuflichen Zweisamkeit. Sie ermöglicht Eigenständigkeit einerseits über neue soziale Kontakte, andererseits aber auch über die Beschäftigung mit einem Thema, was in reinen Freizeitangeboten weniger gegeben wäre." (Kulmus, 2013, S. 15-16). Bei kritischen Lebensereignissen bieten eigenständige soziale Kontakte eine ideale Möglichkeit, diese zu verarbeiten. Das eigene Musizieren wirkt sich positiv auf die Psyche aus und führt zusammen mit dem Gruppenerleben zu einer inneren Stärkung, wie ein Mitglied der Saxofongruppe zum Ausdruck bringt:

„Mein Mann und ich hatten auch eine etwas schwierige Zeit, weil wir einen Sohn verloren haben, und mein Mann hat in dieser Zeit begonnen zu singen und ich bin jetzt genau in dieser Zeit zum Saxofon gekommen, und uns beiden tut das einfach sehr gut! Es kommt so von innen heraus und es hilft einem einfach, mit solch schwierigen Situationen umzugehen."

Solche kritischen Lebensereignisse können auch andere, weniger schwerwiegende Trennungen sein, beispielsweise von Paarbeziehungen oder das Selbstständig-Werden der Kinder. Der Geist ist durch die Musik ausgerichtet auf

11 In dieser Aussage wird umgangssprachlich der Begriff Integration verwendet, tatsächlich gelebt wird jedoch Inklusion. 
Neues und von den damit verbundenen Neuorientierungen erhoffen sich die Betroffenen interessante Kontakte, die Erschließung neuer Lebensbereiche oder die (Wieder-)Aktivierung des eigenen kreativen Potenzials.

Musiklernen in der Gruppe bietet durch das gegenseitige Anspornen und Motivieren einen weiteren, wesentlichen Vorteil gegenüber dem Einzelunterricht. Das Gefühl der Zugehörigkeit bewirkt, dass der oder die Einzelne gegenüber den anderen Mitspielenden leistungsbezogen nicht zurückbleiben will, was sich in einem gewissen Leistungsbedürfnis ausdrückt. Da in Musikgruppen mit älteren Menschen in der Regel eine beträchtliche gegenseitige Toleranzspanne ${ }^{12}$ besteht, ist die Gefahr eines übermäßigen Leistungsdrucks klein und die intrinsische Leistungsbereitschaft ist durchaus positiv besetzt, wie dies aus der folgenden Äusserung einer Seniorin der Saxofongruppe hervorgeht:

„In der Gruppe will man ja mitmachen, also ist man auch motiviert, die Hausaufgaben zu machen, oder, man übt eben, dass es einem wohl ist in der Gruppe, und ich denke, das ist ein sehr positiver Anreiz, dass man über das Musizieren untereinander eine so gute Beziehung hat und sich freut, sich ein Mal pro Woche zu treffen, um gemeinsam Musik zu machen."

Das soziale Interesse durch die Anbindung an eine Gruppe kann sogar gegenüber dem rein musikalischen Interesse überwiegen. Generell erfüllt der soziale Aspekt in Musikgruppen von Seniorinnen und Senioren eine wichtige Funktion. Zuweilen dient die Gruppe als eine Art Familienersatz oder als Ergänzung. Es werden dabei Unternehmungen geplant und durchgeführt. Diese gemeinsamen Erlebnisse schmieden die Gruppe zusammen und die Teilnahme am Wohlergehen des oder der Einzelnen ist groß. Ein musikalisch aktiver Senior sagt:

„Wir sind im Prinzip alle auf derselben Welle und haben Spaß, zusammen zu spielen, und wenn einer krank ist, dann leiden wir alle mit, weil er einfach nicht da ist. Wir freuen uns auch, weil es eine Begegnung ist! Fünfundsechzig ist ein Schnitt, man kippt aus dem beruflichen Netzwerk raus und muss wieder irgendwas Neues suchen, und das ist natürlich super hier."

Solche und ähnliche Aussagen von Senior $\left({ }^{*}\right.$ inn)en decken sich weitgehend mit der Außensicht der musikalischen Leitungspersonen, die die soziale Dynamik auch für sich selbst als positiv beschreiben:

"Ich finde, das ist etwas vom Schönen, wenn ich dann in der Gruppe eigentlich nicht mehr die wichtigste Person im sozialen Bereich bin. Die Leute

12 Anders verhält es sich in altersdurchmischten Gruppen und wenn unter Teilnehmenden unterschiedliche musikästhetische Ansprüche bestehen sowie Gewichtungen bezüglich Leistung und Sozialerleben divergieren. Dabei können ernsthafte Spannungsfelder entstehen. Hinweise zu dieser Problematik in Chören finden sich bei Koch (2017). 
kommen und treffen sich, freuen sich aufeinander, haben sich etwas zu erzählen. Sie sind dann, wenn wir beginnen, sehr diszipliniert, das finde ich super, und das möchten wir ja auch. Und anschließend gehen sie noch auf die Promenade ins Restaurant, und die meisten von ihnen haben wirklich auch Freunde gefunden."

\section{Wirkungen des Musizierens auf Körper, Geist und Seele}

Mit dem aktiven Musizieren gehen starke Gefühle der Selbstwirksamkeit einher. Obwohl von den Musizierenden explizit so kaum erwähnt, scheint die musikalische Betätigung mit ihren geistigen Herausforderungen ein guter Ersatz für die mit dem Ruhestand wegfallenden beruflichen Aufgaben zu sein. Die Senior( ${ }^{*}$ inn)en können sich weiterhin beweisen, denn die musikalisch-technischen Prozesse bieten dazu inspirierende Herausforderungen. Dies kommt deutlich in der Aussage einer der Interviewten im Rahmen des Filmprojekts zum Ausdruck:

„Vom Musikalischen her ist es eher der Rhythmus, an dem ich arbeiten muss, und etwas ganz Neues, an dem ich noch recht zu arbeiten habe: die Improvisation, um aus sich herauszukommen und nicht alles nur so nach Noten richtig zu machen, sich an die Noten zu klammern. Das ist eine Herausforderung und aber auch befreiend! Es wird nie so ideal sein, aber es ist ein Probieren und das gibt mir ausreichend Genugtuung."

Musik beinhaltet eine ideale Verbindung von Geist, Körper und Seele, deren Aspekte beim Musiklernen und Musizieren alle in bestimmter Weise aktiviert werden. Die folgenden Äußerungen zweier Seniorinnen lassen tiefgreifende Wirkungen durch das aktive Musik-Erleben vermuten:

„Das gemeinsame Musikmachen ist halt schon was ganz anderes: also wenn du zusammensitzt, die Harmonie, das Ganze, das daraus entsteht, die Freude! Ich weiß noch, zu Beginn hatten wir so bestimmte Stücke, ich hätte tanzen können, als ich aus dem Haus ging, das hat mich so angeregt, das ist extrem, was das bringt."

„Musik ist halt schon etwas, das mich sehr tief anzusprechen vermag, Emotionen auslöst, mich berührt, wo ich einfach finde, das vermag eine Sprache so nicht, doch das Musizieren vermag das."

Die hier festgehaltenen Aussagen stehen für eine große Anzahl gleich oder ähnlich lautender Äußerungen von Senior $\left({ }^{\star}\right.$ inn)en, wie sie aus bisherigen Forschungsarbeiten bekannt sind (vgl. Hartogh, 2005; Mende, 1991; Muthesius, 2001; Smilde, 2009) und sie lassen damit eine deutliche Signatur positiver Wirkungen des Musizieren auf das Wohlbefinden des Einzelnen erkennen. 
Eine häufig mit dem Musizieren verbundene subtile Verarbeitung schwieriger Lebenssituationen mündet zuweilen in kreative Prozesse, aus denen Neues, wie etwa ein kleines Lied, entstehen kann. Obwohl die Seniorin im folgenden Beispiel ihr Lied als etwas ganz Einfaches, Schlichtes beschreibt, ist der damit verbundene subjektive Bedeutungsgehalt deutlich erkennbar:

„Ich habe in meinem Leben gemerkt, in schwierigen Situationen, wie das gut tut, wie es aber auch Emotionen auslösen kann, also Trauer und so. Das hat aber auch dazu geführt, dass ich einmal ganz zu Beginn mit der Gitarre ein ganz einfaches Liedchen gespielt habe und dann gemerkt habe, da muss noch ein Text hinzu, und das ist dann wirklich so ein ganz persönliches, ganz einfaches Liedchen geworden."

\section{Niedrige Eintrittsschwelle}

Wie die Erfolge von Thomas Heid mit den Silverhorns und Greenhorns eindrücklich beweisen, lässt sich ein komplexes Musikinstrument wie das Saxofon in der Gruppe neu erlernen. Bestimmt trug bei der Ausschreibung des Angebots der Hinweis, wonach zum Erlernen des Instruments keine musikalischen Vorkenntnisse vorausgesetzt werden, mit zum großen Interesse bei, denn viele lernwillige Senior ( ${ }^{*}$ inn)en befürchten vermeintliche Ansprüche, denen sie nicht genügen könnten und denen sie sich nicht (mehr) aussetzen wollen. Eine Seniorin, die ein paar Jahre vor ihrem Eintritt bei den Silverhorns einen Einzelunterricht besuchte, lässt durchblicken, dass die damalige Lehrperson wohl zu hohe Ansprüche an sie stellte und der Gruppenunterricht besser auf ihr Lernen abgestimmt sei. Und nicht selten sind es prägende negative Erfahrungen mit Musikunterricht im Kindesalter, die eine nachvollziehbare Scheu vor dem Musiklernen mit sich bringen, wie es eine Mitspielerin der Saxofongruppe sehr plastisch schildert:

„Mir hat man als Kind immer gesagt: Du bist nicht musikalisch, hör auf zu singen, du kannst das nicht! - Also, alle haben mir das Singen eigentlich stillgelegt, und ich habe einfach gewusst: Ich kann nicht singen! Und jetzt kommt Thomas und sagt: Doch, du kannst! Versuche es einfach und mache es! Und ich merke: es geht - und es geht immer besser!"

\section{Die eigene Musikbiografie weiterschreiben}

Die Aussage dieser Seniorin zu verletzenden Reaktionen des Umfeldes auf musikalische Äußerungen in der Kindheit weist auf diesbezüglich nicht selten gebrochene Biografien von Musikliebenden hin. Solche Verletzungen begleiten 
Menschen als Defizitgefühl oft über Jahre, bis sie sich im Alter zu einem Neuversuch entschließen. Gerade deshalb sind niederschwellige Angebote wichtig, denn diese Lernwilligen wollen sich nicht mehr der Gefahr verletzender Situationen aussetzen.

Ein wichtiger Faktor für erfolgreiche Musiklernangebote für Senior $\left({ }^{*}\right.$ inn)en ist daher, dass diese an unterschiedliche Musikbiografien anschließen können. Aus verschiedenen Gründen war es vielen Menschen in jungen Jahren nicht vergönnt, ein Instrument zu erlernen, vielleicht weil der Familie das Geld dazu fehlte oder weil bestimmte Angebote nicht bestanden. Zugleich nahm für sie aber über alle Lebensphasen das Musikhören einen wichtigen Platz ein. Andere wiederum haben den Musikunterricht über einen längeren Zeitraum besucht, mussten ihn dann aber aufgrund beruflicher Erfordernisse oder einer Familiengründung mit Kindern für längere Zeit unterbrechen. Auch ihre Musikbiografien können in Form von Konzertbesuchen und fleißigem Musikhören weiterleben und im Alter durch eigenes Musizieren wieder verstärkt aktiviert werden.

\section{Chancen für Musikschulen und Musiklehrpersonen}

Denken unerfahrene Instrumental- und Vokallehrpersonen beim Schlagwort Senior ( ${ }^{*}$ inn)enmusizieren zunächst an eingeschränkte instrumentaltechnische und musikästhetische Perspektiven, lösen sich Vorbehalte meist nach kurzer Zeit in der Unterrichtspraxis auf. Ressourcen des Lernens im Alter treten in den Vordergrund. Insbesondere junge Lehrpersonen äußern sich begeistert über das Senior(*inn)enmusizieren und rücken die speziellen Qualitäten des Lehrens in Musikgruppen mit älteren Menschen in den Vordergrund. So erhalten Lehrpersonen von den Senior ( ${ }^{*}$ inn)en direktes Feedback bezüglich der methodischen Arbeit, Lob und selbst persönliche Anteilnahme, wie sie von einer jungen Musikstudentin geschildert wird:

„Er [der Schüler] nimmt mich auf, so wie eine Tochter, und das ist auch für mich ein sehr schönes Gefühl. Es ist eine sehr freundschaftliche Ebene geworden. Er unterstützt mich in meinen Sachen, fragt immer nach, wie es mir geht, und er und seine Frau laden mich auch schon mal zum Essen ein [...] Im Unterricht nimmt er mich sehr ernst, und da bin ich seine Unterstützerin." (Brand, 2014, S. 27)

Eine das Senior $\left({ }^{*}\right.$ inn)enlernen kennzeichnende Qualität liegt demnach in der Kommunikation zwischen Erwachsenen, die im gegenseitigen Austausch von Wissen, Ideen und Erfahrungen sich beidseitig anregen und bereichern. Eine Lehrperson unterstreicht dies: „Dass du auf einer anderen Ebene kommunizie- 
ren kannst! Nicht dass es mit Kindern nicht schön ist, aber die Sachen haben eine andere Tiefe." (Brand, 2014, S. 26) Der Unterricht mit den durch Lebenserfahrung und Alter gestärkten Persönlichkeiten wird von Thomas Heid, dem Leiter der Saxofongruppen, als besonders attraktiv beschrieben:

„Was mich am meisten reizt, sind eigentlich die Charakterköpfe, die Persönlichkeiten, die mir da gegenüber stehen. Das ist sehr eindrücklich. Dann ist es ihre Begeisterung; sie haben sich freiwillig dafür entschieden und setzen sich auch dem Druck aus, zusammen in der Gruppe zu spielen. Also die Freude, die sie da entgegenbringen, und die Begeisterung ist das, was mir eigentlich am meisten gefällt."

Durch diese Aussagen zeigt sich, dass Musiklehrpersonen bezüglich des Unterrichts mit Senior $\left({ }^{*}\right.$ inn)en die allgemeinen Voraussetzungen der Erwachsenenbildung zu beachten haben. Dabei geht es nicht wie bei Kindern und Jugendlichen um Musikerziehung mit einer systematischen und womöglich methodenfokussierten Vorgehensweise, sondern vielmehr um eine Lernbegleitung, die sich primär an den individuellen Interessen und Bedürfnissen der Lernenden orientiert. Die Lehrperson muss dabei über eine hohe Kommunikationskompetenz verfügen, da sie als Coach nötige Impulse vermittelt und Lernangebote macht:

„Eben den Begriff Coach finde ich sehr gut dafür. Es gibt eigentlich nicht ein Ziel in dem Sinne, dass ich sage: Wir wollen jetzt erreichen, dass die doppelt so schnell spielen können oder laut und leise viel besser auseinanderhalten können. Also das ist eigentlich nicht das Ziel. Das Ziel von guter Musikpädagogik, denke ich, ist immer das Betrachten der Musik durch die Augen derer, die musizieren, und gucken: Was ist für die das Entscheidende, was wollen sie, was haben sie daran, was nützt ihnen, was bringt sie voran, und was weniger? Ob ich das Stück jetzt weniger toll finde oder ob das jetzt mein Lieblingsstil ist, das ist völlig unwichtig und Nebensache." (Holzenburg)

Eine solche Haltung der Lehrperson, wie sie von Oliver Holzenburg, dem Coach der Volksmusikgruppe Sarnen hier zum Ausdruck gebracht wird, bietet beste Voraussetzungen zu einem gelingenden Unterricht, spricht die Lernenden an und vermag eine positive Motivationsdynamik zu erzeugen. Die Senior $\left({ }^{*}\right.$ inn)en benötigen die Sicherheit, von ihrer Lehrperson in ihrem Lernen verstanden und geführt zu werden. Sie erleben und beschreiben solchen Unterricht als wohlwollend unterstützend, ermutigend und vertrauend. Für die Lehrperson werden die Früchte der gemeinsamen Arbeit dann wieder durch die Freude und Gelöstheit der Lernenden erlebbar, wie dies Urs Krienbühl, der Leiter der Gruppe Kaleidoskop zum Ausdruck bringt: 
„Bei den Kindern sieht man es, das Strahlen der Augen, es ist jedoch bei älteren Menschen genauso zu sehen, vor allem, so finde ich, wenn sie musizieren! Wenn sie sich lösen können, wenn der Druck wegfällt - so: Ich darf jetzt ja keinen Fehler machen! -, wenn ich das sehe, ist es total befriedigend.“

\section{Kompetenzzentrum Musikschule}

Für Musikschulen kann eine gezielte Strategie zum Musiklernen im Alter 50+ profilbildend wirken. Sie können mittels an die Zielgruppe angepasster Informationen auf ihre Angebote aufmerksam machen und sich diesbezüglich als Kompetenzzentren positionieren. An Musikschulen können dann interessierte und kompetente Musikschullehrkräfte eigene Fachgruppen gründen und es können Kooperationen mit Institutionen für Senior ( ${ }^{*}$ inn)en eingegangen werden.

Voraussetzung dafür ist die fachliche Weiterbildung von Lehrpersonen für den Unterricht im Senior( ${ }^{*}$ inn)enalter. Im Zentrum solcher Weiterbildungen stehen spezifische Aspekte des Erwachsenenlernens, das Wissen über altersbedingte Einschränkungen und entsprechende methodische Werkzeuge sowie Kommunikationskompetenzen. Gerade Letzteres erweist sich als wesentlicher Qualitätsschritt auch für den Unterricht mit Kindern und Jugendlichen, da dieser Schlüsselkompetenz der musikpädagogischen Praxis allgemein zu wenig Beachtung geschenkt wird. Empfehlenswert sind auch spezifische Weiterbildungen in Musik- und/oder allgemein Kulturgeragogik, die sich im engeren Sinne mit dem Musiklernen von Senior ( ${ }^{*}$ inn)en in Pflegesituationen befassen, wobei hier vertieft auf krankheitsbedingte Einschränkungen, wie etwa Demenz, eingegangen wird. Die Tätigkeiten von Musikgeragog( ${ }^{*}$ inn)en weisen auch starke Berührungsflächen zu Berufsprofilen der Sozialen Arbeit und der Pflegeberufe auf.

Das gemeinsame Musiklernen in der Gruppe erweist sich als ein vielschichtiges Betätigungsfeld nicht nur für die Senior( ${ }^{*}$ inn)en selbst, sondern auch für die sie anleitenden Musiklehrpersonen. Aus gesellschaftlicher Perspektive sind dabei die mit diesem Musiklernen verbundenen gesundheitsfördernden Aspekte wie starke Gefühle der Selbstwirksamkeit, die ausgleichende Wirkung auf Geist und Körper sowie die soziale Adhäsion und eine spezifische Eignung für Inklusion zu beachten. Öffentlichen und privaten Musiklernanbietern obliegt es, in der Zusammenarbeit mit Institutionen für das Alter vermehrt auf die Bildungsbedürfnisse von Senior( ${ }^{*}$ inn)en abgestimmte Musiklernangebote bereitzustellen und bekannt zu machen. 


\section{Literatur}

Brand, M. (2014). Musikalisch aktiv bis ins Alter - Eine Untersuchung zum Musiklernen bei älteren Menschen. Forschungsbericht der Hochschule Luzern - Musik 10. Verfügbar unter: https://zenodo.org/record/31344\#.W-RoR3pKjGI [29.07.2020].

Brand, M. \& Camp, M.-A. (2019). Musik ist mein Leben - Vier Portraits zum Musiklernen im Seniorenalter. Luzern: Hochschule Luzern - Musik. Verfügbar unter: https://www.hslu.ch/de-ch/musik/forschung/forschungsprojekte/musiklernenund-musizieren-im-alter/ [29.07.2020].

Hartogh, T. (2005). Musikgeragogik - ein bildungstheoretischer Entwurf. Augsburg: Wissner.

Kehrer, E.-M. (2017). Klavierunterricht mit dementiell erkrankten Menschen. Ein instrumentalgeragogisches Konzept für Anfänger. Münster: Waxmann.

Koch, K. (2017). Seniorenchorleitung. Empirische Studien zur Chorarbeit mit älteren Erwachsenen. Münster: LIT-Verlag.

kubia - Kompetenzzentrum für Kulturelle Bildung im Alter und Inklusion. Verfügbar unter: https://ibk-kubia.de/ [29.07.2020].

Kulmus, C. (2013). Warum im Alter noch lernen? Funktionen von Weiterbildung im Ruhestand. Education Permanente, 1, 15-16.

Mende, A. (1991). Musik und Alter. Ergebnisse zum Stellenwert von Musik im biographischen Lebensverlauf. Rundfunk und Fernsehen, 39 (3), 381-392.

Muthesius, D. (2001). "Schade um all die Stimmen..." - Erinnerungen an Musik im Alltagsleben. Wien: Böhlau.

Pletscher, M. (2009). Glück im Vergessen? Geschichten von Demenzerkrankten und ihren Betreuern [DVD]. Zürich: Schweizer Fernsehen SF Dok und Praesens Film AG.

Rauhe, H. (о. J.). Die Bedeutung des aktiven Musizierens im Alter [pdf]. Verfügbar unter: http://www.smpv.ch/myUploadData/files/ZV_DACH_2011_Rauhe.pdf [29.07.2020].

Smilde, R. (2009). Musicians as Lifelong Learners. Discovery through Biography. Delft: Eburon. https://doi.org/10.1007/978-3-531-91520-3_10

Wurm, S., Diehl, M., Kornadt, A. E., Westerhof, G. J. \& Wahl, H.-W. (2017). How do Views on Aging Affect Health Outcomes in Adulthood and Late Life? Explanations for an Established Connection. Developmental Review, 46, 27-43. https://doi.org/10.1016/j.dr.2017.08.002 


\section{Autorinnen und Autoren}

Stephanie Bangoura lebt und arbeitet als Ausbilderin für rituelle Tanzpädagogik mit Livemusik in Hamburg. Sie ist Gestalttherapeutin, Tänzerin und Rhythmikerin. Ihr Arbeitsschwerpunkt liegt in der Vermittlung integrativer und heilender Erfahrung im Tanz. 2011 absolvierte Bangoura einen Master in Performancekunst an der Universidade Federal da Bahia. 2016 gründete sie das Performancekollektiv Bangoura Group und leitet seither den Verein Tanz der Kulturen in Hamburg.

Persson Perry Baumgartinger, Dr., ist Independent Scholar, Dozent und Coach. Von 2017-2019 war er Senior Scientist am Programmbereich Zeitgenössische Kunst und Kulturproduktion (Kooperationsschwerpunkt Wissenschaft \& Kunst, Paris Lodron Universität Salzburg und Universität Mozarteum Salzburg). Seine aktuellen Forschungsschwerpunkte sind aktivistische Kunstund Kulturproduktion, angewandte Sprachwissenschaft, Sozialgeschichte, Trans/Gender Studies sowie Forschungsethik.

Marc Brand studierte Musik am damaligen Konservatorium Luzern mit Hauptfach Klarinette bei Antony Morf. Weitere Studien bei Peter Rieckhoff, Professor an der HDK Berlin, sowie bei Suzanne Stephens und Karlheinz Stockhausen folgten. Arbeitsbereiche am Institut Musikpädagogik der Hochschule Luzern liegen in Studienkoordination, Lehre und Weiterbildung. Themenschwerpunkte in Forschung und Entwicklung beinhalten das Musiklernen verschiedener Altersgruppen in ihren jeweiligen Lebenswelten, das Lernfeld Üben sowie Qualitätssicherung und -entwicklung an Musikschulen.

Regina Brandhuber promoviert und lehrt an der Hochschule für Musik Nürnberg. In ihrer Dissertation beschäftigt sie sich mit agilen Methoden aus der Softwareentwicklung und überträgt sie auf das musikalische Üben. Seit 2008 arbeitet sie an der städtischen Sing- und Musikschule Sulzbach-Rosenberg als Gesangslehrerin und Ensembleleiterin. Sie komponiert und veröffentlicht $\mathrm{Mu}-$ sicals und Kinderlieder zum Thema emotionale Eigenständigkeit. Integrative Unterrichtsformen bilden einen wesentlichen Bestandteil ihrer Arbeit.

Bettina Büttner-Krammer, Mag. ${ }^{a}$, ist Musikvermittlerin bei den Wiener Symphonikern. Davor baute sie als Konzertpädagogin das Musikvermittlungsprogramm Tonspiele des Tonkünstler-Orchesters Niederösterreich auf, arbeitete eine Zeit lang bei den Wiener Philharmonikern und entwickelte neue Kinder- 
und Jugendprojekte für das Mozarteumorchester Salzburg. Daneben führte sie Musikvermittlungsprojekte u. a. für das Festspielhaus St. Pölten, das Ernst Krenek Forum und die Jeunesse Musicale Österreich durch.

Julia Eibl ist Doktorandin an der Universität für Musik und darstellende Kunst Wien. Sie ist als Lern- und Musiktrainerin von Menschen mit Behinderung tätig. Schwerpunkt ihrer Tätigkeiten ist praktisch wie wissenschaftlich die Auseinandersetzung mit marginalisierten Gruppen. Davor absolvierte sie an der Universität für Musik und darstellende Kunst Wien ein instrumentalpädagogisches Studium im Fach Klavier sowie ein Studium der Bildungswissenschaft an der Universität Wien.

Erik Esterbauer, Ass. Prof. Dr., ist seit 2017 Assistenzprofessor im Fachbereich Musik und Tanz in Sozialer Arbeit und inklusiver Pädagogik (MTSI) am Department für Elementare Musik- und Tanzpädagogik am Orff-Institut der Universität Mozarteum Salzburg. Er studierte Psychologie, Komposition und Elementare Musik- und Bewegungspädagogik und arbeitet seit 20 Jahren im Bereich Kinder-, Jugend- und Familienpsychologie, Inklusion und Diversität.

Martina Fladerer absolvierte den Studiengang „Kultur und Wirtschaft“ mit den Fächern Germanistik/BWL an der Universität Mannheim, bevor sie an der Hochschule für Musik Nürnberg und an der Universität Mozarteum Salzburg ihre instrumentalpädagogischen Studien im Fach Klarinette aufnahm. Seit Oktober 2019 ist sie Stipendiatin des Doktoratskollegs „Die Künste und ihre Öffentlichkeit: Dynamiken des Wandels“ am Schwerpunkt Wissenschaft und Kunst, einer Kooperation der Universität Mozarteum Salzburg und der ParisLodron-Universität Salzburg und forscht zu partizipativen Musizierkonzepten für Erwachsene. Daneben ist sie in der Operndirektion des Salzburger Landestheaters und als freischaffende Musikvermittlerin tätig.

Stefan Greuter, Prof., ist Lehrender in den Bereichen Inklusion, Informatik und Musik und Leiter der Fachcommunity Inklusionspädagogik an der Pädagogischen Hochschule Tirol. Er studierte Lehramt für Hauptschulen mit den Fächern Mathematik, Musik, Informatik und Erziehungswissenschaft an der Universität Innsbruck und hat eine Ausbildung als Lerndesigner und Hochschuldidaktiker. Neben seiner Lehrtätigkeit an verschiedenen Haupt- und Mittelschulen und einer reformpädagogischen Schule war Greuter Assistent bei der Lebenshilfe Tirol (Betreutes Wohnen). Er ist als nebenberuflicher Musiker (Gitarre, Gesang) aktiv. 
Mona Heiler arbeitet als Projektmanagerin und Musikvermittlerin im $\mathrm{Zu}$ kunftslabor - einer Initiative der Deutschen Kammerphilharmonie Bremen. Sie studierte den deutsch-französischen Master Kulturvermittlung/Médiation culturelle de lart in Hildesheim und Marseille mit dem Schwerpunkt Musik. Während ihres Studiums fokussierte sie sich zudem auf kulturelle Bildung und Musik im öffentlichen Raum und entwickelte in diesem Rahmen das Projekt KlaWIR am Bahnhof. Neben der deutschlandweiten Durchführung des Projekts erforschte sie ebenfalls dessen gemeinschaftsbildendes Potenzial.

Beate Hennenberg, Ass. Prof. ${ }^{\text {in }}$ Dr. ${ }^{\text {in }}$, ist Assistenzprofessorin am Institut für Musikpädagogische Forschung, Musikdidaktik und Elementares Musizieren der Universität für Musik und darstellende Kunst Wien. Dort baute sie den Arbeitsbereich Inklusive Musikpädagogik auf. Ihre wissenschaftlichen Forschungsprojekte und Publikationen bewegen sich an der Schnittstelle zwischen musikhistorischen und -pädagogischen Kontexten von Heterogenität und Diversität und thematisieren gesellschaftliche Ausschlussmechanismen. Sie ist beteiligt am Erasmus+-Projekt Inclusive Pedagogy in Arts - Europe und Sprecherin der Senats-Arbeitsgruppe Barrierefrei.

Heike Henning, Univ.-Prof. ${ }^{\text {in }}$ Dr. ${ }^{\text {in }}$, ist Professorin für Instrumental- und Gesangspädagogik an der Universität Mozarteum Salzburg, Department für Musikpädagogik Innsbruck. Sie ist Leiterin des Zentrums für chorpädagogische Forschung und Praxis am Haus der Musik in Innsbruck. Sie lehrte an diversen deutschen Universitäten und Musikhochschulen Musik-, Instrumental- und Gesangspädagogik sowie Kinderchorleitung. Als Kinderchorleiterin und Chorpädagogin ist sie eine international gefragte Expertin. Ihre Forschungsschwerpunkte liegen im Bereich chorpädagogischer Praxis sowie in der instrumentalund vokaldidaktischen Entwicklungsforschung.

Mirjam Hoffmann, Prof. ${ }^{\text {in }}$ Dr. ${ }^{\text {in }}$ Diplom-Päd. ${ }^{\text {in }}$, ist Professorin für Inklusive Pädagogik an der Kirchlichen Pädagogischen Hochschule Edith Stein in Innsbruck/Stams. Sie promovierte an der TU Dortmund zum Thema Religiosität und psychische Gesundheit von Jugendlichen in Brasilien. Ihre besonderen Forschungsinteressen liegen neben Fragen der schulischen Inklusion in der inklusiven Erwachsenenbildung sowie der internationalen Kulturarbeit.

Julia Lutz, Univ.-Prof. ${ }^{\text {in }}$ Dr. ${ }^{\text {in }}$, ist als Professorin für Musikpädagogik und -didaktik mit Schwerpunkt Grundschule an der Folkwang Universität der Künste Essen tätig. Zudem lehrt sie am Institut für Musikpädagogik der Ludwig-Maximilians-Universität München (LMU). Sie wirkt an diversen interdisziplinären Projekten mit, darunter am Projekt Inklusionsdidaktische Lehrbausteine, im 
Rahmen dessen sie an der LMU die Basisqualifikation Musik + Inklusion entwickelt hat. Ihre Schwerpunkte in Lehre und Forschung sind neben der musikalischen Arbeit im Kontext von Heterogenität und Inklusion u. a. der Bereich Lebenslanges Lernen und die Vernetzung von Phasen in der Musiklehrer ${ }^{*}$ innen)bildung.

Anita Moser, Dr. ${ }^{\text {in }}$, ist Senior Scientist am Programmbereich Zeitgenössische Kunst und Kulturproduktion (Kooperationsschwerpunkt Wissenschaft \& Kunst, Paris Lodron Universität Salzburg und Universität Mozarteum Salzburg). Ihre Lehr- und Forschungsschwerpunkte liegen in den Bereichen Kulturmanagement, Freie Kulturarbeit, Gender Studies sowie Gegenwartskunst in der Migrationsgesellschaft.

Lisa Pfahl, Univ.-Prof. ${ }^{\text {in }}$ Dr. ${ }^{\text {in }}$, leitet den Lehr- und Forschungsbereich Disability Studies und Inklusive Bildung und die digitale Bibliothek bidok: behinderung - inklusion - dokumentation am Institut für Erziehungswissenschaften an der Universität Innsbruck. Sie ist stellvertretende Sprecherin der Forschungsplattform Center Interdisziplinäre Geschlechterforschung Innsbruck, des Forschungszentrums Medical Humanities und Mitbegründerin der Zeitschrift für Disability Studies (ZDS). Ihre Forschungsschwerpunkte sind Wissen, Bildung, Behinderung und Geschlecht sowie Fragen nach Subjektivierung in sozialen, kulturellen und ökonomischen Ungleicheitsverhältnissen.

Michelle Proyer, Ass. Prof.in Dr.in, ist Sonder- und Heilpädagogin und ist derzeit im Bereich der Inklusiven Pädagogik am Zentrum für Lehrer ${ }^{\star}$ innenbildung und dem Institut für Bildungswissenschaft der Universität Wien tätig. In Forschung und Lehre sind ihre Schwerpunkte die diversitätsbezogenen Intersektionen - v.a. in Bezug auf Kultur und Behinderung - in Schule und den darauf bezogenen Lebenswelten. Transdisziplinäre, -nationale und -kulturelle Ansätze durchziehen ihre Aktivitäten.

Renate Reitinger, Prof. in $^{\text {Dr. }}{ }^{\text {in }}$, ist Professorin für Musikpädagogik, Leiterin des Departments für Elementare Musikpädagogik/Musikpädagogik und Studienberaterin an der Hochschule für Musik Nürnberg. Sie war Vizepräsidentin für Studium und Lehre und Vorsitzende der Kommission für Gleichstellungsfragen an der Hochschule für Musik Nürnberg (2008-2017). Ihre Forschungsschwerpunkte liegen im Bereich der musikalischen Entwicklungsforschung mit Fokus auf Heterogenität und (früh-)kindlicher Musikalität. Aktuell führt sie Projekte zur Didaktik des Gruppenunterrichts und zu Musik/Kreativität mit wohnungslosen Jugendlichen durch. 
Bernhard Richarz, Dr. med., ist Facharzt für Psychosomatische Medizin, Psychiatrie, Psychotherapie und Psychoanalyse und Co-Leiter der Initiative tanzfähig in Berlin. Von klein auf lebt er mit einer Behinderung. Er organisiert Tanz, ist als Tänzer an verschiedenen Stücken beteiligt und unterrichtet seit 2007 Zeitgenössischen Tanz und vor allem Improvisation in körperlicher Vielfalt. Neben Beiträgen zur Initiative tanzfähig hat er u. a. zu NS-Psychiatrie und sog. Euthanasie, Disability Studies, nonverbalen Psychotherapieformen und Gruppenanalyse wissenschaftlich gearbeitet und veröffentlicht.

Virginie Roy unterrichtet zeitgenössischen Tanz an der Musik und Kunst Privatuniversität der Stadt Wien und doziert an der Sigmund Freud Privatuniversität Wien. Nach dem Tanzstudium am Conservatoire National Supérieur de Musique et de Danse in Lyon machte sie ihren Master in Klinischer Psychologie und den Abschluss in Kunsttherapie an der Universität Paris. Als Tänzerin/ Performerin wirkt sie an zahlreichen internationalen Projekten mit und unterrichtet als diplomierte Tanzpädagogin an renommierten Institutionen und Festivals.

Shirley Salmon lehrt seit 1984 am Orff-Institut der Universität Mozarteum Salzburg im Bereich Musik und Tanz in sozialer Arbeit und inklusiver Pädagogik, koordinierte diesen Studienschwerpunkt und leitete den entsprechenden Lehrgang. Sie studierte Musik (York University, England), absolvierte eine Ausbildung als Kindergarten- und Grundschullehrerin (Froebel Institut, London) und studierte Erziehungswissenschaften (Leopold-Franzens Universität, Innsbruck). Sie arbeitet seit über 40 Jahren mit Menschen mit unterschiedlichen Begabungen und Behinderungen. Sie hält Vorträge und Workshops im In- und Ausland und hat Bücher, eine DVD und zahlreiche Artikel veröffentlicht.

Nora Schnabl-Andritsch, ist Musikerin und Rhythmikerin. Sie unterrichtet Rhythmik an der Universität für Musik und darstellende Kunst Wien am Institut für Musik- und Bewegungspädagogik und lehrt an der Musik und Kunst Privatuniversität Wien im Studiengang Tanz. Ihre interdisziplinären und partizipativen Projekte liegen im Bereich der Musikvermittlung, der inklusiven Unterrichtskonzeption für heterogene Gruppen, der multisensorischen Wahrnehmungsbildung sowie der Improvisation und stellen freie künstlerische Gestaltungsprozesse in den Bereichen Musik-, Bewegungs- und Bildsprache in den Vordergrund. 
Christine Schönherr hat seit 2003 einen künstlerisch-pädagogischen Schwerpunkt im Bereich musikalisch-tänzerischer Angebote für Erwachsene, Senior $\left({ }^{*}\right.$ inn)en und hochbetagte Menschen. Neben ihrer langjährigen Lehrtätigkeit in zahlreichen Fachbereichen am Orff-Institut der Universität Mozarteum Salzburg ist sie zertifizierte Lehrkraft für AAP (Atemrhythmisch angepasste Phonation nach Coblenzer/Muhar) und diplomierte Yogalehrerin (BYO/ EYU). Sie ist Autorin zahlreicher Veröffentlichungen, u. a. einer Doppel-DVD (deutsch/englisch), und als Kursleiterin im In- und Ausland aktiv.

Michael Turinsky lebt und arbeitet als Choreograf, Performer und Theoretiker in Wien. Er studierte Philosophie an der Universität Wien. Als Performer kollaborierte er u. a. mit Bert Gstettner, Barbara Kraus und Doris Uhlich. Seine Kollaboration Ravemachine (mit Doris Uhlich) erhielt 2017 den Nestroy-Spezialpreis. Michael Turinsky hielt Vorträge und Workshops u. a. an den Universitäten Linz und Salzburg, an der College Art Association in New York sowie beim Impulstanz-Festival Wien und veröffentlichte in unterschiedlichen Fachzeitschriften und Journalen.

Evelyne Walser-Wohlfarter ist neben internationaler Lehrtätigkeit und Kunstprojekten mit dem Aufbau der Initiative tanzfähig, welche den zeitgenössischen Tanz um die Dimension der körperlichen Vielfalt erweitern möchte, in Österreich beschäftigt. Sie studierte Elementare Musik- und Tanzpädagogik mit den beiden Schwerpunkten Musik und Tanz in Sozialer Arbeit und Inklusiver Pädagogik und Tanz am Carl-Orff-Institut der Universität Mozarteum Salzburg und Choreographie am Dartington College of Arts (GB).

Hana Zanin-Pauknerová ist freischaffende Choreografin, Pädagogin sowie Tänzerin und auf zeitgenössischen inklusiven Tanz und Tanztheater spezialisiert. Sie ist Obfrau des Kultur- und Bildungsvereins Ich bin O.K., künstlerische Leiterin des Ich bin O.K.-Tanzstudios und die Mitgründerin der Ich bin O.K.-Dance-Company in Wien. Ihre Ausbildung in den Bereichen Tanz und Pädagogik erhielt sie am Prager Tanzkonservatorium und an der Folkwang Universität der Künste in Essen. 